\title{
Environmental Degradation of Carbon Fibre-Reinforced Epoxy Composites: An Experimental Investigation
}

\author{
by \\ Jeffrey S Carette \\ A thesis submitted to \\ the Faculty of Graduate and Postdoctoral Affairs \\ in partial fulfilment of \\ the requirements for the degree of \\ Master of Applied Science \\ in \\ Materials Engineering
}

Ottawa-Carleton Institute for Mechanical and Aerospace Engineering

Department of Mechanical and Aerospace Engineering

Carleton University

Ottawa, Ontario, Canada

August 2017

Copyright (C)

2017 - Jeffrey S Carette 
The undersigned recommend to

the Faculty of Graduate and Postdoctoral Affairs

acceptance of the thesis

\title{
Environmental Degradation of Carbon Fibre-Reinforced Epoxy Composites: An Experimental Investigation
}

\author{
Submitted by Jeffrey S Carette \\ in partial fulfilment of the requirements for the degree of \\ Master of Applied Science
}

Dr. Jeremy Laliberté, Supervisor

Dr. Michele Faragalli, Co-Supervisor

Dr. Ronald Miller, Department Chair

Carleton University

2017 


\section{Abstract}

Work presented herein investigates the chemically-induced degradation of a carbonfibre reinforced epoxy. Monolithic coupons were conditioned from either single-sided exposure to, or complete immersion in one of five fluids; turbine oil, hydraulic fluid, ethylene glycol, deionized water or ocean water. At predefined intervals, coupons were removed from conditioning; subjected to evaluation of mass, dimensions and hardness; and destructively tested, analyzing either flexural properties or short beam strength. Secondary testing, including microscopy, x-ray micro-computed tomography and thermal analysis, was performed to further characterize degradation.

It was demonstrated that significant mechanical and physical degradation resulted from exposure to deionized and ocean water, but not from exposure to hydraulic fluid, turbine oil, or ethylene glycol. Water uptake, as evaluated by sample mass change, was non-Fickian and caused a measurable loss of constituent material. This was predicted to be due to water's polarity, allowing it to disrupt both epoxy's intermolecular and intramolecular bond structure. 
To the loving memory of my grandfather, Donald J. Langdon who, through his insatiable curiosity, taught me to never stop learning. 


\section{Acknowledgments}

I would like to acknowledge and thank the following individuals and associations for their contributions to my research efforts, without which, this project would not have been possible.

- To my supervisor, Jeremy Laliberté for his positivity and support throughout this project, provided through his breadth of engineering knowledge, insightful review of my work and ability to encourage self-driven conceptualization through a multitude of suggestions.

- To my co-supervisor, Michele Faragalli and mechanical engineer Lynne Kincaid for their industry-based guidance and prudent review of my work.

- To the Natural Sciences and Engineering Research Council of Canada for supporting this research through the contribution of a Collaborative Research and Development Grant.

- To General Dynamics Missions Systems - Canada for their in-kind contributions to the project.

- To machine shop technologists, Ian Lloy, Alex Proctor and Kevin Sangster for their amiability, solid judgment and guidance in the machine shop. 
- To laboratory technologists, Stephan Biljan, David Raude and Steve Truttmann for their in-laboratory training and willingness to offer insight and assistance whenever possible.

- To undergraduate researcher, Courtney L'Arrivée for her assistance with early-stage sample manufacturing, conditioning and testing processes.

- To the gentlemen of MC 3041 who's humour and intriguing perspectives helped preserve my mental health while on our unified journey through graduate studies.

- To my parents, Peter Carette and Suzanne Langdon for their continuous support of, and interest in, my educational pursuits.

- To my girlfriend, Julia Vit for her companionship, support and for being my partner on the bridge between school and life outside academia. 


\section{Contributions}

To General Dynamics Missions Systems - Canada (the industrial partner), this thesis has contributed knowledge of the effects of exposure to various fluids - common to aerospace - on the mechanical properties of carbon fibre-reinforced epoxy. To the composite community, this work has provided a greater understanding of the fluidinduced degradation mechanisms of carbon fibre-reinforced epoxy, validation of prior research observing the effects of its exposure to deionized and ocean water and presented a novel approach to evaluating rates of fluid ingress and residual properties resulting from single-sided exposure.

Components of this work have contributed to the conference paper "Mechanical Properties of Chemically Degraded Aerospace Fibre-Reinforced Polymer Matrix Composites" which has been submitted for publication in the CANCOM 2017 Proceedings of the 10th Canadian-International Conference on Composites. This paper was coauthored by Jeremy Laliberté of Carleton University and Lynne Kincaid and Michele Faragalli of General Dynamics Missions Systems - Canada, all of whom advised the work performed but were not directly responsible for the paper's contents. It is anticipated that components of this work will be included as preliminary elements of future publications investigating x-ray micro-computed tomography as a means of evaluating composite degradation and the synergistic contributions of water ingress and low-velocity impact on composite residual strength. 


\section{Table of Contents}

Acknowledgments $\quad$ v

List of Tables $\quad$ xii

List of Figures $\quad$ xiv

Nomenclature $\quad$ xix

1 Introduction 1

1.1 Motivation . . . . . . . . . . . . . . . . . 1

1.2 Organization of Thesis . . . . . . . . . . . . . . . 3

1.3 Background .......................... 3

1.3.1 Advanced Composite Design . . . . . . . . . . . . . 4

1.3.2 Composite Certification ............... 5

1.3.3 Design of Experiment . . . . . . . . . . . . . 10

1.4 Statistical Analysis . . . . . . . . . . . . . . . . 13

1.4.1 Hypothesis Testing . . . . . . . . . . . . . 15

1.4 .2 Analysis of Variance . . . . . . . . . . . . . . 16

1.4.3 Tukey's test and Dunnett's Test . . . . . . . . . . . . . . 19

1.5 Scope of Research . . . . . . . . . . . . . . . . . . . . 21

1.6 Literature Review . . . . . . . . . . . . . . . . . . . . . . . . . . . 22 
1.6.1 Review Taxonomy . . . . . . . . . . . . . . . . . . 22

1.6.2 Review Structure . . . . . . . . . . . . . . . . 23

1.6.3 Environmental Factors . . . . . . . . . . . . . 25

1.6.4 Evaluation Techniques .................. 44

1.6.5 Review Summary . . . . . . . . . . . . . 51

2 Methodology $\quad 54$

2.1 Experiment Proposal . . . . . . . . . . . . . . . . 54

2.2 Flexural Testing . . . . . . . . . . . . . . . . 57

2.2.1 SBS Testing . . . . . . . . . . . . . . 60

2.2.2 Fixture Fabrication . . . . . . . . . . . . . . . . . 61

2.2.3 Fixture Validation ... . . . . . . . . . . . . . 63

2.3 Coupon Manufacturing . . . . . . . . . . . . . . 66

2.4 Sample Conditioning . . . . . . . . . . . . . . . . . . . 69

2.4.1 Single-Sided Conditioning Fixture . . . . . . . . . . . . . . . 69

2.4.2 SBS Conditioning . . . . . . . . . . . . 78

2.5 Moisture Uptake . . . . . . . . . . . . . . . . . . 80

2.5.1 Metrology ........................ 85

2.6 Supplementary Evaluation . . . . . . . . . . . . . . 86

$\begin{array}{llr}3 & \text { Results } & 87\end{array}$

3.1 Campaign One: Single-Sided Flexural . . . . . . . . . . . . . . . 88

3.1.1 Moisture Absorption . . . . . . . . . . . . . . . . 88

3.1.2 Sample Metrology . . . . . . . . . . . . . . . . . . . . . . . . 92

3.1.3 Flexural Properties . . . . . . . . . . . . . . . . . . 93

3.1.4 Chemical Considerations . . . . . . . . . . . . . . . 104 
3.1.5 Surface Microscopy . . . . . . . . . . . . . . . . . . . . . . 109

3.1.6 X-Ray Micro-computed Tomography . . . . . . . . . . . . . 112

3.2 Campaign Two: Immersed SBS . . . . . . . . . . . . . . . 117

3.2.1 Moisture Absorption . . . . . . . . . . . . . . . . 117

3.2 .2 Sample Metrology . . . . . . . . . . . . . . . . . . . . . . . 129

3.2 .3 Short Beam Strength . . . . . . . . . . . . . . . . 135

3.2.4 Hardness Evaluation . . . . . . . . . . . . . . . . . 140

3.2.5 Thermal Analysis . . . . . . . . . . . . . . . . . 145

4 Experiment Evaluation $\quad 151$

4.1 Temperature Variance . . . . . . . . . . . . . . . . . . . . . . 151

4.2 Fluid Evaporation . . . . . . . . . . . . . . . . . . 156

4.3 Gasket Moisture Uptake . . . . . . . . . . . . . . . . . . . . . . . . 159

$\begin{array}{lll}5 & \text { Conclusions } & 161\end{array}$

6 Recommendations 164

6.1 Alterations to Single-Sided Exposure Conditioning Fixture . . . . . . 164

6.2 Extended Single-Sided Exposure . . . . . . . . . . . . . . . 165

6.3 Chemical Analysis . . . . . . . . . . . . . . . 165

6.4 Alternative Chemical Exposure . . . . . . . . . . . . . 166

6.5 Cyclic Exposure . . . . . . . . . . . . . . . . . . . . 166

6.6 Tomographic Analysis of SBS Samples _ . . . . . . . . . . . . 167

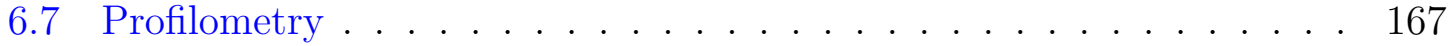

6.8 Synergistic Effects of Impact . . . . . . . . . . . . . . . 167

$\begin{array}{ll}\text { References } & 168\end{array}$ 
Appendix A Extended Background and Literature Review

A.1 Supplementary Background . . . . . . . . . . . . . . . . . . 181

A.2 Supplementary Literature Review . . . . . . . . . . . . . . . 184

A.2.1 Ultraviolet Radiation . . . . . . . . . . . . . . . . . . 184

A.2.2 Biological . . . . . . . . . . . . . . . . . 187

A.2.3 Salt Fog . . . . . . . . . . . . . . . . . . . . . . . . . 189

A.2.4 Galvanic Reaction . . . . . . . . . . . . . . 190

$\begin{array}{lll}\text { Appendix B Standard Operating Procedures } & 192\end{array}$

B.1 Single-Sided Flexural Sample Fluid Uptake Metrology: Standard Operating Procedure . . . . . . . . . . . . . . . . . 193

B.1.1 Preface. . . . . . . . . . . . . . . . . . . 193

B.1.2 Procedure . . . . . . . . . . . . . . . . . . . . . . . . 193

B.2 Short Beam Shear Fluid Uptake Metrology: Standard Operating Pro-

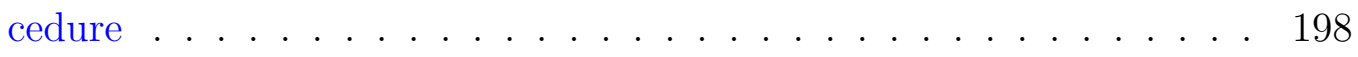

B.2.1 Preface. . . . . . . . . . . . . . . . . . . . . 198

B.2.2 Procedure . . . . . . . . . . . . . . . . . . . . . . . . 198

B.3 25 kN MTS 810 Test Frame Flexural Testing: Standard Operating Procedure . . . . . . . . . . . . . . . . . . . 201

B.3.1 Preface. . . . . . . . . . . . . . . . . . . . . 201

B.3.2 Procedure . . . . . . . . . . . . . . . . . . . . . 201

$\begin{array}{ll}\text { Appendix C Orthographic Drawings } & 213\end{array}$

$\begin{array}{ll}\text { Appendix D Supplementary Results } & 223\end{array}$

$\begin{array}{lll}\text { Appendix E } \text { Micro-computed Tomography Code } & 234\end{array}$ 


\section{List of Tables}

1.1 Potentially testable factor-level combinations. . . . . . . . . . . . 13

1.2 Effects and associated DoF for two-way ANOVA. . . . . . . . . . . 19

1.3 Potential sources of degradation, their probability of occurrence and severity of damage. . . . . . . . . . . . . . . . . 27

2.1 Offset yield strength and moduli values averaged over each 6061-T6, T561 test group. . . . . . . . . . . . . . . . . 64

2.2 Chemical composition of AX-5180 prepreg, adapted from [1] . . . . . 66

3.1 Specified and utilized test parameters for ASTM D5229, Procedure D; deviations from the specified standard highlighted in bold. . . . . . . 88

3.2 Specified and utilized test parameters for ASTM D5229, Procedure $B$; deviations from the specified standard highlighted in bold. . . . . . . 89

3.3 Standard deviation of percentage mass change, expressed in terms of duration and fluid. . . . . . . . . . . . . . . . 90

3.4 Rates of change of percentage moisture absorption. . . . . . . . . . . 92

3.5 Two-factor ANOVA summary for percentage change of sample length, width and thickness; significant results are highlighted in bold. . . . 94

3.6 Specified and utilized test parameters for ASTM D7264; deviations from the specified standard highlighted in bold. . . . . . . . . . . 95

3.7 Flexural and metrological data for baseline flexural samples. . . . . . 98

3.8 Two-factor ANOVA summary for change in flexural strength and modulus. . . . . . . . . . . . . . . . . . 100

3.9 Summary of Dunnett and Tukey post hoc statistical analysis of flexural data; significant differences in mean values are highlighted in bold. . 103 
3.10 Parameters used to approximate Fickian diffusion profiles. . . . . . . 125

3.11 Saturation times by conditioning fluid. . . . . . . . . . . . . 126

3.12 Two-factor ANOVA summary for change in SBS sample length, width and thickness; significant results highlighted in bold. . . . . . . . . . 131

3.13 Specified and utilized test parameters for ASTM D2344; deviations from standard are highlighted in bold. . . . . . . . . . . . . . . 136

3.14 Specified and utilized test parameters for ASTM D2240; deviations from standard highlighted in bold. . . . . . . . . . . . . . . . . 141

4.1 Array temperature distribution over conditioning durations. . . . . . 153

4.2 Flexural sample groups exposed to water conditioning, comparing dry-time, mass change and residual strength; standard deviations of varying-dry-time groups highlighted in bold. . . . . . . . . . . . . . . 158

A.1 Cooper's taxonomy of literature reviews with characteristics pertinent to the study highlighted in bold [2]. . . . . . . . . . . . . . . . . . . 182

A.2 List of extracted codebook categories. . . . . . . . . . . . . . . . 183

D.1 SBS sample groups exposed to water conditioning, comparing dry-time, mass change and residual strength. . . . . . . . . . . . . . 226

D.2 Two-factor ANOVA summary for SBS testing. . . . . . . . . . . 228

D.3 Summary of Dunnett and Tukey post hoc statistical analysis of SBS data; significant differences in means highlighted in bold. . . . . . . . 228 


\section{List of Figures}

1.1 Examples of I) unidirectional II) woven and III) short fibre, fibrereinforced composites. . . . . . . . . . . . . . . .

1.2 The hierarchy of the building block approach used to incrementally assess aircraft components, adapted from [3]. . . . . . . . . . 7

1.3 Geometric illustration of factorial design. . . . . . . . . . . . . . . . 12

1.4 Kinetic scheme for hydrocarbon oxidation, adapted from [4]. . . . . . 29

1.5 Change in composite mass as a function of time for epoxy-glass composite samples exposed to $30{ }^{\circ} \mathrm{C}(\star), 50^{\circ} \mathrm{C}(\triangle), 70^{\circ} \mathrm{C}(\diamond)$, and $90^{\circ} \mathrm{C}$ ( $\square)$ hydrothermal ageing, adapted from $[5] \ldots . . . . . . . .$.

1.6 Non-Fickian mass-uptake behaviours over long duration exposure represented in terms of the square-root of non-dimensional time, adapted

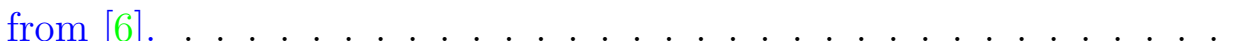

2.1 Flexural fixture assembly in a SBS configuration. . . . . . . . . . . 62

2.2 Stress-strain relationship for 6061-T6, T651 aluminum flexural test. . 64

2.3 Cure cycle used for flexural coupon parent laminate. . . . . . . . . . . 67

2.4 Cure cycle used for SBS coupon parent laminate. . . . . . . . . . 68

2.5 Image of representative flexural (AA15) and SBS sample (F6). . . . 69

2.6 Cross-section of proposed conditioning fixture illustrating i) top-plate, ii) gasket, iii) sample and iv) bottom-plate. . . . . . . . . . . . . . 71

2.7 Preliminary design of 18 -well conditioning array. . . . . . . . . . . . . 71

2.8 Small-scale test array with clamps placed to prevent cell crosscontamination. . . . . . . . . . . . . . . . . . 74

2.9 Full-scale array assembly. . . . . . . . . . . . . . . . . . . 75 
2.10 Assembled array structure. . . . . . . . . . . . . . 76

2.11 Array during fluid retention testing with polyimide tape applied over select wells. . . . . . . . . . . . . . . . . . . . 77

2.12 Schematic of finalized oven configuration. . . . . . . . . . . . . 79

2.13 SBS samples immersed in fluids, positioned in test tube rack. . . . . 80

3.1 Change if mass of flexural samples as a function of $\sqrt{\text { hours }}$ of exposure. 90

3.2 Two flexural samples illustrating a tool-side with parallel fibres (top) and bag-side with perpendicular fibres (bottom). . . . . . . 96

3.3 Stress-strain curves of unconditioned samples; failure of samples with top-surface fibres parallel and perpendicular to sample length. . . . . 97

3.4 Normalized flexural strengths as a function of conditioning liquid and duration. . . . . . . . . . . . . . . . . . .

3.5 Normalized flexural moduli as a function of conditioning liquid and duration. . . . . . . . . . . . . . . . . . . .

3.6 Normalized flexural strength as a function of percentage change in mass resulting from fluid uptake. . . . . . . . . . . . . . . . . . . 104

3.7 Molecular structures of i) cyanoguanidine, ii) poly[(phenyl glycidyl ether)-co-formaldehyde], iii) poly(bisphenol A-co-epichlorohydrin), glycidyl end-capped and iv) fumed silica. . . . . . . . . . . . . . 105

3.8 Molecular structure of a crosslinked DGEBA thermoset polymer, adapted from $[7] \ldots \ldots \ldots \ldots \ldots$

3.9 Molecular structures of i) water, ii) ethylene glycol and iii) 1-decene. . 107

3.10 Surface structures of samples exposed to i) air (control), ii) hydraulic fluid, iii) turbine oil, iv) ethylene glycol, v) ocean water and vi) deionized water after a three-month conditioning duration. . . . . . . . . 109

3.11 Surface structures of deionized water samples conditioned for I) 0, II) 24, III) 168, IV) 720, V) 1440 and VI) 2160 hours. . . . . . . . . . 110

3.12 12.5x magnification of the cross section of an i) unconditioned and ii) three-month DI water sample. . . . . . . . . . . . . . . . 111

3.13 Unexposed tool-side surface of an i) unconditioned and ii) three-month DI water sample. . . . . . . . . . . . . . . . . . . . . . 111 
3.14 Reconstructed cross sectional slices of an i) unconditioned sample and a ii) three-month, DI water exposed sample. . . . . . . . . . . . . 113

3.15 Demonstration of void detection; detected voids marked in white. . . 114

3.16 Slice void percentage as a function of length of the scanned volume. . 115

3.17 Average pixel row void percentage as a function of depth of the scanned volume. . . . . . . . . . . . . . . . . . . . 116

3.18 Percentage change of mass of SBS samples as a function of $\sqrt{\text { hours }}$ of exposure. . . . . . . . . . . . . . . . . . 119

3.19 Mass change as a function of conditioning duration for continuous sample; dried samples represented by the last data point. . . . . . . . . 122

3.20 Deposit found on SBS sample following sample evaporation. . . . . . 123

3.21 Approximated Fickian absorption curves. . . . . . . . . . . . 126

3.22 Natural logarithm of diffusivity as a function of inverse temperature. 128

3.23 Percentage mass gain as a function of exposure duration; $100 \%$ represents saturation. . . . . . . . . . . . . . . . . . . . . . . 129

3.24 Percentage change in SBS sample length as a function of conditioning duration; last point represents dimension following drying. . . . . . . 133

3.25 Percentage change in SBS sample width as a function of conditioning duration; last point represents dimension following drying. . . . . . . 133

3.26 Percentage change in SBS sample thickness as a function of conditioning duration; last point represents dimension following drying. . . . . 134

3.27 CME as a function of exposure duration; last point represents CME

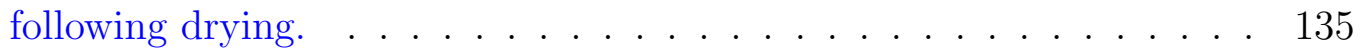

3.28 Change in short beam strength as a function of exposure duration; dried samples represent by individual data points free from the curve.

3.29 Hypothesized inter- and intramolecular contributions to change in short beam strength as a function of exposure duration assuming timeindependent contributions. . . . . . . . . . . . . . . . . 140

3.30 Schematic of SBS sample bag-side surface with hardness measurements numbered; dashed line indicates point of contact of loading nose. . . . 143 
3.31 Change in hardness as a function of exposure duration; dried samples represent by individual data points free from the curve. . . . . . . . . 144

3.32 Comparison of heat flow per gram as a function of temperature during DSC heating cycle for representative nine-week samples. . . . . . . . 146

3.33 Heat flow per gram as a function of temperature during DSC heating cycle for a representative nine-week control sample. . . . . . . . . . 148

3.34 TGA plot of percentage residual mass as a function of temperature. . 149

3.35 TGA plot of percentage residual mass as a function of temperature; initial sample mass represents sample mass following ingressed fluid

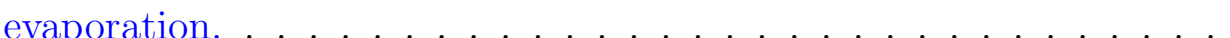

4.1 Thermocouple positions, T1 and T2, on the top-plate of conditioning arrays. . . . . . . . . . . . . . . . . . . 152

4.2 Linear extrapolation of temperature for each sample position, at all tested conditioning durations. . . . . . . . . . . . . . .

4.3 Fractional change in sample mass as a function of extrapolated temperature, presented in terms of conditioning fluid and duration. . . . 155

4.4 Fractional change in flexural strength as a function of extrapolated temperature, presented in terms of conditioning fluid and duration. . 156

4.5 Silicone conditioning gasket following one month of exposure. . . . . 159

4.6 Molecular structure of Poly(dimethylsiloxane). . . . . . . . . . . . 160

B.1 Test tube rack conditioning fluid layout. . . . . . . . . . . . 200

D.1 Stress-strain relationship for 6061-T6, T651 aluminum flexural test; No toe compensation. . . . . . . . . . . . . . . . . . . . . . . 223

D.2 Cross comparison of 3.5x magnification, bag-side flexural sample microscopy performed after 1) 0, 2) 24, 3) 168, 4) 720, 5) 1440 and 6) 2160 hours of exposure to A) deionized water, B) hydraulic fluid, C) ethylene glycol, D) ocean water and E) turbine oil. . . . . . . . . . 224

D.3 Comparison of 3.5x magnification, bag-side flexural sample microscopy performed after 1) 0,2) 24, 3) 168, 4) 720, 5) 1440 and 6) 2160 hours of exposure to air (control). . . . . . . . . . . . . 225

D.4 Change of SBS sample as a function of $\sqrt{\text { hours }}$ of exposure; nonsmoothed. . . . . . . . . . . . . . . . . . . . . 227 
D.5 Mass change of flexural (dashed) and SBS (continuous) samples as a function of $\sqrt{\text { hours }}$ of exposure. . . . . . . . . . . . . . . 229

D.6 Baseline TGA of DI water evaporation, heated at rates of $10^{\circ} \mathrm{C} / \mathrm{min}$, $20^{\circ} \mathrm{C} / \mathrm{min}, 30^{\circ} \mathrm{C} / \mathrm{min}, 40^{\circ} \mathrm{C} / \mathrm{min}$ and $50^{\circ} \mathrm{C} / \mathrm{min} . \ldots \ldots 230$

D.7 Baseline TGA of hydraulic fluid evaporation, heated at rates of $10^{\circ} \mathrm{C} / \mathrm{min}, 20^{\circ} \mathrm{C} / \mathrm{min}, 30^{\circ} \mathrm{C} / \mathrm{min}, 40^{\circ} \mathrm{C} / \mathrm{min}$ and $50^{\circ} \mathrm{C} / \mathrm{min} . \ldots 231$

D.8 Baseline TGA of ethylene glycol evaporation, heated at rates of $10^{\circ} \mathrm{C} / \mathrm{min}, 20^{\circ} \mathrm{C} / \mathrm{min}, 30^{\circ} \mathrm{C} / \mathrm{min}, 40^{\circ} \mathrm{C} / \mathrm{min}$ and $50^{\circ} \mathrm{C} / \mathrm{min} . \ldots 232$

D.9 Baseline TGA of turbine oil evaporation, heated at rates of $10^{\circ} \mathrm{C} / \mathrm{min}$, $20^{\circ} \mathrm{C} / \mathrm{min}, 30^{\circ} \mathrm{C} / \mathrm{min}, 40^{\circ} \mathrm{C} / \mathrm{min}$ and $50^{\circ} \mathrm{C} / \mathrm{min} . \ldots \ldots 233$ 


\section{Nomenclature}

\section{Acronym Definition}

\begin{tabular}{ll}
\hline \hline FAA & Federal Aviation Administration \\
OEM & Original Equipment Manufacturer \\
FRPMC & Fibre-Reinforced Polymer Matrix Composite \\
ACM & Advanced Composite Material \\
CFR & Code of Federal Regulations \\
AC & Advisory Circular \\
NDI & Nondestructive Inspection \\
DOE & Design of Experiment \\
DoF & Degrees of Freedom \\
CV & Coefficient of Variation \\
ANOVA & Analysis of Variance \\
SS & Sum of Squares
\end{tabular}




\begin{tabular}{|c|c|}
\hline FWER & Family-Wise Error Rate \\
\hline FMEA & Failure Modes and Effects Analysis \\
\hline FTIR & Fourier-Transform Infrared Spectroscopy \\
\hline UV & Ultraviolet \\
\hline LVBI & Low-Velocity Blunt Impact \\
\hline BVID & Barely Visible Impact Damage \\
\hline ASTM & American Society of Testing and Material \\
\hline ISO & International Organization for Standardization \\
\hline BSI & British Standards Institution \\
\hline SBS & Short Beam Strength \\
\hline TGA & Thermogravimetric Analysis \\
\hline DSC & Differential Scanning Calorimetry \\
\hline DMA & Dynamic Mechanical Analysis \\
\hline SEM & Scanning Electron Microscopy \\
\hline XPS & X-ray Photoelectron Spectroscopy \\
\hline$\mu$-CT & Micro-Computed Tomography \\
\hline SOP & Standard Operating Procedure \\
\hline UTM & Universal Testing Machine \\
\hline $\mathrm{CNC}$ & Computer Numerical Control \\
\hline
\end{tabular}




$\begin{array}{ll}\text { PTFE } & \text { Polytetrafluoroethylene } \\ \text { CME } & \text { Coefficient of Moisture Expansion } \\ \text { DGEBA } & \text { Diglycidyl ether Bisphenol A } \\ \text { PPGE } & \text { Poly[(phenyl glycidyl ether)-co-formaldehyde] } \\ \text { ROI } & \text { Region of Interest } \\ \text { PDMS } & \text { Poly(dimethylsiloxane }) \\ \text { HPU } & \text { Hydraulic Power Unit } \\ & \\ \text { HSM } & \text { Hydraulic Servo Motor }\end{array}$




\begin{tabular}{|c|c|c|}
\hline Symbol & Definition & Units \\
\hline$T_{g}$ & Glass Transition Temperature & ${ }^{\circ} \mathrm{C}$ \\
\hline$\sigma$ & Stress & $P a$ \\
\hline$P$ & Force & $N$ \\
\hline$A$ & Cross-Sectional Area & $m^{2}$ \\
\hline$L$ & Sample Length & $m$ \\
\hline$b$ & Sample Width & $m$ \\
\hline$h$ & Sample Thickness & $m$ \\
\hline$\varepsilon$ & Strain & - \\
\hline$\delta$ & Mid-span Deflection & $m$ \\
\hline$E$ & Modulus of Elasticity & $P a$ \\
\hline$J$ & Diffusion Flux & $\mathrm{mol} / \mathrm{m}^{2} \cdot \mathrm{s}^{1}$ \\
\hline$D$ & Diffusion Coefficient & $m^{2} / s$ \\
\hline$\varphi$ & Concentration & $\mathrm{mol} / \mathrm{m}^{3}$ \\
\hline$x$ & Distance & $m$ \\
\hline$t$ & Time & $s$ \\
\hline$\Delta m$ & Change in Sample Mass & $g$ \\
\hline $\bar{x}$ & Average Value & Various \\
\hline
\end{tabular}




\begin{tabular}{|c|c|c|}
\hline$x_{i}$ & Single Measured Value & Various \\
\hline$n$ & Number of Specimens & - \\
\hline$s_{n-1}$ & Estimated Standard Deviation & Various \\
\hline $\operatorname{Var}(x)$ & Variance of Parameter $x$ & Various \\
\hline$H_{0}$ & Null Hypothesis & - \\
\hline$H_{1}$ & Alternative Hypothesis & - \\
\hline$F_{0}$ & F-ratio & - \\
\hline \multirow[t]{2}{*}{$q_{\alpha}$} & Percentage Point of Student's Range & $\%$ \\
\hline & Statistic & \\
\hline$\alpha$ & Significance Level & $\%$ \\
\hline$d_{\alpha}$ & Dunnett's Critical Value & $\%$ \\
\hline$M$ & Bending Moment & $N \cdot m$ \\
\hline$I$ & Second Moment of Area & $m^{4}$ \\
\hline$\delta$ & Beam Deflection & $m m$ \\
\hline$\varepsilon$ & Strain & - \\
\hline$F^{S B S}$ & Short Beam Strength & $P a$ \\
\hline$J$ & Diffusion Flux & $\mathrm{mol} / \mathrm{m}^{2} \cdot t$ \\
\hline$C$ & Concentration & $\mathrm{mol} / \mathrm{m}^{3}$ \\
\hline$C_{i}$ & Initial Concentration & $\mathrm{mol} / \mathrm{m}^{3}$ \\
\hline
\end{tabular}




\begin{tabular}{|c|c|c|}
\hline$C_{a}$ & Concentration at time $a$ & $\mathrm{~mol} / \mathrm{m}^{3}$ \\
\hline$m_{\infty}$ & Saturation Mass & $g$ \\
\hline$m_{i}$ & Initial Mass & $g$ \\
\hline$m_{0}$ & Baseline Mass & $g$ \\
\hline$G$ & Moisture Absorption Function & - \\
\hline$M$ & Moisture Content & $\%$ \\
\hline$M_{\infty}$ & Saturation Moisture Content & $\%$ \\
\hline$M_{i}$ & Initial Moisture Content & $\%$ \\
\hline$M_{0}$ & Baseline Moisture Content & $\%$ \\
\hline$D_{0}$ & Permeabilty Index & $m^{2} / s$ \\
\hline$E_{d}$ & Activation Energy of Diffusion & $J / m o l$ \\
\hline$T_{m}$ & Melt Temperature & ${ }^{\circ} \mathrm{C}$ \\
\hline$\Delta E$ & Change in Electron Energy & $J$ \\
\hline$\lambda$ & Wavelength & $m$ \\
\hline
\end{tabular}

\begin{tabular}{lll}
\hline Symbol & Constant & Value \\
\hline \hline$h$ & Planck's Constant & $6.626 \times 10^{-34} \mathrm{~J} \cdot \mathrm{s}$ \\
$c$ & Speed of Light & $2.998 \times 10^{8} \mathrm{~m} / \mathrm{s}$ \\
$R$ & Universal Gas Constant & $8.314 \mathrm{~J} / \mathrm{mol} \cdot \mathrm{K}$ \\
\hline
\end{tabular}

xxiv 


\section{Chapter 1}

\section{Introduction}

This chapter presents the motivation for this thesis research, describes the thesis report structure, offers a background into engineering composites - with focus given to their certification and testing methodologies - and presents the scope of research. Subsequently, a literature review is presented which describes environmental degradation of mechanical properties of aerospace composites, and aims to identify research gaps pertinent to exposures which may be detrimental to composite structures in an aircraft interior. Based on identified gaps, a sequence of testing campaigns were proposed, defining research objectives which are feasible based on logistical and temporal constraints.

\subsection{Motivation}

Continued intransient performance of structural aircraft components is paramount; collapse or fracture of critical, load-bearing structure can lead to catastrophic failure and loss of life. Consequently, regulatory bodies, such as the United States 
Department of Transportation Federal Aviation Administration (FAA), have established stringent regulations which aid in ensuring continued airworthiness of aircraft components. To certify continued airworthiness, original equipment manufacturers (OEMs) must provide experimental evidence which demonstrates material design values, or allowables, are attained with a high degree of confidence in the appropriate critical environmental exposures to be expected in service [3]. Hence, OEMs must devise and perform experiments which emulate realistic, in-service conditions to predict component service-life, define design allowables, and to recommend inspection frequencies.

Fibre-reinforced polymer matrix composites (FRPMCs) are widely used for structural aerospace applications due to their high specific mechanical properties. However, FRPMCs demonstrate a high degree of variability in mechanical properties, when compared to conventional isotropic materials, due to diversity and complexity of layup processes; anisotropicity; heterogeneity; and matrix sensitivity to environmental exposure [3]. Hence, while FRPMCs offer superior tailorability and versatility, they require additional consideration to ensure they effectively reach their intended service life.

This work is a component of collaborative research between Carleton University and General Dynamics Missions Systems - Canada (hereafter referred to as the 'industrial partner') to evaluate the degradation of mechanical properties in FRPMCs due to environmental exposures common to maritime aircraft interiors. This study aims to identify environmental exposure which, realistically, have the propensity to interact with a FRPMC structure utilized in a maritime aircraft interior; devise an empirical and pragmatic testing campaign to quantify the effects of identified routes of exposures on the mechanical properties of a FRPMC; and perform the prescribed campaign, examining both individual and synergistic effects. 


\subsection{Organization of Thesis}

This thesis is arranged into six chapters: Introduction, Methodology, Results, Experiment Evaluation, Conclusions and Recommendations. Following the Introduction, Methodology presents the experiment proposal, methods employed to achieve outlined objectives, accompanying method-specific theory and the testing and validation of fixtures designed. The Results section presents obtained results and associated discussion for the two test campaigns implemented. Experiment Evaluation details obstacles encountered from novel, nonstandard experimental methods performed, their influence on testing and potential biases on results. Lastly, overarching inferences from the results obtained are presented in Conclusions and recommendations for future work - further validating or building from the work performed - are presented in the Recommendations. Supplementary material for has been presented in five appendices following the body of the report.

\section{$1.3 \quad$ Background}

A composite material, hereafter referred to as a composite, is a material composed of two or more distinct, heterogeneous materials. With such a broad classification, many common materials, including wood and reinforced concrete, may be classified as composites [8]. Engineering composites have been designed with the intention of improving one or more of the constituent material's properties through the integration of additional materials which possess the property of interest. Properties of interest can vary depending on application but some common examples include strength, stiffness, melt temperature, electrical conductivity, thermal conductivity, coefficient of thermal expansion, and chemical resistance. The number of parameters which 
engineers can optimize through composite design are numerous making consideration of their application important for a wide range of engineering work.

\subsubsection{Advanced Composite Design}

Advanced composite materials (ACMs) are commonly selected for structural applications. While they may take a variety of forms, traditional ACMs are composed of two distinct phases - a matrix and a fibre. The matrix, or continuous phase, is the phase in which the fibre, or reinforcement phase is embedded. While it is conventionally understood that the fibre phase - with their high strength and stiffness - is used to enhance the mechanical properties of the matrix material, it is the synergistic combination of the two phases which, when coupled, produce a superior material benefiting from the contributions of each phase; matrix material allowing load transfer between fibres. Embedded fibre may be continuous throughout the matrix - oriented unidirectionally, in two-dimensional or three-dimensional laminates - or may be discontinuous and randomly distributed throughout. Figure 1.1 illustrates a top-down view of three common fibre orientations.

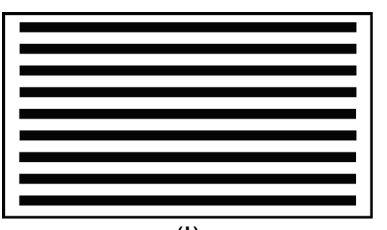

(I)

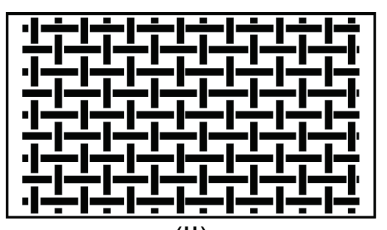

(II)

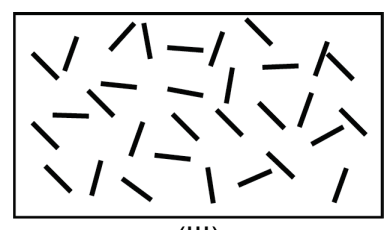

(III)

Figure 1.1: Examples of I) unidirectional II) woven and III) short fibre, fibrereinforced composites.

ACMs are classified by both their matrix and fibre materials, and may be composed of numerous combinations of conventional, homogeneous materials such as polymers, ceramics and metals, as desired for a given application. Today, ACMs are a fundamental material within the field of engineering. They are used in a wide variety of 
applications including civil infrastructures, marine vessels, sporting goods, automobiles and aerospace.

Driven by post-war technological advancement following World War II [9], FRPMCs are one of the most common types of ACMs for aircraft application. FRPMCs have gained significant interest in aerospace due to their high specific properties, which allow reduced fuel consumption through weight reduction, and tailorability - attributed to their compliance prior to cure - which allows for complex part design and improved economy through a reduced part count. Unlike conventional metal alloys, FRPMC are often designed in conjunction with the structural component which they compose. While this versatility is highly beneficial, resulting compositional variability introduces unique challenges when it comes to the evaluation and certification of FRPMC-containing aircraft.

\subsubsection{Composite Certification}

In order for an aircraft component to be considered airworthy, that is that it "conforms to its type design and is in a condition for safe operation" [10], it must be subjected to rigorous testing which validates it will sustain functionality for the duration of its prescribed service life. The FAA provides airworthiness certification, granting authorization for the operation of aircraft in flight in the United States of America. As the largest authority in aviation, certification guidelines defined by the FAA form the cornerstone of aviation best-practices and are widely acknowledged as an international standard from which much of the world's civil aviation regulation is based. While the FAA does not certify individual aircraft components, they do provide acceptable means by which to demonstrate compliance with the US Code of Federal Regulation (CFR) for airworthiness certification. FAA advisory circular 
(AC) 20-107B offers such means for composite aircraft structures which utilize fibre reinforced materials. In order to ensure an economical, yet comprehensive testing campaign (a collection of tests performed in order to achieve an objective or address one or more hypotheses), AC 20-107B recommends a "building block" approach. As illustrated in Figure 1.2, this approach follows incremental testing, from coupons to component, with the greatest number of tests taking place at the coupon level, requiring less tests at each sub-sequent stage with increasing complexity. In this manner, results obtained from relatively inexpensive coupons testing can be used to influence design choices at sub-component and component phases which require more complex and expensive test specimens.

Tests at each level are performed with the intention of establishing design allowables. Materials properties are variable; as such, the probabilistic distribution of a material value must be considered in structural design. This is particularly important for composite materials which demonstrate considerable variability, both as a result of composition and employed fabrication processes. Design allowables are material property values which are specified based a probability basis. A-basis allowables specify the threshold value for which there is a $99 \%$ probability with a $95 \%$ confidence interval that the component of interest will exhibit an equivalent or greater material value [11]. Likewise, B-basis allowables specify the threshold value for which there is a $90 \%$ probability with a $95 \%$ confidence interval that the component of interest will exhibit an equivalent or greater material value [11].

Consideration of design allowables established from early tests, in conjunction with the required load carrying capacity and applied safety factor, dictates materials selection and the application of safe-life or fail-safe design strategies at the sub-component and component stages. If a material exhibits an A-basis allowable which is acceptable given anticipated service loads, a safe-life strategy may be employed [12]. Safe-life 


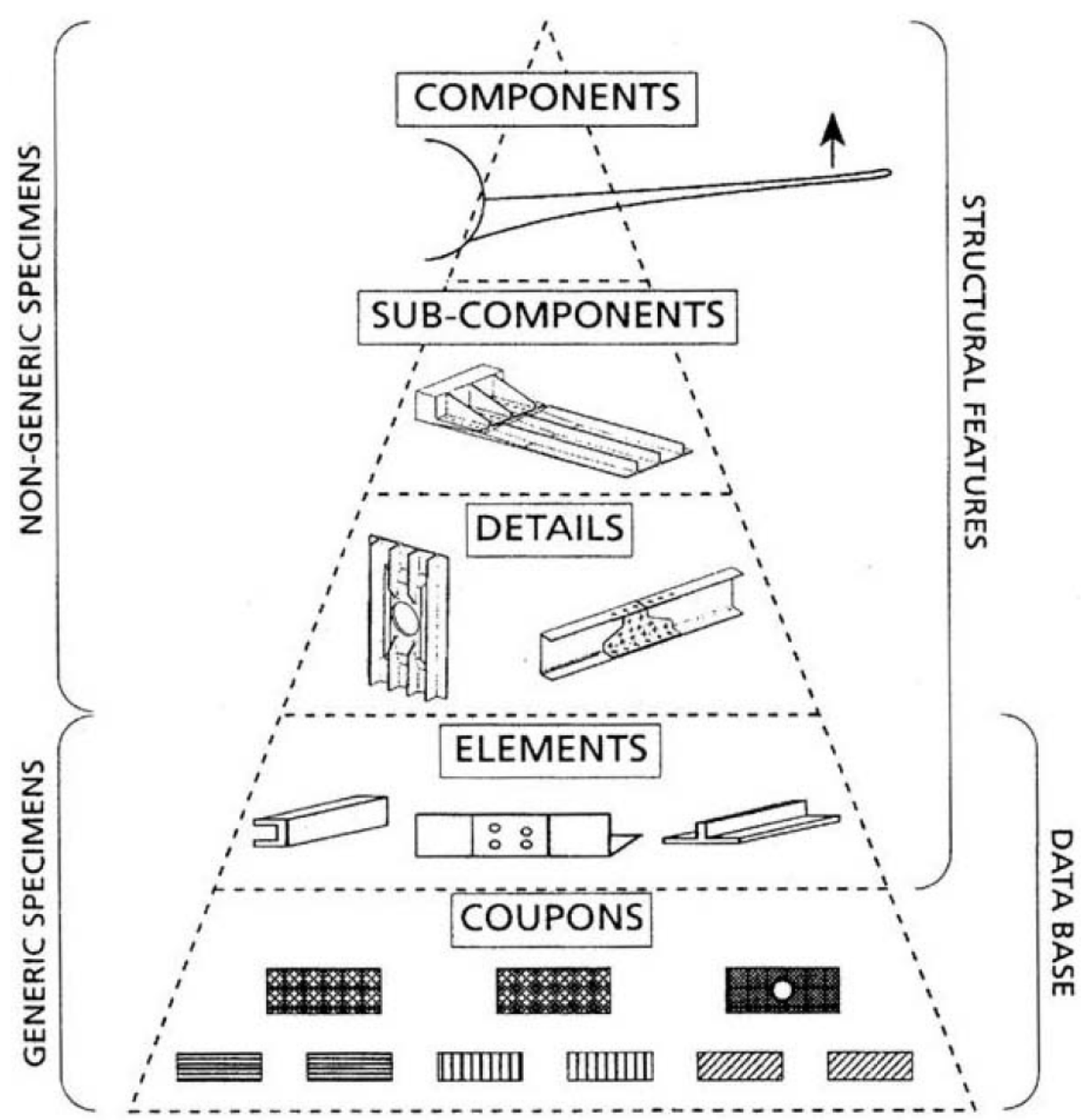

Figure 1.2: The hierarchy of the building block approach used to incrementally assess aircraft components, adapted from [3].

components are designed to survive their service life, without failure, relying on a single load path. If a material does not exhibit an acceptable A-basis allowable but exhibits an acceptable B-basis allowable, a fail-safe strategy may be employed [3,12]. Fail-safe components are designed with redundant load paths such that if the primary load path were to fail, applied load would safely be distributed to other load carrying members. Such practices are employed to reduce the risk of failure of flight-critical structures (primary structures) in flight. While both safe-life and fail-safe design are used in aerospace, their is a desire to reduce the need for redundant members to minimize materials cost and overall mass. Additionally, redundant members may not 
be feasible in instances where design has a substantial role in functionality, as is the case with flight control surfaces.

The extent of testing required at each level varies significantly depending on a number of component-specific factors (e.g. criticality to flight safety). Testing must consider manufacturing variability, material variability, environmental exposure, structural protection and structural details [3], and how these factors may effect both the static and dynamic loading capabilities of the component, synergistically, over the duration of its service life. Hence, there is a need to both accelerate the rate of accumulation of factors contributing to degradation and test the effects of a large number of potentially contributing factors.

\section{Accelerated Ageing}

Accelerated ageing is a common experimental technique which allows examination of prolonged material exposure to a variety of factors in a fraction of the time that would be required to test a material through its intend service life [13]. Accelerated ageing can be accomplished in a variety of ways depending on the factor under investigation. This can include increasing chemical concentration; increasing or decreasing temperature; increasing radiation intensity; increasing loading; etc. For example, when examining the detrimental effects of chemicals on FRPMC, increasing the temperature of environment can help accelerate the observed effects, both through increasing the rate of fluid ingress into the material and by increasing the reaction rate by allowing a greater number of species to exceed reaction energy barriers. Likewise, fatigue testing, which looks at the change in a material's properties through repeated loading,

can be accelerated by either increasing the rate of loading (cycles/unit of time) or by increasing the force imposed per cycle. 
When employing accelerated ageing, it imperative to have a method of correlating the observed, accelerated rates of exposure with the in-service rate of exposure, allowing life-time predictions based on observed degradation rates. Additionally, it is important that accelerated ageing does not introduce confounding mechanisms which could aid or hinder the material's degradation [13]. For example, in his work on thermal oxidation of composites materials, Tsotsis concluded that when accelerating thermally oxidative processes, emulation of oxidation may be more effectively achieved by increasing oxygen pressure at lower temperature rather than primarily focusing on temperature elevation [14]. Accelerated ageing is a valuable tool for estimating component service-life and proscribing inspection and maintenance frequency.

\section{Nondestructive Inspection}

While accelerated ageing is useful for characterizing age-dependent degradation, nondestructive inspection (NDI) is required for both quality control of manufactured and repaired components and to ensure component longevity through routine inspection in-service. As the name suggests, NDI allows for the inspection of components without the need to destructively evaluate (test) stress-strain values which, is impractical for in service components. While NDI conventionally can not evaluate mechanical properties, it can detect inconsistencies or defects in composites which are known to diminish them. Such defects include voids, wells, holes, cracks, channels, delamination and disbonding.

One of the primary and most practiced methods of NDI is visual inspection. Visual inspection involves trained personnel visually inspecting, without any aids, a composite's surface for defects which may compromise structural integrity. Visual 
inspection is the most common form of NDI as it is inexpensive and widely applicable as, by airworthiness design philosophy, many material irregularities which pose concern are visible [8]. For internal or inaccessible surfaces, a number of other NDI techniques and equipment exist; these include tap testing, ultrasonic inspection, $\mathrm{x}-$ ray methods, Eddy Current inspection, thermography, bond testers, moisture meters, interferometry and shearography [8]. While tap testing and ultrasonic inspection are most common, each technique offers its own set of advantages and disadvantages and may be applicable based on the anticipated defect type and orientation; material type; and required resolution. Unfortunately, without visual indication, it is difficult to justify the implementation of alternative NDI techniques. This raises the concern that an undetectable defect may propagate internally, compromising structurally integrity and complicating repair once detected. Consequently, rigorous material testing which quantifies degradation from all conceivable means of in-service structural degradation is preeminent.

\subsubsection{Design of Experiment}

In order to quantify changes to structural properties, testing must be performed which addresses all conceivable means of in-service structural detriment. Ensuring design allowables are established for all factors, both independently and synergistically, can be an arduous, time consuming and expensive exercise requiring a considerable number of samples in order to demonstrate statistical significance.

Design of experiment (DOE) is the process of systematically associating factors of interest (independent variables) with variation in parameters of interest (dependent or response variables) and is essential for systems with a large number of degrees of freedom (DoF). Factorial design is a DOE methodology which promotes improved 
testing efficiency by reducing the number of tests required to demonstrate significance through systematic statistical analysis [15]. Factorial design allows experimenters to vary multiple factors, together, rather than individually, allowing the evaluation of interactions between factors. In this manner, a test can simultaneously gain information regarding the effects of changing several independent variables, reducing the number of independent tests required to obtain significant results. Factorial design offers a $\frac{k}{2}+\frac{1}{2}$ improvement in efficiency over traditional one-factor-at-a-time experimentation [15] for experiments with two factor levels, where $k$ is the number factors under investigation.

The benefits of factorial design may best be illustrated by an example. Simple factorial experiments (with three factors or less) can be illustrated geometrically with lines and nodes to help visualize the relationships between tests. Figure 1.3 illustrates a simple hypothetical experiment considering the effects of cure parameters on the tensile strength of a FRPMC.

In this experiment, three factors are under investigation; matrix material, cure pressure and cure time. For each factor there are two levels being evaluated, in this example, epoxy and polyester matrix materials are being considered at two pressure levels, 40 psi and 80 psi, over two durations, 30 minutes and 60 minutes. In order to test every combination of these factors by factorial design, there must be eight tests performed, each indicated by a node. Table 1.1 depicts each of the required experimental trials as a combination of pluses $(+)$ and minuses $(-)$ indicating one-of-two potential levels. Not only would such a test have two-times the relative efficiency of a comparable one-factor-at-a-time experiment, it also offers additional information about effects of higher-order interactions on the response variable (tensile strength).

Factorial experiments are characteristically represented by the notation $(n) x^{k}$ where 


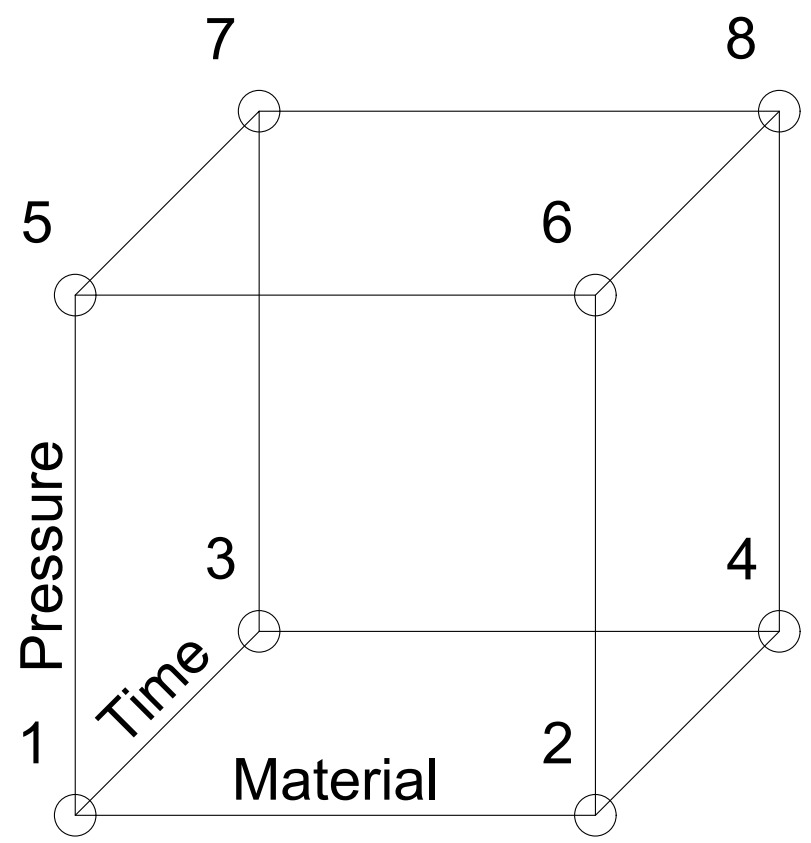

Figure 1.3: Geometric illustration of factorial design.

$x$ is the number of levels and $n$ is the number of repetitions. Evaluation of this expression reveals the total number of tests required for a single experiment. Hence, for this example, if there are five repetitions of each test required, there will be 40 tests in total.

For tests comparing a large number of factors, an exponentially larger number of tests will be required to perform a full factorial design. For experiments of this nature, fractional factorial design may be applied. Fractional factorial design sacrifices the identification of higher-order interactions between factors as a means of reducing the total number of tests required [15]. This method should only be practiced when it is anticipated that prominent effects will be sparse, that is, of the many factors under investigation, only a few will exhibit a quantitatively significant effect. When considering structural analysis, this method is particularly effective at low-level coupon testing as a means of determining which independent variables will effect material 
Table 1.1: Potentially testable factor-level combinations.

\begin{tabular}{ccccccccc}
\hline Factor & 1 & 2 & 3 & 4 & 5 & 6 & 7 & 8 \\
\hline Material & - & - & + & + & - & - & + & + \\
Time & - & + & - & + & - & + & - & + \\
Pressure & - & - & - & - & + & + & + & + \\
\hline
\end{tabular}

strength or modulus values. Hence, fractional factorial design allows for efficient experimentation, limiting inconsequential testing, fostering faster concentration on critical factors.

\subsection{Statistical Analysis}

Due to the probabilistic nature of material properties and, specifically for composites, inconsistencies in fabrication processes, material testing relies heavily on statistical analysis in order to draw credible, meaningful conclusions. Equations 1.1 to 1.4 allow calculation of four values essential to statistical analysis: arithmetic mean, $\bar{x}$ (or average), sample standard deviation, $s_{n-1}$, variance, $\operatorname{Var}(x)$, and coefficient of variation, $C V$, where $x$ is a value of some arbitrary parameter of interest and $n$ is the number of given values.

$$
\bar{x}=\frac{1}{n} \sum_{i=n}^{n} x_{i}
$$




$$
\begin{gathered}
s_{n-1}=\sqrt{\frac{\left(\sum_{i=n}^{n} x_{i}^{2}-n \bar{x}^{2}\right)}{n-1}} \\
\operatorname{Var}(x)=s_{n-1}^{2} \\
C V=\frac{s_{n-1}}{\bar{x}} \times 100
\end{gathered}
$$

While the term "average" is colloquial, formally it is one of a number of methods of describing the central tendency of a group of values. Conversely, standard deviation and variance are used to describe the dispersion of a set of values, or how values within a set are distributed with respect to the average. As standard deviation is the square of variance both values may be used to describe the variability of a data set. However, because standard deviation is expressed in the same units as the mean, it offers a more intuitive meaning when discussing results, whereas variance's associative property makes it more useful for successive calculations (i.e. analysis of variance). Lastly, the coefficient of variation is a measure of the deviation with respect to the average. The CV offers an added benefit of being dimensionless, allowing for a more meaningful comparison of the variation in sample groups which have different units or notably different means (e.g. flexural strength of two, dissimilar materials). 


\subsubsection{Hypothesis Testing}

Hypothesis testing is a method which allows the formation of inferences regarding a sample population based on observed data from a subset, or sample of a population [16]. Hypothesis testing aims to address a research hypothesis, of which the truth is unknown, through the comparison of one or more sample sets to either a population or each other. From the research hypothesis, a null hypothesis, $H_{0}$, and an alternative hypothesis, $H_{1}$, are formed: the null, a hypothesis that there is no relationship between measured phenomena, and the alternative, a hypothesis that there is a relationship between measured phenomena. Hypothesis testing functions to validate or invalidate the null hypothesis. If statistical testing reveals significance, that is, that the observed results are unlikely to have occurred given the null hypothesis be true, the null hypothesis is rejected in favour of the alternative. Conversely, if significance is not established, the null hypothesis is accepted. Significance is determined based on a defined significance level, a probability values (p-value) below which the probability of a observation is unlikely given acceptance of the null hypothesis.

While the selection of a significance level is discretionary, convention commonly defines either a p-value of $5 \%$ or $1 \%$ as significant based on the work of Fisher $[17,18]$. In this context, considering a p-value of $1 \%$, there is a $1 \%$ probability of the observed phenomena occurring by random chance. Selection of p-value is often decided based on the consequences of incorrectly rejecting or accepting the null hypothesis. Incor-

rectly rejecting the null hypothesis, known as a type I error, is a false positive - a correlation is observed for the sample which is not observable for the population. Conversely, incorrectly accepting the null hypothesis, known as a type II error, is a false negative - a correlation is not observed for the sample which is observable for the population. For a given circumstance, inadvertently achieving either type I or type II error may be less favourable. In the context of environmental degradation of 
aerospace components, type I error is less adverse as it is more conservative-while falsely identifying a correlation between exposure and degradation may seemingly be more costly due to constraints imposed (i.e. additional testing, limited service life, increase inspection frequency, etc.) it is less likely to result in the loss of human life. Hence, for this work, selection of a higher p-value may be preferential, ensuring the correct identification of degradative factors.

\subsubsection{Analysis of Variance}

There are a number of test statistics used for hypothesis testing, each applicable based on calculability of sample distribution. The calculability of sample distribution depends on a number of factors including population distribution, number of samples, number of sample groups, sample size, test intention and knowledge of population mean. Analysis of variance (ANOVA) is a test statistic, based on the F-test, which allows for a general comparison of the means of sample groups. ANOVA is well suited for multi-factor, multi-sample group testing as it allows inferences to be drawn regarding whether or not a factor, or interaction of factors, causes a statistically significance effect on the response variable. Hence, ANOVA is a useful method for reducing the number of tested factors in experiments with a large number of independent variables.

The central concept of ANOVA involves comparing the variance within sample groups (error) to the variance between sample groups (treatment response) and determining significance based on a comparison of the ratio of the two to a significance level. ANOVA can be applied to experiments considering any number of factors however, to retain pertinence to the work discussed, a two-factor, or two-way ANOVA will be considered. Describing the observations of a two-factor experiment with a fixed-effect model (i.e. fixed treatments are applied to samples in order to evaluate their effect 
on the response variable), the observed response, $y_{i j k}$, may be expressed as

$$
y_{i j k}=\mu+\tau_{i}+\beta_{j}+(\tau \beta)_{i j}+\epsilon_{i j k}\left\{\begin{array}{l}
i=1,2, \ldots, a \\
j=1,2, \ldots, b \\
k=1,2, \ldots, n
\end{array}\right.
$$

where $\mu$ is the overall mean effect, $\tau_{i}$ is the effect of the $i$ th level of factor $\mathrm{A}, \beta_{j}$ is the effect of the $j$ th level of factor $\mathrm{B},(\tau \beta)_{i j}$ is the effect of the interaction between $\tau_{i}$ and $\beta_{j}, \epsilon_{i j k}$ is the random error [15] (unpredictable error inherent to a test), $a$ corresponds to the levels of factor A, $b$ corresponds to the levels of factor $\mathrm{B}$ and $n$ corresponds to the number of replicates of each test. $\tau_{i}$ and $\beta_{j}$ are defined as deviations from $\mu$. As there are multiple factors as well as their interaction, multiple hypothesis tests are required to make inferences about significance of each. For a test intended to evaluate all factor and levels for a response, such hypotheses take the form

$$
\begin{array}{r}
H_{0}: \tau_{1}=\tau_{2}=\cdots=\tau_{a}=0 \\
H_{1}: \text { at least one } \tau_{i} \neq 0 \\
H_{0}: \beta_{1}=\beta_{2}=\cdots=\beta_{a}=0 \\
H_{1}: \text { at least one } \beta_{j} \neq 0
\end{array}
$$




$$
\begin{array}{r}
H_{0}:(\tau \beta)_{i j}=0 \text { for all } i, j \\
H_{1}: \text { at least one }(\tau \beta)_{i j} \neq 0
\end{array}
$$

To test these hypotheses, the variance of $\tau_{i}, \beta_{j}$ and $(\tau \beta)_{i j}$ must be compared to the variance of random error in what's known as an F-ratio, $F_{0}$. From equations 1.2 and 1.3, it can be observed that variance of a data set may be represented as a ratio of its sum of squares (SS) to DoF. In ANOVA, the total sum of squares $\left(S S_{T}\right)$ is the sum of squared differences of each observation of the response variable with the mean response and may be represented by a summation of the SS between sample groups and the SS within sample groups. For two-way ANOVA, SS between sample groups must consider both factors and their interactions, hence $S S_{T}$ may be represented as

$$
S S_{T}=S S_{A}+S S_{B}+S S_{A B}+S S_{E}
$$

where $S S_{A}$ is the SS due to factor A, $S S_{B}$ is the SS due to factor B, $S S_{A B}$ is the SS due to their interaction and $S S_{E}$ is the SS due to error (SS within sample groups). Utilizing this relationship and the DoF of each effect, values of $F_{0}$ pertaining to each of the hypotheses may be deduced. DoF for each effect are expressed in Table 1.2. Equations A.1 through A.5 in the Appendix A describe the constituent SS equations for the effects comprising 1.9 .

Once $F_{0}$ has been calculated, its magnitude can be compared against the percentage points of the $\mathrm{F}$ distribution, which are commonly tabulated and presented based on significance level. If $F_{0}$ is found to be greater than the percentage point for a given combination of numerator and denominator DoF values, the observed response is said 
Table 1.2: Effects and associated DoF for two-way ANOVA.

\begin{tabular}{ll}
\hline Effect & DoF \\
\hline$A$ & $a-1$ \\
$B$ & $b-1$ \\
$A B$ interaction & $(a-1)(b-1)$ \\
Error & $a b(\mathrm{n}-1)$ \\
Total & $a b n-1$ \\
\hline
\end{tabular}

to be statistically significant and the null hypothesis can be rejected. The larger the value of $F_{0}$, the more significant the result and less chance there is of achieving type I error.

\subsubsection{Tukey's test and Dunnett's Test}

Where ANOVA falls short is in its specificity; it does not allow comparison of specific sample group means and thus, cannot be used to ascertain relative significance of specific tested factor-level combinations. Given an implementation of ANOVA which rejects the null hypothesis, pairwise comparison of means may be of benefit, thus necessitating additional analysis post hoc. There are a number of test statistics which allow the comparison of two samples means. As the number of sample sets being compared increases, the chance of making an error in choosing to accept or reject the null hypothesis increases. The probability of making one or more type I errors is know as the family-wise error rate (FWER). Hence, tests which perform multiple pairwise comparisons of the mean must be designed to limit FWER [15]. Two tests which aim to do this are Tukey's test and Dunnett's test. Both Tukey's test and Dunnett's test are similar in implementation to the two-sample t-test, but are designed to be more robust for multi-sample experiments. Tukey's test compares all pairs of means in an 
experiment while Dunnett's test compares the means of all sample sets to the mean of a control, thus hypotheses for both test maybe represented as

$$
\begin{aligned}
& H_{0}: \mu_{i}=\mu_{j} \text { for all } i \neq j \\
& H_{1}: \mu_{i} \neq \mu_{j} \text { for all } i \neq j
\end{aligned}
$$

$$
\begin{aligned}
& H_{0}: \mu_{i}=\mu_{a} \\
& H_{1}: \mu_{i} \neq \mu_{a}
\end{aligned}
$$

respectively, where $\mu_{a}$ is the sample mean of the control sample set. Tukey's test is based on the studentized range statistic. For two sample groups of unequal size, if the absolute value of the difference in sample means, $\left|\overline{y_{i}}-\overline{y_{j}}\right|$, is greater than

$$
T_{\alpha}=\frac{q_{\alpha}(a, f)}{\sqrt{2}} \sqrt{M S_{E}\left(\frac{1}{n_{i}}+\frac{1}{n_{j}}\right)}
$$

where $q_{\alpha}(a, f)$ is the percentage point of the student's range statistic, with significance level $\alpha, a$ sample means, $f$ DoF, and $M S_{E}$ is the mean square error, then the result is significant. Dunnett's test, derived from Tukey's test, is similar in structure and application to Tukey's. However, because it is strictly for comparisons of multiple means with a control, it may use the student's t-distribution, allowing for narrower confidence intervals [19]. Comparing a treatment group to the control, if the absolute value of the difference in sample means, $\left|\overline{y_{i}}-\overline{y_{a}}\right|$, is greater than 


$$
D_{\alpha}=d_{\alpha}(a-1, f) \sqrt{M S_{E}\left(\frac{1}{n_{i}}+\frac{1}{n_{j}}\right)}
$$

where $d_{\alpha}(a-1, f)$ is the critical value for Dunnett's test based on student's tdistribution, with significance level $\alpha, a-1$ sample means and $f$ DoF, then the result is significant. Using either the Dunnett's test for comparison of treatment groups with a control or Tukey's test for cross-treatment group comparison allows for statistically robust pairwise comparisons, particularly useful for large DOE work observing the relative significance of tested parameters.

\subsection{Scope of Research}

This work attempted to evaluate the effects of environment-specific factors, typical of the standard working environment of a maritime aircraft interior, on the static structural properties (i.e. strength and stiffness) of a FRPMC material composing a noncritical structure. To do this, secondary research (i.e. literature review) was performed to; i) predict environmental factors common to a maritime aircraft interior; ii) determine which of the identified factors have the propensity to adversely effect structural properties of a FRPMC; and iii) identify what analytical techniques, destructive and nondestructive, are viable to for quantifying these effects and diagnosing their existence in service, respectively. Following this, primary research pragmatically tested the factors identified on a FRPMC, through the application of DOE and factorial design methods, coupled with statistical analysis, to efficiently quantify their effects.

This study examined a monolithic, woven, carbon fibre-reinforced epoxy while maintaining a consistent lay-up, composition and cure process for a given test campaign. Effort was not made to investigate effects of varying the composite's type, lay-up, 
composition, cure process, additives or coatings on the associated degradation. No work was performed investigating effects of degradation on the composite's adhesively bonded joints, whether it be between monolithic panels or monolithic sandwich panels and their core structure. The composite was exposed only to environmental factors which are anticipated to be present in the interior of maritime-based military aircraft and those which are anticipated to cause degradation as described in literature. Testing was not targeted dynamic mechanical properties, including damage tolerance and fatigue, brought about by anticipated, cyclic in-service loading. Furthermore, this study did not investigate residual stresses, brought about through fabrication, differences in thermal expansion of bound components, or structural details (i.e. holes and joints) and thus, as an extension, did not examine repaired components or crashworthiness. Testing was restricted to the coupon-level, material properties.

\subsection{Literature Review}

\subsubsection{Review Taxonomy}

The goal of this literature review was to collect and summarize information from past studies on the effects of environmental degradation on FRPMCs, and applicable analysis techniques, to identify knowledge gaps which may be bridged through further research. The focus of the review was to identify, compare and contrast research methodologies used and outcomes observed through the study of composite degradation. The review intended to maintain a neutral perspective and looked to draw from a representative sampling of relevant work contained in journal articles, books, theses, and conference papers. It was decided that the review would be organized in a conceptual manner and would be prepared for specialized scholars interested in 
evaluation of FRPMC degradation. This taxonomy was devised based on Cooper's 1988 article, Organizing knowledge syntheses: A taxonomy of literature review [2], and a has been summarized in Table A.1 of Appendix A.

\subsubsection{Review Structure}

The literature review's structure was based on J.J. Randolph's 2009 article, A Guide to Writing the Dissertation Literature Review [20]. Randolph takes a systematic approach to secondary research, making it desirable to achieve clarity and reproducibility. Randolph's structure involves five primary stages including problem formulation; data collection; data evaluation; analysis and interpretation; and public presentation.

\section{Problem Formulation}

The goal of the Problem Formulation stage was to determine what questions the literature review would attempt to answer. The explicit questions the literature review intended to address are as follows:

1. What environmental factors are common to a maritime aircraft interior?

2. Which of the identified factors have the propensity to adversely effect structural properties of a FRPMC?

3. What analytical techniques, both destructive and nondestructive, are viable to for quantifying these effects and diagnosing their existence in service? 


\section{Data Collection and Evaluation}

The goal of the Data Collection and Evaluation stage was to collect qualitative and quantitative results relevant to the formulated questions in a predefined, systematic manner which would allow another researcher attempting to answer the same questions to obtain the same answers. This was done by first defining a set of criteria which states what content will be included in, or excluded from, the review. These criteria are as follows:

- The article has been written in or translated to English

- The article was published before the end of July 2017

- The article discusses a neat resin; fibrous, polymer composite laminate; or laminate-honeycomb structure composed of materials common to the aerospace industry

- The article discusses some form of degradation to the neat resin or composite and/or a quantitative or qualitative means by which to evaluate their degradation in terms of structural integrity

- The article does not primarily focus on a composite with an added, novel feature (e.g. use of an uncommon matrix resin, fibre, lay-up technique, filler material)

- The article does not focus on discontinuous fibre or particle filler materials

Following an algorithm of paper discovery and analysis, articles were selected for inclusion in the review based on their relevance to the investigation. Papers which were selected then had relevant information extracted and presented in a code book-a spread sheet sorted by areas of inquiry. Throughout this process, logs were kept 
of both literature keywords searched and papers which where unattainable through Carleton University's library resources, but were potentially relevant. Extracted codebook categories are listed in Table A.2 of Appendix A.

\section{Analysis, Interpretation and Presentation}

Based on the papers assessed, collected data was then analyzed for trends and discrepancies which were used to propose experiments to bridge identified knowledge gaps. The results of the literature review and proposed experimentations were then presented to scholars in the field (thesis supervisors) to ensure the identified gaps were accurate and the proposed research was sound. The results which were obtained are presented categorically in the subsections which follow and a review summary is detailed thereafter.

\subsubsection{Environmental Factors}

To first assess the realistic capacity of environmental factors to degrade the mechani-

cal properties of the material in question, an exploratory, simplified failure modes and effect analysis (FMEA) was performed. Traditionally, FMEA is based on historical accounts of structure failure, however, as the structure of interest is not primary nor conventionally composed of a FRPMC, archival data is unavailable. Hence, probability and severity values have been assigned based on the known, and anticipated, working environment of the FRPMC structure determined through consultation with the industrial partner. Table 1.3 presents all potential macroscale sources of degradation, as identified by literature $[3,8,21,22]$ paired with values of Probability, how likely the FRPMC structure is to be exposed to the factor, and Severity, if the FRPMC 
structure was exposed to the factor, how detrimental the factor would be to the structure's function. Probability and severity range from one to five, where one suggests exposure is unlikely and resulting damage is insignificant and five suggests exposure is certain and resulting damage is catastrophic. Factors were then assigned a risk level from the multiplication of their probability and severity. It was decided that factors given a risk factor of eight or higher would be investigate further in secondary research, focusing subsequent efforts by removing factors deemed inconsequential due to their low probability of exposure or low severity of damage. 


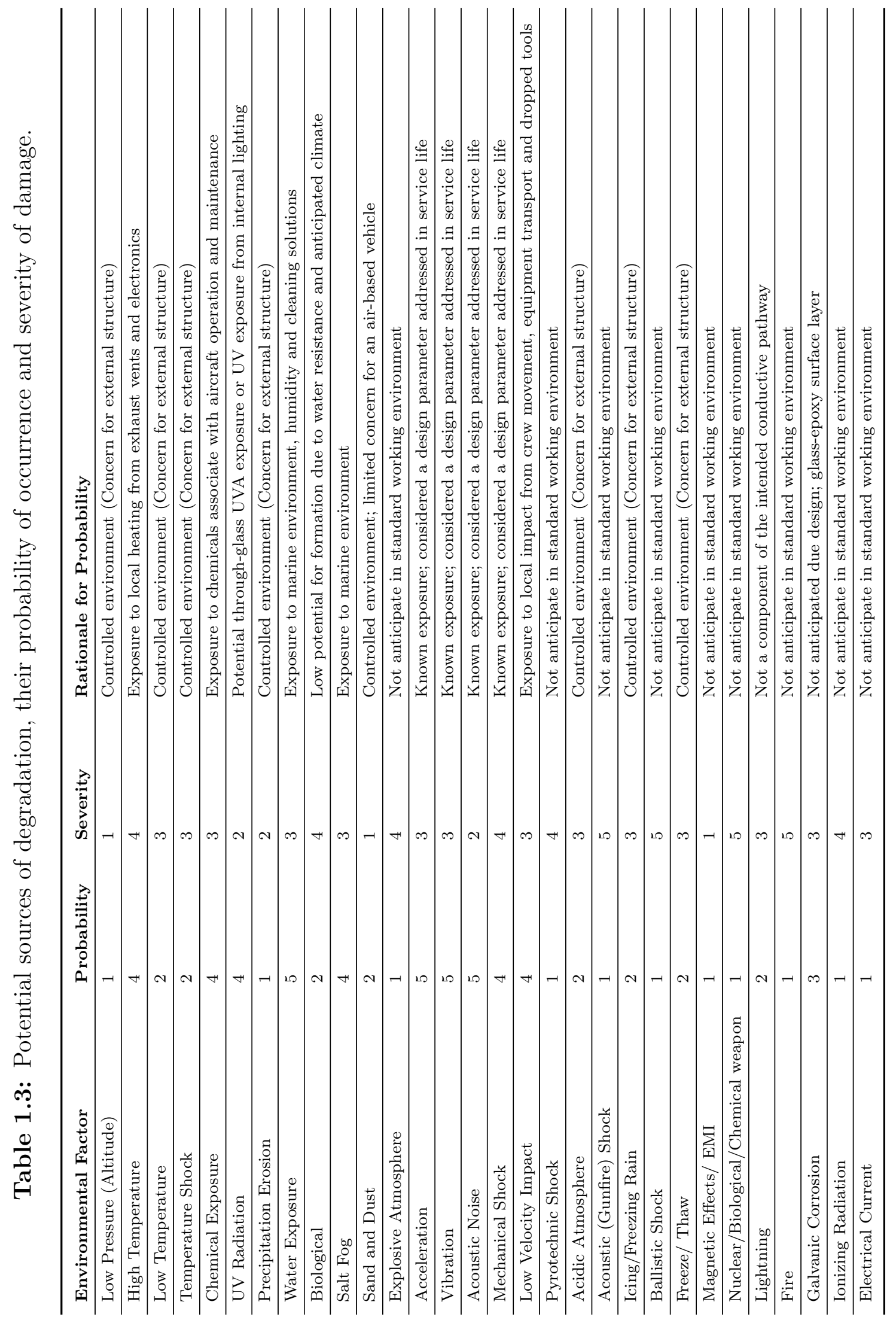


From this, the following factors have been identified as being potentially detrimental to the FRPMC in question. Their degradation mechanisms, effects on mechanical properties, exposure methods, and rationale for their inclusion in the study has been discussed below. Factors including acceleration, vibration, acoustic noise and mechanical shock have not been considered in secondary research as they have been deemed design parameters, investigated in preliminary design studies qualify materials for the specific application. Nevertheless, the synergistic effects of these factor with those investigated below are important to consider, particularly in regards to fatigue, and should be investigated further in future work. Factors which were reviewed but deemed unlikely to cause mechanical decrement are presented in Appendix A, Sections A.2.1 through A.2.4.

\section{High Temperature}

Temperature is the measure of the average kinetic energy of particles within a system. The higher the kinetic energy of a system, the higher the system's temperature. When discussing the effects of elevated temperatures on FRPMCs, the stability of

the polymer, and not the fibre is usually of greater concern as most fibrous materials (carbon, glass, boron, metals, etc.) show a greater resistance to degradation at elevated temperatures than their matrix polymer counterparts, with notable exception of polymer-based fibres, such as aramid and polyethylene.

Elevated temperatures can pose several problems for the polymer matrix of an FRPMC. One of the most prevalent issues is their ability to accelerate the effects of other degradation processes. For example, elevated temperatures can increase the chemical reaction rates by allowing a system to exceed the activation energy required 
for a reaction to proceed. While this issue can vary significantly based on the chemical species present in a polymer's working environment, an almost universal issue for polymers used in atmospheric conditions is thermal oxidation. While thermal oxidation can correspond to any chemically oxidative process, in this context, it refers to a polymer's thermally initiated oxidation reaction with ambient oxygen. The description of the mechanism of oxidation of hydrocarbons is based on the work of Bolland [23], and has been summarized by Hawkins [4] in Figure 1.4.

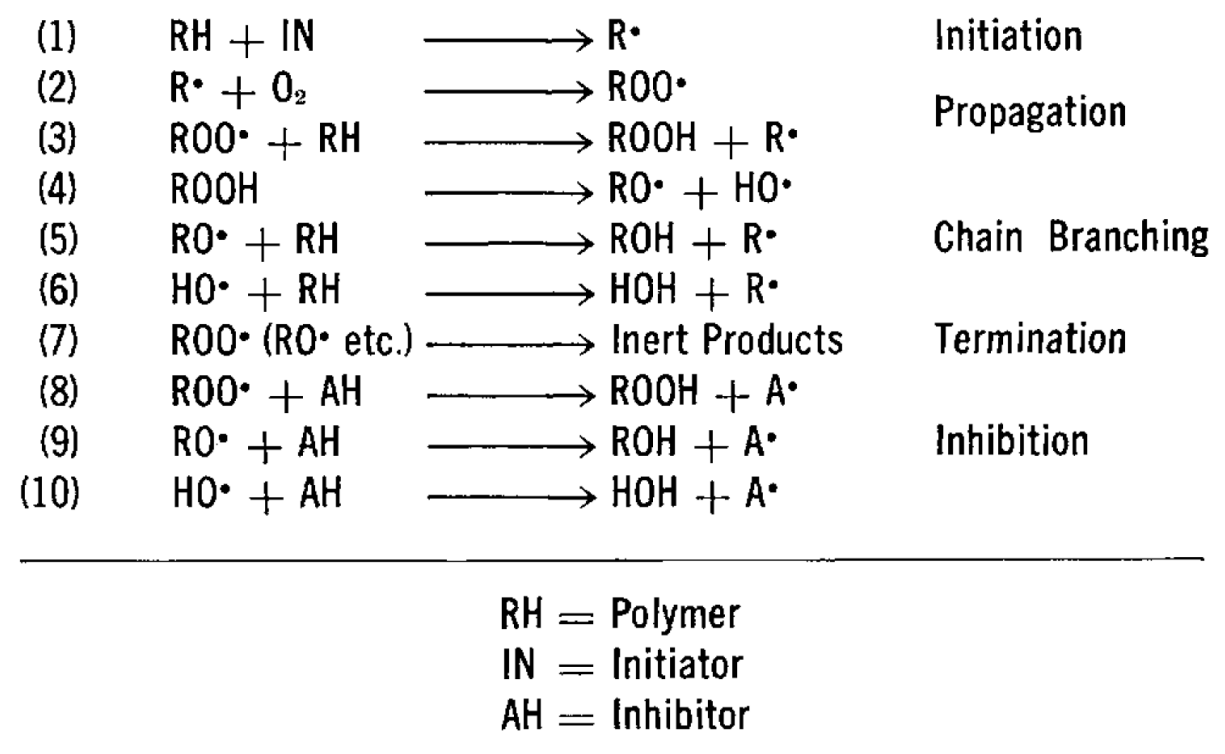

Figure 1.4: Kinetic scheme for hydrocarbon oxidation, adapted from [4].

In the case of thermal oxidation, initiation of free radical $(\mathrm{R} \bullet)$ formation is brought about by energy introduced through conductive, convective or radiative heat transfer. Free radicals are atoms or molecules which possess an unpaired electron, making them very chemically reactive. As a result, ambient oxygen willingly reacts with the free radicals produced, leading to further reaction with neighbouring polymer chains. While this cascade of reaction can bring about additional chain branching, chain scission is characteristically the dominant mechanism, resulting in loss of mechanical properties through chain shortening [24]. Thermal oxidation occurs through a wide 
range of temperatures, however higher temperatures allow for a greater rate of initiation by increasing the fraction of reactants which can exceed the activation energy barrier.

Physically, elevated temperatures can result in greater diffusivity of ambient fluids, accelerating chemical ingress or accelerate rates of deformation resulting from mechanical stresses (creep). Over a large temperature range, state changes in a material may also be a concern, as mechanical properties will be lost through transitions from solid to a liquid or gas. However, because polymers used in the aerospace industry tend to be thermosets, the high degree of crosslinking prevents transitions to a liquid state: as temperature increases to extreme levels, thermoset polymers will decompose through oxidation or thermal decomposition (breaking of intramolecular bonds) without melting (breaking of intermolecular bonds).

The temperature range in which FRPMC are stable varies depending on several factors including polymer composition, curing process and interface with the imbedded fibre. One metric which is used throughout industry to classify a polymer's thermal stability is its glass transition temperature, $T_{g}$. Glass transitions temperature is the temperature at which a polymer transitions from a brittle, glassy state to a ductile, rubbery state. It is important to note that the glass transition temperature does not represent a phase transition, such as crystallization or melt temperature, but instead shows a kinetic transition associated with a change in specific heat capacity. The glass transition temperature is a rate dependent parameter, that is, for a given material it can occur through a range of temperatures depending on the rate of heating or cooling. Above the glass transition temperature, a polymer becomes more ductile, resulting in a loss of stiffness and strength. Hence, it is extremely important that a polymer operates in the temperature range for which it was designed to avoid changes to mechanical properties. While not always the case, commonly, 
polymers which have higher glass transition temperatures have greater mechanical properties. Glass transition temperature is intimately related with polymer chain length and degree of crosslinking: the larger chains a polymer possesses and the greater the degree of crosslinking, the greater the glass transition temperature; this is a result of entanglement preventing relative chain motion. Thus, any degradation which results in chain shortening will result in a notable decrease in glass transition temperature. Use of glass transition as a quantitative metric for evaluating change in mechanical properties is ill advised as confounding mechanisms may impact the resulting properties [14], however, its consideration is critical for FRPMC component design.

Many studies have investigated the deteriorative effects of elevated temperatures on FRPMCs. Commonly, studies which look strictly to investigate the effects of thermal oxidation employ isothermal heating in an air-circulating oven [14, 25-28]. This process allows atmospheric oxygen to be present for reaction, allows an even heat distribution and prevents degradation due to confounding factors which may result from either conductive or radiative heat transfer. It may be noted that the vast majority of studies reviewed examining the impact of thermal oxidation did so at temperatures in excess of the cure temperature $[4,26,27,29,30]$. This was done in effort evaluate the mechanical performance of composites in extreme conditions as an academic exercise or to accelerate the oxidative ageing process, but may mask the true mechanism of thermal oxidation and degradation of in-service composites. While studies had varying composites, temperature levels and exposure durations, they consistently report a decrease in mechanical properties associated with prolonged exposure to elevated temperatures. In their study of the effects of thermal ageing on carbon fibre-reinforced bismalemide, Akay and Spratt reported that the tested composite exhibited mass loss 
and micro-crack formation which resulted in a notable decrease in mechanical properties [4]. While an increase in $T_{g}$ was noted, indicating an increase in the degree of crosslinking, up to a two-fold decrease in flexural and interlaminar shear strength was observed. Dao et al. investigated the Fourier transform infrared spectroscopy (FTIR) of an epoxy-carbon composite over one year of thermal ageing in air, at temperatures ranging from $70^{\circ} \mathrm{C}-200^{\circ} \mathrm{C}$ [31]. It was discovered that the surface chemistry varied depending on the ageing temperature. After 7500 hours at $70^{\circ} \mathrm{C}$, samples showed minimal changes to the chemistry of their bulk composite and showed surface changes primarily in concentration of hydroxyl and nitrile groups. However, at higher ageing temperatures, changes to nitrile group concentrations were noted deeper into the bulk for longer exposure durations and a greater variation of oxidation mechanisms were noted at the sample's surface.

Studies also reported on the synergistic effects of combined temperature and moisture exposure on composite degradation. These studies, referred to as hot/wet, hydrothermal (immersion) or hygrothermal (vapour) testing, attempted to identify the effects of elevated temperature on degradation resulting from exposure to moisture, rather than thermal oxidation, and were done almost exclusively at temperatures below $100^{\circ} \mathrm{C}$. This was done both, to simulate realistic environmental conditions and to prevent water evaporation. Hot/wet testing predominately involved composite samples immersed in a water bath at elevated temperatures for a set duration of time. Alternatively, some studies synergistically investigate the effects of temperature in conjunction with varying levels of humidity and UV radiation in what are called weathering studies. Reviewed hygrothermal and weathering studies showed that higher temperatures corresponded with greater rates of moisture ingress and corresponding degradation [32-34]. Chateauminois et al. analyzed the water diffusion and corresponding dynamical mechanical thermal analysis results of an epoxy-glass composite 
immersed in water at temperatures ranging from $30^{\circ} \mathrm{C}$ to $90^{\circ} \mathrm{C}[5]$. As demonstrated in Figure 1.5, all sample exhibited two stages of moisture diffusion: at short times a Fickian diffusion behaviour was observed and at longer times a non-Fickian behaviour was observed. An increase in capillary flow along the fibre direction during the nonFickian region was associated with disbonding between the matrix and fibres. At higher ageing temperatures the transition between Fickian and non-Fickian regions occurs after a shorter ageing period. This corresponded with a decrease in elastic modulus.

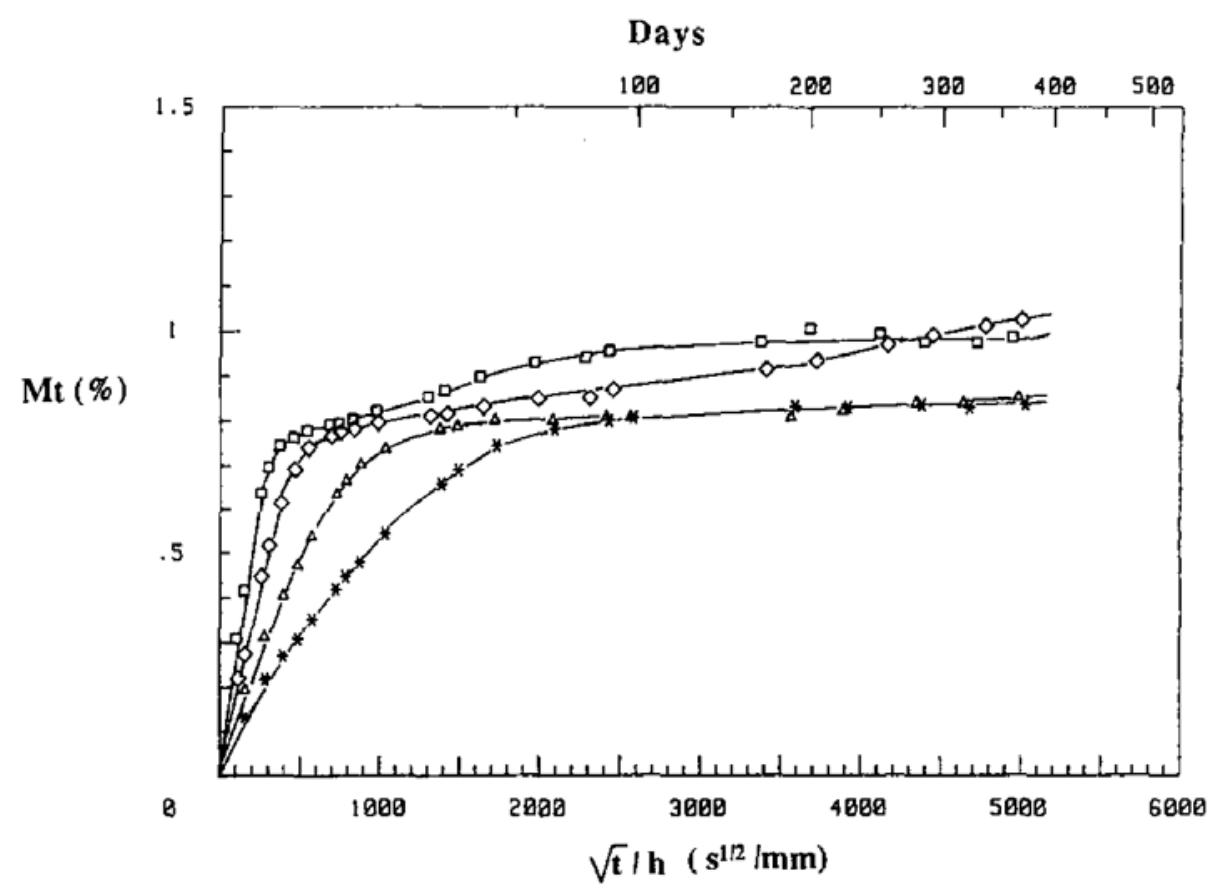

Figure 1.5: Change in composite mass as a function of time for epoxy-glass composite samples exposed to $30{ }^{\circ} \mathrm{C}(\star), 50^{\circ} \mathrm{C}(\triangle), 70^{\circ} \mathrm{C}(\diamond)$, and $90^{\circ} \mathrm{C}(\square)$ hydrothermal ageing, adapted from [5].

The glass transition temperature of carbon-epoxy composite under investigation in the present research is $151^{\circ} \mathrm{C}\left(304^{\circ} \mathrm{F}\right)$ [35]. Consultation with the industrial partner has revealed that the operating environment of the composite will not exceed the glass transition temperature, avoiding the associated loss in mechanical properties. However, it was identified that operating environment could reach up to $85^{\circ} \mathrm{C}\left(185^{\circ} \mathrm{F}\right)$. 
As identified by review, temperatures in this range have the potential to accelerate fluid ingress and induce surface degradation through thermal oxidation. For these reasons, investigation of degradation of FRPMCs due to and assisted by elevated temperature has been pursued further in primary research, both as means of accelerating degradation due to ambient chemical species and to replicate a worst-case, in-service environment.

\section{Water Exposure and Ingress}

The topic of exposure of FRPMC to water has seen a significant amount of research with water exposure documented to cause a decrease in mechanical properties. Water can come into contact with composite materials through a variety of forms such as bulk liquid, precipitation, fog or humidity with literature reporting varied conclusions on the relative severity of hydrothermal vs. hygrothermal ageing. For the purpose of this report each of these will be discussed as the same form of exposure, as they all contribute to the sample mechanism of degradation (neglecting synergistic effects such as precipitation erosion or elevated temperatures) although it is important to note that the route of exposure can have an effect on the rate and extent of degradation [36]. Moisture causes a reduction in the mechanical properties of FRPMC through irreversible chemical reactions with the polymer matrix [21,36]. Similar to elevated temperature and ultraviolet (UV) radiation exposure, moisture can bring about chain scissions leading to a lower average molecular weight of the polymer through chain shortening. These chain scissions are the result of oxidation and hydrolysis reaction $[37,38]$.

The severity of polymer degradation depends on two primary factors: the ability of 
water to achieve coordination with reaction sites of the polymer and the polymerwater affinity. Water's ability to achieve coordination with the polymer's reactions sites is a function of how effectively moisture can diffuse throughout the material. Diffusivity is a function of the material's structure; the greater the availability of water-molecule-sized holes, the more readily water will diffuse [39]. The availability of such holes is dependent on the material porosity, degree of crosslinking, curing process and matrix-fibre adhesion. If the matrix material shrinks during curing or there is poor adhesion between the matrix and fibre, capillaries can form around imbedded fibres, allowing for more effective diffusion [21]. Polymer-water affinity corresponds to the number of hydrogen bonding sites available. Not only are these sites locations of potentials chain scission reactions, but their presence dictates the overall hydrophilicity of the polymer. The greater concentration of these sites, the more hydrophilic the polymer and the more easily water molecules diffuse throughout the bulk composite. Such sites are characteristic of polar functional groups such as hydroxyls, carbonyls, carboxyls, or sulfhydryls. Hence, the greater the degree of functionalization with polar functional groups, the greater the polymer-water affinity. The availability of water soluble functional groups in a composite may also induce blister formation and delamination through osmotic pressure. In their work on blister formation on boat hulls, Rockett and Rose indicate that blister formation in glass reinforced polyester laminates can be brought about by osmotic pressure resulting from pockets of water soluble materials which induce water diffusion as a result of a concentration gradients [40].

To be able to predict the effects of moisture exposure on a composite material it is imperative to understand the mechanism and rate of moisture ingress. To make sense of this behaviour at a macroscopic scale, models may be develop based both on theoretical molecular diffusion and empirical evidence. Among the many proposed models, 
Fickian diffusions is by far the most commonly cited with many studies reporting moisture sorption in FRPMC composites at room temperature or slightly elevated temperatures demonstrating linear Fickian diffusion over relatively short periods of exposure [5,41-46]. However, over longer duration exposures, moisture sorption characteristics will often deviate from a linear Fickian model. In his extensive work on the effects of exposure to moisture on composite materials, Weitsman has generalized these deviations from Fickian into four groups as illustrated in Figure 1.6 [6]. In this figure, $L F$ designates a linear Fickian behaviour, $A$ shows behaviour which lacks weight-gain equilibrium, $B$ represents a two-stage diffusions characteristic and curves $C$ and $D$ shows a significant departure from Fickian behaviour, gaining and losing mass following linear sorption, respectively. Such deviations from Fickian behaviour have been attributed to simultaneous mechanism of laminate damage and diffusion; diffusion accompanying matrix deterioration, void formation and disbonding at the matrix-fibre interface $[5,46,47]$. A number of models have been proposed to characterize these anomalous sorption characteristics [48-50] however, their widespread adoption is hinder by the wide-array of composite-fluid systems present.

Mechanical properties of FRPMC exposed to moisture have exhibited various changes depending on degree of saturation and the property under investigation. Fracture toughness under mode I loading has been demonstrated to increase while decrease under mode II loading [51-53] the former predicted to be a result of increased plasticization [52], while the latter a result of degradation of the fibre-matrix interface [54]. This is supported by work noting a decrease to transverse tension and interlaminar shear [55]. Resistance to high strain rate impact events appears to increase due to room temperature moisture sorption as a result of the plasticization of the matrix polymer $[56,57]$ but may show a decrease at elevated temperatures. Most notably, moisture ingress in FRPMC has been shown to significantly affect fatigue response, 


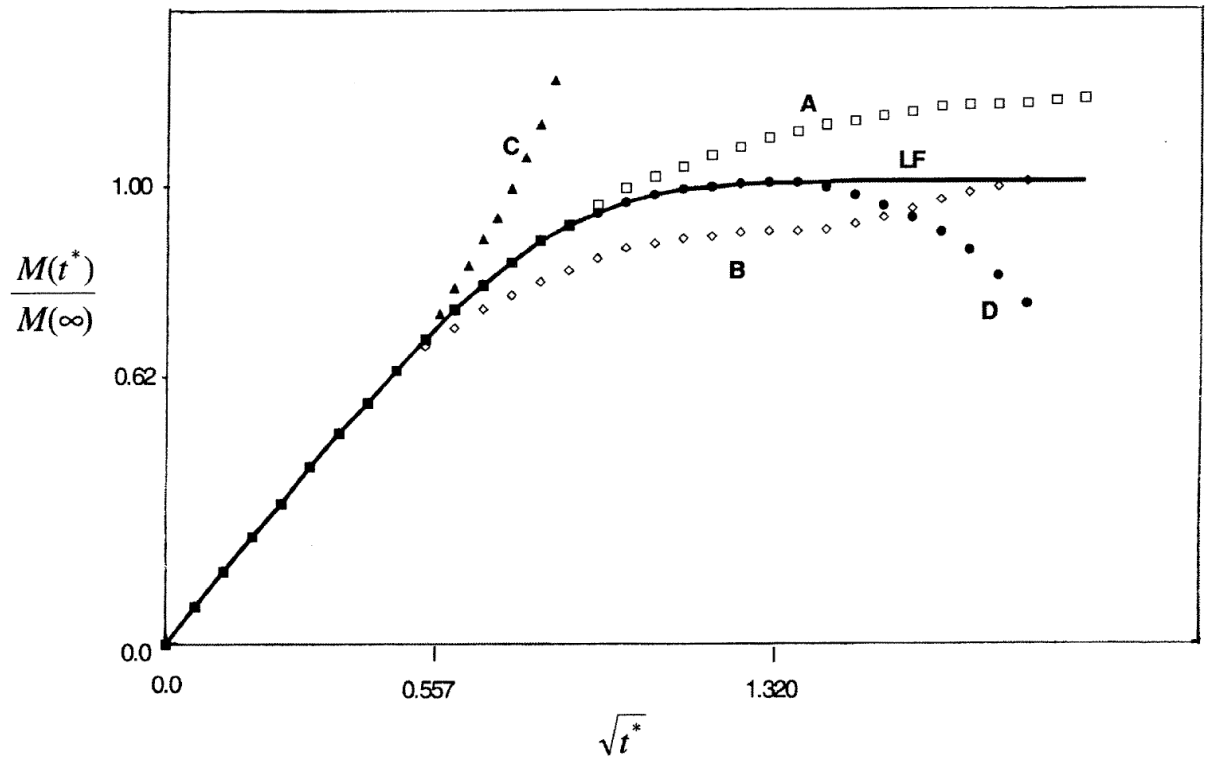

Figure 1.6: Non-Fickian mass-uptake behaviours over long duration exposure represented in terms of the square-root of non-dimensional time, adapted from [6].

with up to an $85 \%$ decrease in number of loading cycles before failure, which was observed in a vinyl ester-carbon composite subjected to complete immersion in sea water [58]. This effect was likely the result of extensive delamination caused by water trapped in transverse cracks during loading [59].

The time required to reach saturation and the percent weight change attained for a FRPMC system varies significantly depending on the composite thickness, polymer matrix, and fibre weight fraction. Work by Chatterjee and Gillespie Jr. on moisture absorption behaviour of an epoxy-glass system shows the moisture diffusion coefficient of epoxy-glass composites is higher than that of the neat constituent epoxy resin but that overall weight gain was lower due the fibre's poor ability to absorb moisture [37]. Their work, which observes the moisture absorption characteristics of three epoxies of varying degrees of crosslinking, monitored glass transition temperature as a means of evaluating degree of resin plasticization. What was demonstrated was glass transitions temperature decreased in association with the amount of water absorbed. However, 
upon drying, the samples reached glass transition temperatures higher than that of the pre-exposure control. FTIR demonstrated that this change corresponded with a segmented molecule leach out resulting in irreversible chemical changes to the matrix structure. Epoxies which possess a greater degree of crosslinking showed both a smaller diffusion coefficient and a smaller free volume after being dried.

An additional concern regarding moisture ingress in composites is their propensity to swell. Since absorption characteristics of fibre and matrix material differs, so too does their swelling coefficient hence, similar to the case of thermal expansion, moisture induced expansion causes strain resulting from material mismatch [60]. While these micro-mechanical strains are assumed not to effect material stiffness [60], they are believed to enhance crack initiation and propagation [61], thus lowering strength and enhancing moisture ingress to channel formation.

It is important to note that most of studies reviewed, evaluating the effect of moisture sorption, reported exposing samples to moisture through either complete immersion or exposure to water vapour in a weathering chamber. While these tests effectively show the effects of moisture sorption, they neglect to consider the moisture diffusion profile through the composite in a more realistic, single-side exposure. This point was addressed in Siriruck and Penumadu's study on the fatigue behaviour of vinyl estercarbon composite exposure to sea water. Their work demonstrated that single-side exposure resulted in $50 \%$ drop in fatigue cycles, compared to the $85 \%$ drop noted for a completely immersed sample [58]. Studies which have compared diffusion characteristics through the composite face (normal to fibre direction) and edge (parallel to fibre direction) have noted that the diffusion rate through the sample edge is significantly higher than through the sample's face $[62,63]$. This is likely as absorption normal to the fibre direction is impeded by a greater fibre surface area, and break down of the matrix-fibre interface can enhance diffusion through capillary action along the length 
of the fibre.

Consultation with the industrial partner has indicated that the composite material will be subjected to water exposure through direct contact with cleaning solutions and ocean water; fog and salt fog; and high humidity. Literature review has demonstrated that the sorption and degradation characteristics of moisture in carbon-epoxy composites has undergone previous investigation, and thus offers a strong point of comparison and methodology for testing alternative fluid exposure. The need for investigation of a more realistic, single-sided exposure test has been identified. For these reasons, investigation of FRPMCs degradation due to moisture ingress has been investigated in this research, as a means of comparison and validation for the moisture sorption characteristics specific to the selected carbon-epoxy composite.

\section{Chemical Exposure and Ingress}

While water is a chemical, chemical exposure described hereafter will discuss the exposure of FRPMCs to other chemical substances common to the aerospace industry. This distinction has been made due to the relative prevalence of potential routes of exposures to water compared to that of other chemicals and relative severity of degradation resulting from other chemicals compared to water. Additionally, discussion of chemical exposure will primarily focus on liquid chemicals. This is because, while composites are also exposed to solids and gases, the composite in question will have limited opportunities to encounter potentially harmful chemicals in either gaseous or solid states, as indicated by the industrial partner.

Although different in terms of severity and route of exposure, exposure to chemicals poses the same general mechanism of degradation as that of water: ingress chemicals react with either the polymer matrix or fibre material causing detrimental effects 
to the material's mechanical properties which can be accelerated through elevated temperatures. However, due to the varied nature of chemical substances, the specific chemical reaction which occur can be diverse in mechanism and effect. Some chemicals which FRPMCs used in maritime aircraft may be exposed to include brine (a salt solution in water); anti-icing fuel additives and de-icing fluids (propylene glycol or ethylene glycol based); aviation kerosene (a mixture of low molecular weight, aliphatic hydrocarbons); hydraulic fluid (varied composition with high molecular weight hydrocarbon base); and various cleaning solvents. While some studies have investigated the effects of specific chemical on constituent polymeric or fibre materials, relatively little research has concentrated on composite degradation resulting from exposure to chemicals common to the aerospace industry. Work by Yoshino et al. has investigated the mechanical performance of carbon-epoxy lap joints after exposure to some common aerospace chemicals [64]. Lap joint and neat adhesive samples were immersed in anti-icing additive, hydraulic fluid, water and aviation fuel for up to 10 and 555 days, respectively, at various temperatures. Sample were periodically removed from immersion, weighed and had their hardness measured. Through testing of the neat adhesive, it was demonstrated that anti-icing additive and hydraulic fluid induced a significantly higher mass uptake than that of water and aviation fuel at all temperatures, with aviation fuel showing minimal uptake at room temperature and water showing between a 5-7\% mass gain at all temperatures. At room temperature, samples immersed in water, aviation fuel and hydraulic fluid exhibited a minor increase in hardness, while the de-icing additive caused a major decrease in hardness. For samples which were tested at $85^{\circ} \mathrm{C}$ (aviation fuel exposure testing was not performed at elevated temperatures due to safety concerns), there was a negligible change in the hardness of the water immersed sample, however, the sample immersed in hydraulic fluid showed a significant drop in hardness and the degradation sustained by the de-icing additive sample was so great that accurate hardness values could not be 
obtained. Furthermore, work extending from this study analyzed the thermal properties, visual damage and attempted to model the diffusivity of the samples under the same exposures $[43,65]$. Samples conditioned in hydraulic fluid and de-icing additive showed visual degradation and a notable decrease in glass transition temperature. Results of the work attempting to fit gravimetric data to Langmuir and Fickian models showed that these models could be statistically valid for both adhesive and composite sample for some temperatures/chemical combinations, but demonstrated neither model successfully fit hydraulic fluid uptake and both the composite and adhesive samples exposed to anti-icing additive were shown to be more effectively represented by the Langmuir model. Work by Landry et al. looked at effects of immersion of carbon-epoxy samples in distilled water, hydraulic fluid and de-icing fluid on the delamination toughness [66]. Over one month of exposure delamination was increased up to $25 \%$ for water and de-icing fluid and up to $4 \%$ for hydraulic fluid. All fluid showed a significant increase in delamination growth rate although scanning electron microscopy (SEM) revealed minimal change to the observed fracture patterns. A study by d'Almeida compares the relative absorption characteristic of aramid-epoxy samples in distilled and salt water [67]. Interestingly, it was observed that salt water was absorbed at a slower rate than distilled water and as a result the interlaminar shear stress of the samples subjected to distilled water felt a more substantial decrease than that of the salt water samples. These results have also been demonstrated in other work [55].

This review has demonstrated the ability of chemical exposure to negatively impact mechanical properties. It has also found a gap in knowledge regarding the specific effects of aerospace chemicals on carbon-epoxy composites over long exposure duration and appropriate models for prediction of their diffusivity. For these reasons, investigation of degradation of FRPMCs due to chemical ingress has been pursued 
further in primary research.

\section{Low-Velocity Blunt Impact}

Consultation with the industrial partner has identified low-velocity blunt impact (LVBI) to be a common occurrence in the operating environment of the composite structure in question and thus an important consideration for secondary review. LVBI can occur on aircraft as a result of a variety of events. Externally, LVBI primarily occurs because of tool drop or impact during maintenance; bird impact during flight; stones thrown up by tires upon landing; hail; or from collisions with ground equipment, other aircraft or structures. Internally, LVBI is prominently the result of maltreatment during maintenance, although it can also result from improper crew and equipment motion. LVBI events are of particular concern for FRPMC composites as an event can induce barely visible impact damage (BVID) such as subsurface delamination and crack formation. BVID is often undetectable by conventional visual inspection techniques and can result in component failure through fatigue resulting from exposure to cyclical stresses.

A large amount of research has been devoted to LVBI, resulting BVID and its detection. In their work comparing the resulting impact damage on carbon-epoxy composites of varying stacking sequences, Morais et al. demonstrate that surface cracks resulting from LVBI follow the fibre orientation of the top ply and that cross-ply and non-symmetric laminates possess a better endurance to LVBI when compared to unidirectional reinforcements which provided "easy paths" for cracks to form [68]. Carbon-epoxy systems have been demonstrated to show two forms of failure events: delamination and back face matrix cracking and fibre failure, which can be measure with some propensity by evaluating the energy loss during impact [69] and can be 
predicted to occur for a given critical incident energy or for a given number of cycles of impact events below this energy $[70,71]$.

The review indicated that BVID in a laboratory environment is induced primarily through two methods: a drop tower apparatus for which impact force is a function of impact velocity, controlled through a dropped mass and the height from which it is dropped $[68,70,71]$ or some form of pendulum apparatus controlled through similar principles $[69,72]$.

Gaps identified in LVBI composite research are the scarcity of studies investigating synergistic effects of LVBI and other environmental factors on composite degradation and specific focus to edge (in-plane) impact opposed to surface (normal) impact, and resulting moisture diffusion. The former has been investigated in some regards, looking at impact in combination with hygrothermal conditioning $[56,73,74]$ however, these studies seek to determine hygrothermal effects on impact resistance and neglect reversed exposure; change in moisture diffusivity resulting from impact damage. A thermoplastic study of post impact moisture absorption in carbon fibre-PEEK laminates has however shown significant decrease to fatigue life [75]. Edge impact has been investigated to some degree as well [76-78], yet minimal research has focused its analysis on subsequent moisture uptake. While impact damage has not been investigated further in this work due to time constraints, it has been recommended that future work investigate the synergistic contributions of either surface or edge impact damage with hydrothermal and chemical exposures for an epoxy-carbon composite. 


\subsubsection{Evaluation Techniques}

While the methods of exposure varied depending on the factor under investigation, a large number of commonalties can be drawn between experiments and how they evaluated the effects environmental factors had on the composites. Evaluation methods reviewed can be divided into either destructive testing or non-destructive testing. The following describes both categories and some methods commonly identified through literature review. All studies reviewed took a material-focused, non-structure specific, coupon-level testing approach, as the material properties and not the structural properties were of primary interest.

\section{Destructive Testing}

Destructive testing is the most effective, most direct method of obtaining a quantitative metric by which to evaluate changes to material properties. As the name suggests, the process of measuring a material though this means is destructive, significantly impairing or preventing the material from being able resist subsequently applied forces. This makes them impractical for evaluation of in-service components, for which a loss of mechanical properties would require the component be removed from service. Material testing methods of this nature are standardized by a number

of governing bodies such as the American Society of Testing and Material (ASTM), the International Organization for Standardization (ISO) and the British Standards Institution (BSI). Methods referenced in this text will be that of ASTM, however, many similar, if not identical, testing methods have been standardized through other organizations. Destructive tests discovered through review fell primarily into two categories: mechanical, which examined a material's stress-strain characteristics in response to loading, and thermal which examined a variety of properties in response 
to changing temperature. In some instances, mechanical and thermal tests were combined to investigate stress-strain characteristics under varying temperature.

The review demonstrated that a large variety of mechanical destructive tests are employed to evaluate the effects of environmental exposure. Predominantly, tests are selected which illustrate the matrix-dominant properties as environmental factors have been shown to more severely degrade the polymer matrix. Such tests include evaluation of flexural strength and modulus; compressive strength; interlaminar shear strength and modulus; short beam strength (SBS); and interlaminar toughness. Tensile testing is also commonly used to evaluate environmental degradation; however tensile properties are only considered to be matrix dominant if measured in the transverse direction or off axis (i.e. not parallel to fibre direction) in a unidirectional composite. Longitudinal tensile strength was sometimes evaluated however, this is likely due to ease of testing. Hardness and wear tests were sometimes implemented as well, although these methods only relay information about degradation at a material's surface. While literature rarely reveals the rationale behind the selection of a specific destructive testing technique beyond its ability to reveal changes to matrix or fibre dominant properties, it may be presumed that the selection is dictated based on anticipated stress exposure for an industrial application, availability of testing equipment, popularity of methods as revealed by literature, the ease of implementation or repeatability. Both static and dynamic mechanical tests were implemented to evaluate environmental exposure effects. While static testing was predominantly used likely due to its relative convenience and its effectiveness as a means of comparison, fatigue tests offer a more direct insight into the effect of exposure on composite service life.

Thermal analysis reviewed were primarily thermo-gravimetric analysis (TGA), differential scanning calorimetry (DSC) and dynamic mechanical analysis (DMA). TGA involves monitoring the mass of a minute sample as its temperature is increased at 
a steady rate. Temperature increase can be continuous or stepwise with isothermal holds. TGA is a useful technique to evaluate a number of factors related to composition including the weight percentage of additives, such as fillers and plasticizers, and can provide information about the degree of cure through indication of residual solvent and low molecular weight content as a function of temperature. For evaluation of the effects of environmental degradation, TGA is valuable for determining residual moisture content, when investigating effects of moisture ingress; the polymer's oxidative stability, when investigating the effects of elevated temperatures; and changes to a composite's decomposition temperature which can be an indication of irreversible chemical changes.

DSC examines changes in heat flux into a sample compared to a reference. Thermocouples in contact with the sample and reference material measure the local changes in heat as both are subjected to identical heating and cooling profiles. The presence of exothermic or endothermic heat flows is a key indication of a state or kinetic change of the material. Hence, DSC is useful for detection of changes to glass transition and phase transitions such as melting and crystallization.

Both TGA and DSC techniques can be performed in either an inert gas environment or an oxygen-present environment, depending on the degradation mechanism of interest. TGA and DSC are both useful tools for assessing the level of deterioration of polymer matrix composites. However, neither technique reveals quantitative changes to mechanical properties and as such cannot be used as the sole indication of an exposure's ability to degrade a composite. Both should be used only as supplemental tools to qualitatively indicate degradation and the requirement for further mechanical testing. 
DMA reveals a material's response to an oscillatory stress-strain and how that response changes with temperature and frequency. The mode by which the oscillation is applied (i.e. tension, compression, etc.) and the heating rates applied may vary depending on test and the parameter of interest. DMA can be used to determine a materials storage modulus, loss modulus and glass transition temperature. While DSC is more widely used for determination of glass transition temperature, DMA is consider to be a more sensitive detection method and is effective for determination of $\mathrm{Tg}$ for materials which are highly crosslinked, such as epoxies.

\section{Laboratory Nondestructive Testing}

A subset of evaluation techniques reviewed may be classified as non-destructive in nature but are impractical for use on in-service aircraft, unlike the techniques described in Section 1.3.2. These tests do not provide information about degradation suffered through a destructive method and are not practical for use in the field due to the requirement of specialized equipment, the size of test specimens, the requirement of a controlled testing environment, or a combination of these factors. Hence, these tests are generally performed in a laboratory setting.

The most common technique used under this category is the monitoring of a sample's mass through periodic measurements on a scale or microbalance. By monitoring a composite's mass over the duration of exposure, researchers can evaluate a composite's propensity to gain mass which can reveal moisture ingress or chemical reactions taking place with ambient species, both of which can signify a loss or gain of mechanical properties. Complementary, dimension and volume changes can be evaluated through use of a micrometer. Noted expansion or contraction of a sample can arise through chemical exposure or changes in temperature, imposing micro-stresses on the material 
and potentially leading to degradation.

Microscopy is often used to visualize the effects of degradation which are not visible to the human eye. In the context of environmental degradation of composite materials, microscopy is particularly important for revealing microscopic degradation modes such as cracks, delamination, disbonding, pore formation or other forms of surface aberrations $[25,29,32,33,37,79,80]$. Microscopy is also useful for analyzing changes to a composite's failure mechanisms brought about by environmental exposure. While there are a variety of microscopy techniques which can be implemented, some of the most common to this area of research are optical microscopy and SEM.

Optical microscopy is one of the most accessible and economical forms of microscopy. Optical microscopy functions by focusing visible light which is either transmitted through or reflected from a sample of interest using a series of lens. For the reasons previously mentioned, as well as the requirement for minimal sample preparation time and ease of use, optical microscopy is one of the most widely used forms of microscopy. However, optical microscopy has a resolution limited by the wavelength of visual light and the numerical aperture of transmission medium which makes visualization of sub-micron sized features impractical. For smaller feature sizes, SEM is conventionally used. SEM relays information of a structure's surface through the reflection, deflection and absorption of electrons. Electrons which are emitted from a source are focused through a sequence of electromagnetic lenses such that the focal point lies at the sample surface. In its traditional mode of operation, the beam will be raster-scanned over the sample's surface and repulsive interaction will cause emitted electrons to be scattered back away from the sample. Detectors will then pick up the scattered electrons and a greyscale image may be produced based on the relative areas of high and low election concentration received. SEM is a versatile technique which is able to detect nanoscale surface defects. Additionally, x-ray photoelectron 
spectroscopy (XPS), a technique which is an extension of SEM, allows for detection of elemental composition in a sample surface which can be useful for analyzing the absorption of a chemical species. Unfortunately, SEM can sometimes be less accessible due to its cost, restrictive sample size and its requirement for samples to possess an electrically conductive surface (this can be achieved for non-conductive samples through application of a metallic coating).

Another imaging technique encountered through literature for composite evaluation was x-ray micro-computed tomography $(\mu$-CT) [58,81] . $\mu$-CT allows for the generation of a three-dimensional model of the interior of a solid object. It does this by generating an assemblage of two-dimensional images which are then compiled in to a digital model. The two-dimensional images, or slices of the object. are captured by a camera capable of detecting x-rays emitted from an x-ray source, through the sample. Variations in pixel greyscale values of obtained slices are a result of the xray interaction, or lack there of, with the sample. $\mu$-CT has the ability to generate high-resolution images and benefits from being able to obtain internal cross-sections without access to a visible surface, usually requiring sawing which has the potential to damage the exposed surface.

A tool used in literature to chemically analyze FRPMC samples is FTIR [27,32,37,82]. As a spectroscopy technique, FTIR looks at how radiation interacts with matter; infrared light (possessing wavelengths between $750 \mathrm{~nm}$ and $1 \mathrm{~mm}$ ) are used to determine the types and relative amount of certain bond types in a material. The technique works by first projecting a range of infrared light on to a sample. If a photon possess the same vibrational frequency as that of a molecular bond it comes in contact with it will be absorbed by that molecule. Results are obtained as intensity as a function of wavenumber. FTIR is an excellent technique for detection of sustained degradation. By comparing the FTIR spectrum of a composite exposed to environmental factors 
with that of an unexposed control it is possible to observe resulting bond breakage and bond formation, offering insight the chemical mechanisms of degradation and degradation by-products. FTIR is typically used as a qualitative metric due the complex and difficult to analyze nature of the resulting spectrum. FTIR is sensitive to the secondary structure within a bond's local environment. Hence, FTIR will often produce broad, overlapping peaks making it difficult to quantitatively concluded bond concentrations.

The last laboratory based nondestructive method which was noted with some consistency through review was nano-indentation and nano-scratch techniques [79, 83, 84]. While both techniques may be deemed destructive in nature, the nanometer length scale over which damage is induced results in minimal decrement to a structure's macroscopically observed mechanical properties. Nano-indentation and nano-scratch techniques have come about to bridge the gap between FTIR spectroscopy and bulk, destructive testing methods, in order to determine the mechanical properties of the fibre-matrix interphase region [79]. Similar to Vickers or Knoop hardness tests, nanoindentation uses a pyramidal impression tip to induce a deformation in the material's surface. Based on the loading required to generate an indentation the hardness and reduced elastic modulus of the materials may be determined [83]. This technique can be used to compare these properties for various locations in a sample (i.e. for the matrix, fibre and interphase region) and can be extended to compare materials subjected to various exposes. Similarly, the nano-scratch test uses a pyramidal tip to probe a sample, however, it moves the tip tangentially across the sample surface with a fixed normal force. This process allows the test to detect both the coefficient of friction, and the size of analyzed regions, by evaluating the change in coefficient of friction over the measurement length [79]. Both nano-indentation and nano-scratch tests offer key information about the local characteristic of interphase regions at select 
points of a cross-section. However, due to their inherently small scale of measurement, they do not have the ability detect loss in mechanical properties sustained through macroscopic degradation modes, such as delamination and crack formation.

\subsubsection{Review Summary}

Through the literature review and consultation with the industrial partner, five principle forms of environmental exposure have been identified which may cause degradation to an epoxy-carbon based, FRPMC composite intended for use in the interior of a maritime-based, military aircraft: elevated temperature, water, chemical, salt fog and low-velocity blunt impact.

Degradation of FRPMCs due to elevated temperature may be attributed to thermal oxidation and thermal decomposition. Elevating temperatures is extensively used to accelerate the degradation of FRPMC as a result of ageing processes, i.e. reactions with ambient chemical species resulting in chain scission of the polymer matrix. Elevated temperatures are also used to evaluate FRPMC creep mechanics resulting from increased chain mobility. Exposure methods implemented are typically convective in nature for studies solely investigating thermal degradation but can be conductive when evaluating the synergistic effects of fluid exposure. While temperature effects of FRPMCs have been extensively studies in academia and industry in order to evaluate the optimal conditions of operation associated with specific, desired mechanical properties, little research has evaluated the synergistic effects of thermal oxidation, in conjunction with other environmental factors on composite degradation over protracted durations.

Degradation of FRPMCs due to water, chemicals and salt fog may be attributed to reactions with the polymer matrix resulting in chains scission. This can lead to 
weakening of the FRPMC through a combination of degradation of the polymer matrix itself and degradation of the polymer-fibre interface. This process is based on the polymer-chemical reactivity and the diffusivity of the chemical through the polymer matrix, the latter being further categorized by the polymer porosity and the relative polarity between chemical and polymer. Water absorption has been fit to Fickian as wells as various, anomalous diffusion models depending on the composite investigated. Accelerated ageing is effectively achieved by either increasing chemical concentration or increasing ambient temperatures, the former increasing the availability of reactive species and the latter both improving fluid diffusivity and increasing the number of species which may surpass the reaction activation energy. Exposure to water has been performed through either sample immersion or exposure to vapour in a weathering chamber and exposure to other chemicals has primarily been performed through sample immersion. While moisture ingress of FRPMC has been the focus of a significant amount of research, few studies have investigated the diffusivity of, and effects of degradation caused by common aerospace fluids; knowledge which may be practical for component life prediction and inspection frequency. Moreover, relatively little research has focused on a more realistic, single-surface chemical exposure which would allow for distinction between edge and surface ingress in a practical approach.

Degradation of FRPMCs due to LVBI is physical in nature, with an impact event, or repeated impact events causing cracks or delamination. LVBI exposure is achieved through controlling the momentum of a mass which collides with the composite sample in either a drop tower or pendulum apparatus; the degree of BVID is then evaluated through the energy loss during impact, visual inspection, or some form of NDI technique such as ultrasonic assessment. Accelerated ageing is the context of LVBI is achievable by increasing impact force for a single event or increasing event frequency. BVID has seen a fair amount of research, both in terms of its effect on 
material strength and its detection. Research has looked at synergistic hygrothermal contributions as well as damage sustained from impact to the surface, edge and near edge of a laminate. However, minimal research has sought to determine a correlation between LVBI, fluid ingress from subsequent exposure and the resulting degradation. Furthermore, no research was discovered which focused on aerospace chemical ingress in FRPMC post impact.

The degree of degradation and its effect on mechanical properties were evaluated both destructively and non-destructively. Destructive techniques offered a quantitative evaluation of a material's mechanical properties, providing a direct indication of an exposure's capacity to reduce strength and stiffness. Destructive techniques primarily focused on matrix-dominant properties with use of a universal testing machine with tensile, compressive, interlaminar shear, flexural, interlaminar toughness and SBS testing being some of the most common metrics evaluated. Thermal techniques used to evaluate thermal responses linked to changes in mechanical properties included TGA, DSC and DMA. Popular laboratory-based non-destructive testing included various forms of microscopy, mass analysis, FTIR, hardness testing, and nano-indentation. 


\section{Chapter 2}

\section{Methodology}

This chapter discusses the methodologies employed to prepare and run the experiments proposed. Following experiment proposal, areas discussed include flexural and short beam strength (SBS) testing; flexural fixture design, fabrication and validation; a description of the selected laminate material, its layups and curing procedures; coupon preparation and conditioning; conditioning test fixture design, materials selection and evaluation; assessment of moisture uptake and metrology; and discussion of supplementary evaluation techniques used to qualify degradation.

\subsection{Experiment Proposal}

Based on the literature review provided, material availability, equipment accessibility, monetary and temporal constraints, a sequence of experimental campaigns has been proposed to address identified gaps in knowledge relevant to the degradation of aerospace, FRPMCs.

The first test campaign focused on chemical induced degradation achieved through 
pragmatic exposure. The primary objective of this campaign were to:

- Determine the relative effect of exposure to chemicals common to military aircraft on the mechanical properties of a monolithic composite laminate

- Determine the relative diffusivities of chemicals common to military aircraft with regards to the monolithic composite laminate and attempt to fit the observed trends to models proposed in literature

- Devise an association between degradation observed and fluid uptake

To achieve these objectives a two-factor, factorial design was proposed. The two factors consider were chemical type and exposure duration, each of which was tested at six levels with three replications (i.e. a $(3) 6^{2}$ factorial design) with flexural strength, flexural modulus, mass and volume as associated response variables. Chemicals chosen were selected for their known use in aerospace. Chemicals included deionized water (hereafter referred to as DI water), artificial ocean water (hereafter referred to as ocean water), Royco 782 synthetic hydraulic fluid (hereafter referred to as hydraulic fluid), Aeroshell turbine oil 390 (hereafter referred to as turbine oil) and reagent-grade ethylene glycol - a prevalent component in deicing fluid - in addition to a duration specific control (air). Ocean water was synthesized using Instant Ocean, a commercially available salt mixture intend for salt water aquariums which contains salt concentrations representative of ocean water. Samples were to be exposed to their respective fluid on a single surface, for one of six prescribed durations; 0 hours, 24 hours, 168 hours (one week), 720 hours (one month), 1440 hours (two months) and 2160 hours (three months). To accelerate the rate of moisture uptake, and to simulate a worst-case exposure condition in accordance with the United States of America military standard, MIL-STD-810G 5, method 501.5 [22], samples were aged 
at elevated temperatures $\left(85^{\circ} \mathrm{C}\right)$ in a convection oven. Upon removal from condition, samples were weighed and measure in accordance with the standard operating procedure $(\mathrm{SOP})$ presented in Section B.1 of Appendix B prior to being subjected to flexural testing as presented in Section B.3. In conjunction, the longest duration samples had mass measurements taken continuously, over a standard interval (three days) in order to evaluate fluid uptake. This, however was halted prematurely for reasons discussed in Section 4.3. Secondary evaluation, used to characterize degradation, included optical microscopy and micro-computed tomography.

The second test campaign looked to validate the relative chemical dependent-effects of the first campaign through complementary SBS testing following sample immersion. In this manner, the second campaign allowed comparison of the effects on flexural properties to shear properties and allowed comparison of sorptions rates for singlesided exposure vs. full immersion. Following a similar $(3) 6^{2}$ factorial design, the second test campaign observed effects of the same chemicals on the SBS strength, mass and volume response variables, but incorporated floating point evaluation intervals, allowing for meaningful evaluation of SBS in relation to the anticipatively higher fluid absorption rate. Similar to the first campaign, metrology of the longest duration samples was performed over a standard interval (three days) in order to evaluate fluid uptake. Secondary evaluation included DSC and TGA thermal techniques. In addition, hardness testing was evaluated as a possible, minimally invasive inspection technique to quantify fluid-induced degradation.

Both testing campaigns were accompanied by statistical analysis of the obtained data to ensure significant results and validity of conclusions. 


\section{$2.2 \quad$ Flexural Testing}

As demonstrated through literature review, mechanical degradation resulting from chemical ingress is matrix-dominant. As such, an evaluation technique which characterizes matrix-dominant mechanical properties is essential to accurately portray the severity of exposure. This is assuming the composite structure will be subjected to a variety of forces and that decrement to any structural property is of concern; for example, evaluation of change to the compression strength of a cable held in tension may not provide useful information. Hence, flexural strength and SBS tests were selected as both are matrix-dominant, requiring load transfer between the fibres through the matrix in order to resist an applied force. Beneficially, flexural and SBS tests require a relatively simplistic sample geometry and test setup compared to other static, matrix-dominant evaluation techniques such as compression and fracture toughness. Additionally, as both flexural and SBS tests are similar in execution they have the capability to be performed using the same test fixture given minimal fixture versatilely (e.g. roller diameter, span length and accepted compressive force).

Flexural testing provides the stress-strain response of a material in flexure from which a material's flexural strength and flexural modulus of elasticity may be determined. From Euler-Bernoulli beam theory, a simply supported beam which possesses a symmetric cross-section will be subjected to stress, $\sigma$, given by

$$
\sigma=\frac{M x}{I}
$$

where $M$ is bending moment, $x$ is the distance from the neutral plane to the point of interest and $I$ is the second moment of area. For a simply supported cuboidal beam with force applied at the midpoint of its span, the bending moment is given as 
$\frac{P L}{4}$ where $P$ is the force applied at mid-span and $L$ is the length of the beam. As the outer surfaces at midspan is subjected to maximal stress with stress decreasing linearly towards the neutral axis, failure will first occur at either the top or bottom surface. Hence, the point of interest may be described by $\frac{h}{2}$, where $h$ is beam thickness. Knowing that the second moment of area of a cuboidal cross-section with respect to the width-wise axis is $\frac{b h^{2}}{12}$, where $b$ is the width of the beam, the equation for flexural stress on a cuboidal beam may be expressed as

$$
\sigma=\frac{3 P L}{2 b h^{2}}
$$

Hence, maximum flexural strength is the maximum stress a beam can withstand prior to yielding or fracture. In order to determine flexural modulus, the strain on the beam must be determined. For a beam of length, $L$, subjected to deflection, $\delta$, strain, $\varepsilon$, may be calculated as

$$
\varepsilon=\frac{6 \delta h}{L^{2}}
$$

By knowing sample dimensions, applied loading and resulting deflection, the flexural modulus of elasticity, E, may be determined from the slope of the elastic region of the stress-strain curve such that

$$
E=\frac{\Delta \sigma}{\Delta \varepsilon}
$$

where $\Delta \sigma$ and $\Delta \varepsilon$ are the change in stress and strain, respectively. Importantly, these equations are based on homogeneous beam theory. As FRPMCs are not homogeneous 
in nature the resulting strength and modulus values achieved are apparent values; estimations based on an a equavalient geometry, homogeneous beam. Asymmetries in stacking sequence can shift the neutral axis from the center plane of the overall geometry, confounding results. Hence, while asymmetric laminates may be tested, it is important to keep in mind flexural testing can only provide and estimation of true flexural properties. Furthermore, local variations in laminates brought about by inconsistencies during layup and curing procedures can have significant ramifications on materials properties from layer-to-layer and from sample-to-sample . It is imperative that coupons are derived from laminates which have been subjected to stringent fabrication and inspection processes.

There are a number of standards available which describe methods for testing the flexural properties of materials. For polymers, the most common include ASTM D790 [85], intended for homogeneous polymers, and ASTM D7264 [86], intended for polymer matrix composites. The primary difference between D790 and D7264 is the specified aspect ratio of sample's support-span-length to specimen-thickness; D790 specifies a 16:1 ratio while D7264 specifies 32:1. This minute variation is important to ensure the failure in higher stiffness composite materials is a result of flexural forces (tension or compression at the outer-most surfaces) rather than shear. For this reason, ASTM D7264 was selected for experimentation.

Testing standards provide methods for both three-point and four-point bending tests, where four-point tests position two loading noses at either one-quarter $[86,87]$ or onethird [87] span lengths from the lower supports. In this manner, four-point bending ensures the areas between loading noses is subjected to an equivalent maximum stress, rather than only achieving maximum stress at a cross-section directly below a central loading nose. For materials with inherent, periodic defects - such as cracks, channels or voids - four-point loading is superior as it produces a lower distribution of 
maximum flexural strength. This is because the area between noses has a higher probability of containing a defect than the cross-section directly below a central loading nose. However, as maximum deflection still occurs at midspan due to four-point bending, it necessitates the use of a transducer, deflectometer or some form of optical technique to accurately measure deflection. Hence, due to its simplicity and the availability of equipment, three-point bend testing was selected for this experiment.

\subsubsection{SBS Testing}

SBS testing, as described by ASTM D2344, [88], allows for determination of a shortbeam strength, a shear dominant parameter. SBS testing cannot evaluate pure shear stress due to the test's nature; three-point bending induces a complex-stress state of tension, compression and shear which is further complicated by local forces at the supports and loading nose $[89,90]$. However, the SBS test, if performed correctly, can evaluate a material strength associated with interlaminar shear do to high shear strength at the midplane. SBS testing described in ASTM D2344, [88], uses a supportspan-length to specimen-thickness ratio of 6:1 to minimize tensile and compressive bending stresses. With knowledge of the force applied and sample dimensions, shortbeam strength, $F^{S B S}$, can be calculated as

$$
F^{S B S}=\frac{3 P}{4 b h}
$$

While no standard test method is capable of creating a pure shear stress condition [91], standards exist which provide an approximation of pure, in-plane shear [91, 92] however, these tests require both complex test fixtures and sample geometries making preparation and testing more time consuming and costly. Conversely, SBS testing 
utilizes a simple sample geometry and requires minimal material, making it ideal for large-scale evaluations comparing the relative effects of environmental exposure. Additionally, interlaminar shear induced as described by ASTM D2344 represents a more realistic failure mode for the structure under investigation.

\subsubsection{Fixture Fabrication}

In order to perform testing as prescribed by ASTM D7264 [86] and ASTM D2344 [88] an appropriate test fixture was required. A simple cost-benefit analysis revealed that the most economical means of acquiring a flexural test fixture would be to manufacture one using materials and facilities available through the Carleton University.

A flexural test fixture consists of two supports and one, or two, loading nose or noses; the two supports, simply support the sample during testing through two cylindrical contact surfaces and the loading nose or noses apply a force to the sample through a similar contact surface at either midspan or at defined fractions of span length, depending on whether a three or four-point bending configuration, respectively, is used. The supports and nose structures must be adjustable with respect to one another such that their position can be changed to match sample dimensions and must possess some form of adapters which allow them to be secured and aligned in a universal testing machine (UTM). Support structures must possess smooth surfaces, free of defects, to prevent inconsistent, unrepresentative failure modes. Additionally, the test fixture cannot deform under the loading require to cause the sample to fail.

A conceptual flexural fixture was drafted and modeled using AutoCAD design software. Figure 2.1 shows the completed flexural fixture with axial extensometer secured, in a SBS configuration, mounted in the UTM. Orthographic projection drawings of the fixture's constituent components have been presented in Appendix C. 
This simple design allowed the support and nose structure to be interchangeable, an adjustable support span and functionality in both three-point and four-point bend configurations. Base components and support anvils consisted of 7050 aluminum and were machined with a three-axis, computer numerical control (CNC) milling machine. Cylindrical contact surfaces (rollers) and roller pins were cut from hardened M2 tool steel rods. Lastly, 4140 steel male adapter rods were machined to size using a lathe.

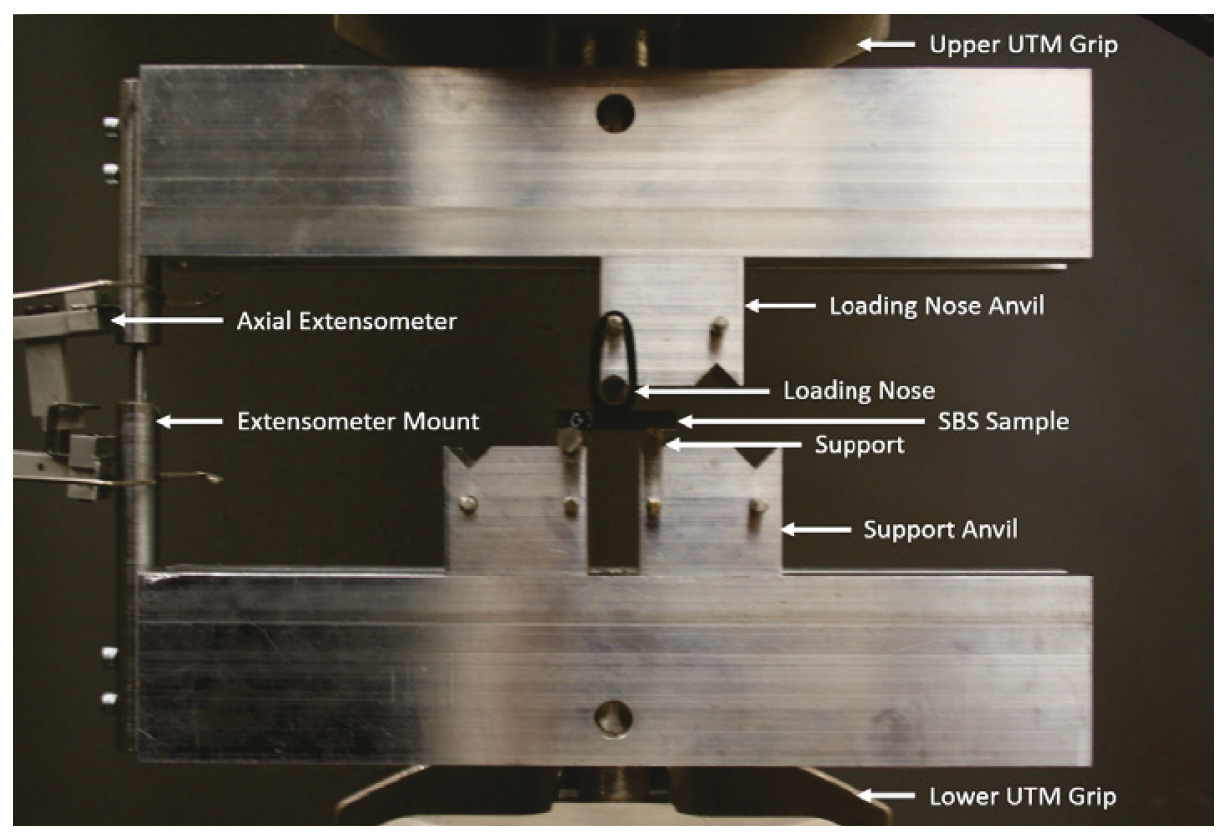

Figure 2.1: Flexural fixture assembly in a SBS configuration.

In this assembly, the rollers are seated in v-notches in the support anvils, the anvils are held to the base component with socket cap screws threaded into a t-nut seated in the base's t-channel and the male adapter is connected to the base component with a socket cap screw, screwed parallel to the rod's axis.

In order to accurately measure sample deflection, a mount was machined from 4140 steel rod which was fastened to both halves of the flexural fixture, allowing an MTS 634.12 axial extensometer to be secured to both components. While this means of measuring strain circumvents compliance in the crosshead, it is not ideal as it does 
not take into consideration rotation of the specimen about the loading nose and supports, nor account for compliance in the loading nose. Ideally, to address these issues, a transducer should be used to measure deflection through contact with the middle of the support span, although this apparatus was unavailable for the proposed testing.

\subsubsection{Fixture Validation}

In order to determine the precision, accuracy and reliability of the fabricated test fixture, a sequence of flexural tests were performed. These tests were carried out on 6061-T6, T651 aluminum samples with dimensions adhering to ASTM D790 standard [85]. As metals are traditionally homogeneous and linear-elastic until yield, they do not have a standard flexural test method as, assuming an ideal sample free of defects, flexural properties are equivalent to tensile properties - ultimate tensile loading induced during flexure at the outer surfaces is equivalent to that observed in a traditional longitudinal tensile test. Thus, for the purpose of this test, flexural values were compared to literature tensile values: a yield strength of $275 \mathrm{MPa}$ and modulus of elasticity of $69 \mathrm{GPa}$ [93]. Three groups of samples were tested: group one (samples 1 - 6) were cut with a band saw, group two (samples 7 - 9) were cut with a diamond saw blade and group three (samples 10 - 12) were prepared identically to group two but tested on a different day. Segregating samples in this manner allowed for an investigation of the effects of varied sample preparation techniques and ascertained repeatability of day-to-day measurements variability, the result of setting up the fixture each day. Figure 2.2 displays the toe-compensated stress-strain curve obtained for the 6061-T6 standard flexural test. 


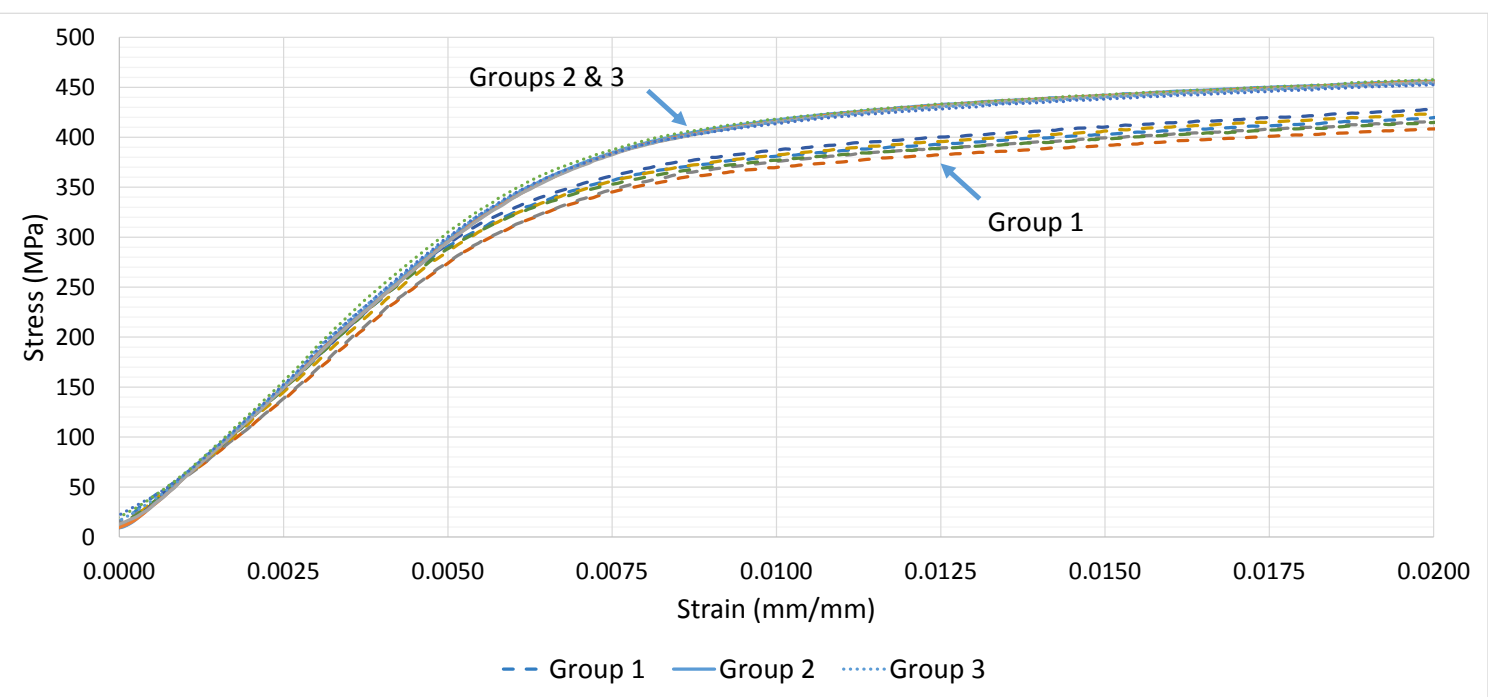

Figure 2.2: Stress-strain relationship for 6061-T6, T651 aluminum flexural test.

As observed, the curves are smooth with clear regions of elastic and plastic deformation, without any discontinuities, peaks or spikes which may allude to compliance issues, impeded motion of the fixture or failure of the fixture's constituent components. While all three test groups demonstrated similar moduli, there is a clear dissimilarity between the yield strengths of group one and groups two-three samples. Table 2.1 presents the flexural data as it pertains to each group: strength values represent the offset yield strength at $0.2 \%$ plastic strain and the moduli are presented as the slope from 0.001 to 0.006 stain.

Table 2.1: Offset yield strength and moduli values averaged over each 6061-T6, T561 test group.

\begin{tabular}{ccccccc}
\hline \multirow{2}{*}{$\begin{array}{c}c \\
\text { Group }\end{array}$} & \multicolumn{2}{c}{$\mathbf{2}$} & \multicolumn{2}{c}{$\mathbf{3}$} \\
Property & Modulus (GPa) & Strength (MPa) & Modulus (GPa) & Strength (MPa) & Modulus (GPa) & Strength (MPa) \\
\hline Mean & 56 & 368 & 57 & 407 & 58 & 406 \\
CV & $3.03 \%$ & $1.36 \%$ & $0.51 \%$ & $0.26 \%$ & $1.12 \%$ & $0.56 \%$ \\
Error & $19.2 \%$ & $33.9 \%$ & $17.2 \%$ & $48.1 \%$ & $15.8 \%$ & $47.8 \%$ \\
\hline
\end{tabular}

Group one samples demonstrated a large CV and smaller absolute values than those of group two or three. This was anticipated as the inaccurate and irregular nature 
of cutting with a band saw induces a rough surface finish promoting varied and premature failure. The results of group two and three testing are very similar with the most discernible difference being the required toe-compensation, due to differences in take-up of slack and alignment between testing sessions. Refer to Figure D.1 in Appendix D for an equivalent, non-toe-compensated plot. Minute variation in strengths and moduli are anticipated to be a result of small disparities in sample geometry, sample alignment with the fixture or material variability. Nevertheless, all three groups showed moduli and strength values with a large percentage error between experimental and theoretical values. This observed lack of accuracy may be due to undocumented material inhomogeneity (e.g. surface treatment) resulting in divergence between tensile and flexural properties or an overcompensation employed by the manufacture to ensure mechanical properties are greater than or equal to nominal values, given an appropriate tolerance. While applied strain rates were $21 \%$ to $27 \%$ slower than those specified by ASTM standard, such a difference would demonstrate an inconsequential effect on strength and no effect on modulus. Furthermore, a decrease in strain rate would theoretically decrease yield strength [94]. This observed precision but lack of accuracy is acceptable for testing intended to characterize the relativistic effects of various environmental exposures on the flexural properties of the material in question however, should not be used to specify design parameters. Hence, testing with the flexural fixture shall be for the characterization of relative effects on flexural properties unless subsequent evaluation indicates the perceived error is a function of the material or sample geometry rather than inherent to the test fixture. 


\subsection{Coupon Manufacturing}

Test specimens were manufactured from an eight-harness satin weave, AX-5180 curing epoxy-carbon prepreg produced by Axiom Materials, Inc. AX-5180 was selected for its recognized industrial adoption as a structural laminate for aircraft interior applications, due to its low flammability characteristics. While the exact composition of AX-5180 is proprietary, Table 2.2 describes its approximate composition.

Table 2.2: Chemical composition of AX-5180 prepreg, adapted from [1].

\begin{tabular}{ccc}
\hline Ingredient & CAS NO. & \% WT \\
\hline Poly(Bisphenol A-co-epichlorohydrin) & $25036-25-3$ & $<20 \%$ \\
$($ Epoxy Resin) & & \\
Poly[(phenyl glycidyl ether)-co-formaldehyde $]$ & $28064-14-4$ & $<20 \%$ \\
(Epoxy Resin) & & \\
Epoxy Resin Copolymer & Proprietary & $<20 \%$ \\
Fumed Silica & $67762-90-7$ & $<3 \%$ \\
Cyanoguanidine & $461-58-5$ & $<3 \%$ \\
Epoxy Curing Agent & Proprietary & $<3 \%$ \\
Flame Retardant & Proprietary & $<20 \%$ \\
Carbon & $7440-44-0$ & $<60 \%$ \\
\hline
\end{tabular}

Prepreg consisted of $54 \%$ fibre content by volume ( $60 \%$ by weight) as indicated through communication with the industrial partner. Flexural samples were acquired from a monolithic laminate manufactured with a $\left[0_{8 \mathrm{~F}}\right]$ stacking sequence (eight fabric plies with warp fibres in the $0^{\circ}$ direction) and was cured using a two-temperature stage, autoclave process depicted in Figure 2.3. This cure cycle, implemented in a early design stage, yielded laminate with a void content of $2.9 \%$, as determined by microtomographic analysis. 


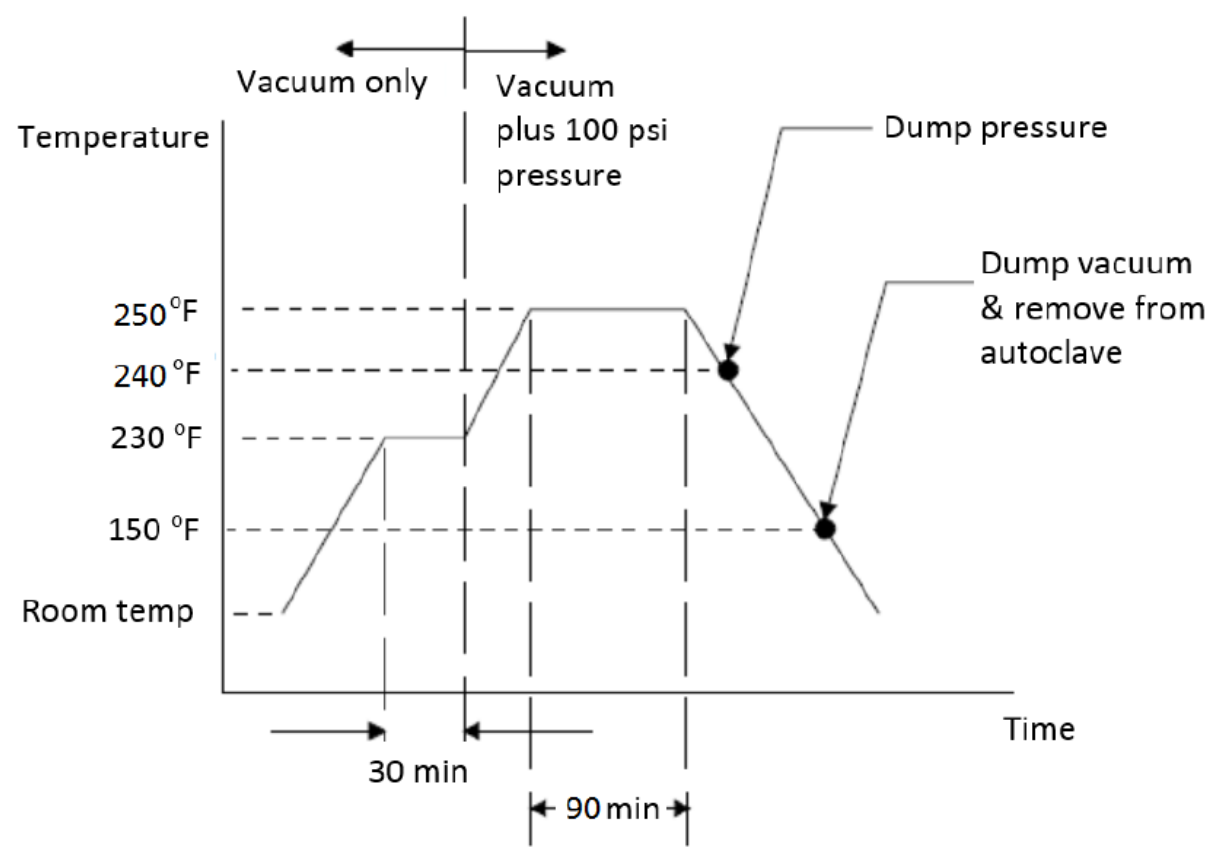

Figure 2.3: Cure cycle used for flexural coupon parent laminate.

SBS samples were extracted from a monolithic laminate manufactured with a $\left[0_{2} / \pm 45 / 0 / 45\right]_{\mathrm{S}}$ stacking sequence (two $0^{\circ}$ plies; two $45^{\circ}, 90^{\circ}$ to one another; one $0^{\circ}$ ply; and one $45^{\circ}$, a symmetric, mirror image stacking sequence reflected about the center plane) with a single [0]-orientation, non-structural, conductive mesh and fibreglass on the tool and bag surfaces, respectively. A laminate's tool surface corresponds to the external surface of the ply which was in contact with the tool (or mold) surface during curing and its bag surface corresponds to the ply surface in contact with vacuum bag during curing. The laminate plate fabricated for SBS samples was cured using a later-stage design cycle, and thus yielded a lower void content- $0.24 \%$ as determined through optical microscopy. The SBS parent laminate was cured using an improved curing cycle depicted in Figure 2.4, with vacuum, pressure and temperature plotted relative to time. Layup and curing processes were performed by third party manufacture, Stelia Aerospace.

Test specimens were cut using a water-cooled diamond blade on a UKAM Smart 


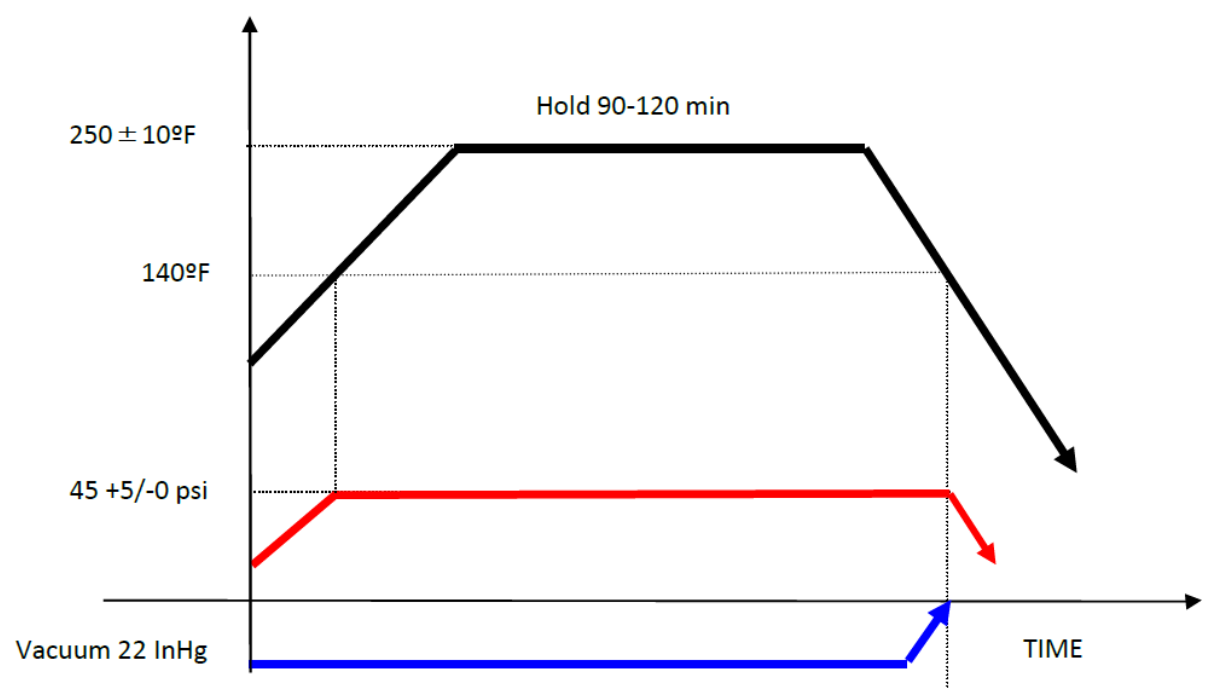

Figure 2.4: Cure cycle used for SBS coupon parent laminate.

Cut 6001 GP Sectioning Saw. Flexural samples were cut to a 32:1, span-to-thickness ratio with a 0.5-inch width, in accordance with ASTM D7264 standard [86]. Allowing an additional $20 \%$ in length for overhang at the sample supports, samples measured on average $4.786 \times 0.496 \times 0.126$ inches with standard deviations of $0.024,0.014$ and 0.001 inches, respectively. SBS samples were cut to a 6:2:1, span-to-width-tothickness ratio, in accordance with ASTM D2344 standard [88]. On average, SBS samples measured $0.989 \times 0.325 \times 0.164$ inches with standard deviations of 0.022 , 0.016 and 0.001 inches, respectively. After cutting, flexural and SBS samples were assigned an alphanumeric label identifying them based on their location in the parent laminate and marked accordingly with an opaque, white marker and pencil crayon, respectively. Figure 2.5 presents both flexural and SBS samples, contrasting their size and structure while orthographic projections presented in Appendix C display associated dimensional tolerances. 


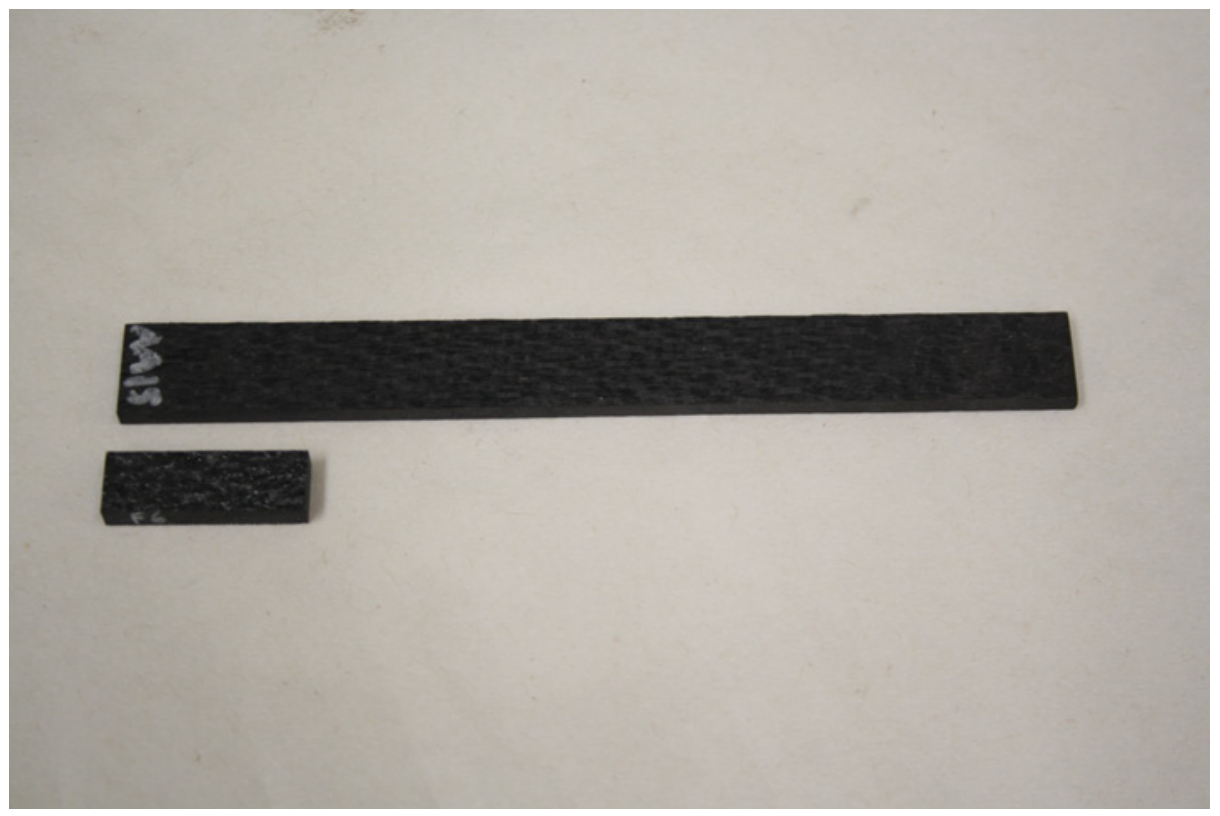

Figure 2.5: Image of representative flexural (AA15) and SBS sample (F6).

\subsection{Sample Conditioning}

Sample conditioning took place at $85^{\circ} \mathrm{C}$ in a Quincy Lab Inc., 30AFE-LT, low temperature, forced air laboratory oven. The laboratory oven had an internal volume of 1.78 cubic feet $(18 \times 12.2 \times 12$ inches $)$ and a temperature range from ambient to $107^{\circ} \mathrm{C}$. Flexural samples were conditioned through single-sided exposure using a custom-designed fixture, while SBS samples were condition through immersion with samples individually contained, in an array of test tubes. The following describes the design, validation and finalized setup of the single-sided conditioning fixture followed by a detailed description of the SBS conditioning setup.

\subsubsection{Single-Sided Conditioning Fixture}

In order to determine the rate of one-dimensional moisture absorption in a composite, fluid must be restricted such that it may only permeate the material of interest 
through a single surface. In addition, conditioning must be performed on individual samples such to avoid confounding variability related to sample preparation following conditioning and extended periods during which the sample is not exposed following conditioning, but prior to testing. While methods have been developed for the purpose of exposing select surfaces to a fluid $[22,58,95]$, they are not well suited for longterm exposures, elevated temperatures, small sample dimensions, volatile fluids, or a combination of these factors. Hence, such a situation necessitated a conditioning fixture designed to meet the identified shortcomings in existing methods.

\section{Design}

To expose only a single surface to the fluid of interest, a fixture was designed which confines the fluid to a volume on top of each sample. Figure 2.6 illustrates a crosssection of the proposed design. This design consists of four distinct layers: the topplate, which contains a sequence of wells designed to hold liquid during conditioning; gasket, which forms a moisture tight seal between the composite samples and the topplate; composite samples; and bottom-plate, which seats each sample relative to the gasket and top plate. To both ensure alignment and produce an even pressure over each sample's exposed surface, a grid of fasteners (i.e. screws, nuts and washers) were positioned around wells. To ensure an even pressure, a torquing sequence was applied in a raster pattern. Figure 2.7 illustrates a conceptual schematic of an early-stage conditioning fixture (array), designed to house all samples from a single exposure duration. 


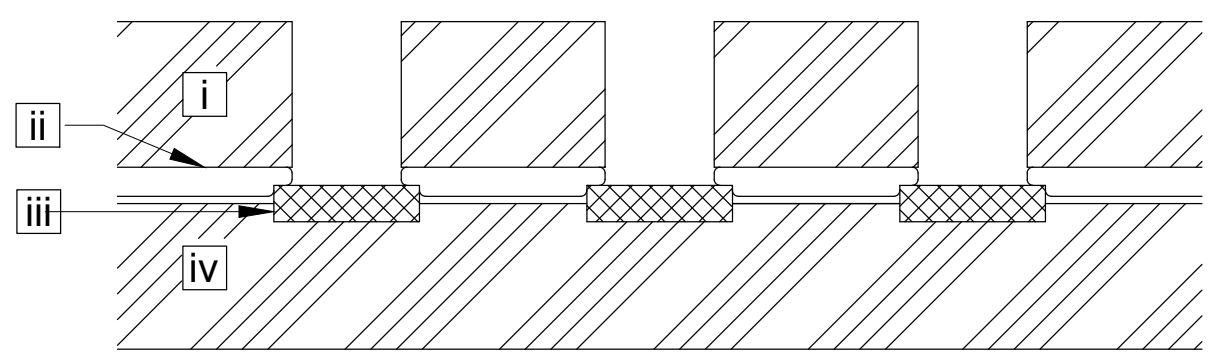

Figure 2.6: Cross-section of proposed conditioning fixture illustrating i) top-plate, ii) gasket, iii) sample and iv) bottom-plate.

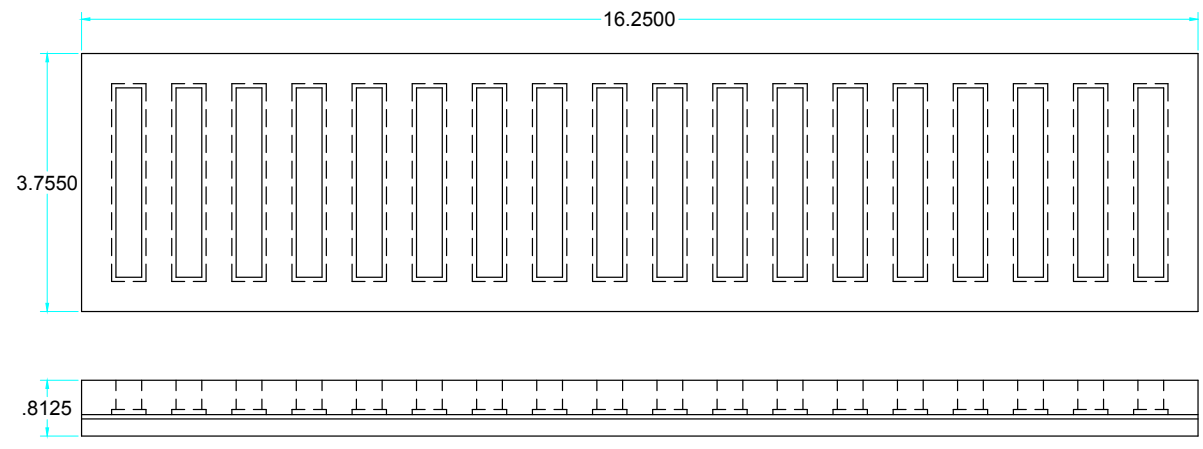

Figure 2.7: Preliminary design of 18-well conditioning array.

\section{Material Selection}

In designing a fixture for conditioning, it was important to select materials which would be able to withstand elevated temperatures and chemical exposure without physical or chemical degradation, as either could prevent the fixture from maintaining single-surface exposure or contributing confounding compounds to the fluids, preventing the formation of credible conclusions. Components of the fixture had to possess properties which allowed them to fulfill their function. The gasket material had to compressible and deformable such that it retained a good seal both at the sample surface and top plate boundaries. The top and bottom plates had to be machinable and relatively rigid such that they retained their shape and effectively transfer force from the torque of the fasteners to the gasket. Additionally, all components had to be readily available, economical and nontoxic. 
To simplify the search, reduce final cost and restrict overall mass, material selection was limited to engineering polymers. To identify acceptable materials a comparison of chemical resistances to the selected fluids was performed using supplier-published, qualitative, chemical compatibility charts [96-99]. Following this, materials which were sufficiently chemically inert were further compared by their thermal and mechanical properties. Based on these attributes, potentially viable materials were selected, priced and sourced.

Polytetrafluoroethylene (PTFE), also known by its brand name Teflon, was selected for the bottom and top-plates due to its recognized, near comprehensive chemical resistance, acceptable thermal properties $\left(T_{m}\right.$ and $T_{g}$ greater than $\left.100^{\circ} \mathrm{C}\right)$ and modest flexural modulus. Due to contradictory opinions on chemical resistance, both high-temperature nitrile rubber (also known as Buna-N) and silicone (Poly(dimethylsiloxane)) rubber were selected to be tested as potential gasket materials. Both materials offered sufficiently low Shore-D hardness values and acceptable thermal properties, however, both had sources which claimed they were chemically incompatible with one of the fluids being investigated; silicone potentially susceptible to turbine oil and nitrile potentially susceptible to synthetic hydraulic fluid. In order to evaluate their susceptibility, small sample sheets of each material were procured, sheared into 1/4 inch-diameter cylinders and immersed in their respective fluids. Then, each was placed in the laboratory oven at $85^{\circ} \mathrm{C}$ and left to age for nine days. Samples were then qualitatively assessed by being compared to a non-exposed control, inspected for visual and tactile changes. From this, neither material was found to discernibly degraded thus, silicone was selected due to its lower cost.

316 stainless steel fastener components were selected for their recognized chemical resistance, temperature stability, strength, and commercial availability. 


\section{Test Array Trial}

In order to validate that the proposed design would function as anticipate, a smallscale test array was fabricated. Top and bottom-plates were machined on a manual milling machine and gasket material was cut using a hobby knife. Once fabricated, the test array was assembled and assessed for its ability to hold and retain water, both at room temperature and $85^{\circ} \mathrm{C}$. To do this, sample wells were filled with coloured water to reveal cell cross-contamination, and left to sit both at $85^{\circ} \mathrm{C}$ and ambient room temperatures.

From this test, a number of conclusions were drawn. First, fasteners were placed too sparsely. As a result of PTFE's flexibility, even at room temperature, array leaking and cell cross-contamination was evident. Second, with a modest torque applied to fasteners the bottom-plate, which was 1/8-inch-thick, would plastically deform and retain its deformation when loading was removed. This deformation was not evident in the $1 / 2$-inch-thick top-plate. Lastly, due its relatively high volatility, at $85^{\circ} \mathrm{C}$, water would evaporate from the cells faster than feasible for an inspection frequency. From these inferences, changes were made to the array design for subsequent iterations. Figure 2.8 exhibits the test array with clamps affixed to test alternative fastener positioning.

\section{Array Fabrication}

Figure 2.9 shows an assembly of the final, full-scale array. Six full-scale arrays were fabricated-PTFE components were machined using a CNC milling machine and gasket material was cut with a hobby knife. Outer dimensions were selected to maximize the number of samples per array while allowing for airflow in the laboratory convection oven. Improving from the test array, the bottom-plate was increased to be 


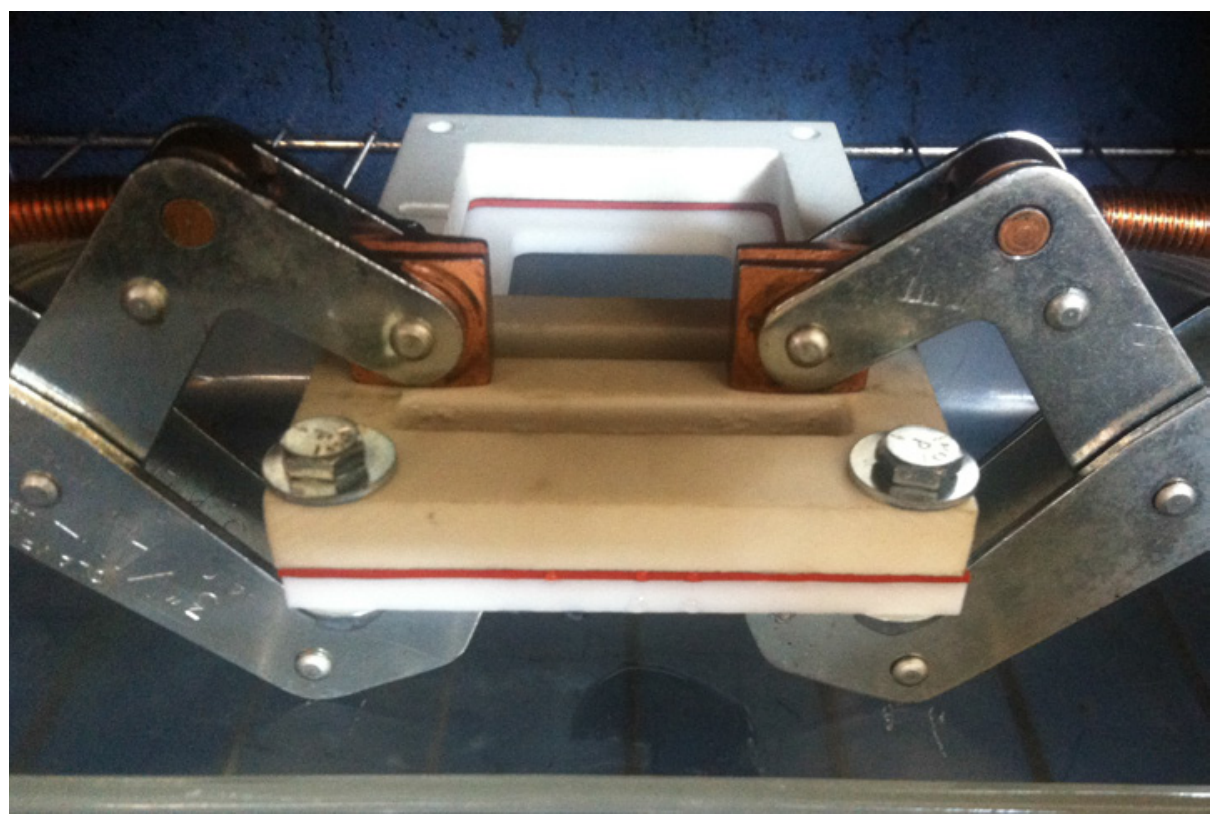

Figure 2.8: Small-scale test array with clamps placed to prevent cell crosscontamination.

1/2-inch-thick, reducing pressure induced deformation; gasket material was increased from $1 / 8$ to $1 / 4$-inch thick and procured at a lower durometer value to ensure compliance; and fastener frequency and positioning was improved to a establish a more even pressure distribution.

Figure 2.10 shows the assembled conditioning array structure, exhibiting top-plate, gasket and bottom-plate; samples wells; and fastener layout. Orthographic projection drawings of the array's top and bottom PTFE plates have been presented in Appendix C.

\section{Fluid Retention}

Similar to the small-scale trial, the full-scale conditioning arrays were evaluated on their capability to retain fluids in their sample wells without leaking or well crosscontamination, while also limiting well evaporation. As the lowest viscosity and 


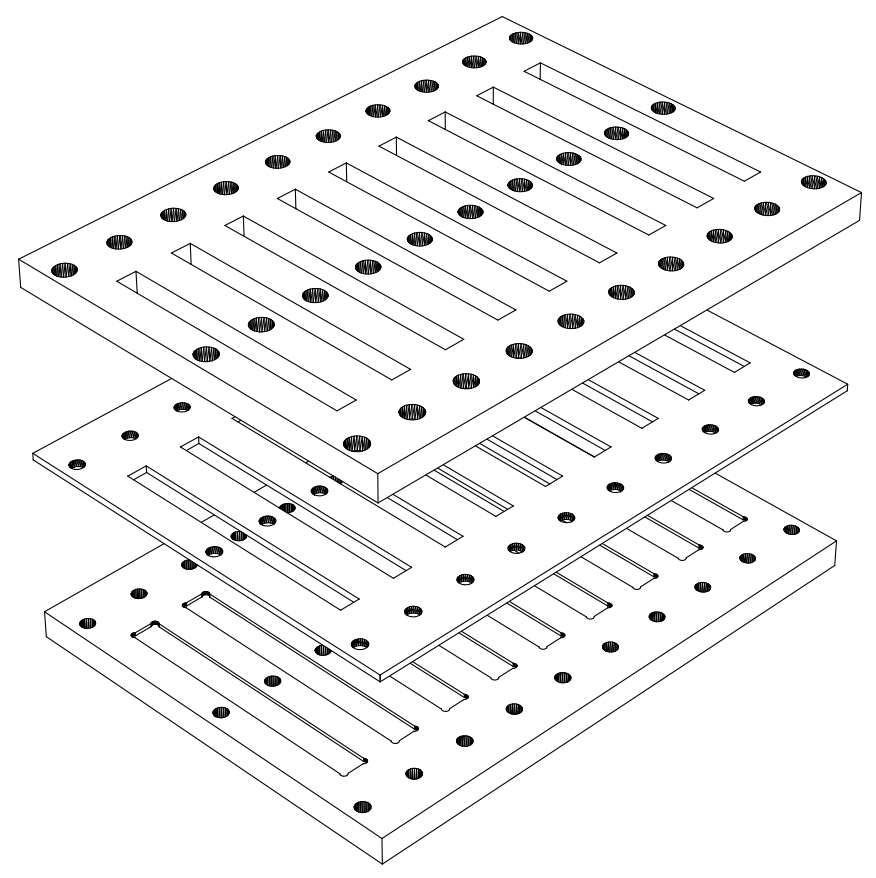

Figure 2.9: Full-scale array assembly.

highest volatility liquid, water was selected for validation trials. First, a fully assembled array, containing composite samples, had its wells filled with tap water at room temperature. Systematically, in $2.5 \mathrm{in} \cdot \mathrm{lb}$ increments, the array was tested with torquing patterns of increasing force, to determine the ideal torque; one ensuring fluid retention while limiting deformation. It was conservatively decided that the array would be torqued to its final force through a single-stage, raster pattern repeated three times. From this, it was determined that a $10 \mathrm{in} \cdot \mathrm{lb}$ torque was sufficient to retain liquid in all wells at room temperature. The arrays were then torqued to $10 \mathrm{in} \cdot \mathrm{lb}$ and placed in the laboratory oven, at $85^{\circ} \mathrm{C}$, for 24 hours to allow thermal equilibrium to be obtained. Once met, sample wells were again filled with tap water and cover with a vinyl adhesive tape - limiting evaporation — and left at temperature. Daily, the arrays were inspected to ensure well liquid levels were sufficient to keep the composite surface immersed and to verify that the induced change in viscosity 
Figure 2.10: Assembled array structure.

would not allow the water to drain from the wells (as indicated by the presence of dye trailing from the gasket-plate contact surface). From this, it was demonstrated that, over a period of two weeks, the full-scale array was capable of retaining liquid without leaks or well cross-contamination. Furthermore, while the applied tape aided in limiting evaporation, it did not prevent it, requiring additional liquid be added approximately every three days.

A final retention test validated the results of the previous, water-only evaluation, while testing all conditioning fluids, at temperature, over a period of a week. Additionally, the vinyl adhesive tape was replaced with a transparent polyimide, silicone adhesive tape to both, allow inspection of the contained fluids and to limit the risk of adhesive degradation confounding results. Polyimide tape was selected due to its availability and low-cost, however, fluid visibility and retention in the sample wells may have been more effectively achieved using optical flats. While the test presented similar results, it demonstrated that the low volatility of the hydraulic fluid and turbine oil 
resulted in negligible evaporation over the test period, while ethylene glycol showed an intermediary level of evaporation. Thus, during full scale-testing, sample wells containing these fluids required less frequent filling. Figure 2.11 demonstrates the full-scale array following testing with polyimide tape applied.

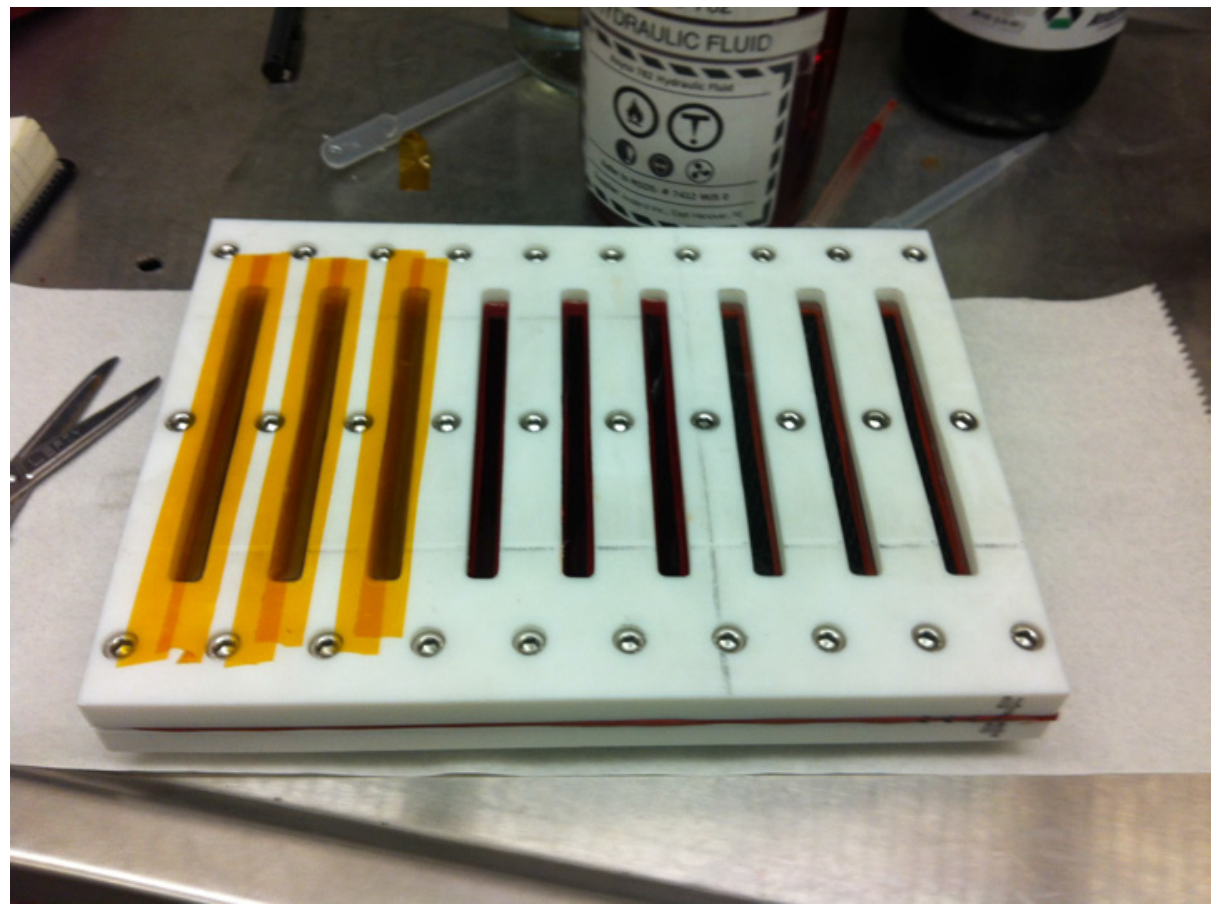

Figure 2.11: Array during fluid retention testing with polyimide tape applied over select wells.

\section{Temperature Distribution}

In order to ensure obtained results were not confounded by variation in temperature between arrays, a sequence of tests were carried out to map the heat distribution within the laboratory convection oven when sample arrays were contained. In accordance with ASTM D5229 [95], which describes standard methods by which to determine moisture absorption properties of polymer matrix composites, conditioning should maintain the required temperature $\pm 3^{\circ} \mathrm{C}$, hence, the goal of these tests were to find an array configuration in the oven which could meet this criteria once 
thermal equilibrium was obtained. By placing each of the six sample arrays in the oven on separate shelves, adhering a J-type thermocouple to each and measuring their equilibrium temperature, the induced distribution and average temperature was determined.

Making micro-adjustments to oven set-point temperature, oven contents, and the relative $\mathrm{x}-, \mathrm{y}$ - and $\mathrm{z}$-coordinates of the arrays, a configuration was identified which minimized temperature distribution and maximized the number of samples which could be simultaneous conditioned, while achieving an average temperature of $85^{\circ} \mathrm{C}$. No configuration involving all six sample array could feasibly maintain $85 \pm 3^{\circ} \mathrm{C}$ as the temperature discrepancy between the bottom array and the top array was too great. This was due to poor air circulation, a result of the large surface area arrays. The finalized configuration used four sample arrays placed centrally in the oven as illustrated in Figure 2.12. A sheet of nitrile rubber was placed between the bottom array and the heating coils, promoting air flow around the out sides of the arrays and stifling direct transmission of heat to the bottom array. To further minimize array temperature differences, it was decided that each array would change oven position (i.e. positions 1, 2, 3 and 4 in Figure 2.12) every three days, in conjunction with sample mass measurements. In this manner, samples conditioned for longer durations had a near equivalent exposure time to the average temperature of each array position, allowing each to be exposed to the same average temperature while sacrificing temperature variance.

\subsubsection{SBS Conditioning}

Unlike flexural samples, SBS samples were conditioned though complete immersion, contained in $18 \times 150 \mathrm{~mm}$ test tubes. Each sample was placed in an upright position 


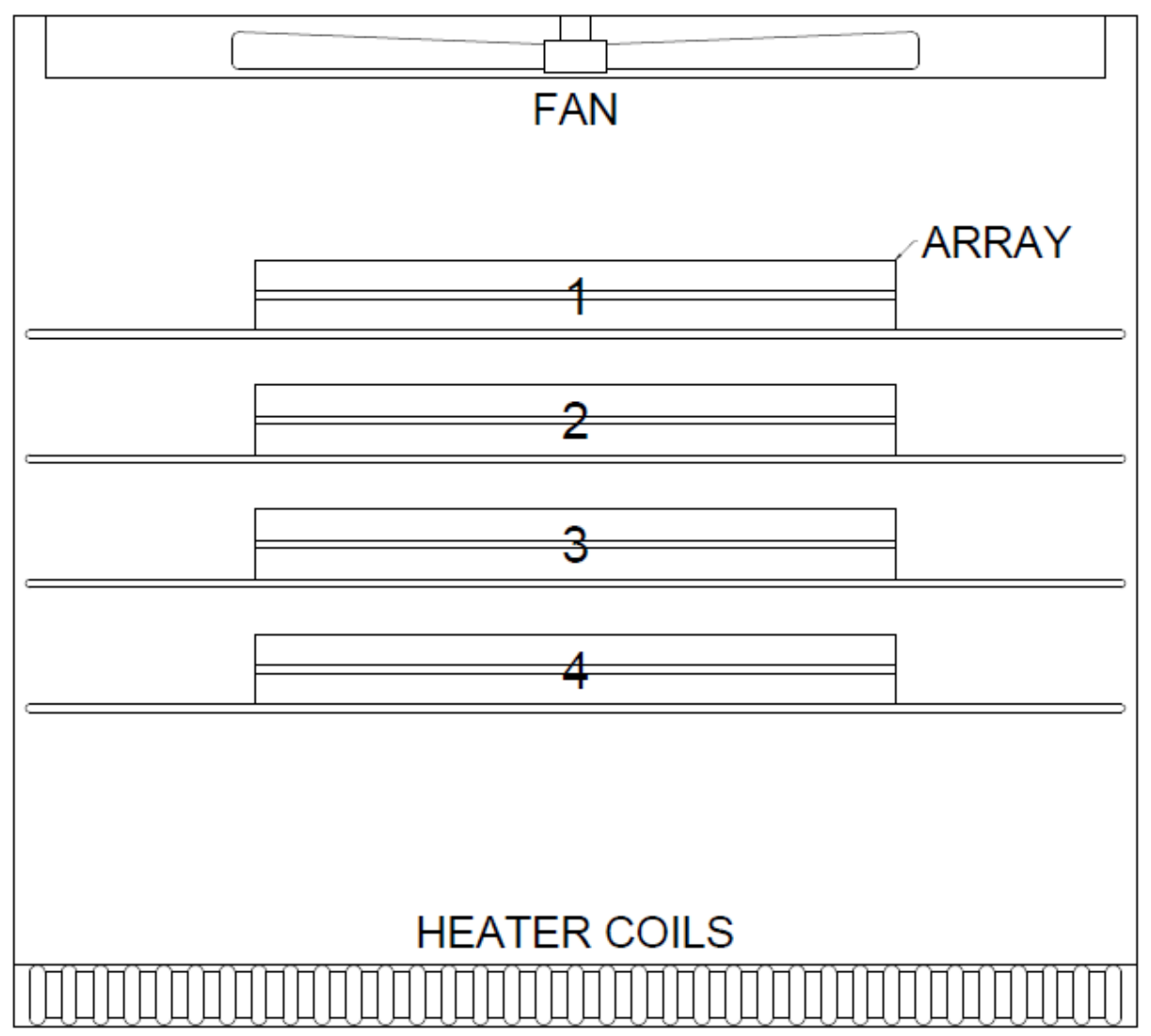

Figure 2.12: Schematic of finalized oven configuration.

in a test tube and immersed in liquid. Selected test tubes possessed hemispherical bottoms, limiting the contact surface between SBS samples and the test tube. Test tubes were filled one-third-full with their respective liquids and capped with neoprene rubber stoppers to reduce evaporation of volatile liquids. Placed centrally in the laboratory oven, the test tube array demonstrated the capability to maintain a temperature distribution of $85 \pm 2^{\circ} \mathrm{C}$. Figure 2.13 illustrates the SBS-test tube, conditioning setup and Figure B.1 in the Appendix B depicts the relative positioning of liquids in the test tube rack. 


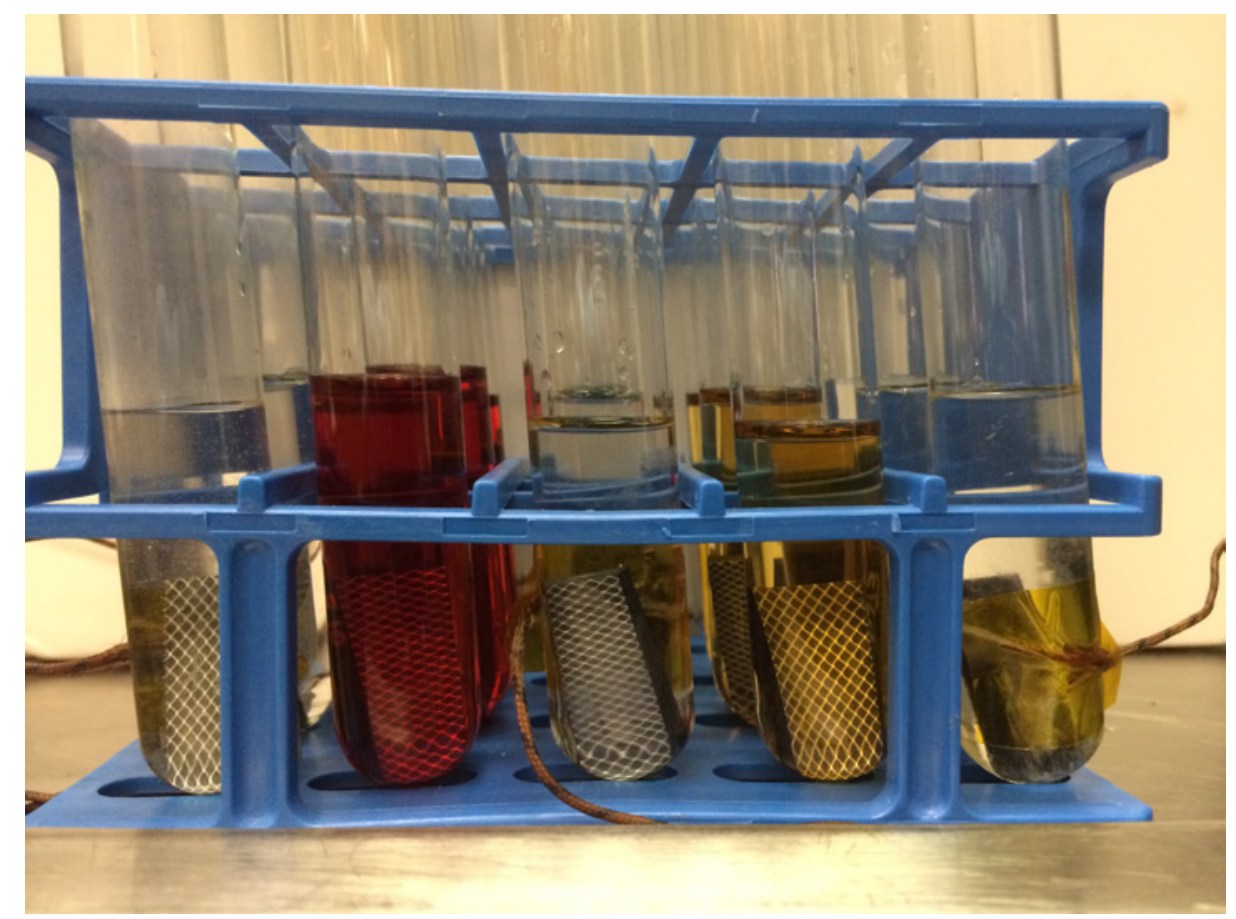

Figure 2.13: SBS samples immersed in fluids, positioned in test tube rack.

\subsection{Moisture Uptake}

As discussed in the literature review, Fickian diffusion is the predominant model used to describe the uptake of moisture into a composite over time, as manifested through sample mass gain. Fickian diffusion can be described by two laws; Fick's first law, which describes diffusant flux as a function of diffusivity and concentration gradient under a steady-state condition and Fick's second law, which relates the change in concentration per unit time to diffusivity and the second derivative of the concentration gradient. Fick's laws may be expressed for multi-dimensional diffusion however, for both its relevance to the work described and its simplicity, they will be described here in one dimension, normal to the laminate planes. Fick's first law may be described by the expression 


$$
J=-D \frac{d C}{d x}
$$

where $J$ is the diffusion flux expressed in terms of mols of diffusant per area per time, $D$ is the diffusion coefficient (mass diffusivity) expressed in terms of area per time, $C$ is the concentration of the diffusant in mols per volume and $x$ is position. $D$ is assumed to be constant for a specified diffusant-medium combination, temperature and pressure. Generally, it is unrealistic to view a system under a constant concentration gradient and one must evaluate the change in concentration with time. To do this, Fick's second law must be considered. Fick's second law is described by the partial differential equation

$$
\frac{\partial C}{\partial t}=D \frac{\partial^{2} C}{\partial x^{2}}
$$

where $t$ represents the advancement in time from initiation $(t=0)$. Given a linear case for a finite system, here a laminate plate with diffusant in contact with both external surfaces with normal vectors perpendicular to the laminate planes and not the laminate's edges (exposed surfaces with normal vectors parallel to laminate planes), represented by the boundary conditions

$$
\begin{gathered}
C=C_{i} \quad \text { for } \quad 0<x<h, \quad t \leq 0, \\
C=C_{a} \quad \text { for } \quad x=0 ; x=h, \quad t>0 .
\end{gathered}
$$

Where $C_{i}$ is the initial concentration, $C_{a}$ is the concentration at some time $a, t>0$ and $h$ is the thickness of the laminate plate, Jost has proposed a numerical solution to Fick's second law [100], expressed as 


$$
\frac{C-C_{i}}{C_{\infty}-C_{i}}=1-\frac{4}{\pi} \sum_{i=0}^{\infty} \frac{1}{2 i+1} \sin \frac{(2 i+1) \pi x}{h} \exp \left[-\frac{(2 i+1)^{2} \pi^{2} D t}{h^{2}}\right]
$$

Here, $C_{\infty}$ is the concentration of diffusant at saturation. To express this relationship in terms of change in absorbed mass, $m$, rather than concentration, Equation 2.9 may be integrated over the plate's thickness

$$
m=\int_{0}^{h} C d x
$$

to obtain

$$
G \equiv \frac{m-m_{i}}{m_{\infty}-m_{i}}=1-\frac{8}{\pi^{2}} \sum_{i=1}^{\infty} \frac{\exp \left[-(2 i+1)^{2} \pi^{2}\left(\frac{D t}{h^{2}}\right)\right]}{(2 i+1)^{2}}
$$

where $G$ is defined to be a dimensionless-, time, material, pressure and temperature dependent, moisture absorption function. As demonstrated by Shen and Springer [101], this equation may be approximated as

$$
G=1-\exp \left[-7.3\left(\frac{D t}{4 h^{2}}\right)^{0.75}\right]
$$

for single-sided moisture exposure. Rearranging Equation 2.11 as

$$
M=G\left(M_{\infty}-M_{i}\right)+M_{i}
$$

where $M$ is moisture content (percentage change in mass), allows for expression of change in absorbed mass as a function of $G$, initial moisture content, $M_{i}$, and moisture 
content at saturation $M_{\infty}$. Hence, in order to model moisture diffusion in a composite based on presumed Fickian diffusion, known values of laminate thickness, initial mass, mass at moisture saturation and the diffusion coefficient are required. While the laminate thickness is a design parameter and initial moisture content can be controlled through sample drying, the diffusion coefficient and moisture content at saturation must be determined empirically. Moisture content at saturation may be determined by saturating a sample and measuring change in mass; saturation is characterized by the point at which the passing of time does not result in a change in sample mass. The diffusion coefficient may be approximated knowing the slope of the initial linear region of a Fickian absorption curve which may be identified by plotting the change in mass as a function of the square root of time. As described by Chen and Springer [101], the diffusion coefficient for single-sided diffusion may be approximated as

$$
D=\pi\left(\frac{2 h}{4 M_{\infty}}\right)^{2}\left(\frac{\Delta M}{\Delta \sqrt{t}}\right)^{2}
$$

The standard test method for evaluating the absorption characteristics, establishing moisture equilibrium, $M_{\infty}$, and Fickian moisture diffusion properties has been described in ASTM D5229 [95]. While describing a number of case specific methods, this standard is based upon the determination of moisture content from changes in sample mass, as represented by

$$
\Delta m=\frac{m-m_{0}}{m_{0}} \times 100
$$

where $m_{0}$ is the baseline sample mass. This method, while being the most common for evaluation of moisture absorption, faces the caveat that it cannot provide diffusion 
specific inferences if the material under investigation does not exhibit Fickian diffusion. In order for diffusion to be classified as Fickian it must satisfy three conditions, as offered by Shirrell [102]:

1. absorption and desorption are linear up to $\geq 60 \%$ of the saturation moisture content when plotting moisture absorption vs. $\sqrt{\text { time }}$

2. beyond the linear portion, absorption and desorption curves are concaved to the $\mathrm{x}$-axis until the saturation moisture content is reached

3. For fixed values of initial and final concentrations, sorption curves for specimens of different thicknesses are superimposable when plotting moisture sorption vs $\sqrt{\text { time } / h}$

Whether a material system will adhere to these conditions is unknown prior to testing. Thus, while following this standard allows for determination of a moisture absorption as a function of time, it cannot assure the collected data can be modeled. Regardless, ASTM D5229 served as a guide for evaluation of moisture absorption for this work.

As discussed in Section 1.3.2, it is important that the results of accelerated ageing experiments can be correlated to in-service conditions. Hence, it is desirable to be able to extrapolate obtained data to other exposure temperatures. Equation 2.14 approximates the diffusion coefficient given a constant temperature. Thus, to estimate diffusion rates for different temperatures, the temperature dependent diffusion coefficient must be approximated. The Arrhenius equation, represented as

$$
D(T)=D_{0} e^{\left(-E_{d} /(R T)\right)}
$$

allows for prediction of the diffusion coefficient at varied temperatures, where $D_{0}$ is 
the permeability index $\left[\mathrm{m}^{s} / \mathrm{s}\right], E_{d}$ is the activation energy for diffusion $[\mathrm{J} / \mathrm{mol}], R$ is the universal gas constant $[J /(\mathrm{mol} \cdot K)]$ and $T$ is temperature $[K]$. As the work entailed did not allow for determination of permeability index or the activation energy of diffusion, these values were approximated from literature, allowing for a prediction of a generalized diffusion scenario and the prediction of the investigated composite's diffusion characteristics at different temperatures, assuming proportionality.

\subsubsection{Metrology}

Moisture absorption was assessed through determination of sample mass using a Sartorius $A G$, ENTRIS64-1S ( $\pm 0.0001 \mathrm{~g})$ analytical balance, at strategic intervals. At the time of mass evaluation, length, width and thickness measurements were evaluated using a digital caliper $( \pm 0.001 \mathrm{in})$. This was done in effort to estimate the linear coefficients of moisture expansion, $C M E$,

$$
C M E=\frac{\Delta L / L_{0}}{\Delta m / m_{0}}
$$

where $\Delta L$ is change in length, $L_{0}$ is the initial length and $\Delta m$ is the change in mass and $m_{0}$ is the initial mass. While caliper resolution restricted the accuracy of sample dimensions, relative changes noted between chemical groups allowed for inferences regarding the mechanism volume change and its effect on interpreted mechanical properties. 


\subsection{Supplementary Evaluation}

Supplementary analysis was carried out to gain additional information pertaining to the degradation of the FRPMC under investigation. This includes qualitative observations to support changes in flexural data, mass uptake and metrology; insight into potential degradation mechanisms; and evaluation of techniques for inspecting exposed material. Optical microscopy was performed to observe macroscopic damage to the flexural samples' surfaces. Hardness testing was carried out to evaluate its usefulness as a minimally invasive inspection technique for the detection of moisture uptake. Micro-computed tomography was employed to evaluate the internal structure of the flexural samples, comparing pre-exposure and post-exposure. Finally, thermal analysis, including DSC and TGA, was utilized to investigate changes in glass transition temperature, degradation temperature and to compare volatile mass fraction to observed mass uptake to ascertain the physisorption or chemisorption tendencies of tested fluids within the FRPMC. 


\section{Chapter 3}

\section{Results}

This chapter presents the results of both flexural and SBS test campaigns, and ac-

companying discussion. Arranged by campaign, the results of single-sided flexural experiments are presented first, systematically addressing moisture absorption, sample metrology, flexural properties, surface microscopy and x-ray micro-computed tomography. Subsequently, the results of immersion SBS experiments are presented, examining moisture absorption, short beam strength, hardness measurements and thermal analysis. Sections of the second campaign addressing the same subjected matter as the first will compare and contrast SBS results with those obtained from flexural testing. All plots and discussion presenting a percentage change of a parameter compare conditioned samples with respect to samples prior to conditioning or an equavalient, unexposed sample group, unless otherwise stated. 


\subsection{Campaign One: Single-Sided Flexural}

\subsubsection{Moisture Absorption}

Prior to conditioning, samples were dried to establish an essentially moisture-free baseline sample mass, $m_{B}$, in accordance with ASTM D5229, Procedure $D$ [95]. Table 3.1 outlines the specifications of Procedure $D$ and any required deviations. Changes made were driven by equipment availability and available time for experiments.

Table 3.1: Specified and utilized test parameters for ASTM D5229, Procedure D; deviations from the specified standard highlighted in bold.

\begin{tabular}{|c|c|c|}
\hline Test Parameter & Specification & As Tested \\
\hline Number of replicates & Three & Three \\
\hline Mass precision & $\pm 0.0001 \mathrm{~g}$ & $\pm 0.0001 \mathrm{~g}$ \\
\hline Temperature & Study specific & $50{ }^{\circ} \mathrm{C}$ \\
\hline Oven exterior environment & Standard laboratory environment & Laboratory fumehood \\
\hline Weighing reference periods & 7 Days & 3 Days \\
\hline Cooling, pre-weighing & Contain in a desiccator & $\begin{array}{l}\text { Contained in sealable plastic } \\
\text { bags }\end{array}$ \\
\hline Moisture equlibrium & $\begin{array}{l}<0.02 \% \text { change over two } \\
\text { reference periods }\end{array}$ & $\begin{array}{l}<0.02 \% \text { change over one } \\
\text { reference period }\end{array}$ \\
\hline Out-of-oven time & $<30$ minutes per weighing & $<30$ minutes per weighing \\
\hline Post-dry storage & Contain in a desiccator & $\begin{array}{l}\text { Contained in two sealable plastic } \\
\text { bags, the outer containing desiccant }\end{array}$ \\
\hline
\end{tabular}

Once dried, samples were conditioned in accordance with ASTM D5229, Procedure $B$ [95]. It is important to note that Procedure $A$ describes the determination of Fickian moisture diffusion material properties while Procedure $B$ outlines non-ambient moisture conditioning of material specimens prior to other types of testing. As Procedure $A$ requires specific coupon dimensions that do not match those required by ASTM D7264 [86], as was necessary for subsequent flexural testing, Procedure $B$ was followed and adapted to allow for determination of Fickian moisture diffusion 
material properties. Procedure $B$ outlines a number of conditioning-specific variants, thus, methods were adapted from procedures $B W F F$ and $B F F F$ which describes conditioning samples through $\mathbf{W}$ ater and Fluid immersions for a Fixed time period with a Fixed weighing interval. Table 3.2 outlines the specifications of Procedure $B$.

Table 3.2: Specified and utilized test parameters for ASTM D5229, Procedure $B$; deviations from the specified standard highlighted in bold.

\begin{tabular}{|l|l|l|}
\hline \multicolumn{1}{|c|}{ Test Parameter } & \multicolumn{1}{c|}{ Specification } & \multicolumn{1}{c|}{ As Tested } \\
\hline Number of replicates & Three & Three \\
\hline Sample Mass & $\geq 5 \mathrm{~g}$ & $\pm 5 \mathrm{~g}$ \\
\hline Mass precision & $\pm 0.0001 \mathrm{~g}$ & $85^{\circ} \mathrm{C}$ \\
\hline Temperature & Study specific & Laboratory fumehood \\
\hline Oven exterior environment & Standard laboratory environment & Procedure D \\
\hline Preconditioning & Procedure D & Single-sided \\
\hline Conditioning & Immersion & $\begin{array}{l}\text { Contained in sealable } \\
\text { plastic bags }\end{array}$ \\
\hline Cooling, pre-weighing & Contain in a specimen bag & $\begin{array}{l}\text { Wipe with lint-free towel, } \\
\text { SOP detailed in B.1 }\end{array}$ \\
\hline Surface moisture removal & Wipe with lint-free towel & \\
\hline
\end{tabular}

Moisture absorption curves plotted in Figure 3.1 summarize percentage change in sample mass as a function of conditioning duration, expressed in $\sqrt{\text { hours }}$, for the five chemicals sample and control samples investigated. For clarity, reference to control samples hereafter refers to samples which were conditioned at elevated temperatures without fluid exposure for equivalent durations as their fluid exposed counterparts, whereas baseline samples refers to samples which were dried to moisture equilibrium but were not conditioned at elevated temperature for prolonged durations nor exposed to fluids. Each data point represents the average percentage change in mass of three samples for a given duration, with the exception of the one-month control group which is based on the average of two samples due to sample well cross-contamination. 


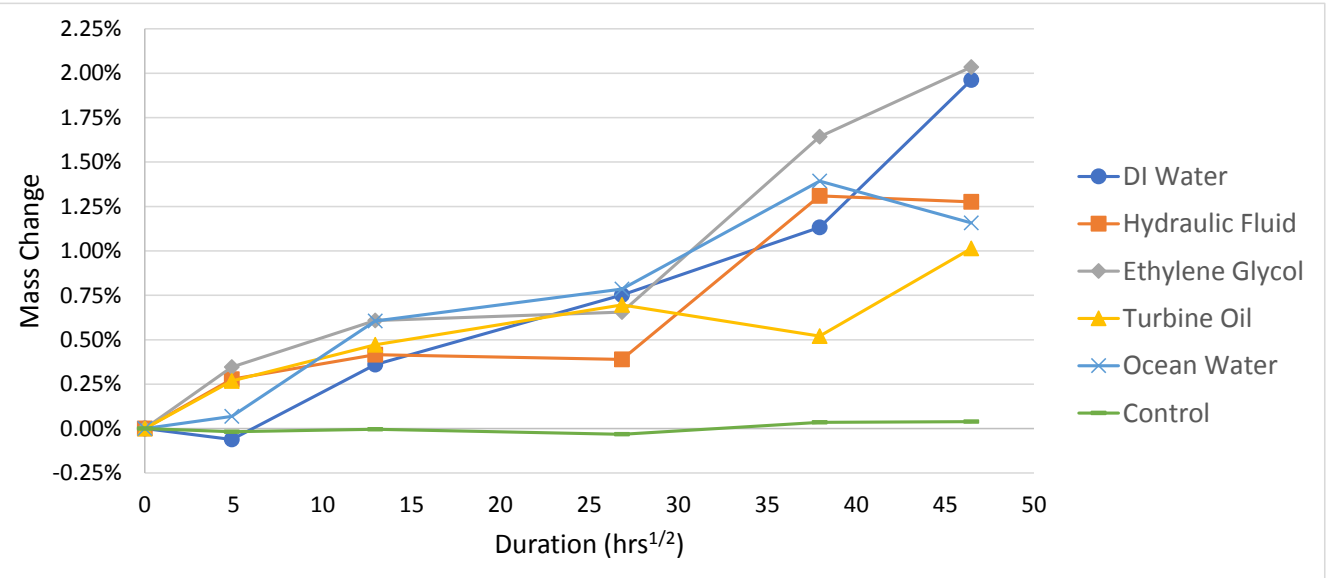

Figure 3.1: Change if mass of flexural samples as a function of $\sqrt{\text { hours }}$ of exposure.

Samples under investigation exhibited a net positive increase in moisture uptake as a function of exposure duration while control samples showed negligible mass change. Generally, the standard deviation of mass change for a sample group (a single liquid over a single duration) increased with increasing exposure duration, as shown in Table 3.3. Standard deviation of a sample group may be attributed to variations in local temperature, surface moisture removal, void distribution and void concentration.

Table 3.3: Standard deviation of percentage mass change, expressed in terms of duration and fluid.

\begin{tabular}{ccccccc}
\hline \multicolumn{6}{c}{ Standard Deviation of Mass Change } \\
\hline & DI & Hydraulic & Ethylene & Turbine & Ocean & Control \\
& Water & Fluid & Glycol & Oil & Water & \\
1 Day & $0.01 \%$ & $0.03 \%$ & $0.04 \%$ & $0.01 \%$ & $0.01 \%$ & $0.01 \%$ \\
1 Week & $0.09 \%$ & $0.06 \%$ & $0.20 \%$ & $0.02 \%$ & $0.08 \%$ & $0.01 \%$ \\
1 Month & $0.35 \%$ & $0.06 \%$ & $0.15 \%$ & $0.04 \%$ & $0.21 \%$ & $0.03 \%$ \\
2 Months & $0.10 \%$ & $0.11 \%$ & $0.20 \%$ & $0.04 \%$ & $0.17 \%$ & $0.03 \%$ \\
3 Months & $0.25 \%$ & $0.39 \%$ & $0.25 \%$ & $0.17 \%$ & $0.22 \%$ & $0.03 \%$ \\
\hline
\end{tabular}

A Fickian diffusion model predicts a linear region of mass increase followed by a plateau of moisture absorption associated with laminate saturation [102]. Moisture 
absorption values presented are strictly those of flexural samples after conditioning, prior to flexural testing, as short-duration sample mass measurements were halted after three weeks due to gasket moisture uptake (discussed in detail in Section 4.3). This resulted in a lack of a consistent reference measurement period. Hence, rigorous determination of the saturation moisture content through comparison of absorption over subsequent reference periods is not possible. However, as no plateaus were observed in the mass change vs. $\sqrt{\text { hours }}$ curves, it can be deduced that the samples did not reach moisture saturation over three months of exposure. To fit the obtained results to a Fickian model, saturation values of moisture absorption from the second campaign will be used and discussed accordingly. This, however, requires the assumption that an equivalent saturation moisture content is achieved despite the disparity in void content.

A three month duration was selected as literature indicated that FRPMCs, of comparable stacking sequence and make-up to that of the material under investigation, could achieve moisture saturation in less than three months at equivalent or lower conditioning temperatures $[32,37,64,66]$. This result brought to attention the differences in moisture absorptions rates and, consequently, the resulting time to achieve saturation, of samples conditioned through traditional immersion compared to that of single-sided exposure. Through a combination of an increased surface area to volume ratio and edge effects brought about by exposed laminate edges [103], complete sample immersion allows for an accelerated rate of absorption unachievable for conventional, in-service composite laminates.

While limited data points made it difficult to confirm linearity, the obtained data can be used to approximate linear absorption rates. Table 3.4 presents rates of moisture absorption, approximated as the slope of a linear regression through the origin of percentage mass change as a function $\sqrt{\text { hours }}$ of exposure curves. 
Table 3.4: Rates of change of percentage moisture absorption.

\begin{tabular}{ccccccc} 
& DI Water & Hydraulic Fluid & Ethylene Glycol & Turbine Oil & Ocean Water & Control \\
\hline$\frac{\Delta M}{\Delta \sqrt{t}}\left(\frac{\%}{\sqrt{h r s}}\right)$ & 0.0352 & 0.0280 & 0.0408 & 0.0206 & 0.0301 & 0.0005
\end{tabular}

It is hypothesized that the greater absorption rate of observed for ethylene glycol, DI water and ocean water compared to hydraulic fluid and turbine oil is attributed to a combination of the relatively small molecular size and polarity of the former. These attributes promote ingress into smaller, nanoscopic apertures through favourable, hydrophilic interactions between the absorbed solvent and epoxy's polar functional groups. The decrease in mass of DI water samples after a single day of conditioning may be attributed to the elution of unbound matrix material from the sample's surface which was then removed through the wiping procedure applied to remove surface moisture. A similar decrease in mass was not observed for ocean water samples of the same conditioning duration, likely due to mass gained from sub-surface salt precipitation.

\subsubsection{Sample Metrology}

Table 3.5 presents the two-factor ANOVA performed on the metrological data obtained from flexural samples following their designated exposure duration. Here, response variables - length, width and thickness - have been compared against the experiment's independent variables - chemical type and exposure time - to determine the statistical significance of independent variables on the experiment's outcome. Selecting $p=0.01$ as a cutoff value, it can be seen that chemical type, exposure duration nor their interaction demonstrated a statistically significant effect on either the percentage change in length or width of the sample. However, there is a statistically significant effect of chemical type, exposure duration and their interaction on the 
percentage change in thickness.

It can be hypothesized that this result is the consequence of one three phenomena: the quasi-isotropic nature of length and width directions in contrast to their anisotropy with the thickness direction, a gradient in moisture content through the thickness, or the difference in boundary conditions imposed by the conditioning fixture. In the first case, dissimilarity between the linear swelling coefficients of epoxy and carbon-fibre inhibit swelling of epoxy in the length and width directions through interlaminar shear induced by its bond to the carbon-fibre [104]. Conversely, swelling of the epoxy in the thickness direction is unrestrained. In the second case, swelling felt in the layers at the composite-liquid barrier is hampered by interlaminar shear induced by resistance of lower, unexposed layers. In the final case, the greater malleability of the silicon gasket material may have allowed for the samples to increase in thickness while the rigidity of PTFE hindered length and width expansion. These hypotheses will seek validation through SBS metrology, which will allow samples to swell, unrestrained.

\subsubsection{Flexural Properties}

Flexural testing was performed in accordance with ASTM D7264 [86]. Table 3.6 outlines the specifications of ASTM D7264 and any required deviations. Changes made were driven by equipment and material availability.

Prior to conditioning, dried samples were tested to establish baseline flexural properties. Due to limited material availability, samples were cut both parallel and perpendicular to the fabric warp direction. As both the warp and weft are composed of yarn consisting of the same number of fibres they are visibly indistinguishable, hence fibre orientation will be referred to by the visible orientation of the fibres in the outermost plies. Figure 3.2 compares the visible fibre orientation in the outer 
Table 3.5: Two-factor ANOVA summary for percentage change of sample length, width and thickness; significant results are highlighted in bold.

\begin{tabular}{|c|c|c|c|c|c|}
\hline $\begin{array}{l}\text { Source of } \\
\text { Variation }\end{array}$ & $\begin{array}{l}\text { Sum of } \\
\text { Squares }\end{array}$ & $\begin{array}{c}\text { Degrees of } \\
\text { Freedom }\end{array}$ & $\begin{array}{c}\text { Mean } \\
\text { Square }\end{array}$ & $F_{0}$ & P-Value \\
\hline \multicolumn{6}{|c|}{ Length } \\
\hline Chemical & $2.88 \mathrm{E}-06$ & 5 & $5.75 \mathrm{E}-07$ & 2.1329 & 0.073668 \\
\hline Time & $3.54 \mathrm{E}-06$ & 4 & 8.85E-07 & 3.2814 & 0.016888 \\
\hline Interaction & $5.26 \mathrm{E}-06$ & 20 & $2.63 \mathrm{E}-07$ & 0.9747 & 0.503379 \\
\hline Error & $1.62 \mathrm{E}-05$ & 60 & $2.70 \mathrm{E}-07$ & & \\
\hline Total & $2.79 \mathrm{E}-05$ & 89 & & & \\
\hline \multicolumn{6}{|c|}{ Width } \\
\hline Chemical & $8.49 \mathrm{E}-05$ & 5 & $1.70 \mathrm{E}-05$ & 0.8225 & 0.538572 \\
\hline Time & $1.12 \mathrm{E}-04$ & 4 & $2.81 \mathrm{E}-05$ & 1.3587 & 0.259013 \\
\hline Interaction & $2.98 \mathrm{E}-04$ & 20 & $1.49 \mathrm{E}-05$ & 0.7207 & 0.789464 \\
\hline Error & $1.24 \mathrm{E}-03$ & 60 & $2.07 \mathrm{E}-0.5$ & & \\
\hline Total & $1.73 \mathrm{E}-03$ & 89 & & & \\
\hline \multicolumn{6}{|c|}{ Thickness } \\
\hline Chemical & $1.28 \mathrm{E}-03$ & 5 & $2.57 \mathrm{E}-04$ & 8.6908 & 0.000003 \\
\hline Time & $7.18 \mathrm{E}-04$ & 4 & $1.80 \mathrm{E}-04$ & 6.0752 & 0.000358 \\
\hline Interaction & $1.82 \mathrm{E}-03$ & 20 & $9.08 \mathrm{E}-05$ & 3.0719 & 0.000411 \\
\hline Error & $1.77 \mathrm{E}-03$ & 60 & $2.96 \mathrm{E}-05$ & & \\
\hline Total & $5.59 \mathrm{E}-03$ & 89 & & & \\
\hline
\end{tabular}


Table 3.6: Specified and utilized test parameters for ASTM D7264; deviations from the specified standard highlighted in bold.

\begin{tabular}{|l|l|l|}
\hline \multicolumn{1}{|c|}{ Test Parameter } & \multicolumn{1}{|c|}{ Specification } & \multicolumn{1}{c|}{ As Tested } \\
\hline $\begin{array}{l}\text { Loading nose } \\
\text { and supports shape }\end{array}$ & $\begin{array}{l}\text { smooth, cylindrical, } \\
5.0 \pm 1.0 \mathrm{~mm} \text { radii }\end{array}$ & $\begin{array}{l}\text { smooth, cylindrical, } \\
5.0 \pm 1.0 \mathrm{~mm} \text { radii }\end{array}$ \\
\hline $\begin{array}{l}\text { Loading nose } \\
\text { and supports hardness }\end{array}$ & $\geq 55 \mathrm{HRC}$ & $>55 \mathrm{HRC}$ \\
\hline $\begin{array}{l}\text { Loading nose } \\
\text { and supports type }\end{array}$ & Fixed, rotatable or rolling & Rolling \\
\hline $\begin{array}{l}\text { Width and thickness } \\
\text { metrology }\end{array}$ & $\begin{array}{l}\text { Micrometer, } 4-7 \mathrm{~mm} \text { ball- } \\
\text { interface, } \pm 0.02 \mathrm{~mm}\end{array}$ & $\begin{array}{l}\text { Caliper, } \\
\pm \mathbf{0 . 0 1} \text { mm }\end{array}$ \\
\hline $\begin{array}{l}\text { Length metrology } \\
\text { or caliper } \pm 0.1 \mathrm{~mm}\end{array}$ & $\begin{array}{l}\text { Caliper, } \\
\pm 0.01 \mathrm{~mm}\end{array}$ \\
\hline $\begin{array}{l}\text { Deflection } \\
\text { measurement }\end{array}$ & Transducer, mid-span & $\begin{array}{l}\text { Axial extensometer, } \\
\text { fixture-mounted }\end{array}$ \\
\hline Width & $13 \mathrm{~mm}$ & 0.5 in \\
\hline Span-to-thickness & $32: 1$ & $32: 1$ (4 in : 0.125 in) \\
\hline Number of replicates & five & three \\
\hline Crosshead speed & $1 \mathrm{~mm} / \mathrm{min}$ & $1 \mathrm{~mm} / \mathrm{min}$ \\
\hline Span overhang & $20 \%$ & $20 \%$ \\
\hline
\end{tabular}




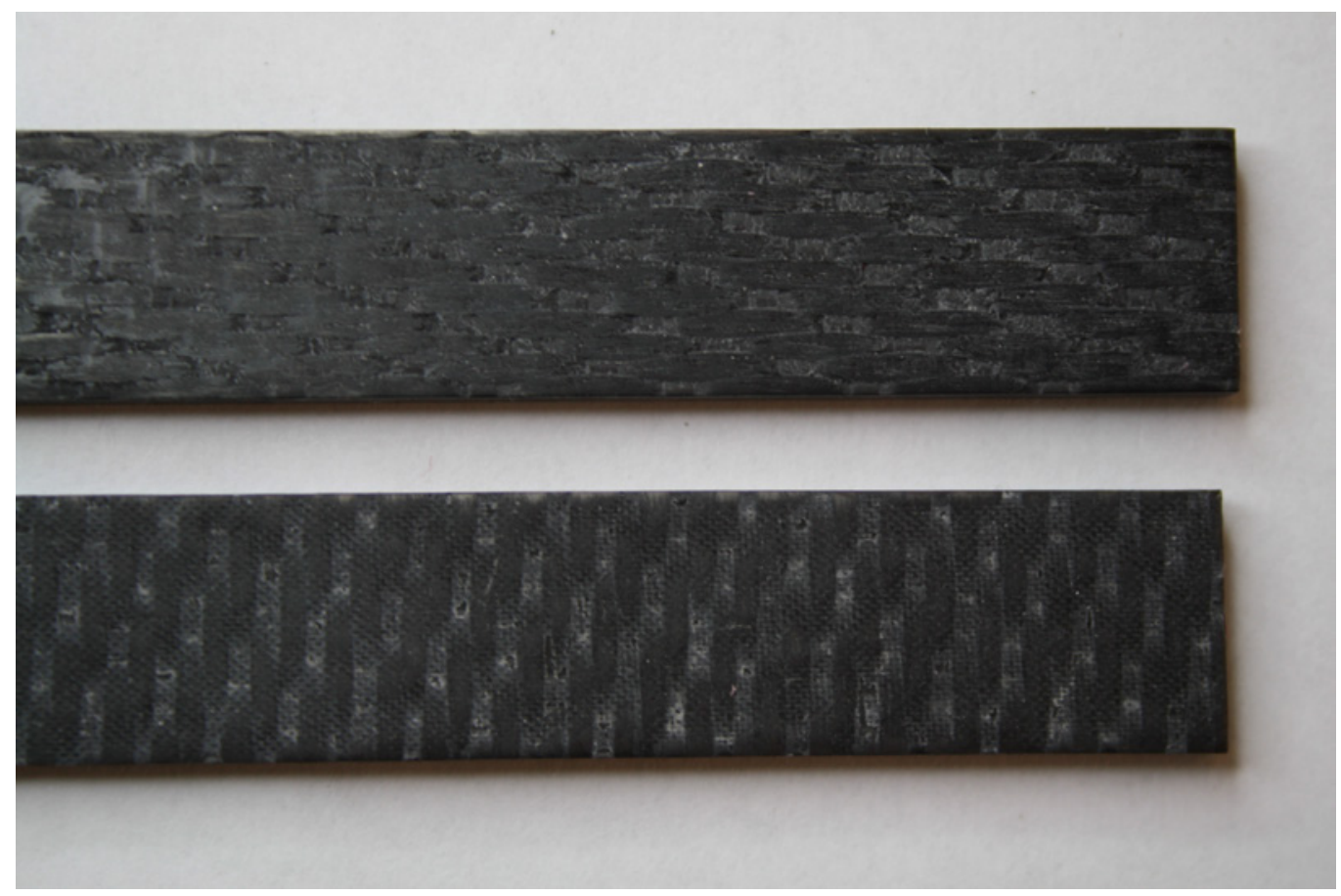

Figure 3.2: Two flexural samples illustrating a tool-side with parallel fibres (top) and bag-side with perpendicular fibres (bottom).

plies of two flexural samples. Due the orthogonal nature of warp and weft yarns in an 8-harness weave, all samples possess both a parallel and perpendicular surface. Thus, to establish convention, samples will be referred to by the visible fibres on their bag-side surface. Samples which possess a bag-side surface with visible fibre running parallel to specimen length will be referred to as 'parallel' and those which possess fibres running perpendicular to specimen length will be referred to as 'perpendicular'. Due to the relative variability of the bag-side surface - a result of inconsistencies at the bagging surface during curing - it was decided that all flexural tests will be performed with the bag-side surface in compression to elicit results from a worst-case flexural scenario.

Figure 3.3 displays the stress-strain curve for 14 baseline flexural samples tested. It is important to note that all baseline samples failed due to buckling in their compressive surface, at or near the loading nose. As can be see, while samples exhibited a 


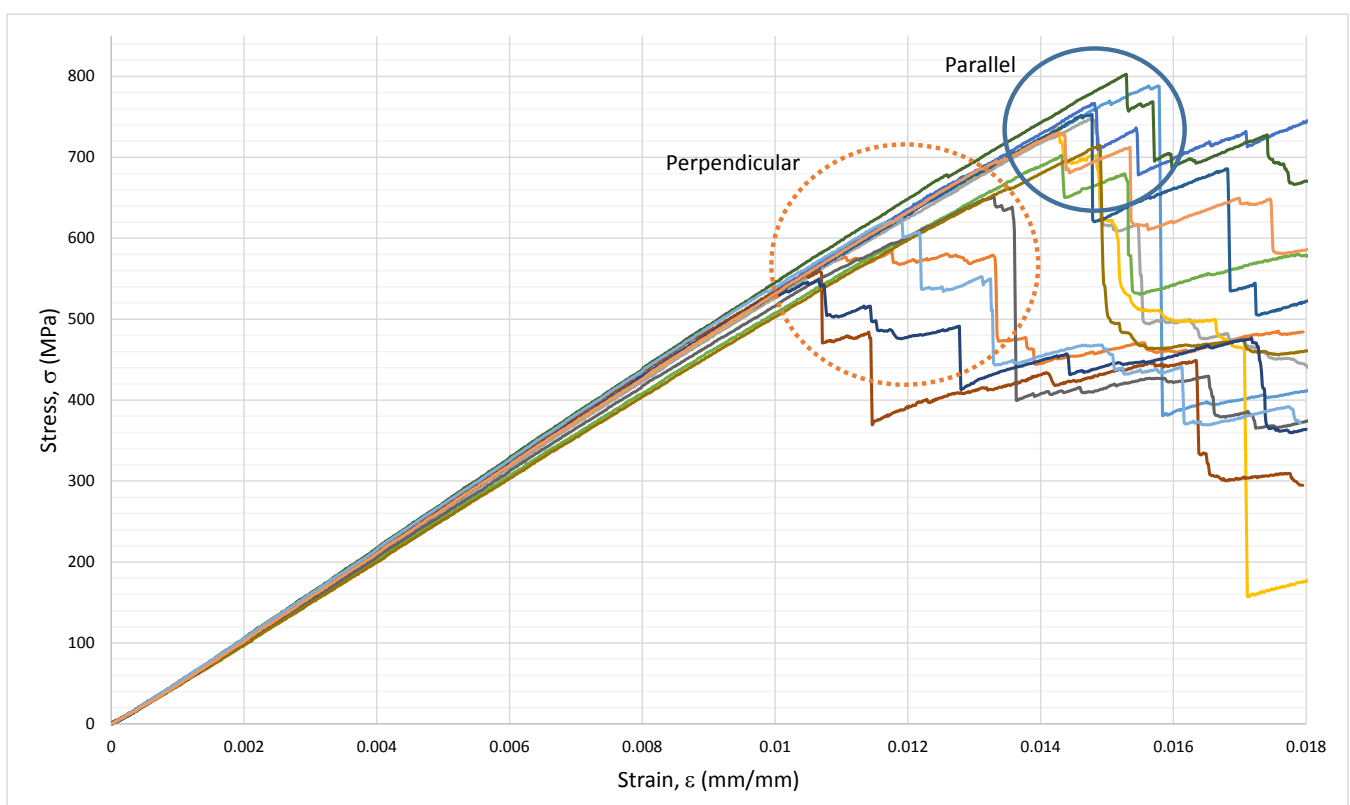

Figure 3.3: Stress-strain curves of unconditioned samples; failure of samples with top-surface fibres parallel and perpendicular to sample length.

narrow distribution of flexural moduli, there is a clear bimodal distribution of flexural strengths correlated with the visible fibre orientation in the bag-side surfaceperpendicular samples exhibiting a lower maximum flexural strength than parallel samples. Table 3.7 presents accompanying flexural and metrological data for baseline flexural samples. To address this disparity, it was decided that fibre orientation would be consistent for a given exposure duration, i.e. all samples conditioned for one day would have a parallel fibre orientation. Hence, one-day and one-week samples possess a parallel orientation while one-month, two-month and three-month samples possess a perpendicular orientation, the designation of which was arbitrary, governed only by the total number of parallel and perpendicular samples obtained. To properly compare sample groups of differing fibre orientations, results have being normalized to their respective baseline flexural strengths.

Figures 3.4 and 3.5 summarize flexural data obtained for all conditioned samples with 
Table 3.7: Flexural and metrological data for baseline flexural samples.

\begin{tabular}{|c|c|c|c|c|c|c|}
\hline & $\begin{array}{c}\text { Maximum Flexural } \\
\text { Strength }\end{array}$ & $\begin{array}{l}\text { Modulus of } \\
\text { Elasticity }\end{array}$ & Mass & Thickness & Length & Width \\
\hline & $M P a$ & $G P a$ & $g$ & in & in & in \\
\hline \multicolumn{7}{|c|}{ Parallel Alignment } \\
\hline Average & 744.16 & 53.28 & 7.2821 & 0.125 & 4.763 & 0.498 \\
\hline $\begin{array}{l}\text { Standard } \\
\text { Deviation }\end{array}$ & 29.52 & 1.33 & 0.0890 & 0.001 & 0.021 & 0.010 \\
\hline $\mathrm{CV}$ & $3.97 \%$ & $2.50 \%$ & $0.79 \%$ & $0.73 \%$ & $0.44 \%$ & $2.10 \%$ \\
\hline \multicolumn{7}{|c|}{ Perpendicular Alignment } \\
\hline Average & 593.57 & 54.15 & 7.3107 & 0.125 & 4.794 & 0.498 \\
\hline $\begin{array}{l}\text { Standard } \\
\text { Deviation }\end{array}$ & 43.75 & 0.87 & 0.1163 & 0.001 & 0.024 & 0.008 \\
\hline $\mathrm{CV}$ & $7.37 \%$ & $1.61 \%$ & $1.35 \%$ & $0.87 \%$ & $0.50 \%$ & $1.56 \%$ \\
\hline
\end{tabular}

each column representing the average, normalized maximum flexural strength (flexural strength) or flexural modulus of elasticity (flexural modulus) of its respective sample group. Dashed-lines about the one value represent the standard deviation of baseline perpendicular samples while continuous lines represent the standard deviation of baseline parallel samples.

Flexural strength results demonstrated a wider distribution of values for each sample group when compared to the values of flexural modulus. As a matrix-dominated parameter, the flexural strength is highly dependent on void content. Hence, large standard deviation may be attributed to the laminate's observed high void content. Similarly, changes to the flexural moduli were less substantial than changes to flexural strengths as the observed degradation is attributed to chemical changes to the epoxy matrix. Micro-stresses brought about by irregularity in the bag-side compression surface may also have contributed to mechanical variability. 


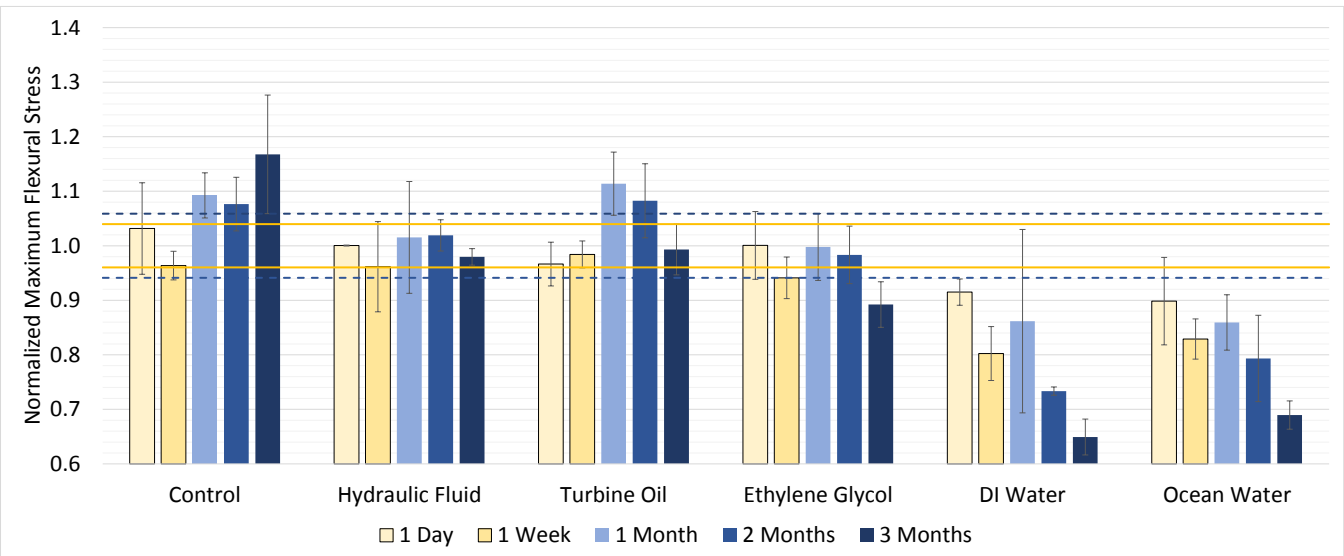

Figure 3.4: Normalized flexural strengths as a function of conditioning liquid and duration.

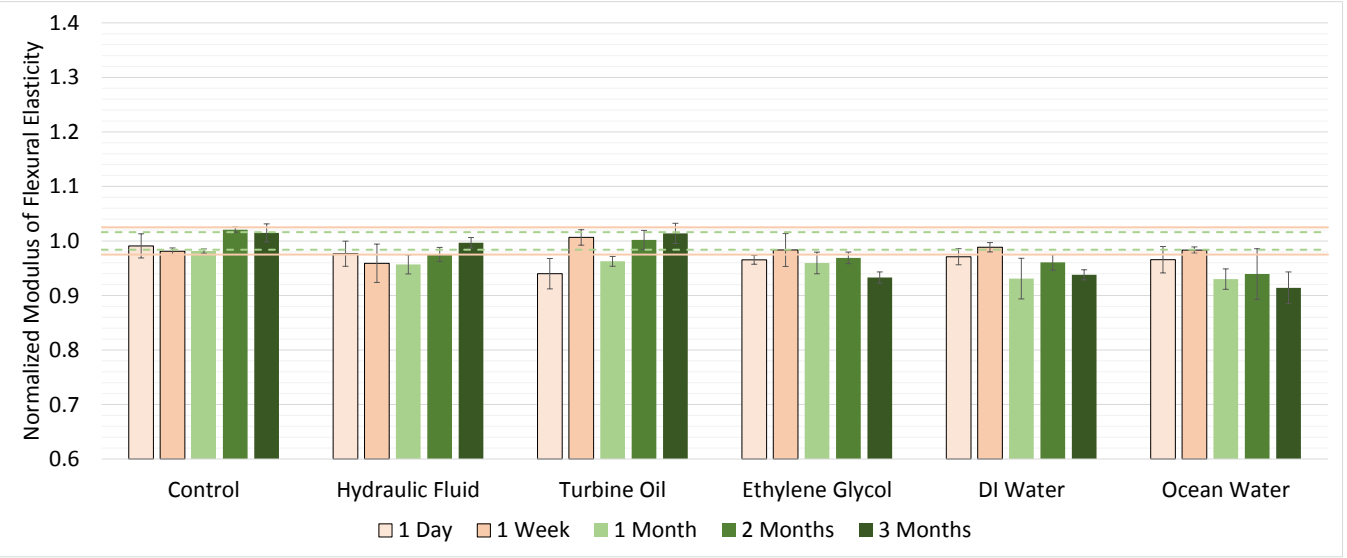

Figure 3.5: Normalized flexural moduli as a function of conditioning liquid and duration. 
Observed trends in flexural strengths mirrored those of flexural moduli: with increasing duration control samples showed a net increase in flexural properties; ethylene glycol, DI water and ocean water samples showed a net decrease in flexural properties; and hydraulic fluid and turbine oil samples showed a negligible change in flexural properties. Table 3.8 presents the two-factor ANOVA performed considering flexural strength and modulus as response variables. P-values, significantly below the cutoff value of $p=0.01$ show a strong statistical correlation between independent variables and response variables, with chemical type showing the most appreciable effects. These results call for rejection of the null hypothesis and justify further, post hoc analysis.

Table 3.8: Two-factor ANOVA summary for change in flexural strength and modulus.

\begin{tabular}{cccccc}
\hline $\begin{array}{c}\text { Source of } \\
\text { Variation }\end{array}$ & $\begin{array}{c}\text { Sum of } \\
\text { Squares }\end{array}$ & $\begin{array}{c}\text { Degrees of } \\
\text { Freedom }\end{array}$ & $\begin{array}{c}\text { Mean } \\
\text { Square }\end{array}$ & $\boldsymbol{F}_{\mathbf{0}}$ & P-value \\
\hline \multicolumn{5}{c}{ Flexural Strength } \\
\hline Chemical & 0.8713 & 5 & 0.1743 & 44.3718 & $\mathbf{6 . 4 8 5 E - 1 9}$ \\
Duration & 0.1076 & 4 & 0.0269 & 6.8492 & $\mathbf{1 . 3 0 9 E - 0 4}$ \\
Interaction & 0.1962 & 20 & 0.0098 & 2.4983 & $\mathbf{3 . 3 2 6 E - 0 3}$ \\
Error & 0.2356 & 60 & 0.0039 & & \\
Total & 1.4108 & 89 & & & \\
\hline \multirow{2}{*}{$\begin{array}{c}\text { Clexural Modulus } \\
\text { Chemical }\end{array}$} & 0.0204 & 5 & 0.0041 & 9.5715 & $\mathbf{9 . 4 2 6 E - 0 7}$ \\
Duration & 0.0055 & 4 & 0.0014 & 3.2352 & $\mathbf{1 . 8 0 4 E - 0 2}$ \\
Interaction & 0.0269 & 20 & 0.0013 & 3.1534 & $\mathbf{3 . 0 6 3 E - 0 4}$ \\
Error & 0.0256 & 60 & 0.0004 & & \\
\hline Total & 0.0785 & 89 & & & \\
\hline
\end{tabular}

Applying Dunnett's test, assuming all sample groups exhibited a normal distribution, 
using a two-tailed approach, with a $99 \%$ confidence interval, both DI and ocean water demonstrated a statistically significant decrease in flexural strength following one week, two months and three months of conditioning. However, application of Tukey's test using the same parameters, demonstrated there to be no statistical significance between DI and ocean water sample groups compared at the same duration. This indicates that the aqueous salts dissolved in the ocean water did not have an appreciable contribution to degradation - either by increasing ingress rates or chemical reaction - as supported by literature investigating similar materials [36,55]. Notably, DI and ocean water samples showed an appreciable (8.5\% and $10.2 \%$, respectively) degradation after a single day and substantial degradation (35.1\% and 31.1\%, respectively) after three months of exposure. Similar analysis of control, hydraulic fluid, turbine oil, and ethylene glycol samples did not show a significant change in flexural strength, forcing the acceptance of the null hypothesis: The exposing chemical did not cause a change in the tested composite's flexural strength. The observed increase in flexural strength of control samples may be the result of a long term post-cure which demonstrates significance over longer durations. Likewise, based on the observed trend, decrease in flexural strength of ethylene glycol exposed samples may prove significant over longer exposure periods.

Similar statistical analysis of flexural moduli demonstrated that turbine oil and hydraulic fluid caused a significant decrease over one day and one month, respectively. However, because these results are not part of identifiable trend, this is believed to be an anomalous result. Ocean water showed a statistically significant decrease in moduli at and above one month of exposure, while DI water showed significance only for one month and three month samples. Ethylene glycol demonstrated significance after three months of exposure. Again, Tukey's test revealed no statistical significance between DI and ocean water sample groups compared at the same duration. Table 
3.9 provides a summary of post hoc statistical analysis performed. Noted anomalous data from the one-month sample groups demonstrated greater than anticipate flexural strength and lower than anticipate flexural moduli. Hence, it is believed that non-material specific differences resulted from either variability in one-month sample conditioning or one-month sample flexural testing, although explicit causality is unclear. 


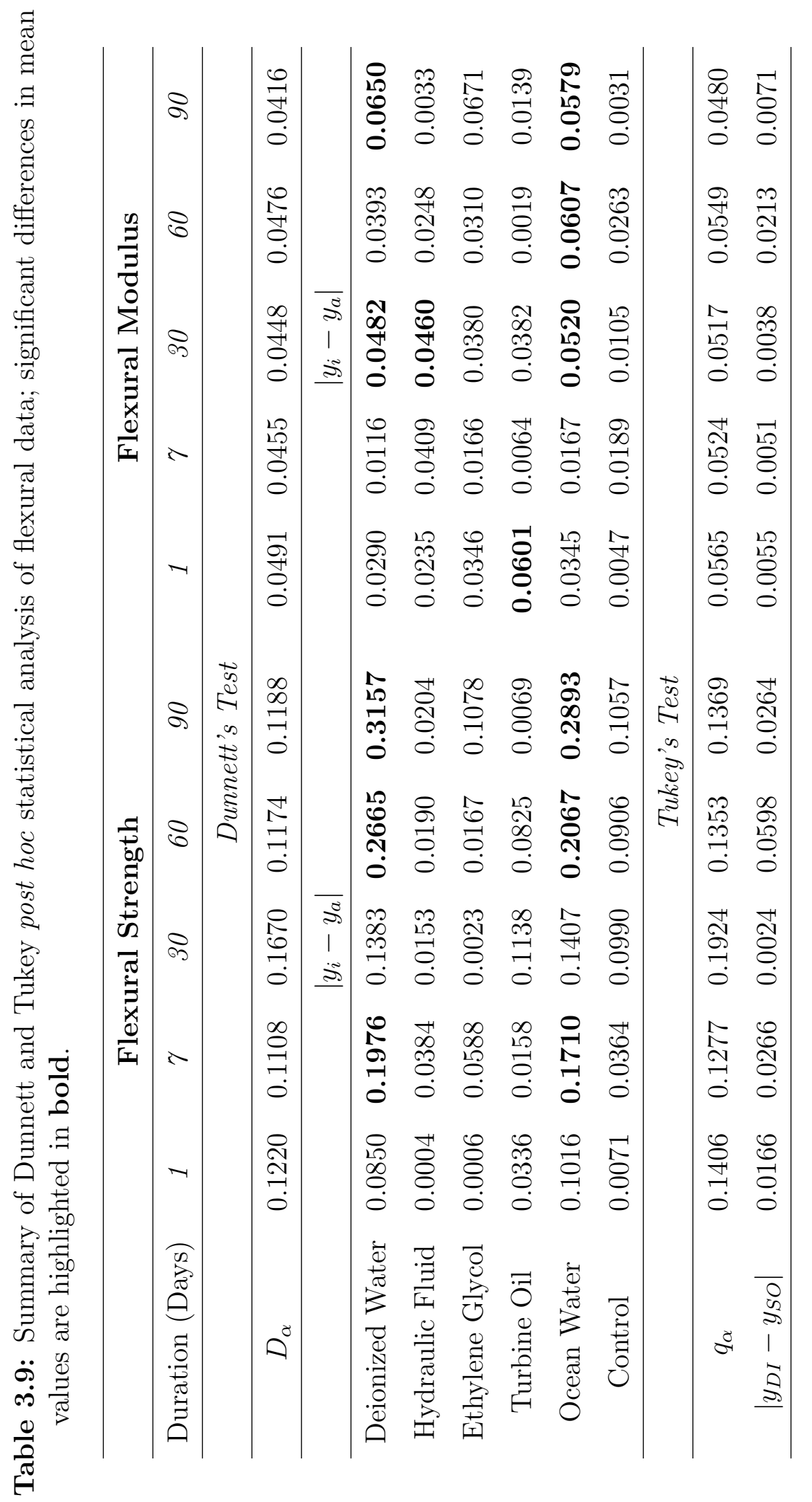




\subsubsection{Chemical Considerations}

Figure 3.6 compares changes in the material's flexural strength as a function of moisture absorption. Linear regression of the data shows that hydraulic fluid and turbine oil do not exhibit a correlation between liquid uptake and decrement of flexural strength, however, there is an association between uptake of DI water, ocean water and ethylene glycol and a decrease in flexural strength. Such a result demonstrated that, although carbon-fibre reinforced epoxy has the propensity to physically absorb both polar and nonpolar liquids, there is a unique, degradative interaction between investigated polar solvents and the epoxy matrix.

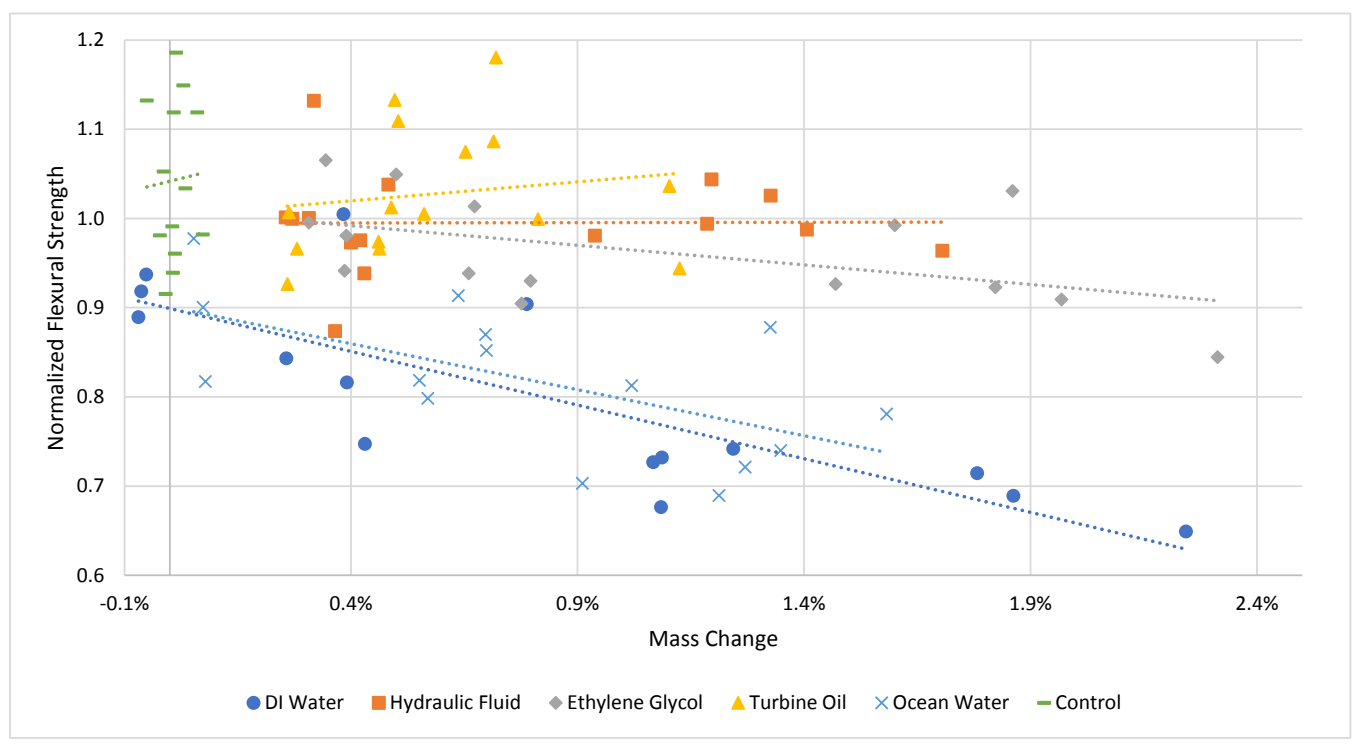

Figure 3.6: Normalized flexural strength as a function of percentage change in mass resulting from fluid uptake.

While the exact composition of AX-5180 is unknown, inferences can be made regarding its known components. The matrix of AX-5180 prepreg is composed of seven ingredients; an epoxy resin copolymer, an epoxy curing agent and a flame retardantwhich are proprietary - poly(bisphenol A-co-epichlorohydrin) (also known as bisphenol A diglycidyl ether or DGEBA), poly[(phenyl glycidyl ether)-co-formaldehyde] 
(PPGE), fumed silica and cyanoguanidine [1]. Figure 3.7 displays the molecular structures of known resin components. Cyanoguanidine is a latent hardener which reacts with linear, uncured epoxy resin, such as linear epoxy oligomers PPGE and DGEBA, to form a three dimensional, crosslinked thermoset [105]. Fumed silica, naturally occurring as quartz and a common component of most glasses, is a filler materials sometimes added to epoxy as a thickening agent [106].

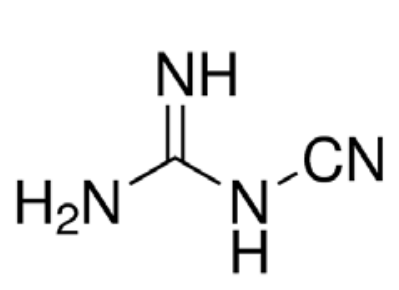

(i)

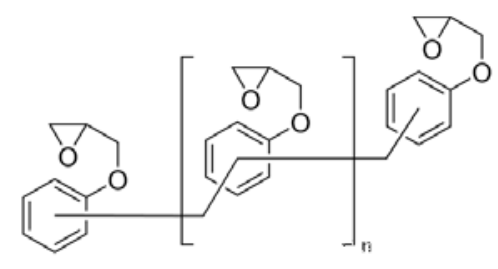

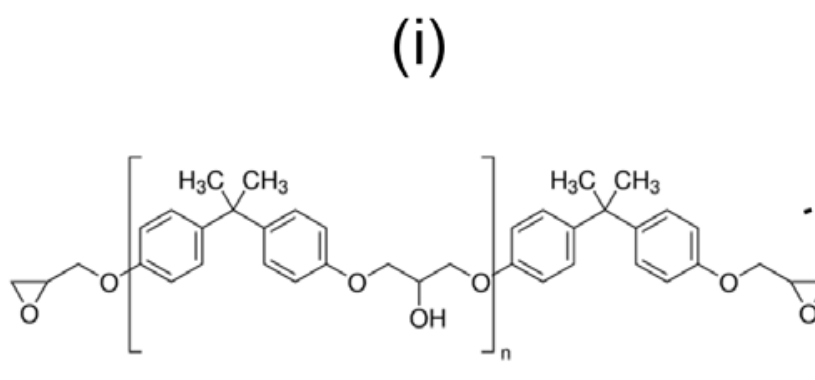

(iii) (ii)

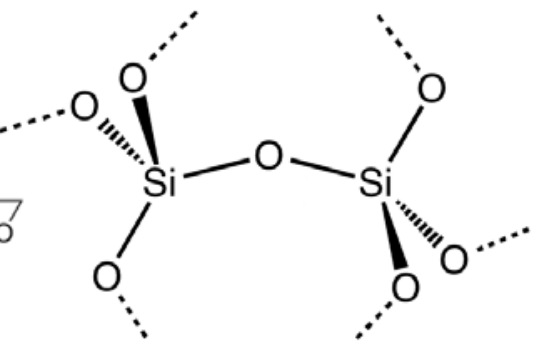

(iv)

Figure 3.7: Molecular structures of i) cyanoguanidine, ii) poly[(phenyl glycidyl ether)-co-formaldehyde], iii) poly(bisphenol A-co-epichlorohydrin), glycidyl end-capped and iv) fumed silica.

A number of sources discuss the curing reaction of DGEBA with a tetra-functional amine hardener $[7,105]$. Summarized here for brevity, amine functional groups of the hardener react with the oxirane groups of DGEBA through a ring-opening reaction forming a covalent bond and a secondary hydroxyl group on the DGEBA backbone. The tetra-functional hardener, now with three available amines, reacts with three additional DGEBA oligomers. This reaction, taking place at both DGEBA oxirane groups, forms a three dimensional networks between the DGEBA backbone and 
amine crosslinker, forming the final cured thermoset polymer. Figure 3.8 illustrates a crosslinked DGEBA with $R$ groups representing the nonreactive central moiety of the amine hardener. While a single macromolecular network can theoretically be formed, realistically, mass transport limits the extent of reaction, forming multiple interlaced networks, each with unreacted amine and oxirane groups [7]. Similarly, while secondary hydroxyl group on the DGEBA backbone have the propensity to react, they are typically limited by steric hindrance. Hence, unreacted hydroxyl and amine functional groups, which have a certain degree of steric freedom, are capable of taking part in intermolecular interactions. The degree of crosslinking and intermolecular interactions dictates an epoxy's mechanical properties. While AX-5180 contains an epoxy resin copolymer and epoxy curing agent for which the molecular structure is unknown, it can be speculated that these compounds are of a similar structure to the backbone and hardener elements discussed and are added to dictate the degree of crosslinking. Alternatively, the curing agent may possess some form of catalyst used to control rate of cure which does not contribute to the thermoset structure, although this is speculation.

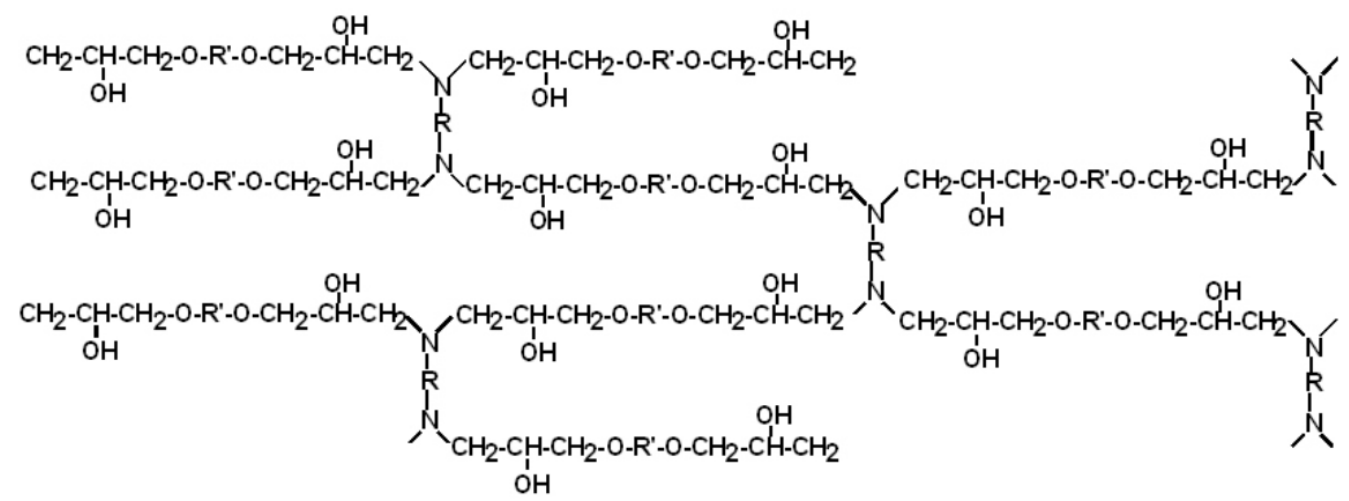

Figure 3.8: Molecular structure of a crosslinked DGEBA thermoset polymer, adapted from [7].

Comparing the obtained flexural results and the molecular structures of the tested 
liquids, it can be concluded that the degradation of the epoxy matrix is brought about by hydroxyl group interactions between the ingressed liquids and hydrogen bonds of the epoxy matrix. Figure 3.9 displays the molecular structures of water, ethylene glycol, and 1-decene - the primary component of Royco 782 hydraulic fluid [107]. It should be noted that 1-decene is present in Royco 782 as both a tetramer and a trimer, not its monomeric form. While the bulk composition of Aeroshell-Turbine Oil 390 is not presented in literature, as a synthetic lubricant, it is anticipated to have a similar linear long-chain hydrocarbon structure to that of 1-decene.

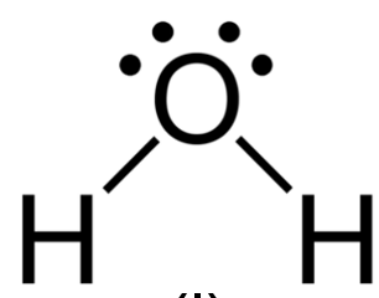

(i)

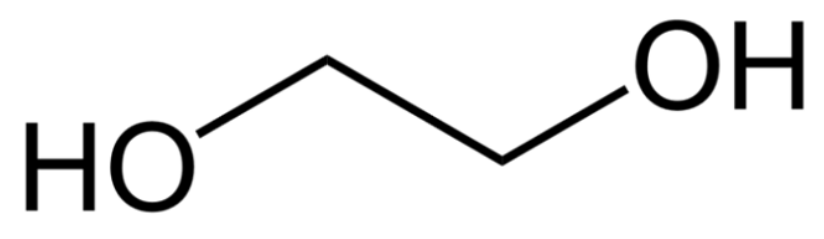

(ii)

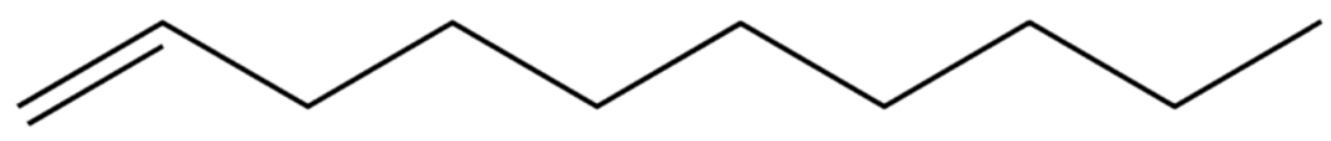

(iii)

Figure 3.9: Molecular structures of i) water, ii) ethylene glycol and iii) 1-decene.

Supported by literature [7,108,109], water disturbs intermolecular hydrogen bonding of interacting hydroxyl and amine groups of the epoxy matrix, lowering matrixdominate mechanical properties. This process may be reversible, contingent on the removal of ingressed liquid (i.e. through evaporation). As a protic solvent possessing a relatively high polarity (a dielectric constant of 37.7 [110]) it is anticipated that ethylene glycol may be capable of similar intermolecular disruptions, attributing to the observed long-term decrease in flexural properties. Conversely, nonpolar longchain hydrocarbons such as 1-decene, by design, are incapable of similar dipole-dipole interactions. 
With longer duration exposures, DI water sample wells exhibited a cloudiness resulting from the formation of white precipitate. This observation would indicate that either water is transporting and allowing for the agglomeration of unbound components within the composite or has the ability to disrupt and cleave intramolecular, bonds freeing epoxy or hardener subunits. Leaching of the constituent matrix has been evaluated through a drying study performed in conjunction with subsequent SBS testing and has been discussed in Section 3.2.1.

Inducing such a process of matrix leaching, subunit migration, subunit re-deposition and/or removal may lead to accelerated, irreversible changes to the matrix structure beyond that of static exposure to moisture, the evaluation of which is paramount based on the cyclic nature of aircraft environmental exposure. Literature investigating the effects of cyclic exposure of epoxy matrix composites to condensation and ultraviolet radiation has demonstrated a synergistic degradation brought about by cyclic exposure [32]. Hence, these observations call for further work to identify the molecular structure of the observed precipitate and to investigate the effects of longterm cyclic exposure of epoxy matrix composites to water-based solvents on flexural properties. Furthermore, validation of results should be performed through the exposure of an epoxy, FRPMC to a comparable, short chain, protic solvent, such a propanol or butanol and contrasted with highly polar protic solvent such as dimethyl sulfoxide. Analysis of flexural properties has demonstrated that OEM qualification testing can be confined to water based conditioning for regular internal, epoxy based FRPMC applications. This practice can effectively characterize the most common and most detrimental contaminant while offering cost-savings through the reduced material and labour requirements of a more focused test campaign. 


\subsubsection{Surface Microscopy}

Figure 3.10 shows optical microscopy performed on sample surfaces following direct fluid exposure for three months. Microscope images were captured under identical lighting conditions, subject to 3.5x magnification, using an Olympus SZH-10 stereo microscope. All samples were directly exposed to liquid on their bag-side; what are observed as small square tessellations is the impression of the plain-weave nylon peal-ply used to extract the composite laminate following curing. All three-month conditioned samples exhibited a distinctive yellow tinge, most notably for ethylene glycol samples, for which colour change was noted after as early as one week. Interestingly, conditioning ethylene glycol displayed a transition from colourless to yellow which increased in intensity with increasing exposure duration. Subsequent testing revealed that this appearance of colour was correlated with ethylene glycol's exposure to the composite sample and not do to leaching of the sample array's constituent components, nor elevated temperature exposure alone. Further chemical analysis is required to establish the cause of this colour change.
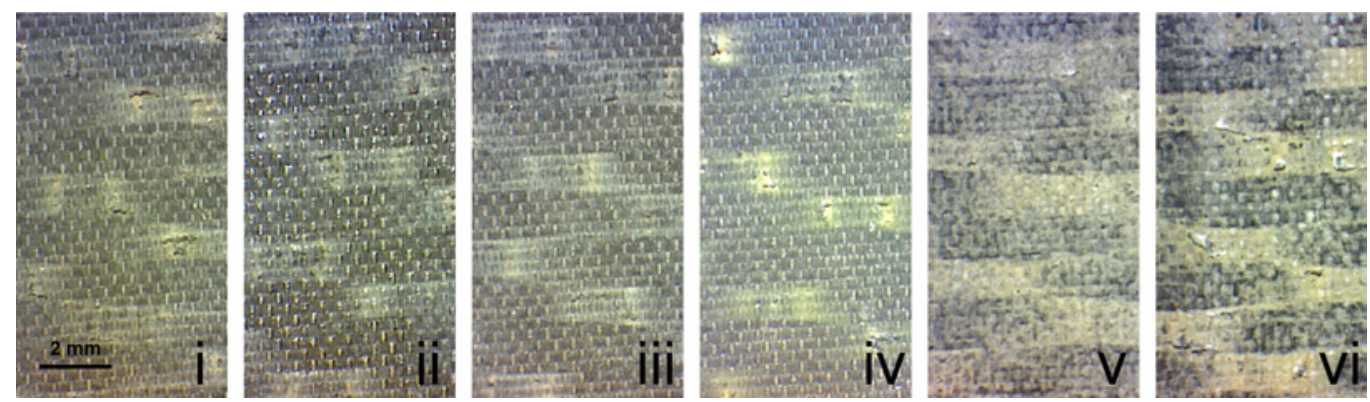

Figure 3.10: Surface structures of samples exposed to i) air (control), ii) hydraulic fluid, iii) turbine oil, iv) ethylene glycol, v) ocean water and vi) deionized water after a three-month conditioning duration.

The control, hydraulic fluid and turbine oil samples exhibited the same degree of surface void formation with a visually indistinguishable variation in size and distribution. Conversely, DI water samples demonstrated considerable surface degradation, with 
large voids evident in the surface epoxy. Sheen, a characteristic of light reflecting from the fibre surface, is visible in larger voids, illustrating degradation down to the top fibre layer. Figure 3.11 shows changes in surface degradation as a function of exposure duration for DI water samples. Increasing exposure duration demonstrated that a clear correlation to surface degradation which is more apparent in DI water samples than for the other liquids investigated. The unconditioned sample surface exhibits a representative degree of void formation inherent to the curing process, as well as the composite's innate, black colour, as prescribed by the supplier. Notably, after one-day DI water exposure, sample surfaces exhibited a stark contrast between bevelled regions in the top-ply where more epoxy could collect during impregnation and regions where top-ply fibres were closer to the vacuum bag and thus, are coated in a thinner layer of epoxy. Thicker epoxy regions transition from black to white, with increased void formation more prominent in resin-rich regions. Voids in ethylene glycol samples were larger and appeared in a greater concentration than those observed in hydraulic fluid or turbine oil samples but were less prevalent than those observed in DI water samples. Ocean water samples exhibited a coating of salt precipitate on the exposed surface. While voids were visible, the precipitate largely masks visibility of the sample surface and thus, hides possible degradation of surface epoxy. A comprehensive comparison of microscopy of the bag-side surface of each sample group is presented in Figures D.2 and D.3 in Appendix D.
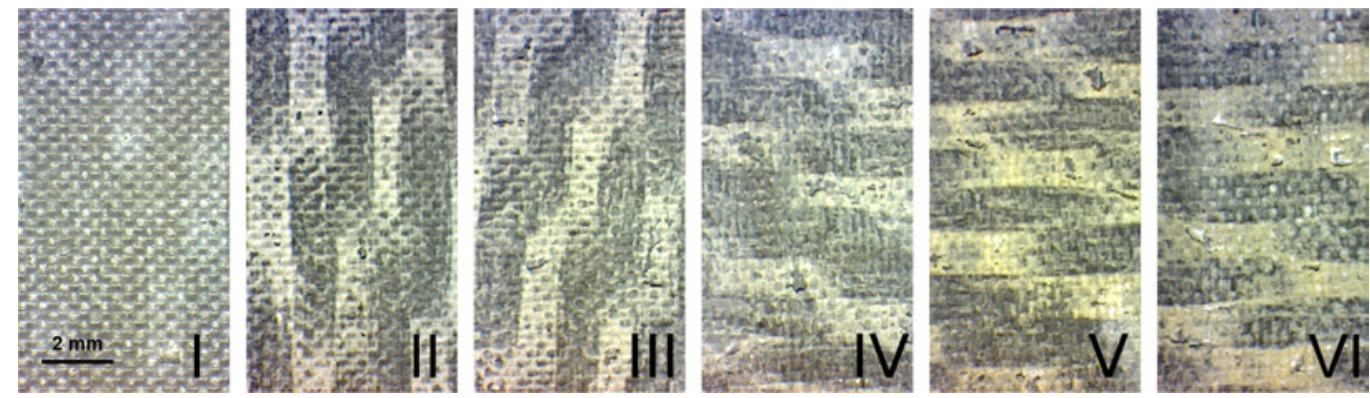

Figure 3.11: Surface structures of deionized water samples conditioned for I) 0, II) 24, III) 168, IV) 720, V) 1440 and VI) 2160 hours. 
Figures 3.12 and 3.13 compare an i) unconditioned and ii) three-month water sample, illustrating cross sectional and tool-side surfaces, respectively. Similar observation to those made for the bag-side can be made for both cross sections and tool-sides; epoxy-rich regions are whiter, more distinctive from the surrounding fibres and show a greater concentration of voids in the water exposed sample than in the unconditioned sample. This result indicates that moisture, initially confined to contact with the bagside surface, penetrated the sample's surface and permeated through the thickness, interacting with sub-surface epoxy.
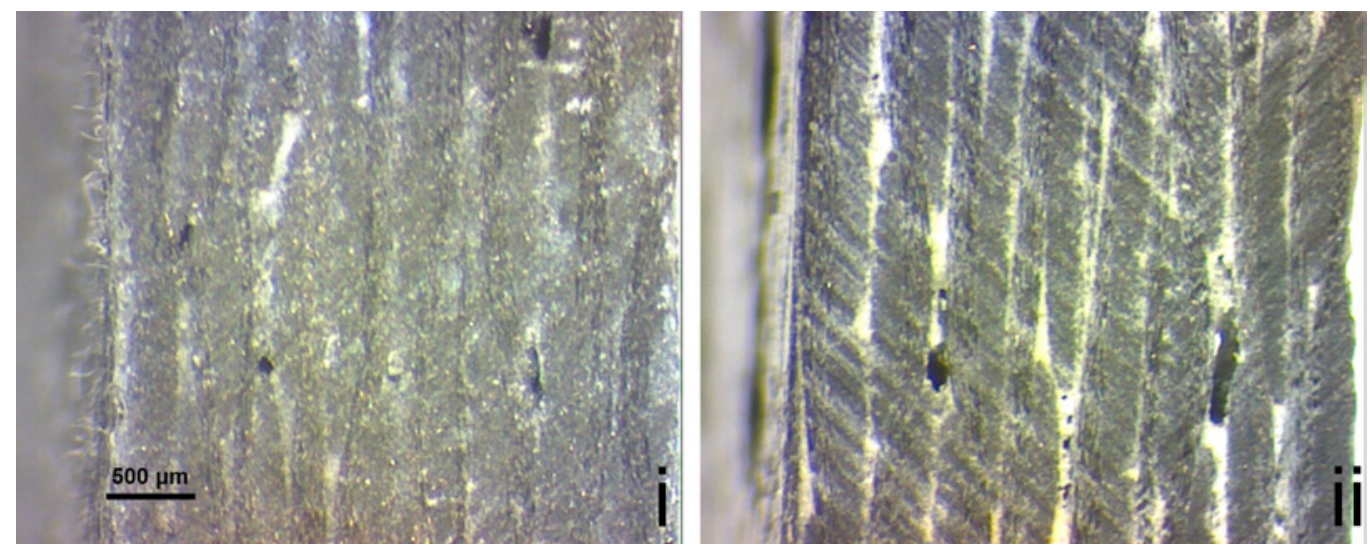

Figure 3.12: 12.5x magnification of the cross section of an i) unconditioned and ii) three-month DI water sample.
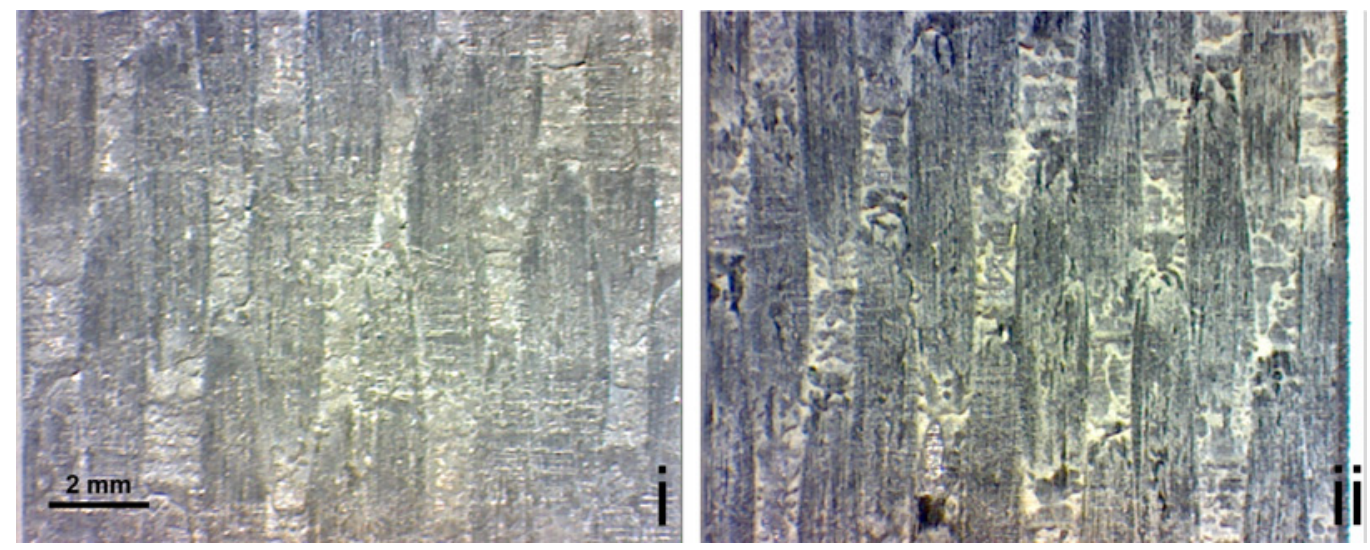

Figure 3.13: Unexposed tool-side surface of an i) unconditioned and ii) three-month DI water sample. 


\subsubsection{X-Ray Micro-computed Tomography}

X-ray micro-computed tomography was performed in order to assess the internal structure of flexural samples. To ensure changes in structure (i.e. voids and delamination) were not the result of imposed flexural strain, samples were analyzed in their 'overhang' region - the sample volume outside of the flexural fixture's lower supports, which is not subjected to flexure. $\mu$-CT was performed on a Bruker Skyscan 1173 high energy, spiral scan micro-computed tomography scanner. Image 'slices' were captured with a $70 \mathrm{kV}, 114 \mu \mathrm{A}$ source, using a 1mm aluminum filter, $1300 \mathrm{~ms}$ exposure and a $0.2^{\circ}$ rotary exposure step. With $73 \mathrm{~mm}$ and $364 \mathrm{~mm}$ object-to-source and camera-to-source distances, respectively, slices were capture with $9.95 \mu \mathrm{m}$ resolution. In an effort to characterize worst-case degradation, as indicated by optical microscopy, a $\mu$-CT scan was performed on an unconditioned sample and a threemonth, water exposed samples. Figure 3.14 compares individual, computationally reconstructed slices from the two samples; cross sections illustrated were captured in a plane normal to the direction of specimen length. While voids are present in both samples, qualitatively it was difficult to conclude if there is a difference in void area fraction or distribution.

To quantitatively analyze the obtained image-sets a Python script was developed and implemented. Presented in Appendix E, the script functions by first defining a region-of-interest (ROI), a user-defined region within the selected image. It then measures the greyscale pixel value of each pixel within the ROI-ranging from 0 (black) to 255 (white) - and stores this value in a matrix equal in dimension to the pixel dimensions of the ROI. The script then compares each of the assigned values to a user defined 'threshold' value, below which the pixel is classified as a 'void'. It then iterates through each of the images of the selected dataset, performing the same pixel analysis, and outputs the results to a .csv file for interpretation. To address any 


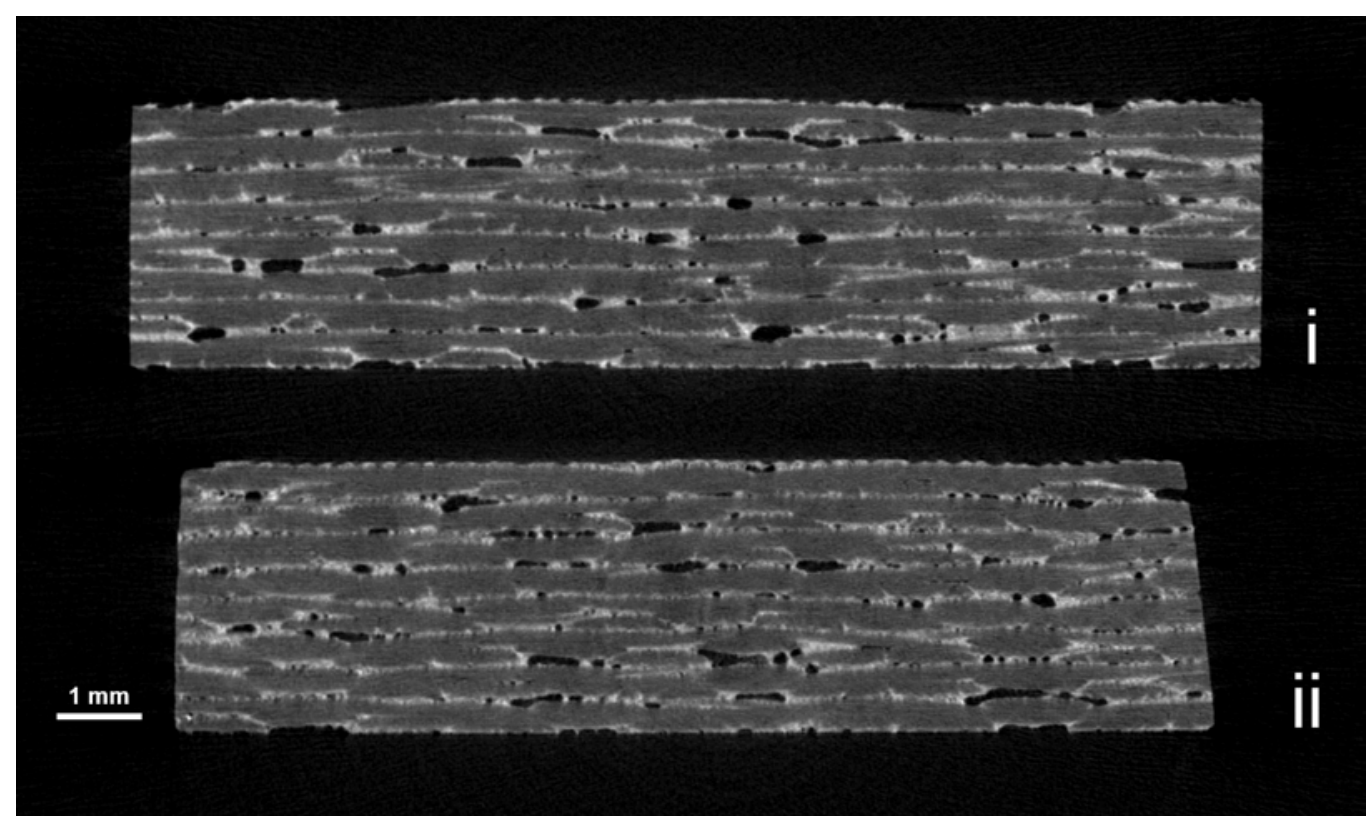

Figure 3.14: Reconstructed cross sectional slices of an i) unconditioned sample and a ii) three-month, DI water exposed sample.

movement of the sample during scanning, the script applies a user defined rotation and translation value to each image, ensuring that the ROI contains only the sample area, omitting the dark region beyond sample boundaries. Figure 3.15 illustrates an exemplary ROI for a single slice and its corresponding void mask, highlighting areas identified as voids. An important note regarding this approach is that, because void detection is restricted to the confines of the ROI and the ROI is defined by the user, detection of voids at the sample's surfaces can be challenging. Either the bounds of the ROI are defined outside of the sample boundaries and regions beyond the sample, including surface voids, are defined as voids, or, the bounds of the ROI are defined inside of the sample boundaries and surface voids are not detected. For a more rigorous evaluation of void formation at sample surfaces, a topographic technique, such as surface profilometry, is required. Alternatively, comparing tomography images of a sample pre- and post-exposure may allow for assessment of void formation at the sample's surface given that images of the degraded sample can be appropriately paired 

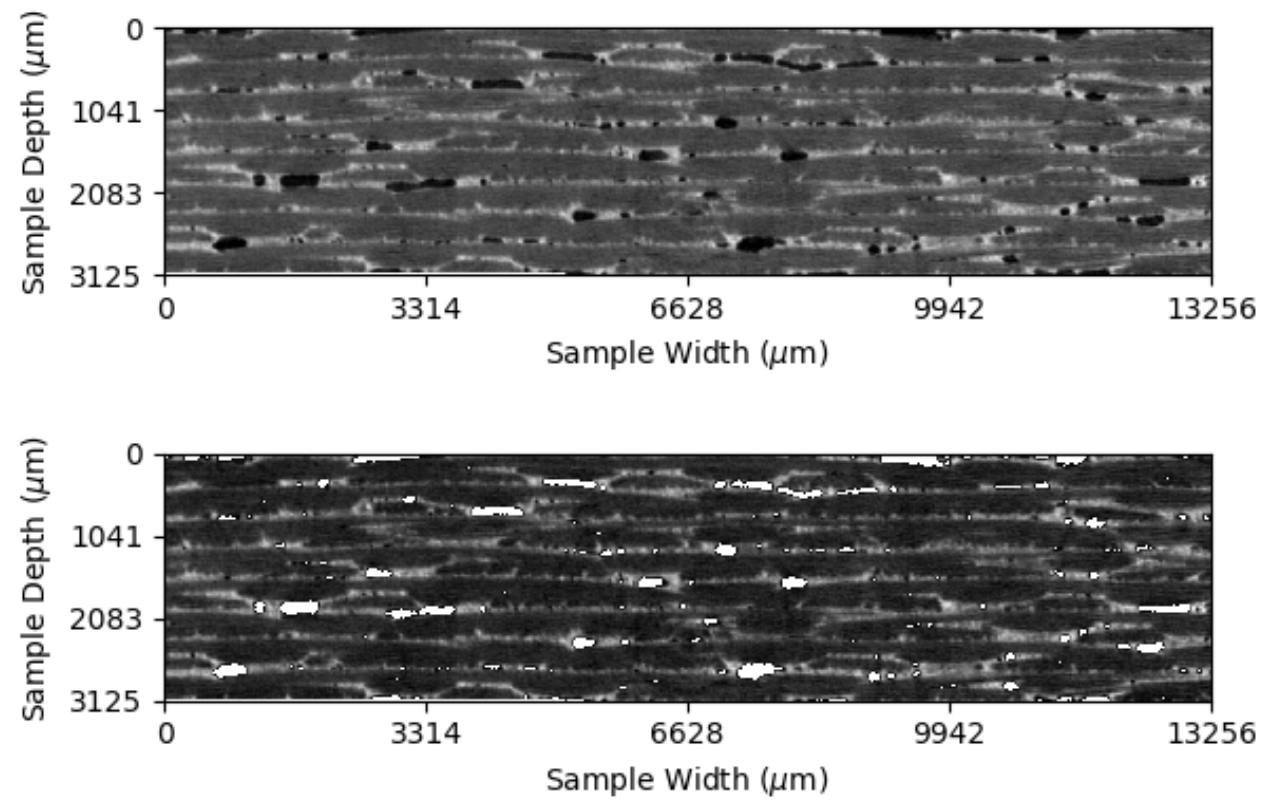

Figure 3.15: Demonstration of void detection; detected voids marked in white.

with their non-degraded counterparts.

From the extracted data, it was determined that the analyzed volumes contained $2.94 \%$ and $2.67 \%$ voids by volume for the unconditioned and three-month, DI water exposed samples, respectively. Hence, the unconditioned sample volume contained $0.27 \%$ more voids by volume than the three-month, DI water exposed sample. While the limited number of samples analyzed prevents determination of the statistical significance of this result, it may be inferred that three months of single-sided exposure to DI water at elevated temperatures has not increased internal void volume, despite clear surface degradation. Figure 3.16 displays the percentage of voids per slice as a function of length along the sample, where 0 represents the start of the analyzed volume with increasing length progressing towards the sample's middle (the point of flexural loading). From this plot two observations can be made. First, void percentage 
shows negligible change globally along the volume's length as indicated by the low slope of the overlaid linear trendlines. This indicates, as anticipated, that stress imposed from flexural testing did not generate voids or delamination in the tested volume nor is there a globally detectable variation in void content from inadequate fabrication practices. Second, there is notable local variation in void content. The unconditioned sample demonstrated that a standard deviation of $0.44 \%$ void content, with maximum and minimum values of $4.15 \%$ and $2.03 \%$, respectively. Assuming voids can occur in the sample volume due only to an absence of matrix material and not fibre, this result suggests that a given cross section can have a matrix content which varies up to $4.61 \%$ by volume. Hence, this result supports that the observed standard deviation in flexural strength is a result of variation in local void content with samples possessing a higher void content directly below the central loading nose exhibiting a lower flexural strength.

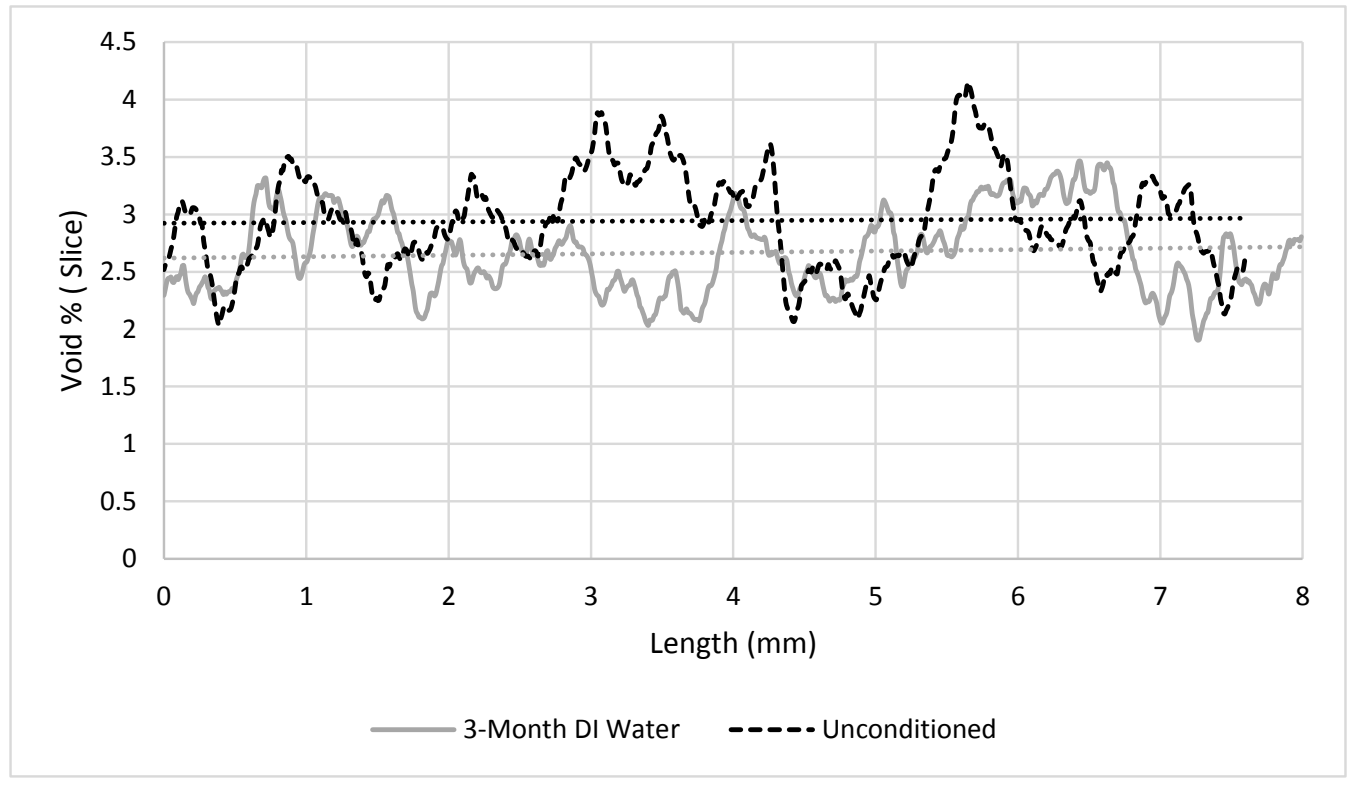

Figure 3.16: Slice void percentage as a function of length of the scanned volume.

Figure 3.17 displays the average void percentage per pixel row as a function of sample depth, where 0 represents the ROI boundary at the bag-surface (the surface exposed 
directly to moisture) with increasing depth progressing towards the tool surface. From this plot, it is observed that there are seven characteristic peaks, each associated with one of the seven interlaminar regions between carbon-fibre fabric plies. Both samples appear to demonstrate a decrease in voids moving toward the sample's center, with higher void concentration at the interlaminar regions closest to the laminate's surfaces. This distribution may be the result of a temperature or pressure gradient present during curing. However, further test replication and statistical analysis are required to establish that this trend occurs throughout the parent laminate. $\mu$-CT has proven to be an effective technique for void evaluation and may prove useful in quality control applications for small components or with traveller coupons of larger components.

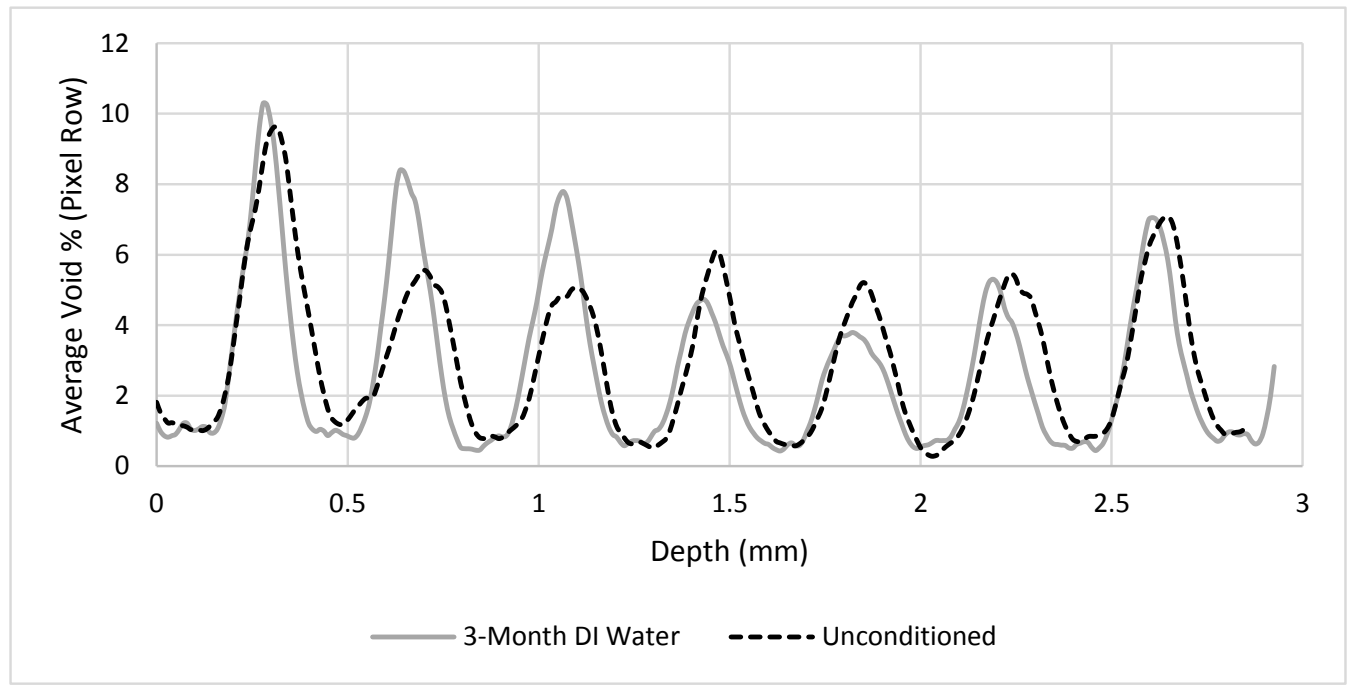

Figure 3.17: Average pixel row void percentage as a function of depth of the scanned volume. 


\subsection{Campaign Two: Immersed SBS}

\subsubsection{Moisture Absorption}

Similar to the first campaign, SBS samples were dried in accordance with ASTM D5229, Procedure D [95], applying identical test parameters to those listed in Table 3.1. Following this, samples were conditioned in accordance with ASTM D5229, Procedure $B$, this time employing full sample immersion. An important deviation from the standard method to note was the used sample mass. ASTM D5229 specifies specimens shall be $\geq 5 g$, established such that an accuracy criterion of $0.02 \%$ change in mass is detectable and to keep the balance approximately ten times more accurate than the required resolution [95]. Tested SBS samples possessed an average preconditioning sample mass of $1.3740 \mathrm{~g}$ hence, a $0.02 \%$ (approximately $3 \mathrm{mg}$ ) change was detectable given a balance accuracy of $\pm 1 \mathrm{mg}$. However, detectable percentage mass change was only three times greater than the required resolution. As a result, SBS sample mass measurements displayed poorer resolution of percentage mass change than flexural samples.

Figure 3.18 presents SBS moisture absorption curves, indicating change in sample mass as a function of conditioning duration, expressed in $\sqrt{\text { hours }}$, for the five chemicals and control investigated. Unlike for the flexural sample moisture absorption study, SBS samples had both a designated sample group which was subjected to continuous moisture absorption readings (hereafter referred to as 'continuous' samples), measured every three to four days, allowing for determination of moisture saturation and, sample groups which had moisture measurements taken once prior to SBS testing (hereafter referred to as 'periodic' samples). Curves plotted as a continuous line represent percentage mass change of continuous samples throughout a nine-week 
duration while curves plotted as a dashed line (group 2) represent percentage mass change measurements of distinct sample groups at their designated time of removal from conditioning, prior to SBS testing. Each data point represents the average percentage change in mass of three samples for a given duration, aside from the continuously monitored DI water samples after $20.8 \sqrt{\text { hours }}$ (18 days) of exposure, this due to a unique phenomena observed, discussed in detail in Section 3.2.1, requiring the sample be removed from conditioning for observation. Continuous sample mass change curves have been smoothed using a three-point moving average to remove variability due to poor mass resolution and any inconsistencies in surface moisture removal processes. An equivalent, non-smoothed curve is presented in Figure D.4 in Appendix D. Measurements taken at $14.69 \sqrt{\text { hours }}$ (nine days) and $16.97 \sqrt{\text { hours }}$ (12 days) have been omitted due to measurement error of the analyst, evident through results which were notably inconsistent with globally observed trends. 


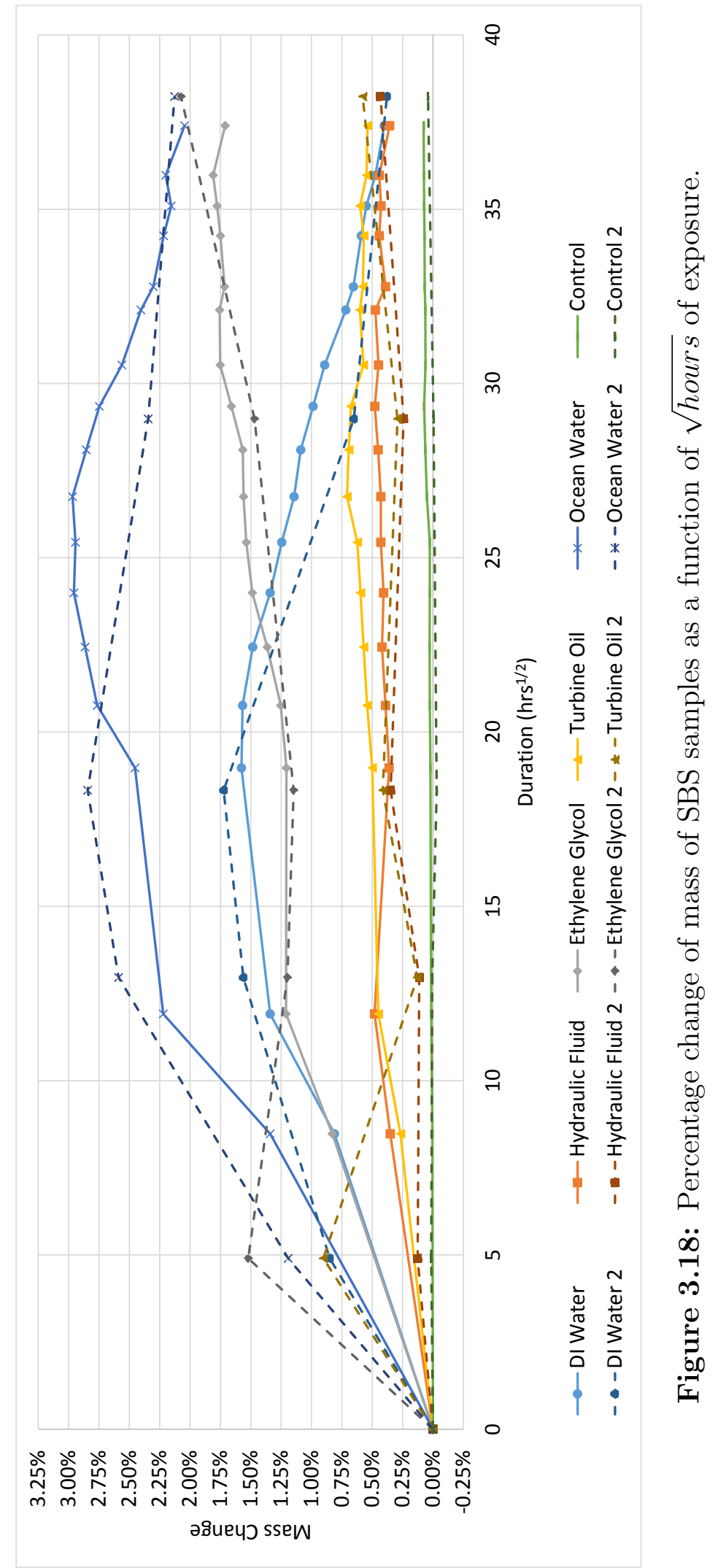


Percentage mass change curves display a number of interesting characteristic of the tested carbon fibre-reinforced epoxy, some of which were not perceivable from the moisture absorption measurements of the flexural samples. Good conformation is evident between the periodic and the continuous samples. With the exception of periodic samples conditioned for $4.90 \sqrt{\text { hours }}$ (1 day) which demonstrated higher mass change values - likely due to inadequate, early-stage surface moisture removal - general moisture absorption trends for periodic and continuous samples are comparable. This suggests that the act of performing continuous, short-term measurements had minimal effect on the obtained data. Turbine oil and hydraulic fluid demonstrated mass change up to approximately $0.5 \%$ at which point a plateau indicative of saturation was reached. Saturation for SBS samples is unverifiable through methods described ASTM D5229, Procedure $B$ due to fluctuations greater than $0.02 \%$ between mass measurements brought about by variability of moisture removal processes and poor percentage mass resolution. Similar to flexural results, control samples show minimal change in mass with a slight long-term increase, likely the consequence of redeposition and ingress of volatilized water or ethylene glycol which has penetrated the imperfect seal of the rubber stoppers.

Ethylene glycol, DI water and ocean water samples all demonstrate trends which are not predicted by a Fickian diffusion model. Ethylene glycol displayed a twostage moisture absorption with a period or rapid absorption followed by a period of slower ingress. Conversely, DI and ocean water samples demonstrated periods of mass increase followed by a crest and a subsequent decrease in sample mass. These two trends are consistent with Weitsman's generalized type $A$ and type $D$ mass-uptake behaviours, respectively, where type $A$ behaviour demonstrates a lack of weight-gain equilibrium and type $D$ behaviour demonstrates mass loss following linear sorption [6]. Weistman suggests $A$ sorption is typically associated with "benign" effects which are 
reversible following desorption while type $D$ are indicative of irreversible fluid-induced degradation.

Figure 3.19 displays the mass change of continuous samples through conditioning and subsequent 12 days of drying at $85^{\circ} \mathrm{C}$. Moisture equilibrium was demonstrated through consecutive daily weighings exhibiting less than a $0.02 \%$ mass change. Dried samples have been offset in duration to best illustrate percentage mass change at an equivalent conditioning duration. As can be observed, ethylene glycol, hydraulic fluid and turbine oil samples returned to baseline mass, with residual changes in mass of $0.28 \%, 0.07 \%$ and $0.16 \%$, respectively. However, DI and ocean water samples demonstrated a $4.75 \%$ and $3.70 \%$ drop in mass from initial equilibrium values. As discussed in regards to flexural samples, this observed mass loss is expected to be a result of irreversible matrix degradation and subsequent leaching of freed material. Differences in mass change between DI and ocean samples can be attributed to residual salt precipitate in ocean water samples. Assuming only the epoxy matrix is degraded from exposure to water and exposed samples possessed a representative fibre mass fraction (60\% fibre, $40 \%$ matrix), a $4.75 \%$ drop in sample mass equates to an approximate $11.9 \%$ loss of epoxy. Based on compositional weight percentage of identified matrix components [1], it can be concluded that the leachant cannot be solely fumed silica, cyanoguanidine or the proprietary epoxy curing agent as each composes less than $3 \%$ of the total composite by weight.

Detection of mass loss of the composite material indicates that mass change cannot be used to predict a fluid absorption model for a system which deteriorates and leaches constituent material into the surrounding environment. While fluid ingress into the matrix may be Fickian-diffusion driven until saturation at sub-macro scalesevaluation of sample mass takes into consideration mass gain and losses of both the diffusant and diffused materials, thus incorporating competing mechanisms of ingress 


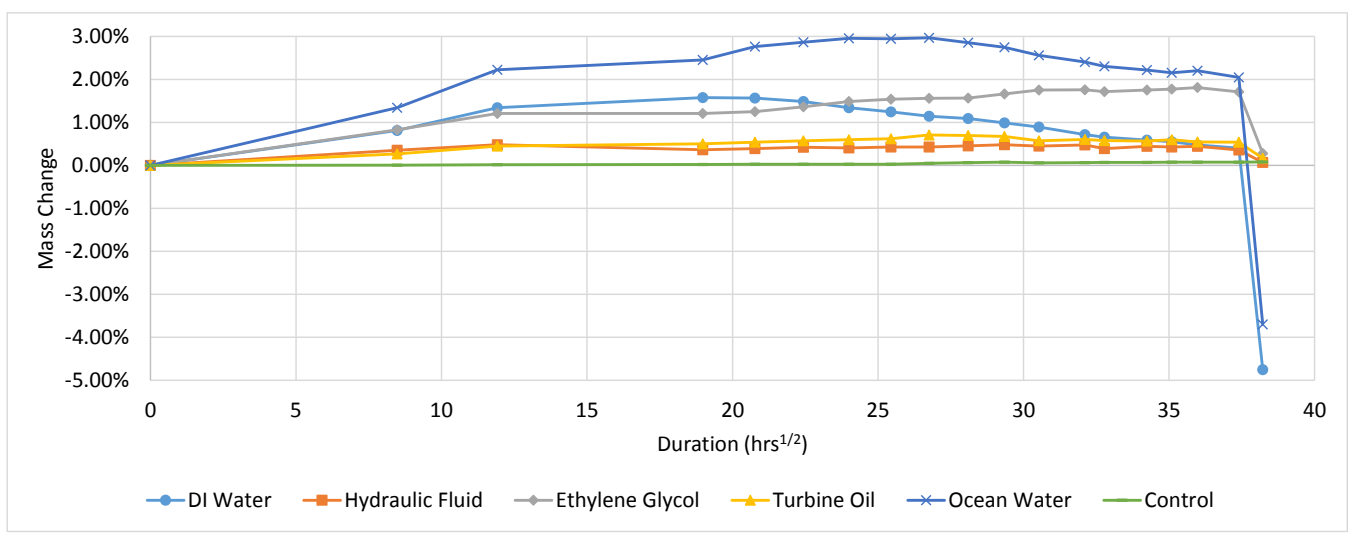

Figure 3.19: Mass change as a function of conditioning duration for continuous sample; dried samples represented by the last data point.

and decay. Hence, accurate evaluation of moisture diffusion in a system of this nature requires evaluation of both ingress and egress of the diffusant and diffused material although, this type of experiment would be logistically difficult to implement.

\section{Leachant Anomaly}

When measuring continuous sample masses following $20.8 \sqrt{\text { hours }}$ (18 days) of exposure, it was noted that one of the DI water sample test tubes had run dry, likely the consequence of vapour pressure forcing the stopper out of the test tube allowing water vapour to escape and liquid to vapourize. Upon measuring the sample's dimensions, it was revealed that the sample had undergone a significant, near $2 \%$ increase in length. Figure 3.20 displays the surface of the dried sample in question which was positioned at the bottom of the test tube. Observable is a translucent and somewhat tacky deposit, seen uniquely on this particular sample. Based on its appearance, but without supportive chemical analysis, it is believed that the deposit has formed from leached epoxy resin. Due to the hemispherical base of the test tube, as water evaporated leached materials was forced to concentrate in a decreasing volume of water. Given a sufficiently high concentration, resin precipitated out on to the surface 


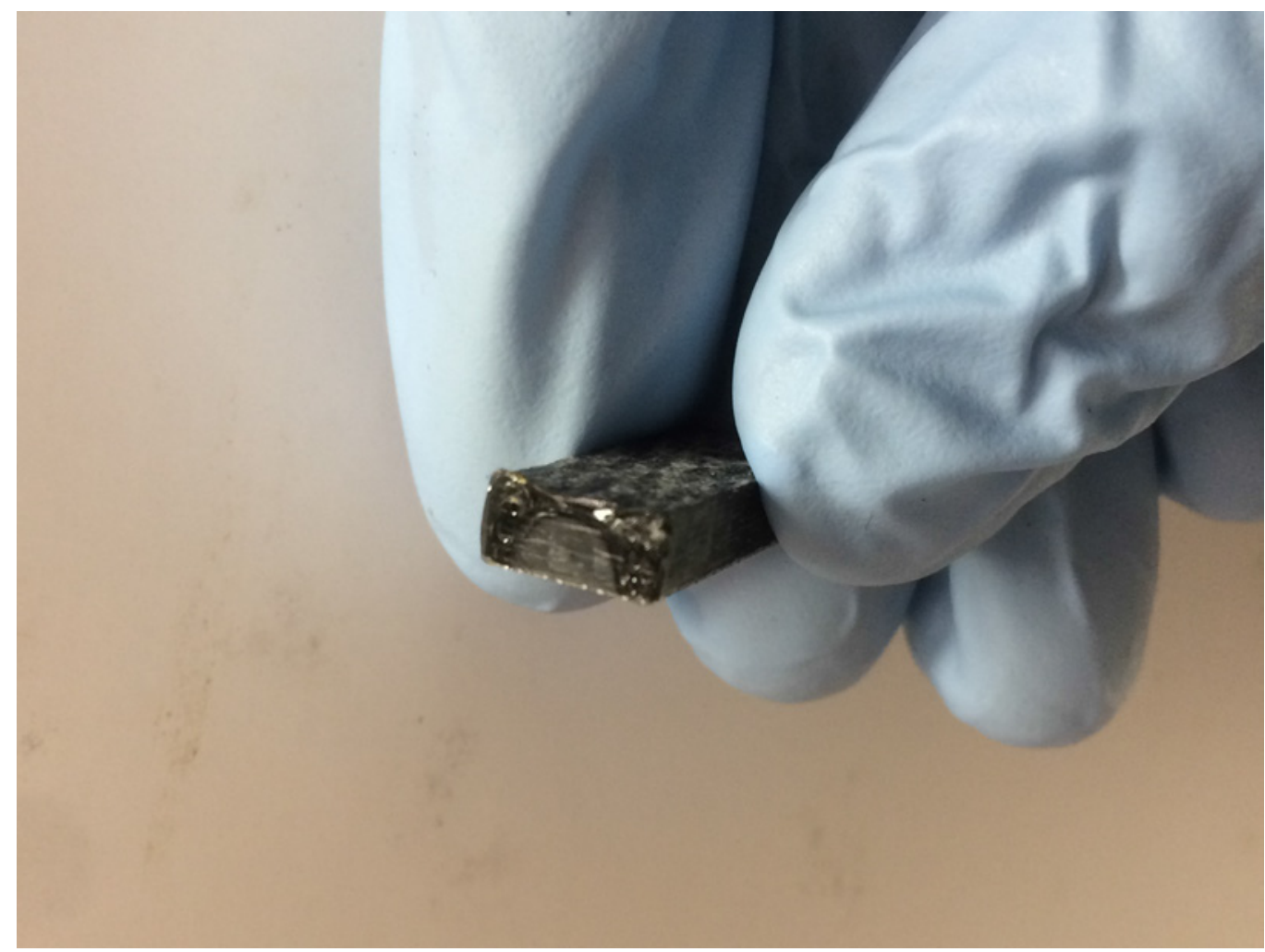

Figure 3.20: Deposit found on SBS sample following sample evaporation.

of the SBS sample. This chance observation further supported matrix leaching as a result of exposure to water and substantiates the white precipitate observed during flexural testing as a component of the matrix. Again, as the matrix is composed of a collection of proprietary chemical ingredients, it is unclear whether the leachant is a single characteristic component or an agglomeration. Further chemical analysis is required to determine specifics of the leachant's composition.

\section{Fickian Modeling}

As only hydraulic fluid and turbine oil samples demonstrated moisture saturation, they are the only fluids which may have their ingress rate accurately predicted using a Fickian model. Recalling Shirrell's criteria for classifying Fickian diffusion [102], 
it can be seen that absorption curves demonstrated concavity to the $\mathrm{x}$-axis prior to saturation however, due lack of early duration sample measurements, it is difficult to confirm linearity up $\geq 60 \%$ of the saturation moisture content. Additionally, as only one sample thickness was tested during each campaign through differing modes of exposure, it is unknown whether samples of different thicknesses will possess superimposable sorption curves. However, as an academic exercise and to illustrate model nonconformity, all samples will be fit to a Fickian diffusion model. This approach will hybridize the rates of linear absorption determined through flexural testing and the saturation mass uptake percentage from SBS testing in order to generate a conservative estimation of Fickian diffusion curves given a single-sided exposure scenario. To do this, a number of approximations and assumptions have been made:

- Hydraulic fluid and turbine oil 'saturation' has been defined as an average of all but the first two measured percentage mass change values

- DI water and ethylene glycol sample saturation has been defined by the maximum observed percentage mass change

- Net moisture absorption ceases once a material reaches saturation

- Material mass loss does not initiate prior to saturation

- Void content does not effect saturation moisture concentration

- Saturation moisture concentration is equivalent for single-sided and immersion conditioning

- Saturation moisture concentration is equivalent for flexural and SBS samples stacking sequences

- Changes in volume resulting from moisture-induced swelling are negated 
Table 3.10 displays the rate of linear absorption, approximated from single-sided exposure data; saturation moisture content, determined from SBS sample immersion and fluid specific diffusion coefficient, $D$, calculated through application of Equation 2.14. Knowing the diffusion coefficient, the moisture absorption function, $G$, can be calculated for all times, $t$, using Equation 2.12. Give an initial moisture content, $M_{i}$, of $0 \%$, percentage change in mass due to moisture ingress can be plotted as a function of exposure duration utilizing Equation 2.13. Figure 3.21 displays resulting Fickian approximations; note that ocean water was not plotted as its variation from DI water absorption profiles is believed to be the result of salt precipitation rather than due to salt driven variations in moisture uptake and saturation.

Table 3.10: Parameters used to approximate Fickian diffusion profiles.

\begin{tabular}{ccccc}
\hline & DI Water & Hydraulic Fluid & Ethylene Glycol & Turbine Oil \\
\hline$\frac{\Delta M}{\Delta \sqrt{t}}\left(\frac{\%}{\sqrt{h r s}}\right)$ & 0.0352 & 0.0280 & 0.0408 & 0.0206 \\
$M_{\infty}(\%)$ & 1.715 & 0.414 & 1.921 & 0.580 \\
$D\left(\frac{i n^{2}}{h r s}\right)$ & $5.253 \times 10^{-6}$ & $5.704 \times 10^{-5}$ & $5.625 \times 10^{-6}$ & $1.573 \times 10^{-5}$ \\
\hline
\end{tabular}

From obtained Fickian curves, using the criteria for determining saturation specified by ASTM D5229 $(<0.02 \%$ change in sample mass over two, seven day reference periods [95]) the exposure duration required to achieve saturation through single sided exposure can be determined. Table 3.11 displays predicted moisture saturation times of each fluid based on approximated Fickian diffusion. From this it can be seen that saturation from single-sided exposure to DI water and ethylene glycol is anticipated to occur in a exposure period $70 \%$ longer than that which flexural samples were subjected to during the first test campaign. However, both hydraulic fluid and turbine oil are predicted to saturate within two-months of continuous exposure. This results suggests that differences in flexural samples from SBS samples, such as greater void content or adsorption to the fractionally greater bag-side surface area, may have 


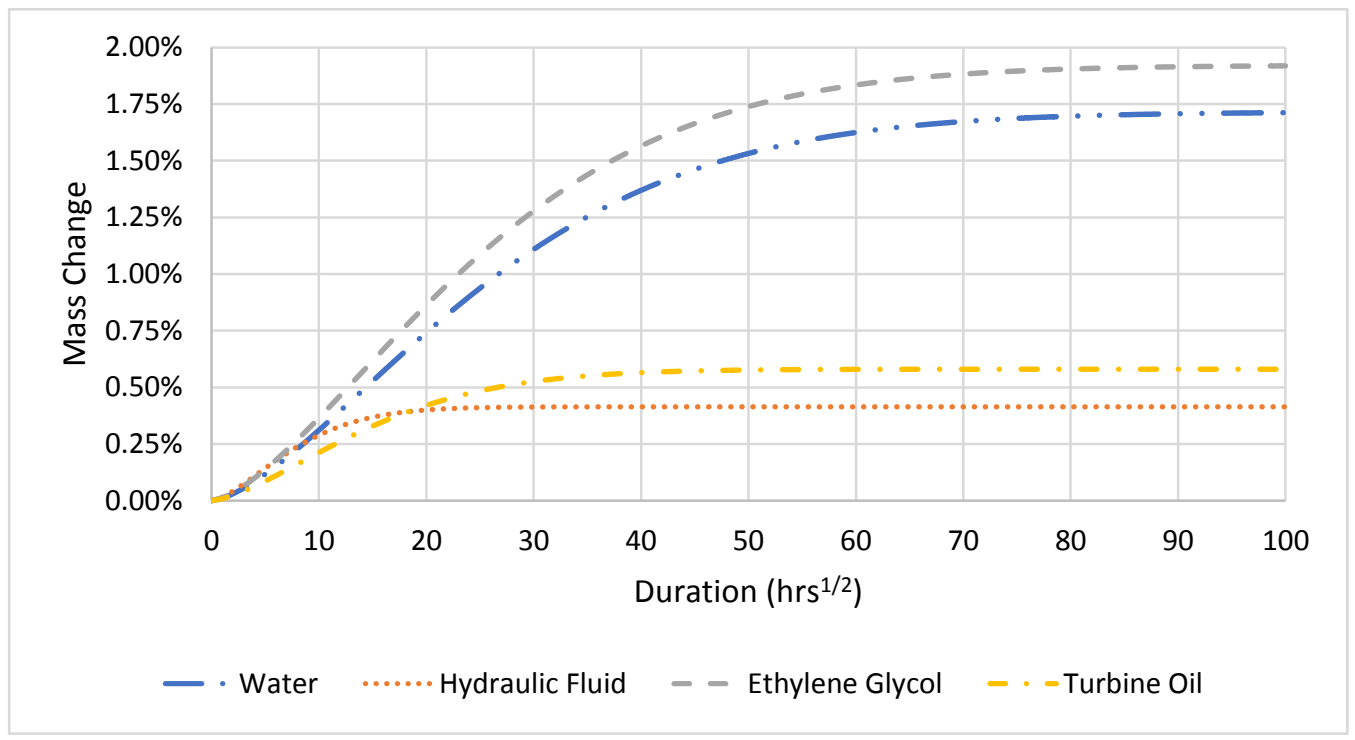

Figure 3.21: Approximated Fickian absorption curves.

Table 3.11: Saturation times by conditioning fluid.

\begin{tabular}{ccccc}
\hline & DI Water & Hydraulic Fluid & Ethylene Glycol & Turbine Oil \\
\hline $\begin{array}{c}\text { Saturation Time } \\
\sqrt{h r s} \text { (Days) }\end{array}$ & $60.6(152.8)$ & $25.7(27.5)$ & $60.7(153.6)$ & $36.8(56.4)$ \\
\hline
\end{tabular}

allowed flexural samples to achieve a greater moisture saturation content. However, determination of ingress rate based on composite structure is beyond the scope of this work.

\section{Temperature Dependence}

As discussed in Section 1.3.2, it is important that results of accelerated ageing experiments can be correlated with in-service conditions. For a maritime aircraft interior, this includes cyclic exposure to a range of temperatures and periodic contamination from various fluids, removed through cleaning or evaporation. To accurately 
predict degradation rates in service, temperature and moisture profiles must be estimated from service logs documenting mission-specific temperatures and contaminations events. However, as these are unavailable for the composite structure under investigation, a conservative estimation of moisture ingression will be performed based on a scenario assuming continuous temperature and moisture conditions.

As this work has not evaluated the effects of temperature on the rates of moisture absorption, a temperature dependent diffusion coefficient, $D(T)$, must be estimated using equation 2.16. In order to determine $D(T)$, the permeability index, $D_{0}$, and activation energy for diffusion, $E_{d}$, must be determined. As these values are traditionally determined empirically for a given fluid-solid system and no work has looked specifically at AX-5180 prepreg, both will be estimated for a water-carbon fibre-reinforced epoxy system based on literature values. As the exact composition of AX-5180 is unknown, diffusion coefficients will be estimated based on a reinforced epoxy consisting of a DGEBA resin cured with an amine-based hardener.

In their work studying the effects of moisture on carbon fiber-reinforced epoxy composites, Augl and Berger performed an extensive DOE study looking at the diffusion coefficients in six carbon composites, made from a combination of three different resins and two different fibres [111]. One of the resins investigated was DER 332, a DGEBA epoxy which was cured with 4,4' diaminodiphenyl sulfone hardener. Under identical humidity and temperature conditions, diffusion coefficients for DER 332 were comparable for both fibre types tested. As such, approximations will be based on data obtained for DER 332 in combination with Thornel 300, 3K carbon fibre. Composite panels were formed from 8 plies of unidirectional prepreg with an approximate $70 \%$ fibre volume fraction. Diffusion coefficients were determined using a humidity chamber at two relative humidities, $30 \%$ and $80 \%$. Immersion of a neat Normco 5208 epoxy 
resin demonstrated similar diffusion coefficients for $80 \%$ and $100 \%$ relative humidity at $30^{\circ} \mathrm{C}$, hence values will be approximated from composite diffusion coefficient determined at $80 \%$ relative humidity.

Figure 3.22 displays the natural logarithm of diffusion coefficients as a function of inverse temperature. By plotting diffusivity and temperature in this manner, the activation energy of diffusion and permeability index can be determined from the plot's slope and y-axis intercept. Hence, $E_{d}$ and $D_{0}$ were determined to be 46.55 $\mathrm{kJ} / \mathrm{mol}$ and $2.11 \times 10^{3} \mathrm{~cm}^{2} / \mathrm{s}$, respectively. These results show good confirmation to literature values for different epoxy resins presented in the same work [111] and to those presented elsewhere, looking at the diffusion coefficient of a DGEBA epoxy coating [112]. Using these values, $D(T)$ can be calculated for any temperature $T$. Percentage mass change can then be plotted as function of exposure duration through determination of $G$ for every time, $t$.

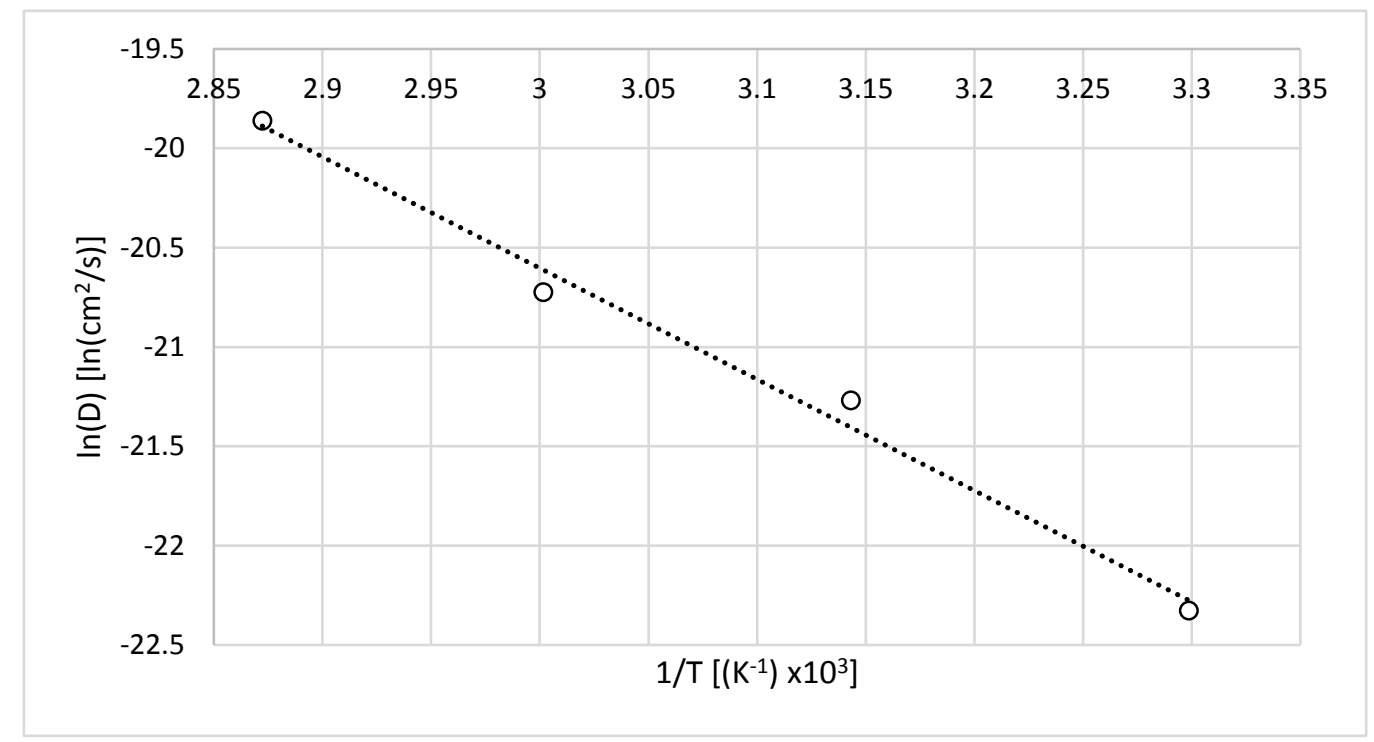

Figure 3.22: Natural logarithm of diffusivity as a function of inverse temperature.

Figure 3.23 displays percentage mass gain as a function of exposure duration for a range of conditioning temperatures, were $100 \%$ signifies moisture saturation. While it 
is important to recall discrepancies between the observed mass uptake and a Fickian model as well as required assumptions, Figure 3.23 provides a clear contrast between moisture absorption at $85^{\circ} \mathrm{C}$ and that which can occur at $20^{\circ} \mathrm{C}$. Again, using the criteria for determining saturation specified by ASTM D5229, conditioning at $85^{\circ} \mathrm{C}$, saturation is reached in 48 weeks, while conditioning at $20^{\circ} \mathrm{C}$, saturation is predicted after 12.6 years of continuous exposure. Of course, this is not a realistic exposure scenario for typical in-service conditions of a maritime aircraft interior for which the cyclic nature of exposure must be considered. Temperature dependent absorption profiles for other fluids investigated can not be estimated due to a lack of literature studying the association between alternative fluid ingress and conditioning temperature.

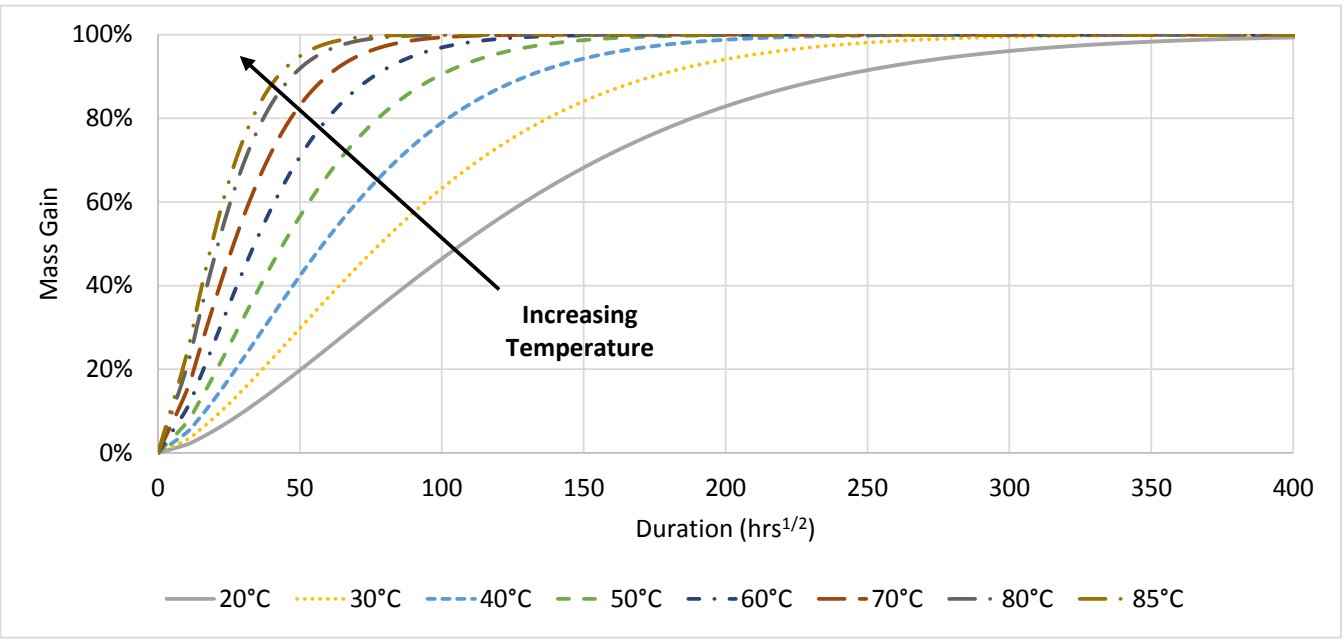

Figure 3.23: Percentage mass gain as a function of exposure duration; $100 \%$ represents saturation.

\subsubsection{Sample Metrology}

Dimensional analysis was performed following sample mass measurements. Each sample was measured three times at different locations along their length, width and 
thickness with average dimensional standard deviations of $0.1 \%, 0.3 \%$ and $0.6 \%$, respectively. Table 3.12 presents the two-factor ANOVA performed on metrological data obtained from SBS samples at their designated conditioning duration. Similar to flexural data, percentage change of sample length did not demonstrate statistical significance as a result of variations in chemical type, exposure duration or their interaction. Sample width demonstrated statistically significant due to chemical type, but not due to exposure duration or their interactions. This suggests that initial chemical exposure, over a single day of conditioning, caused an immediate change in sample width which did not evolve with time. Change in sample thickness demonstrated statistical significance both due to chemical type and exposure duration encouraging further investigation. These results support that directional dependent swelling observed for flexural testing is due to the quasi-isotropic nature of length and width directions in contrast to their anisotropy with the thickness direction, and not due to the conditioning fixture design.

Figures 3.24, 3.25 and 3.26 display average percentage changes in SBS sample length, width, and thickness, respectively, as a function of conditioning duration for continuously monitored samples. Percentage change in dimensions has been displayed over an equivalent range to demonstrate the relative effects of conditioning on each dimension. As predicted by ANOVA, changes to sample length are negligible, with less than $0.4 \%$ change detected for all samples and no discernible long-term trends. Change to sample width is negligible for DI water, hydraulic fluid, turbine oil and control samples, with a consistent, near $1 \%$ increase detected for ocean water and ethylene glycol samples. Change to sample thickness is observably significant for DI and ocean waters samples which exhibit curves possessing similar profiles to those obtained for moisture absorption data; early-stage increase followed by a crest and subsequent decrease. Again, differences in DI and ocean water thickness change may 
Table 3.12: Two-factor ANOVA summary for change in SBS sample length, width and thickness; significant results highlighted in bold.

\begin{tabular}{|c|c|c|c|c|c|}
\hline $\begin{array}{l}\text { Source of } \\
\text { Variation }\end{array}$ & $\begin{array}{l}\text { Sum of } \\
\text { Squares }\end{array}$ & $\begin{array}{c}\text { Degrees of } \\
\text { Freedom }\end{array}$ & $\begin{array}{c}\text { Mean } \\
\text { Square }\end{array}$ & $F_{0}$ & P-value \\
\hline \multicolumn{6}{|c|}{ Length } \\
\hline Chemical & $1.46 \mathrm{E}-04$ & 5 & $2.93 \mathrm{E}-05$ & 4.75173 & 0.001008 \\
\hline Time & $4.60 \mathrm{E}-05$ & 4 & $1.15 \mathrm{E}-05$ & 1.86360 & 0.128600 \\
\hline Interaction & $1.34 \mathrm{E}-04$ & 20 & $6.72 \mathrm{E}-06$ & 1.09042 & 0.382784 \\
\hline Error & $3.70 \mathrm{E}-04$ & 60 & $6.17 \mathrm{E}-06$ & & \\
\hline Total & $6.97 \mathrm{E}-04$ & 89 & & & \\
\hline \multicolumn{6}{|c|}{ Width } \\
\hline Chemical & $3.46 \mathrm{E}-03$ & 5 & $6.92 \mathrm{E}-04$ & 8.51347 & 0.000004 \\
\hline Time & $7.32 \mathrm{E}-04$ & 4 & $1.83 \mathrm{E}-04$ & 2.25107 & 0.074088 \\
\hline Interaction & $3.06 \mathrm{E}-03$ & 20 & $1.53 \mathrm{E}-04$ & 1.88294 & 0.031127 \\
\hline Error & $4.88 \mathrm{E}-03$ & 60 & $8.13 \mathrm{E}-05$ & & \\
\hline Total & $1.21 \mathrm{E}-02$ & 89 & & & \\
\hline \multicolumn{6}{|c|}{ Thickness } \\
\hline Chemical & $3.56 \mathrm{E}-02$ & 5 & $7.13 \mathrm{E}-03$ & 61.91498 & $2.09 \mathrm{E}-22$ \\
\hline Time & $4.28 \mathrm{E}-03$ & 4 & $1.07 \mathrm{E}-03$ & 9.28820 & 0.000007 \\
\hline Interaction & $5.77 \mathrm{E}-03$ & 20 & $2.88 \mathrm{E}-04$ & 2.50444 & 0.003252 \\
\hline Error & $6.91 \mathrm{E}-03$ & 60 & $1.15 \mathrm{E}-04$ & & \\
\hline Total & $5.26 \mathrm{E}-02$ & 89 & & & \\
\hline
\end{tabular}


be attributed to salt precipitation observed throughout the study and perceivable from ocean water's disparity from baseline thickness following drying. Ethylene glycol's demonstrated mass increase throughout conditioning in not reflected in thickness measurements which showed nominal difference from the negligible changes observed for turbine oil, hydraulic fluid and control samples.

Literature has described the volume in a thermosetting polymer as either occupied or free. Occupied describes a volume which is taken up by either molecular mass itself or the space from which it excludes other molecules as a result of thermally dependent harmonic vibrations [113]. Free describes a volume which is unoccupied by molecular mass, due to molecular packing irregularity, forming nanoscopic voids [113]. Furthermore, in epoxy, swelling has been shown to be a result of absorbed water molecules hydrogen bonding with epoxy hydroxyl groups, disrupting inter-chain hydrogen bonding, increasing intersegmental hydrogen bond length, thus the increasing occupied volume [113]. Hence, observed mass increase without corresponding volume increase suggests that absorbed moisture occupies only the composite's free volume and does not disrupt intermolecular bonds. This is an important observation as it supports that resulting changes in sample volume due to moisture absorption are indicative of a decrease in mechanical properties of the epoxy matrix, a relationship which is reflected in both flexural properties and SBS data, discussed in Section 3.2.3. 


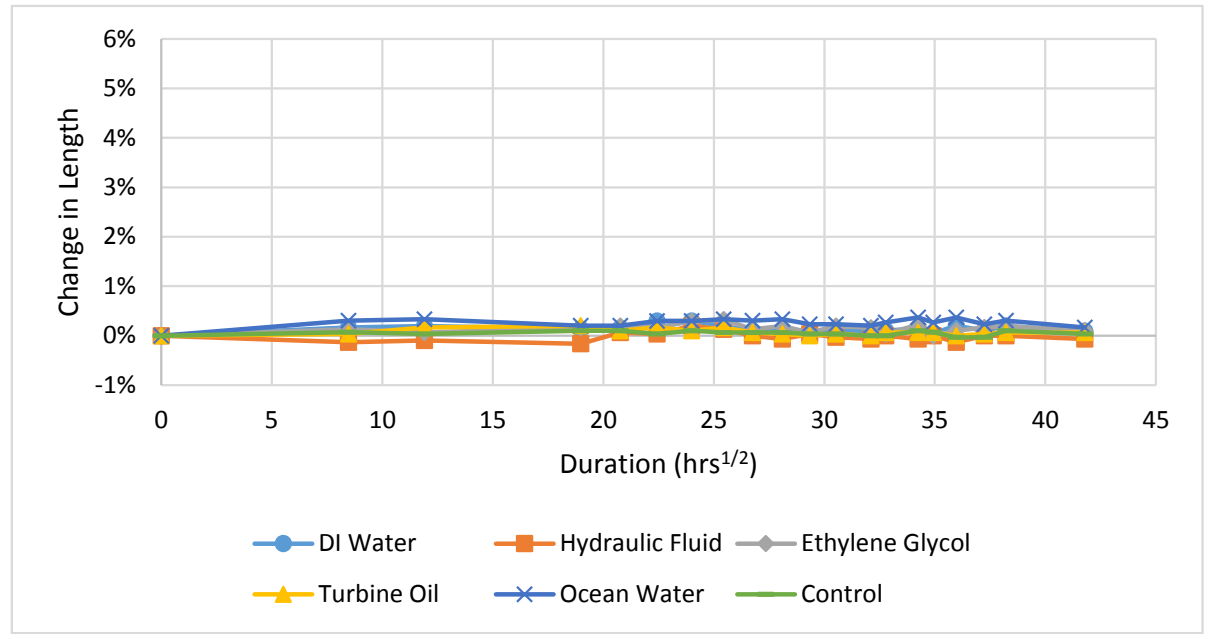

Figure 3.24: Percentage change in SBS sample length as a function of conditioning duration; last point represents dimension following drying.

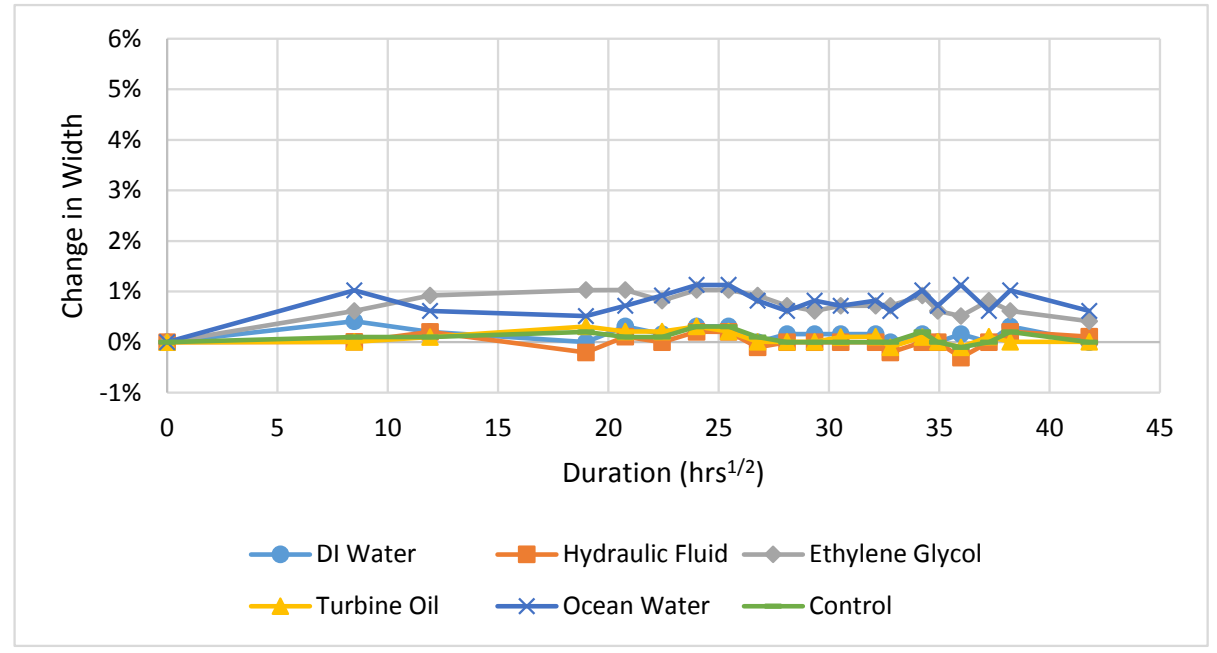

Figure 3.25: Percentage change in SBS sample width as a function of conditioning duration; last point represents dimension following drying. 


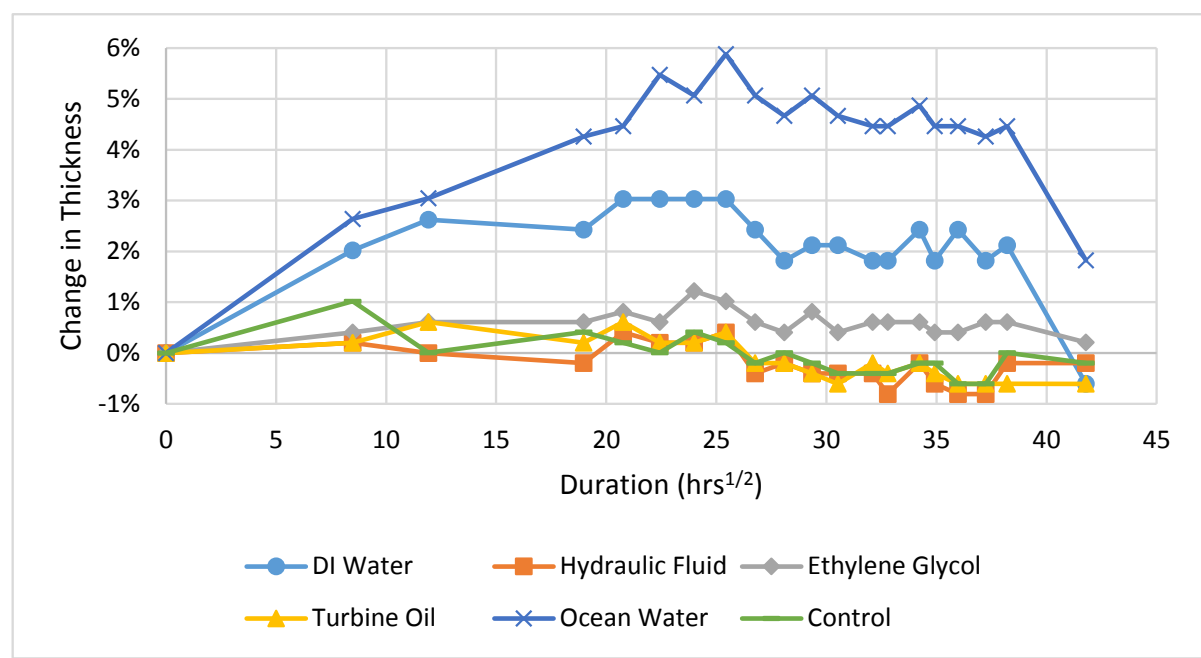

Figure 3.26: Percentage change in SBS sample thickness as a function of conditioning duration; last point represents dimension following drying.

Figure 3.27 presents the linear coefficient of moisture expansion as a function of conditioning duration for fluid conditioned samples. Here, a positive value indicates thickness has increased with mass uptake and a negative value indicates that thickness has decreased with mass uptake. A consistent value with increasing duration indicates a change in thickness proportional to mass uptake. What can be observed is that DI water, ocean water and ethylene glycol exposed samples demonstrated a positive CME, while turbine oil and hydraulic fluid demonstrate both positive and negative CMEs, inconsistently. As mass change data demonstrated saturation of hydraulic and turbine oil samples is achieved after as early as $12 \sqrt{\text { hours }}$ (six days), variability of CME may be attributed to variability in thickness measurements, likely do to inaccuracy of the caliper used and inconsistencies in the bag-side sample surface. As both fluids induced a relatively small change in mass, variability in thickness measurements are amplified in the CME. Thus, changes to the CME of turbine oil and hydraulic fluid are inconclusive. Ethylene glycol exhibits a consistent CME around 0.5, demonstrating a proportional one-dimensional increase per unit of mass absorbed. DI and ocean water samples initially demonstrated a consistent CME just under 2.0 
however, after $30 \sqrt{\text { hours }}$ (36 days) DI water samples exhibited an increase in the CME. This rise is associated with the leaching of material from the samples. As the matrix material is more dense than water (approximately $1.35 \mathrm{~g} / \mathrm{cm}^{3}$ ), mass is lost while sample volume is maintained by water occupying the freed volume. Interestingly, ocean water samples exhibited a consistent CME throughout this period. Assuming ocean water provoked an equivalent rate of fluid uptake and matrix decay to that of DI water, this suggests that salt precipitation equilibrated mass loss to mass gained.

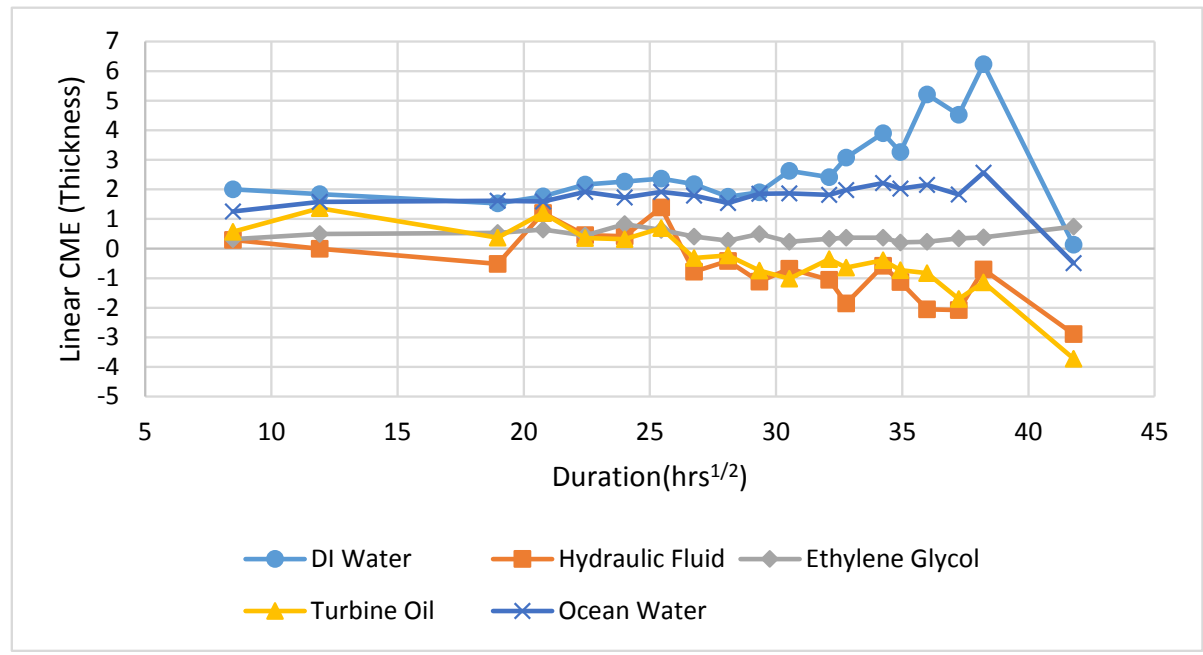

Figure 3.27: CME as a function of exposure duration; last point represents CME following drying.

\subsubsection{Short Beam Strength}

SBS testing was performed in accordance with ASTM D2344 [88]. Table 3.13 outlines the specifications of ASTM D2344 and any required deviations. Use of $5 \mathrm{~mm}$ support rollers was necessitated by the flexural fixture's incompatibility with small roller sizes. When using large rollers relative to sample thickness, non-negligible changes in span length occur due to a shift in the point of tangency during flexure [90]. This is an 
important consideration when comparing SBS of samples with varying thickness but is not of concern for relative comparison of samples of an equivalent geometry for which absolute strength values are not of interest. Width and thickness measurements were taken using a caliper, due to equipment availability, and three replicates were tested to accommodate available oven volume.

Table 3.13: Specified and utilized test parameters for ASTM D2344; deviations from standard are highlighted in bold.

\begin{tabular}{|l|l|l|}
\hline \multicolumn{1}{|c|}{ Test Parameter } & \multicolumn{1}{c|}{ Specification } & \multicolumn{1}{c|}{ As Tested } \\
\hline Loading nose and supports shape & smooth, cylindrical & smooth, cylindrical \\
\hline Loading nose diameter & $6.0 \pm 0.50 \mathrm{~mm}$ diameter & $6.0 \mathrm{~mm}$ diameter \\
\hline Support diameter & $3.0 \pm 0.40 \mathrm{~mm}$ diameter & $\mathbf{5 . 0} \mathbf{~ m m ~ d i a m e t e r ~}$ \\
\hline Loading nose and supports hardness & $60-62 \mathrm{HRC}$ & $\sim 62$ HRC \\
\hline Width and thickness metrology & $\begin{array}{l}\text { Micrometer, } 4-5 \mathrm{~mm} \text { ball-interface, } \\
\text { suitable for reading within } 1 \%\end{array}$ & $\begin{array}{l}\text { Caliper, suitable } \\
\text { for reading within } \mathbf{1 \%}\end{array}$ \\
\hline Length metrology & $\begin{array}{l}\text { Micrometer or caliper, suitable } \\
\text { for reading within } 1 \%\end{array}$ & $\begin{array}{l}\text { Caliper, suitable } \\
\text { for reading within } 1 \%\end{array}$ \\
\hline Number of replicates & five & three \\
\hline Fibre composition & $\geq 10 \% 0^{\circ}$ span direction, & $45.5 \%$ span direction, \\
& balanced and symmetric & balanced and symmetric \\
\hline Length-to-thickness ratio & 6 to 1 & 6 to 1 \\
\hline Width-to-thickness ratio & 2 to 1 & 2 to 1 \\
\hline Crosshead speed & 1 mm/min & 1 mm/min \\
\hline
\end{tabular}

Figure 3.28 presents the percentage change in SBS strength as a function of conditioning duration. Samples which were conditioned for nine weeks and then dried are represented by individual points, free from the primary curve. Presented results show a clear degradation of SBS as a result of exposure to both DI water and ocean water with, after nine weeks of conditioning, a measured $52.5 \%$ and $54.4 \%$ decrease, respectively. Similar to flexural results, two-factor ANOVA of SBS (the summary of which has been presented in Table D.2 of Appendix D), demonstrates statistic correlation 


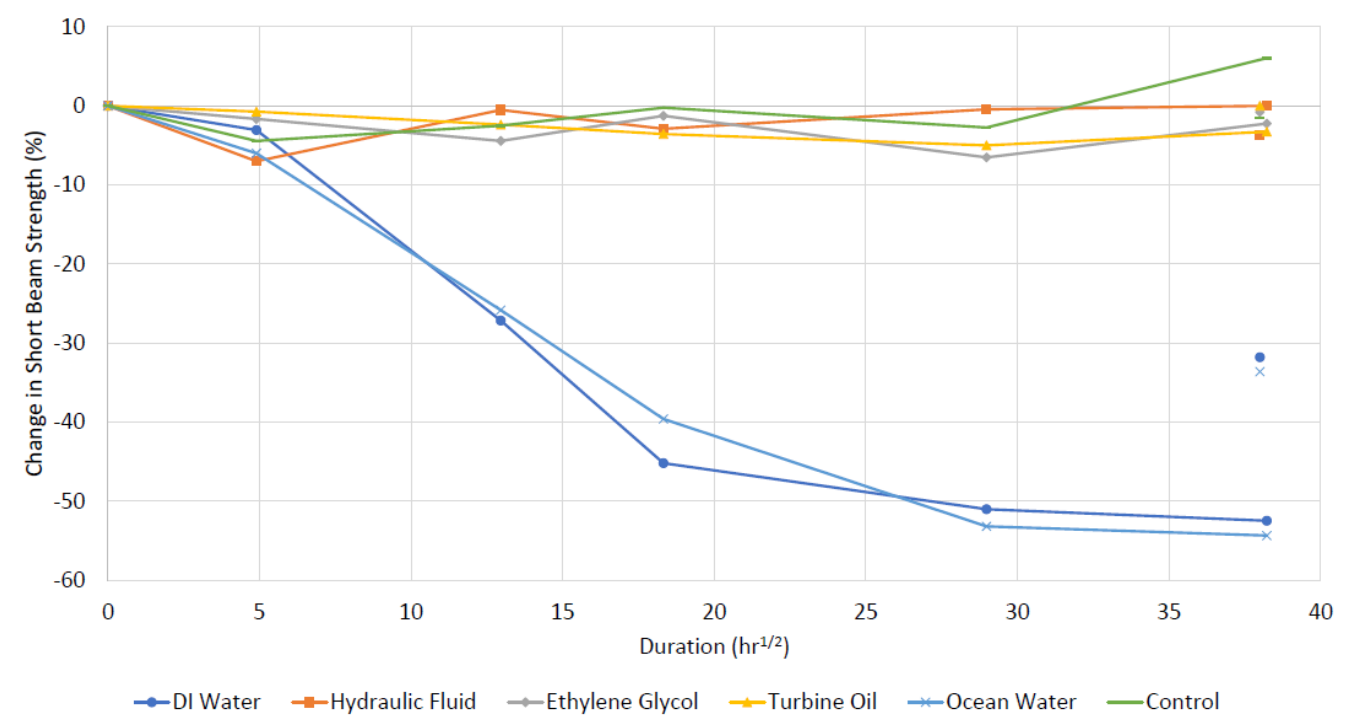

Figure 3.28: Change in short beam strength as a function of exposure duration; dried samples represent by individual data points free from the curve.

between the contributions of chemical exposure, exposure duration and their interaction on change in SBS using a $p=0.01$ cutoff. Dunnett and Tukey post hoc analysis, summarized in Table D.3 of Appendix D, shows a statistically significant effect of DI and ocean water conditioning at exposure durations greater-than or equal to one week, but did not show statistically significant difference in degradation from each other. Conversely, hydraulic fluid, turbine oil, ethylene glycol and control samples did not demonstrate a statistically significant differences from average, baseline SBS for any of the tested exposure durations. While most sample groups showed a decrease in SBS - with the exception of nine-week control, hydraulic fluid and dried, turbine oil samples - degradation was maintained at less than $10 \%$ with no observable, definitive trends. This result supports those interpreted for flexural, hydraulic fluid, turbine oil, DI water and ocean water samples, but refutes trends observed for control and ethylene glycol samples. Hence, validation of long-term post cure of flexural control samples and degradation of flexural ethylene glycol samples requires supplementary evaluation. 
Following drying, nine-week DI water and ocean water samples showed a $43.5 \%$ and 45.4\% increase, respectively, in the SBS from their, moisture saturated counterparts. Dried samples retained an approximate $33 \%$ decrease in SBS from baseline values. Indication of both reversible and irreversible changes to mechanical properties supports interpretation of chemical effects suggesting observed cumulative degradation due to both disruption of intersegmental hydrogen bonding and intramolecular chain scission. While the time dependence of intermolecular and intramolecular degradation mechanisms cannot be deduced from this work, their contributions to SBS strength can be approximated assuming their fractional contributions to total degradation are time-independent. Figure 3.29 displays approximated inter-, intramolecular and total induced degradation from water ingress. This approximation assumes that intermolecular interactions are completely reversible, a likely unrealistic assumption due to steric interactions, however one which requires further investigation. Interestingly, plotting water induced degradation of SBS as a function of $\sqrt{\text { hours }}$ of exposure resembles the inverse plot of Fickian diffusion; linear mass uptake (degradation) to $\geq 60 \%$ of saturation (ultimate degradation) with concavity to the $\mathrm{x}$-axis until saturation. Additional experimentation is required to determine if a plateau in SBS is achieved over longer duration exposures, or if a continuous decrease is detected. This observation suggests that degradation induced by exposure to water can be predicted by a Fickian diffusion model. However, as demonstrated through analysis of percentage mass change data, prediction of moisture absorption in a diffusant which exhibits decay and egress requires a more sophisticated evaluation technique.

A direct comparison of percentage change of flexural and SBS strengths has been presented in Figure D.5 of Appendix D. Results are inconsequential due to differences in stacking sequence, exposure route and duration. Prominent features include the approximate linear decrease of flexural strength with anomalous one-month data, 
contrasted with linear decrease of SBS followed a plateau in strength. Longer conditioning durations are required to verify whether changes to flexural strength due to single-sided exposure exhibit a similar trend to immersed SBS samples.

It is difficult to provide a direct comparison between the obtained mechanical results and those presented in literature, due to variations in conditioning fluid, testing duration, composite composition, composite layup, conditioning temperature and static property evaluated. However, generalized trends observed for DI and ocean water test cases are comparable to those previously achieved, that is, an observable, statistically significant decrease in matrix-dominant mechanical properties.

Limited studies on the effects of hydraulic fluid contamination demonstrates mixed results. Work by Sugita et al. demonstrated comparable rates of a Skydrol 500B hydraulic fluid ingression and negligible effects of hydraulic fluid on the hardness of carbon/epoxy monolithic specimens [64]. Conversely, their works demonstrated a significant drop in the hardness of a hydraulic fluid-exposed epoxy-based adhesive. Landry et al. demonstrated a $4 \%$ decrease to the delamination toughness of carbon/epoxy composites immersed in Aeroshell Fluid 41 hydraulic fluid, but showed up to a $66 \%$ increase in delamination growth rate for hydraulic fluid exposed samples [66]. Skydrol 500B is primarily composed of dibutylphenylphosphate [114], while Aeroshell Fluid 41 is composed of a nondescript mixture of hydrodesulfurized gas oils [115]. Literature supports that the exposure of carbon/epoxy composites to hydrocarbon-based hydraulic fluid does not cause significant decrement to static mechanical properties. However, hydraulic fluid may pose a more significant risk to epoxy-based adhesives or fatigue, necessitating further research.

Both studies also examined the effects of anti-icing fluids - Sugita tested a diethylene glycol monomethyl ether-based Prist Hi-Flash anti-icing fuel additive and Landry 
tested the effects of a ethylene glycol-based UCA ADF XL 54 deicing fluid. The former showed significant degradation to anti-icing fuel additive-exposed adhesive specimens, with complete degradation of samples at elevated temperatures (70 and $85^{\circ} \mathrm{C}$ ) and a $13 \%$ drop in hardness of carbon/epoxy samples while the later showed a $22 \%$ decrease in delamination toughness of deicing fluid-exposed samples and a $327 \%$ increase in delamination growth rate. Mixed results between the static properties observed in the current study and literature necessitates further testing, studying the effects of various chemical-composite combinations to identify specific detrimental chemical interactions. No literature was found evaluating the effects of turbine oil contamination on the mechanical properties of composites, hence obtained result can not be compared.

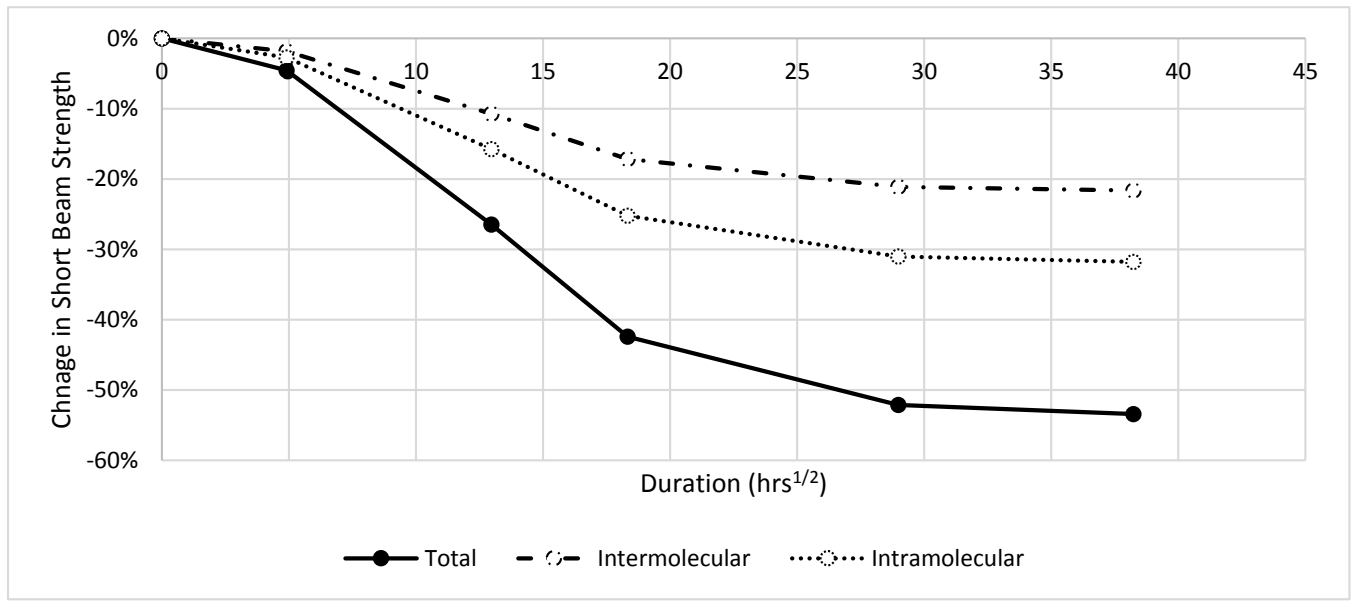

Figure 3.29: Hypothesized inter- and intramolecular contributions to change in short beam strength as a function of exposure duration assuming timeindependent contributions.

\subsubsection{Hardness Evaluation}

Hardness testing was carried out in accordance with ASTM D2240 [116] which details the evaluation of durometer hardness - an indentation hardness technique intended 
primarily for elastomers but also applicable for thermoplastics and thermosets. ASTM D2240 describes hardness measurements for 12 durometer scales, each characterized by a unique indentor type indented for a specific range of hardness values. In general, harder materials are tested with smaller radii indentors such that a lower force can induce a greater penetration. Table 3.14 outlines the specifications of ASTM D2240 and any required deviations implemented.

Table 3.14: Specified and utilized test parameters for ASTM D2240; deviations from standard highlighted in bold.

\begin{tabular}{|c|c|c|}
\hline Test Parameter & Specification & As Tested \\
\hline Indentor shape & Test specific & Type D \\
\hline Indentor material & $\begin{array}{l}\text { Steel rod, hardened to } \\
500 \text { HV10 }\end{array}$ & $\begin{array}{l}\text { Steel rod, hardened } \\
\text { to } 500 \mathrm{HV} 10\end{array}$ \\
\hline Presser foot & $\begin{array}{l}\text { Flat, circular, } \\
18 \pm 0.5 \mathrm{~mm}\end{array}$ & $\begin{array}{l}\text { Flat, circular, } \\
18 \pm 0.5 \mathrm{~mm}\end{array}$ \\
\hline Indicator display & $\begin{array}{l}\text { Digital or electronic, } \\
100 \text { equal divisions }\end{array}$ & $\begin{array}{l}\text { Digital, } 100 \\
\text { equal divisions }\end{array}$ \\
\hline Indentor displacement & $\begin{array}{l}0.025 \mathrm{~mm} \text { per } \\
\text { durometer unit (Type D) }\end{array}$ & $\begin{array}{l}0.025 \mathrm{~mm} \text { per } \\
\text { durometer unit }\end{array}$ \\
\hline Spring calibration & $\begin{array}{l}0.4445 \mathrm{~N} \text { per indicator } \\
\text { division (Type D) }\end{array}$ & $\begin{array}{l}0.4445 \mathrm{~N} \text { per } \\
\text { indicator division }\end{array}$ \\
\hline Operating stand & $\begin{array}{l}\text { Applies presser foot } \\
\text { parallel to support table }\end{array}$ & No stand \\
\hline Specimen support table & Solid flat surface & Granite slab \\
\hline Specimen thickness & $\geq 6.0 \mathrm{~mm} \dagger$ & $4.14 \mathrm{~mm}$ \\
\hline Specimen lateral dimensions & $\begin{array}{l}\text { Large enough to allow } \\
\text { measurements }>12.0 \mathrm{~mm} \\
\text { from edge } \dagger\end{array}$ & $25.12 \times 8.26 \mathrm{~mm}$ \\
\hline
\end{tabular}

$\dagger$ dimensions may be less than specified if results demonstrate independence from proximity to sample boundaries 
Hardness testing was performed in an effort to evaluate its capacity to capture degradation due to chemical ingress in an in-service scenario. Hence, measurements were taken manually, without a stand to produce an accuracy achievable in the field. Specimen dimensions were restricted by the specifications of ASTM D5229, however, baseline hardness testing of the parent laminate showed there to be no variation in hardness values resulting from sample edge effects.

Hardness testing was performed using a Shimana analog durometer. Following conditioning and mechanical testing, SBS samples were subjected to three hardness measurements taken at the points indicated in Figure 3.30. Two measurements, points one and three, were taken peripheral to the point of contact of the loading nose with the intention of evaluating change in hardness due to fluid exposure while point two, taken at the point of contact of the loading nose, intended to show change in hardness resulting from mechanical loading and induced yield. Baseline, unconditioned samples possessed an average Shore D hardness of 92.4 at peripheral measurements and 86.0 at the loading nose. A similar difference in hardness was noted between the loaded and peripheral measurements for all sample groups although, as each sample did not receive the same maximum loading, quantitative evaluation could not be performed. Variability in hardness readings was believed to be a result of variations in topography of the sample bag-side surface, local voids concentration beneath the indentor, and off-axis (non-perpendicular) application of the durometer, a consequence of manual application.

Figure 3.31 presents percentage change is sample hardness as a function of exposure duration taken as an average of six peripheral measurements per sample group. Samples which were conditioned for nine weeks and then dried are represented by individual points, free from the primary curve. What can be interpreted is that DI and ocean water samples exhibited a notable drop in hardness, after as early as one 


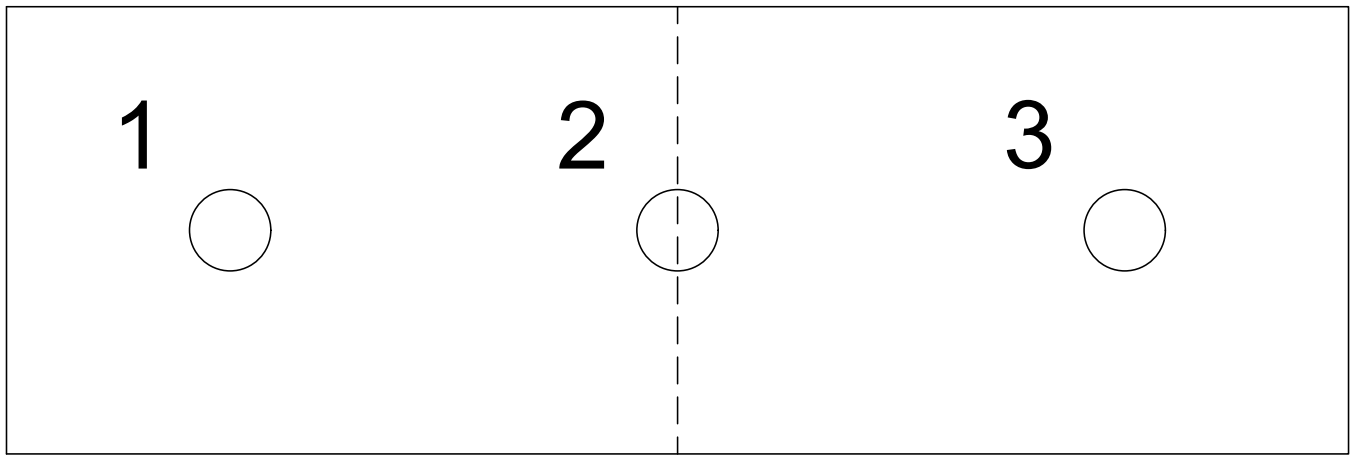

Figure 3.30: Schematic of SBS sample bag-side surface with hardness measurements numbered; dashed line indicates point of contact of loading nose.

day of exposure, which remained fairly constant throughout testing. Hydraulic fluid, turbine oil and control samples showed a smaller drop in hardness and ethylene glycol samples demonstrated a hardness which progressively decreased. Applying Dunnett's test, assuming all sample groups exhibited a normal hardness distribution, using a two-tailed approach, with a $99 \%$ confidence interval, it was demonstrated that ocean water exhibits a statistically significant change in hardness after as early as one day of conditioning and both DI and ocean water samples showed consistently significant results after two weeks of conditioning. Ethylene glycol samples exposed for nine weeks were the only other sample group to demonstrate significance.

Following drying, DI water, ocean water and ethylene glycol samples demonstrated an increase in hardness with a final value insignificantly different from baseline hardness values. This result indicate that changes in hardness readings are a result of a reversible plasticization of the epoxy matrix induced by the ingress of water and ethylene glycol molecules. As flexural and SBS data demonstrated, ethylene glycol induced less significant intermolecular interaction with the epoxy matrix. This suggests why changes to ethylene glycol sample hardness demonstrated significance only over long-duration exposure. It may be theorized that, because water exposed samples demonstrated an early-reached plateau which does not reflect observed sample 


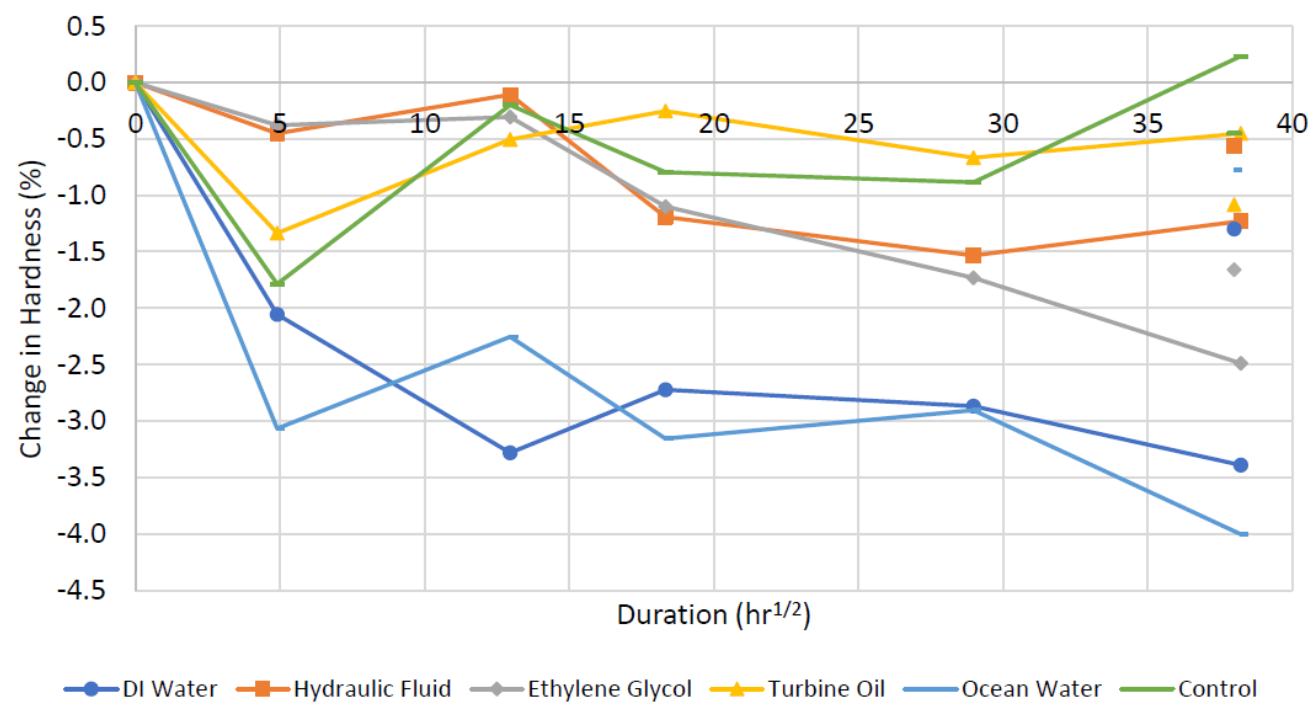

Figure 3.31: Change in hardness as a function of exposure duration; dried samples represent by individual data points free from the curve.

mass uptake trends, hardness measurements were only capable of capturing moisture absorption in the surface epoxy layer. As indentation hardness is evaluated from the indentor's ability to permanently deform a material under compressive loading, if it encounters an undeformable material, that material will exhibit maximum hardness. Carbon fibre demonstrates a hardness greater than epoxy, one which can not be characterized by a Shore D durometer scale. Hence, while the indentor is capable of deforming surface epoxy, sub-surface carbon fibre does not deform under its applied load. Thus, measurable difference in indentor penetration characterized only a difference in the penetrable epoxy which saturates more rapidly than that of internal layers.

From the interpreted results it can be concluded that Shore D durometer hardness testing is capable of indicating the presence of ingressed moisture, but is incapable of quantifying the amount of moisture contained in a carbon-fibre reinforced epoxy laminate. Additionally, Shore D durometer hardness testing is capable of detecting material damage resulting from interlaminar shear although it cannot be said if it is 
capable of quantifying imposed damages.

\subsubsection{Thermal Analysis}

In order to prepare samples for thermal analysis, nine-week-conditioned SBS samples, which had previously been subjected to SBS testing and temporarily re-immersed in fluid, were removed from fluid and sectioned into thin slices using a water-cooled diamond blade on a UKAM Smart Cut 6001 GP Sectioning Saw. Sample slices were then re-immersed in fluid for transport prior to thermal analysis. Once ready for testing, samples slices were removed from fluid and further broken up using tweezers. Sample pieces were then weighed and portioned, attempting to maintain a representative fibre-volume fraction, and loaded into their respective sample pans.

\section{Differential Scanning Calorimetry}

DSC was conducted in accordance with ASTM E1356 [117] on a TA Instruments Q20 Differential Scanning Calorimeter. Samples, loaded in a Tzero aluminum sample pan, were heated from $40^{\circ} \mathrm{C}$ to $200^{\circ} \mathrm{C}$ at a rate of $10^{\circ} \mathrm{C}$ per minute, held at isothermal for one minute then cooled to $40^{\circ} \mathrm{C}$ at a rate of $10^{\circ} \mathrm{C}$ per minute while being subjected to a continuous $50 \mathrm{~mL}$ per minute flow of nitrogen purge gas. Throughout the thermal cycle, heat flow was continuously evaluated relative to a reference pan, at a rate of two readings per second. Using the sample mass, evaluated prior to thermal testing, heat flow per gram of sample was determined as a function of temperature.

Figure 3.32 displays a plot of the heat flow per gram of sample as function of temperature in ${ }^{\circ} \mathrm{C}$, comparing a single representative sample conditioned using each fluid type. Endothermic processes are represented by negative values while exothermic 


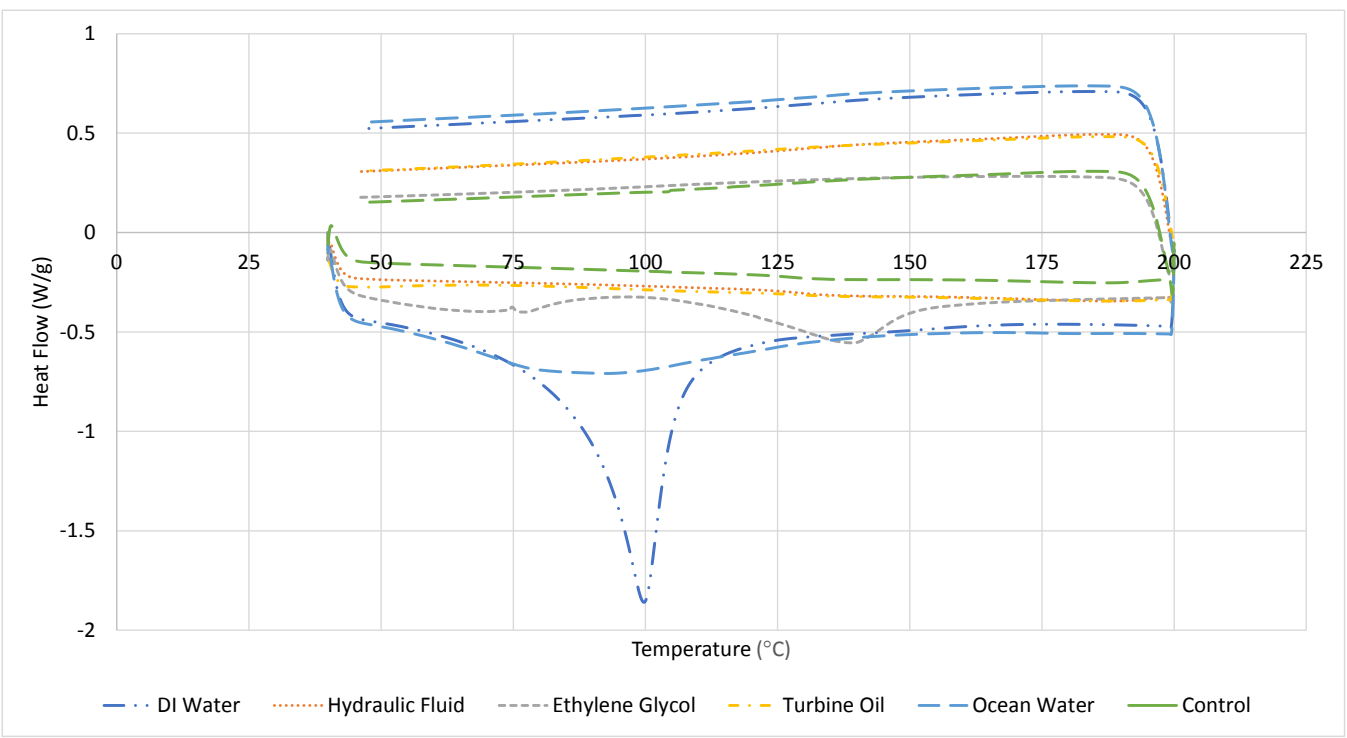

Figure 3.32: Comparison of heat flow per gram as a function of temperature during DSC heating cycle for representative nine-week samples.

processes are represented by positive values. From this plot, visible exothermic peaks in DI water, ocean water and ethylene glycol sample curves can be associated with fluid evaporation, as verifiable through comparison with characteristic TGA fluids evaporation curves presented in Figures D.6 and D.8 of Appendix D.

$\mathrm{T}_{\mathrm{g}}$ is a kinetic transition, represented by a step function in a heat flow vs. temperature curve, associated with a change in a material's heat capacity, due to a change in chain mobility, between rigid and viscous states. Figure 3.32 does not display a discernible $\mathrm{T}_{\mathrm{g}}$ for any of the plotted samples. Figure 3.33 shows only a plot of the control sample's DSC curve, allowing for greater y-axis resolution. From this plot an inflection point is visible during the heating process at approximately $134^{\circ} \mathrm{C}$. Similar isolated observation of hydraulic fluid and turbine oil plots shows a common inflection points in this region during heating $\left(127\right.$ and $129^{\circ} \mathrm{C}$, respectively) but, similarly, do not demonstrate the reverse transition during cooling. Difference in interpreted glass transition from the supplier specified value of $151^{\circ} \mathrm{C}$ [35] is anticipated to be due to differences in evaluation technique; the supplier's technical data sheet specifies use of 
dynamic mechanical analysis for $\mathrm{T}_{\mathrm{g}}$ determination but does not provide the utilized heating rate nor whether the onset of storage modulus, peak of the loss modulus or peak of tan delta curves were used to specify $\mathrm{T}_{\mathrm{g}}$. Analysis of DI water, ocean water and ethylene glycol curves does not present a discernible $\mathrm{T}_{\mathrm{g}}$, likely as the relatively small change is baseline heat flux at $T_{g}$ has been masked by the larger magnitude endothermic evaporation events.

Tests of baseline unexposed material demonstrated similar result to that of the control, hydraulic fluid, and turbine oil samples; an inflection point discernible around $130^{\circ} \mathrm{C}$ during heating with no visible transition during cooling. For comparison, an amorphous polystyrene standard was tested on the same equipment using a similar heating cycle. Results demonstrated that a characteristic step function during both heating and cooling with a transition in baseline heat flow of about $0.25 \mathrm{~W} / \mathrm{g}$. Thus, the relatively small shift in baseline heat flow at $T_{g}$ for the carbon-epoxy material tested is likely a consequence of both the small mass of epoxy within the extracted material (approximately $3.4 \mathrm{mg}$, assuming a 40\% weight fraction) and a high degree of crosslinking in the cured material, limiting chain mobility. Lack of transition during cooling is characteristic of the crystallization of mobile amorphous regions, possible in DGEBA depending upon the inter-epoxy chain length [7]. Further testing is re-

quired to validate this proposition. Nonetheless, due to poor resolution and lack of consistent detection, little may be inferred from DSC results regarding the effects of fluid exposure on the tested fibre-reinforced epoxy laminate.

\section{Thermogravimetric Analysis}

TGA was conducted in accordance with ASTM E1131 [118] on a TA Instruments Q50 Thermogravimetric Analyzers. Samples, loaded in a platinum sample pan, were 


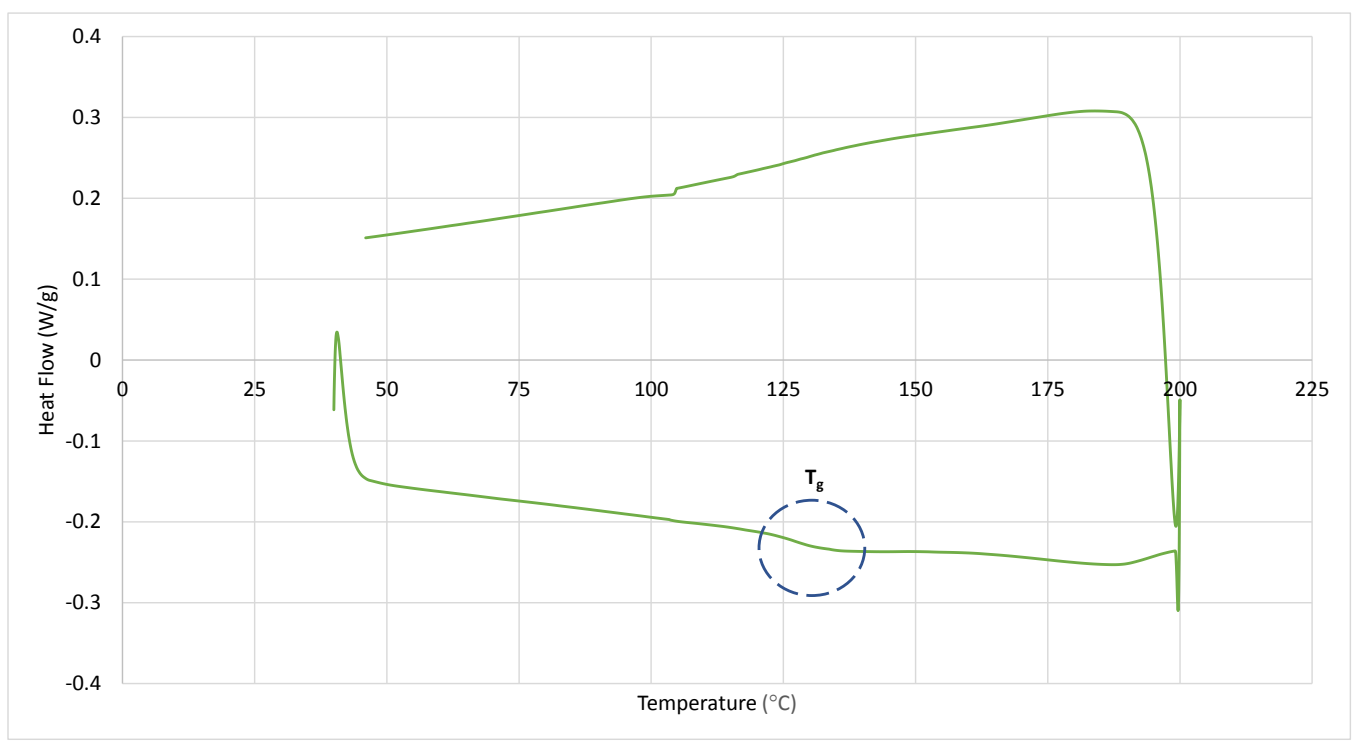

Figure 3.33: Heat flow per gram as a function of temperature during DSC heating cycle for a representative nine-week control sample.

heated from ambient temperature to $900^{\circ} \mathrm{C}$ at a rate $30^{\circ} \mathrm{C}$ per minute while being subjected to a continuous $60 \mathrm{~mL}$ per minute flow of nitrogen purge gas. During heating, sample masses were continuously evaluated at rate of one readings per second allowing for the determination of percentage change in sample mass as a function of temperature. Figure 3.34 displays a plot of the percentage sample weight as a function of temperature, in ${ }^{\circ} \mathrm{C}$, comparing a single nine-week sample conditioned with each fluid type. Loss of sample mass is associated with the release of volatiles formed through either fluid evaporation or material degradation. Drops in sample mass at temperatures lower than $300{ }^{\circ} \mathrm{C}$ are primarily the result of fluid evaporation, as confirmed by comparison with characteristic TGA fluid evaporation curves presented in Figures D.7 through D.9 of Appendix D. In general, evaporation temperatures are lower than those observed for baseline fluid samples, likely a result of the higher surface area of the small volume of fluid present. As samples were immersed following mechanical testing and sectioning, changes in sample mass cannot be quantitatively correlated with ingressed fluid. 


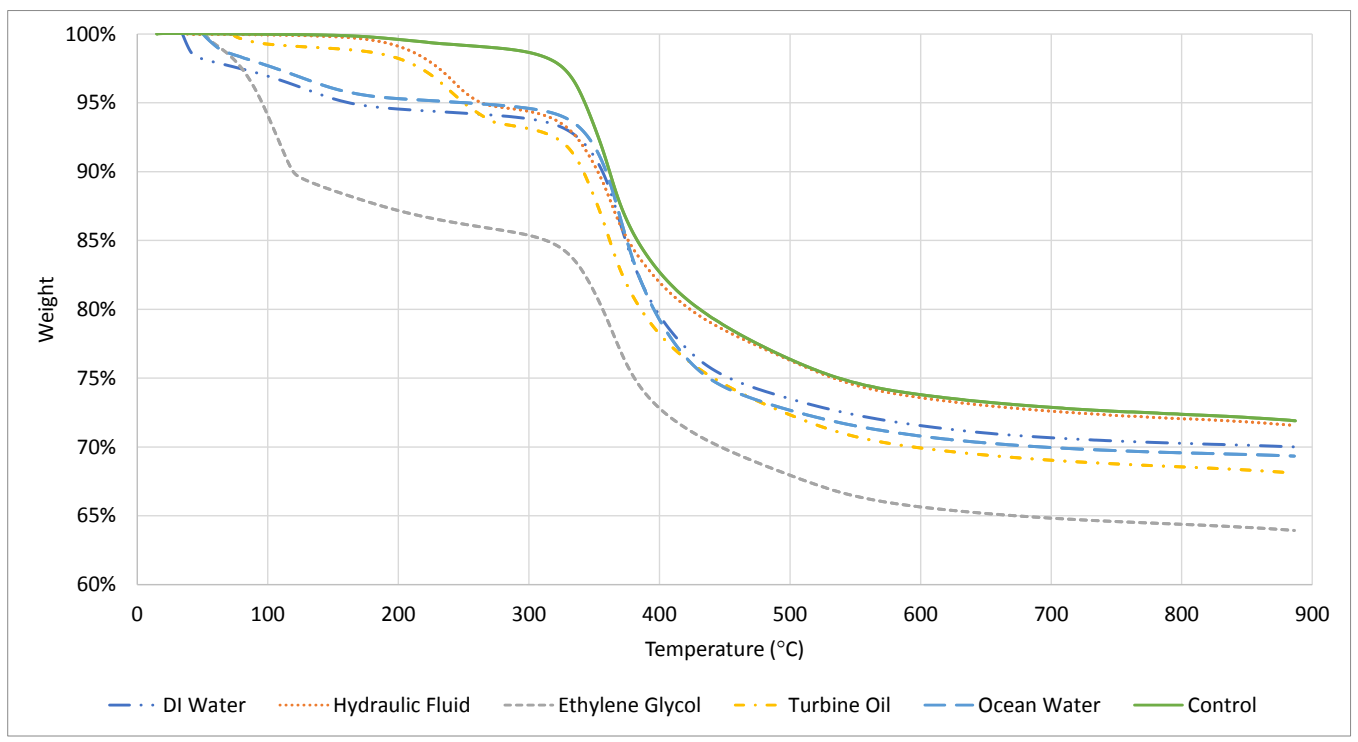

Figure 3.34: TGA plot of percentage residual mass as a function of temperature.

Following fluid evaporation, each sample assumes a curve characteristic of the control sample; prior to $300{ }^{\circ} \mathrm{C}$ a few percent drop in mass is observable, likely the result of either localized heating of the sample or volatilization of trapped residual, uncured resin. Above $300{ }^{\circ} \mathrm{C}$ there is a distinct drop in mass, associated with the degradation of cured epoxy, followed by a plateau signifying the end of volatilization. Figure 3.35 displays percentage change in sample weight, given 100\% represents sample mass following fluid evaporation, prior to epoxy degradation. As can be seen, sample curves can be superimposed with slight deviations in final sample mass, the result of variations in sample fibre volume ratio. DI and ocean water samples demonstrated a slightly higher degradation onset temperature likely due intermolecular interactions of ingressed fluid, although further testing is required to validate this hypothesis. 


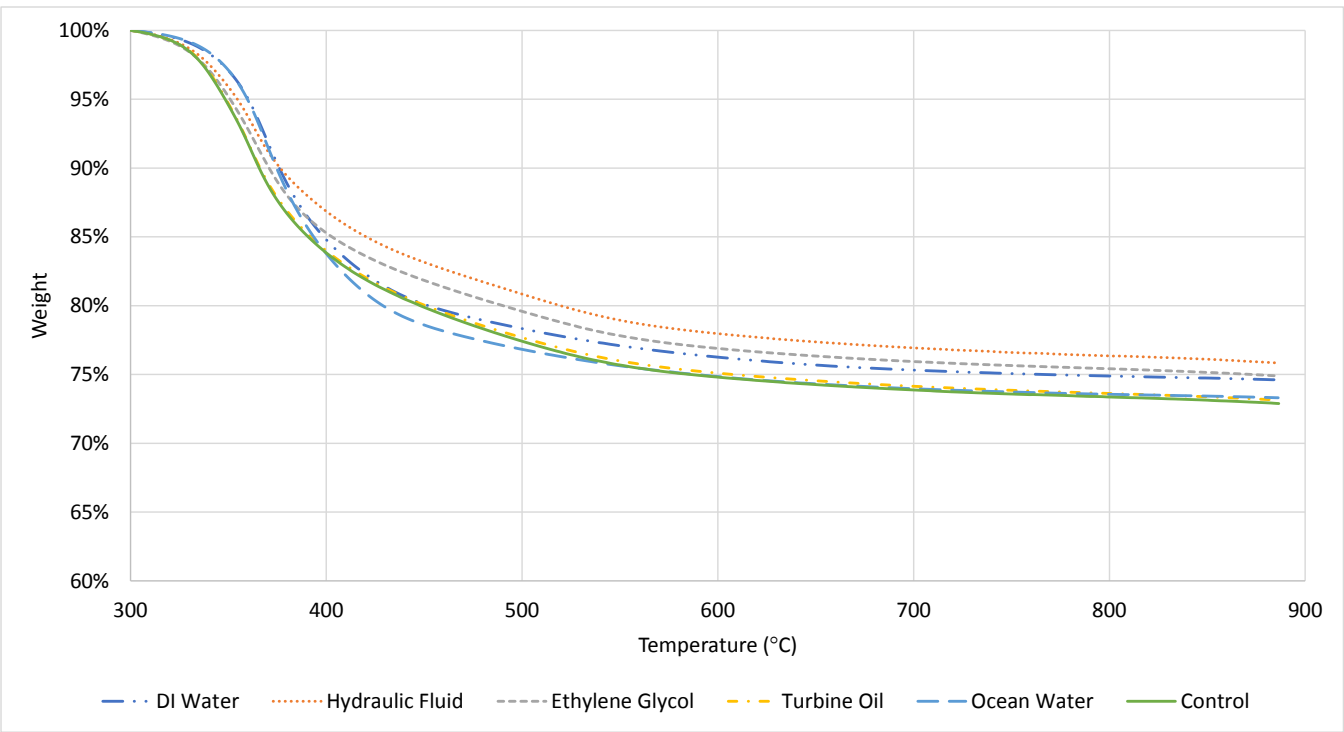

Figure 3.35: TGA plot of percentage residual mass as a function of temperature; initial sample mass represents sample mass following ingressed fluid evaporation. 


\section{Chapter 4}

\section{Experiment Evaluation}

As a novel conditioning technique, there were a number of nuances and obstacles associated with the employed single-sided exposure investigation which, inherent to the method or encountered through testing, required deviation from standard practices. This chapter discusses complications of the methods used, their influence on testing and potential biases on the obtained results. Areas discussed include observed temperature variances, fluid evaporation and gasket moisture uptake.

\subsection{Temperature Variance}

ASTM D5229 standard specifies conditioning temperature shall be maintained at the temperature of interest, $\pm 3^{\circ} \mathrm{C}$ [95]. As discussed in Chapter 2, preliminary analysis of the laboratory oven temperature distributions revealed that, while it was possible to obtain an even, stable $85^{\circ} \mathrm{C}$ in an empty oven, it was not possible to achieve this distribution for all arrays when four arrays were positioned as shown in Figure 2.12. While temperatures were stable in all positions, the large surface area of the arrays

hindered effective heat transfer through convection currents, generating a differential 
between the bottom and top arrays based on proximity to the heating coils. To mitigate temperature variance, conditioning arrays were repositioned weekly to allow an equal exposure duration at each array position. Throughout conditioning, array temperature data was collected from two J-type thermocouples adhered to the top surface of the top-plate of each array as illustrated in Figure 4.1.

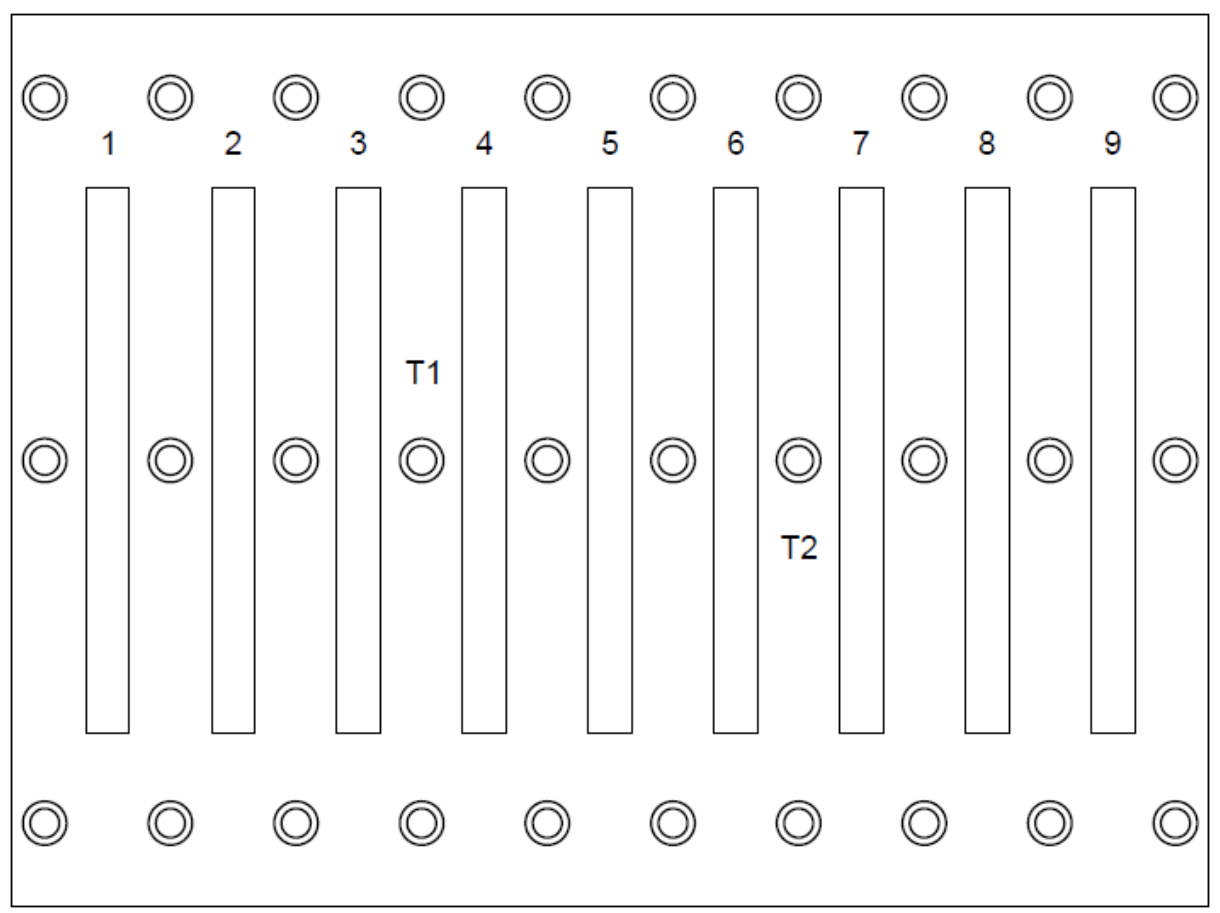

Figure 4.1: Thermocouple positions, $\mathrm{T} 1$ and $\mathrm{T} 2$, on the top-plate of conditioning arrays.

Table 4.1 presents the average and standard deviation of temperatures for each array over their conditioning duration. Each duration is represented by two sets of temperatures as two arrays were used to accommodate the 18 samples conditioned at each duration.

Shorter duration sample arrays exhibited higher average temperatures with lower standard deviation due to their position in the oven and the limited period for which the oven door was open during conditioning, but demonstrated a greater difference in average temperature between arrays. Conversely, longer conditioning durations 
Table 4.1: Array temperature distribution over conditioning durations.

\begin{tabular}{cccc}
\hline $\begin{array}{c}\text { Target } \\
\text { Duration }\end{array}$ & $\begin{array}{c}\text { Array } \\
\text { Designation }\end{array}$ & $\begin{array}{c}\text { Average } \\
\text { Temperature (C) }\end{array}$ & $\begin{array}{c}\text { Standard } \\
\text { Deviation (C) }\end{array}$ \\
\hline \multirow{2}{*}{ 1 Day $(4.9 \sqrt{\text { hours }})$} & $1(\mathrm{~A})$ & 86.85 & 0.97 \\
& $2(\mathrm{~B})$ & 80.86 & 0.71 \\
1 Week $(13.0 \sqrt{\text { hours }})$ & $4(\mathrm{~A})$ & 84.30 & 3.18 \\
& $3(\mathrm{~B})$ & 82.17 & 3.62 \\
1 Month $(26.8 \sqrt{\text { hours }})$ & $5(\mathrm{~A})$ & 78.29 & 9.77 \\
& $6(\mathrm{~B})$ & 77.69 & 10.10 \\
2 Months $(37.9 \sqrt{\text { hours }})$ & $5(\mathrm{~A})$ & 78.56 & 7.45 \\
& $6(\mathrm{~B})$ & 78.73 & 6.93 \\
3 Months $(46.5 \sqrt{\text { hours }})$ & $1 \& 3(\mathrm{~A})$ & 77.78 & 9.53 \\
\hline
\end{tabular}

yielded smaller temperature distributions between arrays but had lower average temperatures and higher standard deviation due to heat released during the opening of the oven door. From this, the average overall exposure temperature, calculate as the average array temperature over its exposure duration divided by the number of conditioned arrays, is appropriately $80.3^{\circ} \mathrm{C}, 4.7^{\circ} \mathrm{C}$ below the intend set-point temperature. As the nature of the experiment resulted in longer duration samples being subjected to lower-than-average temperatures, this may have resulted in a lower moisture absorption rate.

By comparing the temperatures of thermocouples adhered to the same conditioning array, it was determined that there was also a temperature differential across the length of the array. By linearly extrapolating the obtained temperature data, as demonstrated in Figure 4.2, the average temperature for each sample was estimated over its exposure duration. 


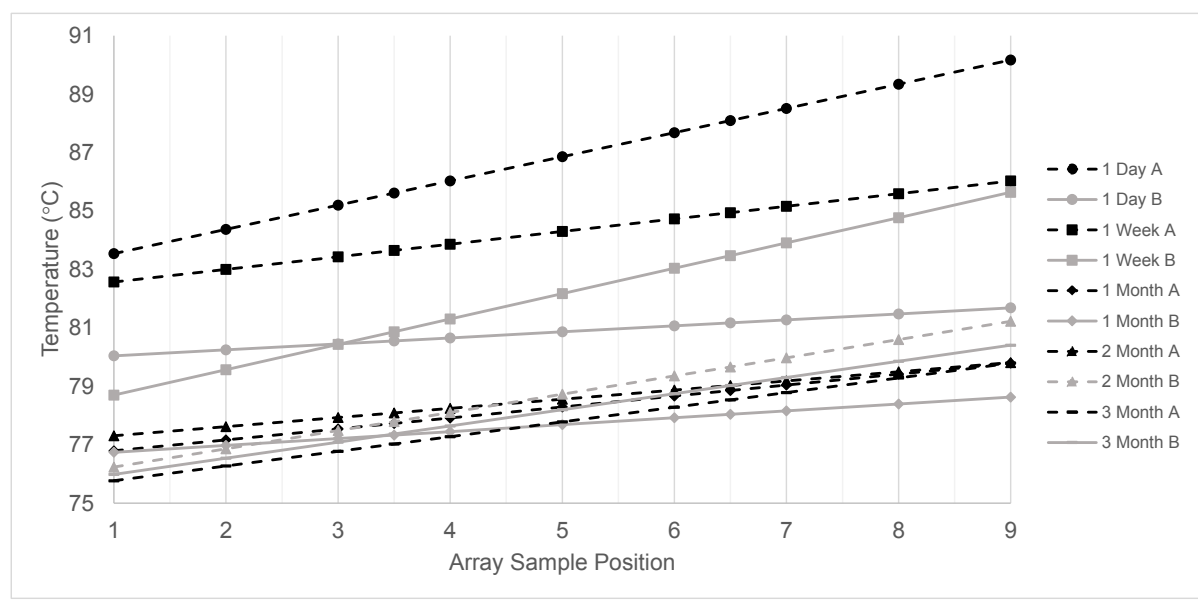

Figure 4.2: Linear extrapolation of temperature for each sample position, at all tested conditioning durations.

As discussed in Chapter 3, assuming Fickian diffusion and constant sample thickness, baseline percent moisture, percent moisture at saturation and type of diffusant, percent moisture absorbed is anticipated to increase with increasing temperature. Furthermore, with increasing exposure duration, the difference in moisture per ${ }^{\circ} \mathrm{C}$ increase will be greater. Empirically, this relationship may be validated by plotting sample percent mass change as a function of extrapolated temperature. What is anticipated is that, for a given fluid, there should be an observable increase in percent moisture absorption with increasing temperature. Moreover, with increasing conditioning duration, there should be an observable increase in the rate of change (slope) of percent moisture absorbed. Figure 4.3 shows the plots of percent change in mass as a function of extrapolated temperatures, with linear trendlines presented for each exposure duration for each fluid type.

As may be seen, there was not a consistent trend of moisture increase resulting from temperature increase for all fluids; deionized water was the only fluid which exhibited an overall increase in percent moisture absorbed. To further determine the effects of temperature distribution on flexural strength, a similar plot was generated; Figure 4.4 
Figure 4.3: Fractional change in sample mass as a function of extrapolated temperature, presented in terms of conditioning fluid and duration.

displays flexural strength as a function of extrapolated temperatures. Again, there does not appear to be a consistent trend for either fluid type or duration. While temperature is known to effect rate of moisture absorption, variations in sample composition are large enough to obscure any temperature dependent absorption trends for the tested temperature range. Hence, it may be concluded that any variances in moisture absorption, and thus flexural properties, due to a larger than specified temperature distribution are negligible for a given duration.

A number of actions may be taken to mitigate sample temperature variance in future studies. This may include purchasing an oven with a greater internal volume such that the relative difference in distance between conditioning arrays and the heating source 
may be reduced or eliminated, or purchasing multiple conditioning ovens to decrease the required load of each. Alternatively, decreasing conditioning array dimensions or heating the arrays through conduction may also be employed.

Figure 4.4: Fractional change in flexural strength as a function of extrapolated temperature, presented in terms of conditioning fluid and duration.

\subsection{Fluid Evaporation}

Periodically throughout conditioning, samples exposed to the higher volatility liquids (deionized water and ocean water) had their sample wells 'run-dry', due to an imperfect seal between adhesive tape and the conditioning array top-plate. This would result in the sample being no longer subjected to liquid exposure. Such an event may 
affect average mass change values, thus effecting interpreted moisture uptake rates and moisture at saturation values. Furthermore, a reduction in the conditioning duration could potentially result in higher flexural properties. To mitigate moisture losses, samples which ran-dry were conditioned for additional days, equivalent to the number days for which they were without liquid.

Table 4.2 compares all water-conditioned flexural sample groups - their estimated drytime (and corresponding additional days of conditioning), mass change at removal and residual strength at removal. This table shows that groups which had samples run-dry during testing demonstrated larger standard deviations in mass change values than those of continuously-conditioned groups. However, the resulting standard deviation of residual strength values associated with theses groups is not significantly greater than those of their continuously-conditioned counterparts. Moreover, there is not a strong observable trend indicating sample which ran-dry absorbed less moisture. This suggests that, similar to the comparison of moisture absorption and temperature, the relative magnitude of sample-to-sample compositional variance masks the effects of short-term dry periods over the conditioning duration. Similar SBS data is presented in Table D.1 in Appendix D. While SBS tests did not have groups with samples which ran-dry for different durations, it had two sample groups run-dry for equivalent periods due to human error. Important to note from this table is the overall smaller deviation in residual strengths compared to flexural sample groups - a result of lower void content and a test which is less sensitive to sample surface topography.

While this study did not explicitly compare ingress to egress rates, drying of the nineweek, SBS samples demonstrated that they are not equivalent for the material tested. However, compensating for samples running dry during testing by conditioning them for an equivalent number of additional days has not resulted in an overall trend of lower moisture absorption for samples dried for longer durations during conditioning. 
To limit evaporation while allowing well visibility in subsequent studies, a lid should be fabricated from a transparent material (such as polycarbonate or glass) and placed over wells containing volatile liquids during conditioning.

Table 4.2: Flexural sample groups exposed to water conditioning, comparing drytime, mass change and residual strength; standard deviations of varying-drytime groups highlighted in bold.

\begin{tabular}{|c|c|c|c|c|c|c|c|}
\hline $\begin{array}{l}\text { Sample } \\
\text { ID }\end{array}$ & $\begin{array}{l}\text { Conditioning } \\
\text { Fluid }\end{array}$ & $\begin{array}{c}\text { Intended Duration } \\
\text { (Days) }\end{array}$ & $\begin{array}{l}\text { Duration Dry } \\
\text { (Days) }\end{array}$ & $\begin{array}{c}\text { Mass } \\
\text { Change }\end{array}$ & $\begin{array}{l}\text { Mass Standard } \\
\text { Deviation }\end{array}$ & $\begin{array}{l}\text { Residual } \\
\text { Strength }\end{array}$ & $\begin{array}{c}\text { Strength Standard } \\
\text { Deviation }\end{array}$ \\
\hline FD01 & & & & $-0.069 \%$ & & $88.936 \%$ & \\
\hline FE01 & DI Water & 1 & - & $-0.063 \%$ & $0.009 \%$ & $91.842 \%$ & $2.411 \%$ \\
\hline FG01 & & & & $-0.052 \%$ & & $93.722 \%$ & \\
\hline BA09 & & & & $0.391 \%$ & & $81.626 \%$ & \\
\hline BA12 & DI Water & 7 & - & $0.257 \%$ & $0.091 \%$ & $84.338 \%$ & $4.946 \%$ \\
\hline $\mathrm{FC} 02$ & & & & $0.431 \%$ & & $74.744 \%$ & \\
\hline AA07 & & & 3 & $0.788 \%$ & & $90.407 \%$ & \\
\hline JH01 & DI Water & 30 & 2 & $0.383 \%$ & $0.352 \%$ & $100.466 \%$ & $16.818 \%$ \\
\hline JA01 & & & & $1.084 \%$ & & $67.640 \%$ & \\
\hline BB01 & & & & $1.086 \%$ & & $73.196 \%$ & \\
\hline CB05 & DI Water & 60 & - & $1.067 \%$ & $0.097 \%$ & $72.685 \%$ & $0.757 \%$ \\
\hline CB10 & & & & $1.244 \%$ & & $74.174 \%$ & \\
\hline CA11 & & & 11 & $1.862 \%$ & & $68.924 \%$ & \\
\hline $\mathrm{CA} 13$ & DI Water & 90 & 11 & $2.243 \%$ & $0.246 \%$ & $64.927 \%$ & $3.290 \%$ \\
\hline CB08 & & & 15 & $1.782 \%$ & & $71.452 \%$ & \\
\hline FF01 & & & & $0.053 \%$ & & $97.766 \%$ & \\
\hline FA03 & SO Water & 1 & - & $0.079 \%$ & $0.014 \%$ & $81.732 \%$ & $8.019 \%$ \\
\hline FB03 & & & & $0.073 \%$ & & $90.030 \%$ & \\
\hline BA03 & & & & $0.551 \%$ & & $86.995 \%$ & \\
\hline BA10 & SO Water & 7 & - & $0.697 \%$ & $0.080 \%$ & $79.846 \%$ & $3.786 \%$ \\
\hline BA11 & & & & $0.569 \%$ & & $81.259 \%$ & \\
\hline AA04 & & & - & $1.020 \%$ & & $81.259 \%$ & \\
\hline JG02 & SO Water & 30 & 2 & $0.699 \%$ & $0.206 \%$ & $85.201 \%$ & $5.081 \%$ \\
\hline JH02 & & & 1 & $0.637 \%$ & & $91.342 \%$ & \\
\hline AA13 & & & & $1.326 \%$ & & $87.803 \%$ & \\
\hline CA10 & SO Water & 60 & - & $1.270 \%$ & $0.166 \%$ & $72.126 \%$ & $7.915 \%$ \\
\hline JJ02 & & & & $1.582 \%$ & & $78.072 \%$ & \\
\hline BE01 & & & 5 & $0.911 \%$ & & $70.303 \%$ & \\
\hline $\mathrm{CA} 12$ & SO Water & 90 & 5 & $1.349 \%$ & $0.224 \%$ & $73.967 \%$ & $2.595 \%$ \\
\hline CA14 & & & - & $1.212 \%$ & & $68.952 \%$ & \\
\hline
\end{tabular}




\subsection{Gasket Moisture Uptake}

Over the first three weeks of conditioning, as proposed in section 2.1, three-month flexural samples had fluid uptake measurements performed every three days to plot moisture uptake with greater resolution through a period of predictably rapid moisture uptake and to allow for determination of saturation moisture content. However, with each measurement, the sample arrays became more difficult to reassemble due to deformation of the silicone gasket material. Figure 4.5 shows warpage observed in the gasket material.

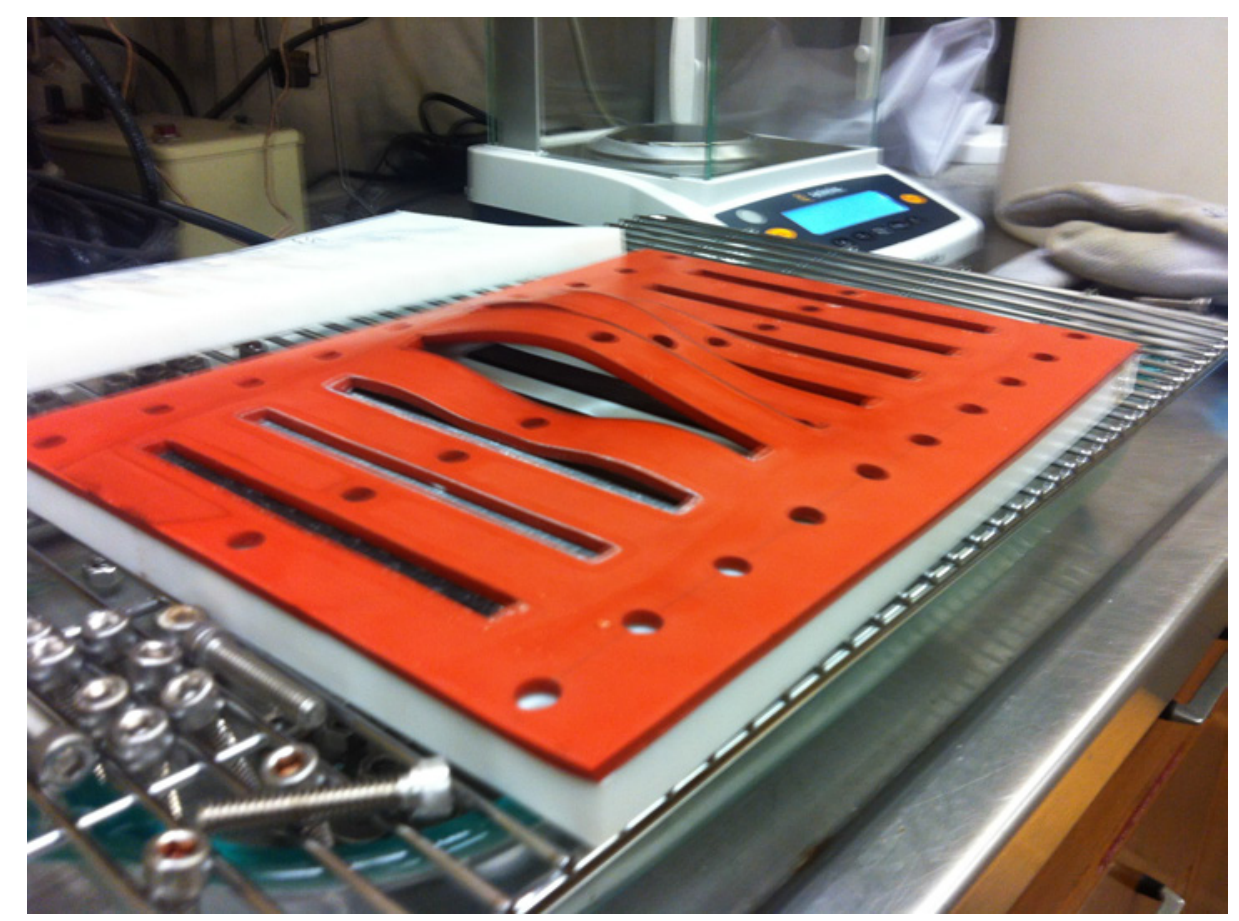

Figure 4.5: Silicone conditioning gasket following one month of exposure.

As may be seen, warpage is greatest in proximity to the central sample wells, those which contained either hydraulic fluid of turbine oil. To some degree, this was observable for all gaskets which had undergone a week or more of conditioning. Conventional silicones are composed of linear-chain Poly(dimethylsiloxane) (PDMS), illustrated in Figure 4.6. 


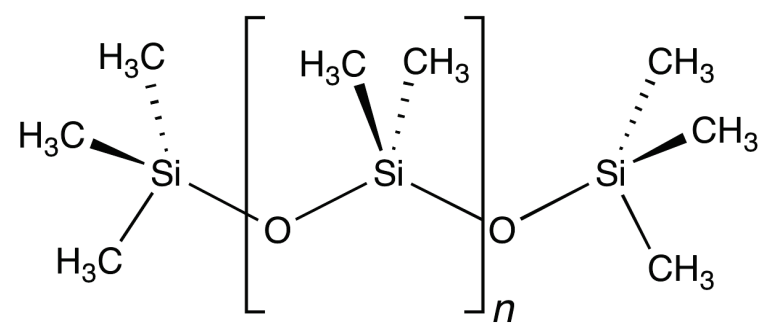

Figure 4.6: Molecular structure of Poly(dimethylsiloxane).

While, PDMS contains Si-O dipoles, outward-facing methyl side groups limit intermolecular attraction [119], hence, non-functionalized PDMS is hydrophobic in nature. As a result, favourable interaction between long-chain, nonpolar molecules of the hydraulic fluid and turbine oil fosters fluid ingress. Although preliminary evaluation of silicone as a gasket material investigated qualitative changes in hardness and observable degradation, it neglected to consider changes in volume. This localized material expansion in proximity to hydraulic fluid and turbine oil wells resulted in increasingly poorer array conformation, eventually leading to well leakage. Thus, periodic mass uptake measurements for the three-month flexural samples were halted to preserve the integrity of full duration uptake and flexural results. As saturation was not perceive over the first three weeks of exposure, it was decided that saturation moisture content would be approximated from SBS immersion testing.

To circumvent this issue for future studies, gasket materials must be selected to complement the hydrophobicity of the contained liquids. Arrays may be structured to contain like-fluids to reduce gasket mismatch and limit material requirements. 


\section{Chapter 5}

\section{Conclusions}

This work investigated the effects of environmental exposures - common to a maritime aircraft interior - on the mechanical properties of AX-5180, carbon fibre-reinforced epoxy laminate. Primary research focused on the degradation of matrix-dominant properties resulting from exposure to aerospace-specific chemicals. Prime objectives were to identify relative chemical effects, model rates of long-term chemical ingression and devise an association between mechanical degradation and moisture uptake. Research took the form of two test campaigns, investigating changes to flexural properties resulting from single-sided exposure and changes to short beam strength resulting from complete immersion. Complementary microscopy, x-ray micro-computed tomography, and thermal analysis was performed to further characterize material degradation. Shore D hardness was evaluated as a minimally invasive technique to detect the effects of moisture induced degradation. From the results obtained from primary research, it may be concluded that:

- Exposure to Royco 782 hydraulic fluid and Aeroshell turbine oil 390 at $85^{\circ} \mathrm{C}$ did not elicit a significant change in either flexural properties or short beam strength. 
- Exposure to ethylene glycol at $85^{\circ} \mathrm{C}$ caused a detectable decrease in flexural properties and significant effects over three-months of conditioning but did not show significant changes in short beam strength resulting from complete immersion.

- Exposure to deionized and ocean water showed significant degradation to both flexural properties and short beam strength with significance increasing with exposure duration.

- The presence of ocean salt in water did not demonstrate a significant effect on the measured mechanical properties over that of deionized water alone.

- Percentage change in mass plotted as a function of the square root of exposure duration produced moisture absorption curves which approximated Fickian diffusion for hydraulic fluid and turbine oil exposed samples. Water and ethylene glycol exposed samples demonstrated anomalous moisture absorption profiles.

- Accumulated water induced degradation to short beam strength was found to be a summation of reversible, chemical changes to the polymer matrix resulting from disruption to intersegmental hydrogen bonding, and irreversible, chemical changes to the polymer matrix resulting from intramolecular chain scission.

From the results of supplementary testing, it may be concluded that:

- Exposure to water induced visual degradation to exposed surfaces. Exposure to air, hydraulic fluid, turbine oil and ethylene glycol induced surface degradation, albeit less severe than water exposed samples. Surface degradation to air, hydraulic fluid and turbine oil samples was indistinguishable, while ethylene glycol exposed samples showed slightly greater void formation.

- Shore D hardness was shown to be an effective method of detecting both fluid 
ingress and mechanical failure; however, it was shown to be ineffective at quantifying fluid ingress. Further evaluation of hardness testing's ability to quantify residual properties for destructive loading is required.

- Thermogravimetric analysis showed that conditioned samples had consistent degradation temperatures with slight increase in onset of degradation attributed to exposure to water. 


\section{Chapter 6}

\section{Recommendations}

From the performed research, a number of gaps in knowledge and associated avenues for future work have been identified. Presented in this section are recommendations for future work.

\subsection{Alterations to Single-Sided Exposure Condi- tioning Fixture}

As a novel conditioning technique, a number of complications were encountered through testing, due the conditioning fixture's design and oven specifications. This included temperature variation between conditioning fixtures, fluid evaporation during testing, gasket material fluid absorption and the arduous task of removing samples from conditioning for mass evaluation. To address temperature distribution, it rec-

ommended that future work, examining single-sided exposure, procure either an oven (or ovens) which allow for a higher ratio of oven volume to conditioning fixture volume 
or decrease the volume of conditioning fixtures. To address both difficulties removing samples for mass evaluation and fluid evaporation, it is recommended that some form of high-stiffness sandwich structure, consisting of a lid and a backing plate, be clamped to the already existing conditioning fixture. Given sufficient clamping pressure, this practice will effectively eliminate the need for fasteners and prevent sample well fluid evaporation. Finally, it is recommended that future work investigating unconventional fluids first evaluate the moisture absorption characteristics of potential gasket materials used in the encasing fixture.

\subsection{Extended Single-Sided Exposure}

It was observed that single-sided flexural samples did not reach moisture saturation over a 90-day conditioning period nor did they exhibit mass loss as was observed for immersed short-beam-strength samples. Hence, it is recommended that longer duration single-side flexural conditioning be carried out-looking strictly at water as a diffusant - to investigate longer term degradation profiles and matrix material egress. Furthermore, by periodically testing both moisture infused and dried samples at equivalent intervals, a better understanding of intermolecular and intramolecular contribution to net degradation as a function of exposure duration may be gained.

\subsection{Chemical Analysis}

Through conditioning, a number of anomalous observations were made. Namely, white precipitate formation in deionized water exposed samples, precipitate formation on short-beam-strength sample following drying and colour change of ethylene glycol resulting from interactions with the composite. Thus, it is recommended that future 
work investigates the chemical composition of amassed precipitate and attempts to isolate and analyzed ethylene glycol's colourant. Chemical analysis may include, but is not limited to, Fourier transform infrared spectroscopy, high-performance liquid chromatography, and x-ray photoelectron spectroscopy.

\subsection{Alternative Chemical Exposure}

Experimentation demonstrated that water contributed to significant degradation of the investigated fibre-reinforced epoxy. This correlation was believed to be a result of water's polarity, allowing it to disrupt both epoxy's intermolecular and intramolecular bonding network. Moving forward, it recommended that future work verifies this inference by investigating the degradative effects of similarly polar solvents such as propanol, butanol and dimethyl sulfoxide. Furthermore, it is recommended that a similar investigation looks at the absorption rate and degradation induced by conditioning with a surfactant mixed with water - representative of a cleaning mixture used in service.

\subsection{Cyclic Exposure}

Water conditioned samples showed the egress of constituent composite material into the surrounding solution. From this, it is recommended that future work investigates the effects of cyclic exposure by removing samples form conditioning, drying them and re-immersing them in a water free of leachant. This method both, serves as a more realistic representation of composite fluid exposure in an in-service scenario and allows for determination of the effects of leachant concentration on progressive degradation and saturation. 


\subsection{Tomographic Analysis of SBS Samples}

Results of x-ray micro-computed tomography scans demonstrated an insignificant effect of ingressed water on sample void volume. However, subsequential short-beamstrength sample mass analysis demonstrated significant mass loss following nine weeks of immersion and drying. Hence, it is recommended that nine-week, short-beamstrength samples be subjected to tomographic analysis to better characterize observed material loss.

\subsection{Profilometry}

X-ray micro-computed tomography demonstrated itself to be an effective means of evaluating internal composite structure. However, it proved to be inadequate for evaluating changes to surface structure. Consequently, it is recommended that future work analyzing composite surface degradation investigates profilometry as a potential characterization technique.

\subsection{Synergistic Effects of Impact}

As indicated by secondary research, barely visible impact damage is an environmental factor with the propensity to significantly reduce mechanical properties [120,121]. Thus, it is recommended that future work examines the synergistic effects of exposure to water and impact on residual properties. Studies of this nature may investigate both impact post-conditioning, probing the effects of water ingress on impact damage, and conditioning post-impact, probing the effects of impact damage on rate of ingress. 


\section{References}

[1] M. Smith, "Material Safety Data Sheet - AX-5180," tech. rep., Axiom Materials Inc., Santa Ana, 2012.

[2] H. M. Cooper, "Organizing knowledge synthesis: a taxonomy of literature reviews," Knowledge in society, vol. 1, no. 1, pp. 104-126, 1988.

[3] Federal Aviation Administration, "Composite Aircraft Structure AC20-107B," tech. rep., U.S. Department of Transportation, 2009.

[4] W. L. Hawkins, B. T. Laboratories, and H. Murray, "Thermal and Oxidative Degradation," Polymer Engineering \& Science, vol. 4, no. 3, pp. 187-192, 1964.

[5] A. Chateauminois, L. Vincent, B. Chabert, and J. P. Soulier, "Study of the Interfacial Degradation of a Glass Epoxy Composite During Hygrothermal Aging Using Water Diffusion Measurements and Dynamic-Mechanical ThermalAnalysis," Polymer, vol. 35, no. 22, pp. 4766-4779, 1994.

[6] Y. J. Weitsman, "Composites in the Sea : Sorption, Strength and Fatigue," in ICCM, (Paris), pp. 2-9, 1999.

[7] D. Powers, "Interaction of Water with Epoxy," tech. rep., Sandia National Laboratories, Albuquerque, NM, 2009.

[8] K. Armstrong, W. Cole, and G. Bevan, Care and Repair of Advanced Composites, vol. 2nd. SAE, 2005.

[9] T. C. Jester and Getty Conservation Institute, Twentieth-century building materials : history and conservation. Getty Publications, 1995.

[10] U.S. Department of Transportation Federal Aviation Administration, "Overview Title 14 of the Code of Federal Regulations (14 CFR)," tech. rep., U.S. Department of Transportation Federal Aviation Administration, 2016. 
[11] Society of Automotive Engineers. and National Institute for Aviation Research (U.S.), Composite Materials Handbook-17. Volume 1 - Polymer Matrix Composites - Guidelines for Characterization of Structural Materials. SAE International, 2012.

[12] U.S. Department of Transportation Federal Aviation Administration, "Title 14 of the Code of Federal Regulation Part 23 - Airwothiness Standards: Normal, Utility, Acrobatic, and Commuter Category Airplanes," tech. rep., U.S. Department of Transportation Federal Aviation Administration, 2008.

[13] R. Brown, D. Kockott, and P. Trubiroha, "A Review of Accelerated Durability Tests," Tech. Rep. 18, VAMAS, 1995.

[14] T. K. Tsotsis, "Thermo-Oxidative Aging of Composite Materials," Journal of Composite Materials, vol. 29, pp. 410-422, feb 1995.

[15] D. C. Montgomery, Design and analysis of experiments. Hoboken, NJ: John Wiley \& Sons, Inc, 2013.

[16] D. C. Montgomery, Applied Statistics and Probability for Engineers Third Edition, vol. 37. John Wiley \& Sons, Inc., 3rd ed., 2003.

[17] R. A. Fisher, Statistical Methods for Research Workers. Edinburgh: Oliver and Boyd Ltd., fifth ed., 1925.

[18] R. A. Fisher, "The Arrangement of Field Experiments," in Breakthroughs in Statisitcs (S. Kotz and N. L. Johnson, eds.), pp. 82-91, New York, NY: SpringerVerlag, 1992.

[19] C. W. Dunnett, "A Multiple Comparison Procedure for Comparing Several Treatments with a Control," Journal of the American Statistical Association, vol. 50, p. 1096, dec 1955.

[20] J. J. Randolph, "A Guide to Writing the Dissertation Literature Review," Practical Assessment, Research \& Evaluation, vol. 14, no. 13, pp. 1-13, 2009.

[21] P. Robinson, E. Greenhalgh, and S. Pinho, Failure Mechanisms in Polymer Matrix Composites. Cambridge: Woodhead Publishing Limited, 1st ed., 2012.

[22] United States Department of Defense, "MIL-STD-810G Test Method Standard Environmental Engineering Considerations and Laboratory Tests," 2008. 
[23] J. L. Bolland, "Kinetics of Olefin Oxidation," Quarterly Reviews, vol. 3, no. 1, pp. 1-21, 1949.

[24] S. Qian, T. Igarashi, and K. H. Nitta, "Thermal degradation behavior of polypropylene in the melt state: Molecular weight distribution changes and chain scission mechanism," Polymer Bulletin, vol. 67, no. 8, pp. 1661-1670, 2011.

[25] M. Akay, G. R. Spratt, and B. Meenan, "The effects of long-term exposure to high temperatures on the ILSS and impact performance of carbon fibre reinforced bismaleimide," Composites Science and Technology, vol. 63, no. 7, pp. 1053-1059, 2003.

[26] M. Akay and G. R. Spratt, "Evaluation of thermal ageing of a carbon fibre reinforced bismalemide," Composites Science and Technology, vol. 68, no. 1516, pp. 3081-3086, 2008.

[27] J. Wolfrum, S. Eibl, and L. Lietch, "Rapid evaluation of long-term thermal degradation of carbon fibre epoxy composites," Composites Science and Technology, vol. 69, no. 3-4, pp. 523-530, 2009.

[28] P. Hutapea and F. Yuan, "The effect of thermal aging on the Mode-I interlaminar fracture behavior of a high-temperature IM7/LaRC-RP46 composite," Composites Science and Technology, vol. 59, pp. 1271-1286, jun 1999.

[29] A. D. Adamczak, A. A. Spriggs, D. M. Fitch, W. Awad, C. A. Wilkie, and J. C. Grunlan, "Thermal degradation of high-temperature fluorinated polyimide and its carbon fiber composite," Journal of Applied Polymer Science, vol. 115, no. 4, pp. 2254-2261, 2010.

[30] N. L. Batista, M. L. Costa, K. Iha, and E. C. Botelho, "Thermal degradation and lifetime estimation of poly(ether imide)/carbon fiber composites," Journal of Thermoplastic Composite Materials, vol. 28, no. 2, pp. 265-274, 2015.

[31] B. Dao, J. Hodgkin, J. Krstina, J. Mardel, and W. Tian, "Accelerated aging versus realistic aging in aerospace composite materials. I. The chemistry of thermal aging in a low-temperature-cure epoxy composite," Journal of Applied Polymer Science, vol. 102, no. 5, pp. 4291-4303, 2006.

[32] B. G. Kumar, R. P. Singh, and T. Nakamura, "Degradation of Carbon Fiberreinforced Epoxy Composites by Ultraviolet Radiation and Condensation," Journal of Composite Materials, vol. 36, no. 24, pp. 2713-2733, 2002. 
[33] T. Nakamura, R. P. Singh, and P. Vaddadi, "Effects of environmental degradation on flexural failure strength of fiber reinforced composites," Experimental Mechanics, vol. 46, no. 2, pp. 257-268, 2006.

[34] E. Guzmán, J. Cugnoni, and T. Gmür, "Multi-factorial models of a carbon fibre/epoxy composite subjected to accelerated environmental ageing," Composite Structures, vol. 111, no. 1, pp. 179-192, 2014.

[35] Axiom Materials Inc., "AX-5180 Technical Data Sheet," tech. rep., Axiom Materials Inc., Santa Ana, 2012.

[36] A. F. Couture, "Effects of Moisture, Salinity and Temperature on Mode I Fracture Toughness of Adhesively-Bonded Composites," Master's thesis, Carleton University, 2011.

[37] A. Chatterjee and J. W. Gillespie, "Moisture absorption behavior of epoxies and their S2 glass composites," Journal of Applied Polymer Science, vol. 108, pp. 3942-3951, jun 2008.

[38] G. Z. Xiao and M. E. R. Shanahan, "Irreversible Effects of Hygrothermal Aging on DGEBA/DDA Epoxy Resin," Journal of Applied Polymer Science, vol. 69, no. 2, pp. 363-369, 1998.

[39] D. Colombini, J. Martinez-Vega, and G. Merle, "Dynamic mechanical investigations of the effects of water sorption and physical ageing on an epoxy resin system," Polymer, vol. 43, no. 16, pp. 4479-4485, 2002.

[40] T. Rockett and V. Rose, "The Cause of Boat Hull Blisters," tech. rep., University of Rhode Island, Kingston, 1987.

[41] J. Komorowski and S. Beland, "Moisture Diffusion in Graphite \& BismalimideModified-Epoxy Composite," Canadian Aeronautics and Space Journal, vol. 31, no. 3, pp. 218-226, 1986.

[42] R. Wang, L. Jiang, W. Liu, and J. Lv, "Study on Hygrothermal Properties of Carbon Fiber Reinforced Composites Aged in Cyclic Environment," Polymers and Polymer Composites, vol. 19, no. 4, pp. 313 - 317, 2011.

[43] V. La Saponara, "Environmental and chemical degradation of carbon/epoxy and structural adhesive for aerospace applications: Fickian and anomalous diffusion, Arrhenius kinetics," Composite Structures, vol. 93, no. 9, pp. 2180-2195, 2011. 
[44] Z. Youssef, F. Jacquemin, D. Gloaguen, and R. Guillen, "A multi-scale analysis of composite structures: Application to the design of accelerated hygrothermal cycles," Composite Structures, vol. 82, no. 2, pp. 302-309, 2008.

[45] A. C. Loos and G. S. Springer, "Moisture Absorption of Graphite-Epoxy Composites Immersed in Liquids and in Humid Air," J Compos Mater, vol. 13, pp. 131-147, 1979.

[46] R. D. Adams and M. M. Singh, "The Dynamic Properties of Fibre-Reinforced Polymers Exposed to Hot, Wet Conditions," 1996.

[47] L. Prian and A. Barkatt, "Degradation Mechanism of Fiber-Reinforced Plastics and its Implications to Prediction of Long-Term Behavior," Journal of Materials Science, vol. 34, no. 16, pp. 3977-3989, 1999.

[48] Y. J. Weitsman, "Anomalous fluid sorption in polymeric composites and its relation to fluid-induced damage," Composites Part A: Applied Science and Manufacturing, vol. 37, no. 4, pp. 617-623, 2006.

[49] H. G. Carter and K. G. Kibler, "Langmuir-Type Model for Anomalous Moisture Diffusion In Composite Resins," Journal of Composite Materials, vol. 12, pp. 118-131, jul 1978.

[50] M. H. Shirangi and B. Michel, Moisture Sensitivity of Plastic Packages of IC Devices. Springer US, 2010.

[51] R. Selzer and K. Friedrich, "Influence of water up-take on interlaminar fracture properties of carbon fibre-reinforced polymer composites," Journal of Materials Science, vol. 30, pp. 334-338, 1995.

[52] A. Garg and O. Ishai, "Hygrothermal influence on delamination behavior of graphite/epoxy laminates," Engineering Fracture Mechanics, vol. 22, no. 3, pp. 413-427, 1985.

[53] L. Asp, "The effects of moisture and temperature on the interlaminar delamination toughness of a carbon/epoxy composite," Composites Science and Technology, vol. 58, pp. 967-977, jan 1998.

[54] M. Todo, T. Nakamura, and K. Takahashi, "Effects of Moisture Absorption on the Dynamic Interlaminar Fracture Toughness of Carbon/Epoxy Composites," Journal of Composite Materials, vol. 34, pp. 630-648, apr 2000. 
[55] W. Bradley and T. Grant, "The effect of the moisture absorption on the interfacial strength of polymeric matrix composites," Journal of materials science, vol. 30, pp. 5537-5542, 1995.

[56] Y. Zhong and S. C. Joshi, "Impact behavior and damage characteristics of hygrothermally conditioned carbon epoxy composite laminates," Materials and Design, vol. 65, pp. 254-264, 2015.

[57] E. Woldesenbet, N. Gupta, and J. R. Vinson, "Determination of moisture effects on impact properties of composite materials," Journal of Materials Science, vol. 37, no. 13, pp. 2693-2698, 2002.

[58] A. Siriruk and D. Penumadu, "Degradation in fatigue behavior of carbon fibervinyl ester based composites due to sea environment," Composites Part B: Engineering, vol. 61, pp. 94-98, 2014.

[59] L. V. Smith and Y. J. Weitsman, "The immersed fatigue response of polymer composites," International Journal of Fracture, vol. 82, no. 1, pp. 31-42, 1990.

[60] United States Department of Defense, "Composite Materials Handbook Vol. 3: Polymer Matrix Composites Materials Usage, Design, and Analysis," Tech. Rep. F, United States Department of Defense, 2002.

[61] R. J. Morgan, J. E. O’Neal, and D. L. Fanter, "The effect of moisture on the physical and mechanical integrity of epoxies," Journal of Materials Science, vol. 15 , no. 6, pp. 751-764, 1980.

[62] H. S. Choi, K. J. Ahn, J. Nam, and H. J. Chun, "Hygroscopic aspects of epoxy / carbon fiber composite laminates in aircraft environments," Composites, vol. 32, no. Part A, pp. 709-720, 2001.

[63] V. N. Kanellopoulos, G. H. Wostenholm, and B. Yates, "The absorption of fluids by reinforced and unreinforced resins," Journal of Materials Science, vol. 20, pp. 2503-2511, jul 1985.

[64] Y. Sugita, C. Winkelmann, and V. La Saponara, "Environmental and chemical degradation of carbon/epoxy lap joints for aerospace applications, and effects on their mechanical performance," Composites Science and Technology, vol. 70, no. 5, pp. 829-839, 2010. 
[65] R. A. Campbell, B. M. Pickett, V. L. Saponara, and D. Dierdorf, "Thermal Characterization and Flammability of Structural Epoxy Adhesive and Carbon/Epoxy Composite with Environmental and Chemical Degradation," Journal of Adhesion Science and Technology, vol. 26, no. 7, pp. 889-910, 2012.

[66] B. Landry, G. Laplante, and L. R. Leblanc, "Environmental effects on mode II fatigue delamination growth in an aerospace grade carbon/epoxy composite," Composites Part A: Applied Science and Manufacturing, vol. 43, no. 3, pp. 475485, 2012.

[67] J. D'Almeida, "Effects of distilled water and saline solution on the interlaminar shear strength of an aramid/epoxy composite," Composite Technologies for 2020, vol. 22, no. 6, pp. 448-450, 1991.

[68] W. A. de Morais, S. N. Monteiro, and J. R. M. D'Almeida, "Evaluation of repeated low energy impact damage in carbon-epoxy composite materials," Composite Structures, vol. 67, no. 3 SPEC.ISS., pp. 307-315, 2005.

[69] P. O. Sjoblom, J. T. Hartness, and T. M. Cordell, "On Low-Velocity Impact Testing of Composite Materials," Journal of Composite Materials, vol. 22, pp. 30-52, jan 1988.

[70] B. P. Jang, W. Kowbel, and B. Z. Jang, "Impact behavior and impact-fatigue testing of polymer composites," Composites Science and Technology, vol. 44, no. 2, pp. 107-118, 1992.

[71] D. R. Ambur, J. H. Starnes, and C. B. Prasad, "Low-speed impact damageinitiation characteristics of selected laminated composite plates," AIAA Journal, vol. 33, no. 10, pp. 1919-1925, 1995.

[72] D. Whisler, "Low velocity blunt impacts on composite aircraft structures," Master's thesis, UC San Diego, 2009.

[73] G. Li, S.-s. Pang, J. E. Helms, and S. I. Ibekwe, "Low velocity impact response of GFRP laminates subjected to cycling moistures," Polymer Composites, vol. 21, no. 5, pp. 686-695, 2000.

[74] W. Johnson, J. Masters, M. Karasek, L. Strait, M. Amateau, and J. Runt, "Effect of Temperature and Moisture on the Impact Behavior of Graphite/Epoxy Composites: Part IImpact Energy Absorption," Journal of Composites Technology and Research, vol. 17, p. 3, jan 1995. 
[75] C.-c. Ma, N. H. Tai, S. H. Wu, S. H. Lin, and J. F. Wu, "The Hygrothermal Effect on the Fatigue Behavior of Carbon Fiber / Peek Laminate Composites ( I )," Journal of Polymer Research, vol. 1, no. 1, pp. 17-24, 1994.

[76] A. Malhotra, F. J. Guild, and M. J. Pavier, "Edge impact to composite laminates: Experiments and simulations," Journal of Materials Science, vol. 43, no. 20, pp. 6661-6667, 2008.

[77] A. Malhotra and F. J. Guild, "Impact damage to composite laminates: Effect of impact location," Applied Composite Materials, vol. 21, no. 1, pp. 165-177, 2014

[78] A. T. Rhead, D. Marchant, and R. Butler, "Compressive strength of composite laminates following free edge impact," Composites Part A: Applied Science and Manufacturing, vol. 41, no. 9, pp. 1056-1065, 2010.

[79] A. Hodzic, J. K. Kim, and Z. H. Stachurski, "Nano-indentation and nanoscratch of polymer/glass interfaces. II: Model of interphases in water aged composite materials," Polymer, vol. 42, no. 13, pp. 5701-5710, 2001.

[80] A. A. Wazzan, H. A. Al-Turaif, and M. A. Daous, "Influence of KOH solution on the tensile strength and chemical stability of carbon fiber-reinforced epoxy resin composites," Journal of Applied Polymer Science, vol. 96, no. 5, pp. 1682-1690, 2005 .

[81] N. ElAgamy, J. Laliberte, and F. Gaidies, "Quantitative analysis of fatigue cracks in laminated carbon fibre-reinforced polymer composites using microcomputed tomography," Journal of Composite Materials, no. October, 2015.

[82] S. Sethi and B. Ray, "Evaluation of structural integrity and mechanical behavior of advanced FRP composites," International Journal of Structural Integrity, vol. 2, no. 2, pp. 214-222, 2011.

[83] J.-K. Kim, M.-L. Sham, and J. Wu, "Nanoscale characterisation of interphase in silane treated glass fibre composites," Composites Part A: Applied Science and Manufacturing, vol. 32, no. 5, pp. 607-618, 2001.

[84] P. Frontini, S. Lotfian, M. A. Monclus, and J. M. Molina-Aldareguia, "High Temperature Nanoindentation Response of RTM6 Epoxy Resin at Different Strain Rates," Experimental Mechanics, pp. 851-862, 2015. 
[85] ASTM International, "ASTM Standard D790-15e2, Standard Test Methods for Flexural Properties of Unreinforced and Reinforced Plastics and Electrical Insulating Materials," 2015.

[86] ASTM International, "ASTM Standard D7264/D7264M-15, Standard Test Method for Flexural Properties of Polymer Matrix Composite Materials," 2015.

[87] ASTM International, "ASTM Standard D6272-10, Standard Test Method for Flexural Properties of Unreinforced and Reinforced Plastics and Electrical Insulating Materials by Four-Point Bending," 2010.

[88] ASTM International, "ASTM Standard D2344/D2344M-13, Standard Test Method for Short-Beam Strength of Polymer Matrix Composite Materials and Their Laminates," 2013.

[89] D. F. Adams and J. M. Busse, "Suggested Modifications of the Short Beam Shear Test Method," in 49th International SAMPE Symposium and Exhibition, (Long Beach, CA), pp. 707 - 719, 2004.

[90] F. I. Baratta, "Requirements for Flexure Testing of Brittle Materials," tech. rep., Army Materials and Mechanics Research Center, Alexandria, VA, 1982.

[91] ASTM International, "ASTM Standard D5379/D5379M-12, Standard Test Method for Shear Properties of Composite Materials by the V-Notched," 2012.

[92] ASTM International, "ASTM Standard D7078/7078M-12, Standard Test Method for Shear Properties of Composite Materials by V-Notched Rail," 2012.

[93] J. R. Davis and ASM International. Handbook Committee., Metals Handbook, Desk Edition. ASM International, 2nd ed., 1998.

[94] K. G. Hoge, "Influence of Strain Rate on Mechanical Properties of 6061-T6 Aluminum under Uniaxial and Biaxial States of Stress properties of materials under dynamic tensile loads," Experimental Mechanics, vol. 6, no. 4, pp. 204$211,1967$.

[95] ASTM International, "ASTM Standard D5229/D5229M-14, Standard Test Method for Moisture Absorption Properties and Equilibrium Conditioning of Polymer Matrix Composite Materials," 2014.

[96] Cole-Parmer Instrument Company LLC., "Chemical Compatibility Database." https://www. coleparmer.ca/Chemical-Resistance. [Accessed: 2017-05-01]. 
[97] Eastman Chemcial Company, "AF-9 Skydrol Overview." http://www . eastman.com/Literature_Center/A/AF9.pdf. [Accessed: 2017-05-01].

[98] Moss Rubber and Equipment Corporation, "Chemical Resistance Chart." http://www.mossrubber.com/pdfs/Chem_Res.pdf. [Accessed: 2017-05-01].

[99] Mykin Inc., "Rubber Chemical Resistance Chart." http://mykin.com/ rubber-chemical-resistance-chart. [Accessed: 2017-05-01].

[100] W. Jost, "The Fundamental Laws of Diffusion," in Diffusion in Solids, Liquids, Gases, ch. 1, pp. 1-42, New York: Academic Press Inc., third ed., 1952.

[101] C. Shen and G. S. Springer, "Moisture Absorption and Desorption of Composite Materials," Journal of Composite Materials, vol. 10, pp. 2-20, 1976.

[102] C. D. Shirrell, "Diffusion of water vapor in graphite/epoxy composites," $A d$ vanced Composite Materials-Environmental Effects, 1978.

[103] S. A. Grammatikos, B. Zafari, M. C. Evernden, J. T. Mottram, and J. M. Mitchels, "Moisture uptake characteristics of a pultruded fibre reinforced polymer flat sheet subjected to hot/wet aging," Polymer Degradation and Stability, vol. 121, pp. 407-419, 2015.

[104] C. Shirrell and J. Halpin, "Moisture Absorption and Desorption in Epoxy Composite Laminates," in Composite Materials: Testing and Design, pp. 514-528, American Sodety for Testing and Material, 1977.

[105] M. D. Gilbert, Mechanism and Kinetics of the Dicyandiamide Cure of Epoxy Resins. Doctoral dissertations, University of Massachusetts Amherst, 1988.

[106] Y. Wang, C. Zhou, Q. Zhang, and Z. Huang, "Synthesis of Fumed Silica Treated with Organosilane and Its Effect on Epoxy Resin," Polymer-Plastics Technology and Engineering, vol. 52, no. 2, pp. 145-148, 2013.

[107] Product Safety - Anderol Specialty Lubricants, "Royco 782 Synthetic Hydraulic Fluid Safety Data Sheet," Tech. Rep. 1, Anderol Specialty Lubricants, a division of Chemtura Corporation, East Hanover, NJ, 2015.

[108] G. Z. Xiao, G. Z. Xiao, M. Delamar, M. Delamar, M. E. R. Shanahan, and M. E. R. Shanahan, "Irreversible interactions between water and DGEBA/DDA epoxy resin during hygrothermal aging," Journal of Applied Polymer Science, vol. 65, no. 3, pp. 449-458, 1997. 
[109] Y. Zhong and S. C. Joshi, "Impact behavior and damage characteristics of hygrothermally conditioned carbon epoxy composite laminates," Materials and Design, vol. 65, pp. 254-264, 2015.

[110] A. I. Vogel and B. S. Furniss, Vogel's Textbook of Practical Organic Chemistry. Longman Scientific \& Technical, 5th ed., 1989.

[111] J. M. Augl and A. E. Berger, "The Effect of Moisture on Carbon Fiber Reinforced Epoxy Composites. 1. Diffusion," tech. rep., Naval Surface Waepons Center, White Oak Lab, Silver Springs, MD, 1976.

[112] G. Bouvet, N. Dang, S. Cohendoz, X. Feaugas, S. Mallarino, and S. Touzain, "Impact of polar groups concentration and free volume on water sorption in model epoxy free films and coatings," Progress in Organic Coatings, vol. 96, no. February, pp. 32-41, 2015.

[113] M. J. Adamson, "Thermal expansion and swelling of cured epoxy resin used in graphite/epoxy composite materials," Journal of Materials Science, vol. 15, no. 7 , pp. $1736-1745,1980$.

[114] Eastman Chemical Company, "Skydrol 500B-4 Fire Resistant Hydraulic Fluid Safety Data Sheet," tech. rep., Eastman Chemcial Company, Kingsport, TN, 2015.

[115] ALBA Sh.A, "AeroShell Fluid 41 Material Safety Data Sheet," tech. rep., Royal Dutch Shell plc, 2014.

[116] ASTM International, "ASTM Standard D2240-15, Standard Test Method for Rubber Property-Durometer Hardness," 2015.

[117] ASTM International, "ASTM E1356-08, Standard Test Method for Assignment of the Glass Transition Temperatures by Differential Scanning Calorimetry," 2008 .

[118] ASTM International, "ASTM E1131-08, Standard Test Method for Compositional Analysis by Thermogravimetry," 2008.

[119] M. Andriot, J. V. Degroot, R. Meeks, E. Gerlach, M. Jungk, a. T. Wolf, S. Cray, T. Easton, a. Mountney, S. Leadley, S. H. Chao, a. Colas, F. de Buyl, a. Dupont, J. L. Garaud, F. Gubbels, J. P. Lecomte, B. Lenoble, S. Stassen, C. Stevens, $\mathrm{X}$. Thomas, and G. Shearer, "Silicones in Industrial Applications," Inorganic Polymers, pp. 61-161, 2007. 
[120] H. Kan, R. Cordero, and R. Whitehead, "Advanced Certification Methodology for Composite Structures," tech. rep., Northrop Corporation, Hawthorne, CA, 1997.

[121] E. Demuts, R. S. Whitehead, and R. B. Deo, "Assesment of Damage Tolerance in Composites," Composite structures, vol. 4, no. 1, pp. 45-58, 1985.

[122] Canadian Centre for Occupational Health and Safety, "Ultraviolet Radiation." Available: http://www.ccohs.ca/oshanswers/phys_agents/ ultravioletradiation.html, 2016. [Accessed: 2017-08-05].

[123] D. Roylance and M. Roylance, "Weathering of fiber-reinforced epoxy composites," Polymer Engineering and Science, vol. 18, no. 4, pp. 249-254, 1978.

[124] M. M. Shokrieh and A. Bayat, "Effects of Ultraviolet Radiation on Mechanical Properties of Glass/Polyester Composites," Journal of Composite Materials, vol. 41, no. 20, pp. 2443-2455, 2007.

[125] J. D. Gu, "Microbiological deterioration and degradation of synthetic polymeric materials: Recent research advances," International Biodeterioration and Biodegradation, vol. 52, no. 2, pp. 69-91, 2003.

[126] C. Tascioglu, B. Goodell, R. Lopez-Anido, M. Peterson, W. Halteman, and J. Jellison, "Monitoring fungal degradation of E-glass/phenolic fiber reinforced polymer (FRP) composites used in wood reinforcement," International Biodeterioration and Biodegradation, vol. 51, no. 3, pp. 157-165, 2003.

[127] J. D. Gu, C. Lu, K. Thorp, a. Crasto, and R. Mitchell, "Fiber-reinforced polymeric composites are susceptible to microbial degradation.," Journal of industrial microbiology \& biotechnology, vol. 18, no. 6, pp. 364-9, 1997.

[128] J.-d. Gu, T. Ford, K. Thorp, and R. Mitchell, "Microbial Growth on Fiber Reinforced Composite Materials *," 1996.

[129] P. A. Wagner, B. J. Little, K. R. Hart, and R. I. Ray, "Biodegradation of Composite Materials," International Biodeterioration $\&$ Biodegradation, vol. 38, no. 2, pp. 125-132, 1996.

[130] W. C. Tucker and R. Brown, "Blister Formation on Graphite / Polymer Composites Galvanically Coupled with Steel in Seawater," tech. rep., University of Rhode Island, 1988. 
[131] F. E. Sloan and J. B. Talbot, "Corrosion of Graphite-Fiber-Reinforced Composites IIAnodic Polarization Damage," Corrosion, vol. 48, pp. 1020-1026, dec 1992.

[132] M. Tavakkolizadeh and H. Saadatmanesh, "Galvanic Corrosion of Carbon and Steel in Aggressive Environments," Journal of Composites for Construction, vol. 5, pp. 200-210, aug 2001. 


\section{Appendix A}

\section{Extended Background and Literature Review}

This appendix details supplementary background information and secondary research to that which has been presented in Section 1 of the main thesis body.

\section{A.1 Supplementary Background}

Equations A.1 through A.4 describe the total SS, SS of effect A, SS of effect B and SS of effect A and B's interaction, respectively. From this, with theses values know, rearrangement of Equation 1.9 allows from algebraic determination of the SS of error, as shown in A.5; $y_{\ldots}^{2}$ represents the sum of all observations.

$$
S S_{T}=\sum_{i=1}^{a} \sum_{j=1}^{b} \sum_{k=1}^{n} y_{i j k}^{2}-\frac{y_{\ldots}^{2}}{a b n}
$$




$$
\begin{gathered}
S S_{A}=\frac{1}{b n} \sum_{i=1}^{a} \sum_{k=1}^{n} y_{i}^{2}-\frac{y_{\ldots}^{2}}{a b n} \\
S S_{B}=\frac{1}{a n} \sum_{j=1}^{b} y_{j}^{2}-\frac{y_{\ldots}^{2}}{a b n} \\
S S_{A B}=\frac{1}{n} \sum_{i=1}^{a} \sum_{j=1}^{b} y_{i j}^{2}-\frac{y_{\ldots}^{2}}{a b n}-S S_{A}-S S_{B} \\
S S_{E}=S S_{T}-S S_{A}-S S_{B}-S S_{A B}
\end{gathered}
$$

\begin{tabular}{|c|c|}
\hline Characteristic & Categories \\
\hline \multirow{4}{*}{ Focus } & Research outcomes \\
\hline & Research methods \\
\hline & Theories \\
\hline & Practices or applications \\
\hline \multirow{6}{*}{ Goal } & Integration \\
\hline & (a) Generalization \\
\hline & (b) Conflict resolution \\
\hline & (c) Linguistic bridge-building \\
\hline & Criticism \\
\hline & Identification of central issues \\
\hline \multirow{2}{*}{ Perspective } & Neutral representation \\
\hline & Espousal of position \\
\hline \multirow{3}{*}{ Coverage } & Exhaustive \\
\hline & Exhaustive with selective citation \\
\hline & Representative \\
\hline
\end{tabular}

Table A.1: Cooper's taxonomy of literature reviews with characteristics pertinent to the study highlighted in bold [2]. 
Central or pivotal

\begin{tabular}{ll}
\hline & Historical \\
Organization & Conceptual \\
& Methodological \\
\hline & Specialized scholars \\
& General scholars \\
Audience & Practitioners or policymakers \\
& General public \\
\hline
\end{tabular}

Table A.2: List of extracted codebook categories.

\section{Category}

Title

Publication Year

Material

- Matrix Resin

- Fibre Type

- Orientation/lay-up

- Core material

- Core Structure

Manufacturing Technique

Degradation Type and Source

Exposure Timescale

Evaluation Method

Quantifiable Metric

Results

- Qualitative

- Quantitative

Number of References

Additional Notes 


\section{A.2 Supplementary Literature Review}

\section{A.2.1 Ultraviolet Radiation}

Ultraviolet (UV) radiation is electromagnetic radiation possessing a wavelength between 290 and $380 \mathrm{~nm}$ [21]. UV radiation can be emitted by a number of high energy sources, including the sun, however, it can also be emitted by man-made sources including black lamps, fluorescent equipment, hydrogen lamps, metal halide lamps, mercury lamps and welding equipment [122]. Electromagnetic radiation is produced as an atomic emission resulting from an electron transitioning from a high energy state to a lower energy state. The difference in potential energy between the two states dictates the resulting energy, and thus the wavelength of the resulting photon, through the equation

$$
\Delta E=\frac{h c}{\lambda}
$$

where $\Delta E$ corresponds to the electron's change in energy, $h$ is Planck's constant, $c$ is the speed of light and $\lambda$ is the radiant photon's wavelength. Conversely, when a photon is absorbed by an electron, the electron will transition to a higher energy level corresponding to the energy of the incident photon. The higher the energy level of the incident photon, the larger the transition the electron is capable of making. Given a sufficiently high energy, an electron can leave an atom's orbit. Radiation with sufficient energy to remove an electron from an atom is known as ionizing radiation, as an atom with its electron removed becomes an ion. Only the shortest wavelength 
radiations such as gamma rays, X-rays, and extreme UV rays are capable of ionization. Ionization degrades polymer in two ways: through cascading collisions of emitted electron with other molecules and through free radical production. Together, these two mechanism degrade materials through the division and formation of bonds, altering it from its original state. While lower energy UV radiation is non-ionizing, it does possess similar bond energy to many organic compounds (300 $415 \mathrm{~kJ} / \mathrm{mol})$ [21]. Hence, when polymers are exposed to UV radiation, it can result in bond breakage and free radical formation resulting in microscopic degradation through chain scission noted through discolouration and decrease in mechanical properties. As polymers are often surrounded by an oxygen rich environment, this process is conventionally know as photo-oxidation as the free radical chain segments will often react with ambient oxygen promoting further bond breakage, similar to thermal oxidation. In addition, the energy from UV radiation absorbed by atoms is transferred into kinetic energy, increasing a polymer's temperature. Given sufficient exposure, this process can help promote thermal oxidation as previously described.

A great deal of research has been carried out investigating the capability of UV radiation to degrade polymers and FRPMC. Predominately, studies investigating UV radiation look at the effects of weathering which look at the synergistic contributions of factors which a material may have to face in an outdoor environment. This can include the effects of temperature, moisture, precipitation, wind and UV radiation. Early work in this area performed by Roylance demonstrated weather's degradation capability [123]. Epoxy laminate composites were exposed to natural weathering at various location around the world for a two-year period. Flexural and tensile tests revealed up to a $15 \%$ and $32 \%$ drop in strength, respectively, albeit with a large degree of variability. Shokrieh and Bayat took a more controlled approach, 
examining strictly the effects of UV radiation by exposing polyester resin samples to UV only expose in an ultraviolet chamber [124]. Through accelerated exposure intended to replicate one year of UV exposure outdoors, their work demonstrated a $34.8 \%$ decrease in tensile strength and an $18.8 \%$ decrease in shear strength. Work by Kumar et al. and Nakamura et al. investigated the individual and synergistic effects of UV radiation and condensation on the degradation of an epoxy/carbon composite at elevated temperatures $[32,33]$. It was determined that $500 \mathrm{~h}$ of exposure to a 0.68 $W / m^{2} \mathrm{UV}$ source resulted in minimal degradation, and that sequential exposure to UV radiation and condensation resulted in a linear superposition of change in mass as a result of the two exposures. However, interestingly, when the composite was exposed to an equivalent duration of cyclical exposure to UV radiation and condensation, a continuous weight loss was noted throughout the specimen's exposure. In turn this resulted in a $29 \%$ decreases in transverse tensile strength oppose to the $21 \%$ decrease noted though sequential exposure. This outcome was stipulated to be a result of the removal of the UV modified surface layer through condensation, allowing for the UV radiation to more effectively oxidize sub-surface material. Furthermore, this experiment highlights the importance in studying the synergistic contribution of a multi-factor exposure.

While review has demonstrated that UV radiation has the potential to cause significant degradation to FRPMC through surface oxidation, especially through cyclic, synergistic exposure with moisture, circumstantially, the composite in question will be utilized in an aircraft interior and thus will not have direct exposure to solar radiation. Additionally, where the composite structure is placed internally, it will have minimal expose to through-glass radiation. Lastly, it was identified that there are 
no significant sources of UV radiation within the aircraft. For these reasons, investigation of degradation of FRPMCs due to UV radiation was not pursued further in primary research. However, future research may be warranted for synergistic studies observing the contribution of degradation due to UV radiation on external aircraft components.

\section{A.2.2 Biological}

Biological degradation is degradation of a material resulting from interactions with some form of biological agent. When investigating FRPMC degradation, fungi and bacteria are typically of greatest concern due to their relative prevalence in the working environment of composite materials. However, other biological agents such as plants, algae or shellfish such as barnacles, may also pose potential harm depending on the environment [21]. Biological degradation may occur through either physical or chemical mechanisms such as erosion between the composite and organic matter or corrosion resulting from the release of an acidic or caustic solutions from the fouling organism in an effort to deteriorate the host material for food [125].

Currently, there is limited research describing the impact of biological degradation on the mechanical properties of composite materials. Work by Tascioglu et al. investigated the effects of brown and white rot fungus growth on the structural integrity of a

phenolic/glass composite for civil applications [126]. While the composite specimens were able to sustain growth over a 24 -week period, deterioration to mechanical properties appeared to be relatively minor with the greatest observed detriment being a $7.4 \%$ decrease to interlaminar shear strength resulting from exposure to the brown rot fungus. Similarly, work by Gu et al. investigated the impact of both fungal and 
microbial fouling on composite degradation $[125,127,128]$. From exposure to a range of fungi and bacteria, it was demonstrated, through microscopy, that an epoxy-carbon composite could sustain fungal and bacteria growth and that fungal colonization occurred preferentially on the carbon fibres. However, the researchers noted from the trials that there was minimal statistically significant deterioration to the composites mechanical properties. Wagner et al. performed a similar experiment demonstrating preferential bacteria colonization on surface anomalies and a negligible impact of colonization on epoxy-carbon and epoxy-glass specimens' impact strength [129].

With limited resources for review, further research into biological degradation of FRPMCs is warranted, especially for civil and marine applications for which biological agents are known to be prevalent in the composite's working environment. However, it is important to note that microorganisms preferentially grow in warm and humid climates. Additionally, FRPMCs which are more water resistant tend to present a more hostile environment for biological growth [21]. In the studies reviewed, preferential condition such as direct exposure to bacteria cultures, elevated porosity to help stimulated moisture diffusion, conditioning with growth medium and incubation in soil were implemented in order to stimulate growth. Realistically, the controlled environment of an aircraft's interior would not provide favourable conditions for biological growth. Hence, because of a lack of evidence supporting significant degradation resulting from biological agents and knowledge ff the controlled environment of aircraft interiors, investigation of degradation of FRPMC due to biological agents was not pursued further in primary research. 


\section{A.2.3 Salt Fog}

As the name suggests, salt fog is a fog, a suspension of water droplets in air, which contain dissolved salt ions. For FRPMCs, salt fog presents the same concerns for degradation as exposure to normal water fog or high humidity environments, that is, the potential for surface condensation, moisture ingress and matrix degradation. However, the presence of salt in condensate presents additional reasons for concern including the potential of corrosion due to electrochemical reaction or formation of acidic/alkaline solutions; and the physical and electrical impairment as a result of salt deposition [22]. Because the epoxy-carbon composite in question is primarily a structural member, impedance of motion for moving parts and electrical conductivity are not of concern. However, the potential of corrosion elicits concern over long term structural constancy.

Salt fog is a concern for aircraft operating in a maritime environment and thus has been identified as a factor of interest for investigation in primary study. Salt fog does not present a concern through its presence in air but rather its ability to condense on, be absorbed by and react with the FRPMC. Hence, its effects may be tested in a similar manner to those of water or chemical ingress. Nonetheless, the potential of degradation due to electrochemical reaction does pose a unique problem. The primary concern for composites regarding electrochemical reaction is the potential for degradation as a result of galvanic corrosion. This issue is discussed in Section A.2.4. 


\section{A.2.4 Galvanic Reaction}

When salt fog condenses on a carbon fibre composite which is in contact with a metal, it forms the electrolyte of an electrochemical cell. Because of the dissimilar standard electric potential between the metal and the carbon fibre, an electrochemical reaction is driven forwards in an effort to achieve equilibrium. This reaction proceeds through the following mechanisms:

$$
\begin{aligned}
& M_{1(s)} \rightarrow M_{1(a q)}^{y}++y e^{-}(\text {anode }) \\
& M_{2(a q)}^{y}+y e^{-} \rightarrow M_{2(s)}(\text { cathode })
\end{aligned}
$$

Where $M_{1}$ and $M_{2}$ represent two conductive materials of different electrical potential. In this system, there are two half-cell reactions which take place. The anodic reaction involves the solid anode's dissociation into ions and their associated electrons. The ions, which are released in the electrolytic solution are then free to migrate to the cathode where the cathodic reaction may then take place. This involves an aqueous ion's recombination with electron and resulting deposition on the cathode surface. In the case of a carbon fibre composite in contact with a metal (such as a screw, bolt, rivet, etc.) the metal, which characteristically has a more negative standard electrode potential, will form the anode and the carbon, which has a more positive standard electrode potential, will be the cathode. Hence, galvanic corrosion will cause the metallic anode to degrade over time. Furthermore, research has demonstrated the potential of this reaction to degrade FRPMC composites. Tucker and Brown have demonstrated that this ion formation and deposition can result in a concentration gradient which prompts diffusion and blister formation through osmotic pressure, 
a phenomena not noted in a system of otherwise identical conditions, which was not subjected to galvanic coupling [130]. Subsequent studies on galvanic corrosion of metal-carbon fibre systems have shown similar degradation, but have demonstrate that application of a non-conductive polymer or fabric layer between metal and carbon fibre can significant decrease galvanic corrosion effects [131,132].

To avoid the effects of galvanic corrosion, the FRPMC structure in question will have a fibre lay-up which uses one or more layers of glass fibre on its outer surfaces. As an insulator, glass inhibits the flow of electrons. This prevents a conductive pathway between the metal of multi-material assemblies fastened to the composite structure and the composite's embedded carbon fibre preventing the formation of a galvanic cell, thus preventing galvanic corrosion. Hence, while literature has revealed the potentially detrimental effects of galvanic corrosion on carbon fibre composites, based on the preventative measure implemented, investigation of the degradation of the FRPMC due to galvanic corrosion was not pursued further in primary research. 


\section{Appendix B}

\section{Standard Operating Procedures}

The following appendix details SOPs followed over the course of experimentation.

SOPs were implemented to ensure safety and consistency, and may allow for study validation through test replication if required in future work. 


\section{B.1 Single-Sided Flexural Sample Fluid Uptake Metrology: Standard Operating Procedure}

\section{Location}

Thermo-Lab, ME 2250

Carleton University

1125 Colonel By Drive

Ottawa, ON K1S 5B6

\section{Equipment}

$\begin{array}{cc}\begin{array}{c}\text { Quincy Labs Laboratory Oven } \\ (2 \mathrm{x}) \text { Sample Arrays }\end{array} & \text { Nitrile Gloves } \\ \text { Snap-On Torque Screwdriver } & \text { Paper Towel } \\ \text { Santorius Microbalance } & \text { Weigh Paper } \\ \text { Hex Key } & \text { Safety Glasses } \\ \text { Transfer Pipettes } & \text { 4.5-qt Pyrex Dish } \\ 4 \text { x 50mL beaker } & \text { Oven Rack } \\ \text { Kapton Tape } & \text { Scissors }\end{array}$

\section{B.1.1 Preface}

This document details the standard operating procedure (SOP) for measuring the mass change as a result of fluid uptake of carbon-fibre reinforced epoxy samples subjected to single surface fluid exposure at elevated temperatures.

\section{B.1.2 Procedure}

Note: Always wear safety glasses and nitrile gloves when removing arrays from or adding arrays to the conditioning oven, transferring fluid to or from the sample wells and when handling the carbon-fibre samples. Wear heat resistant gloves when removing arrays from or adding arrays to the conditioning oven.

Note: Ideally, this process should be completed as fast as possible in an effort to reduce the time for which samples are not being held at conditioning temperature. 
However, safety, accuracy and consistency should always take precedence over performance speed.

\section{Array Removal from Oven}

1. Place one of the available oven racks on top of a 4.5-qt Pyrex in front of the conditioning oven

2. With the fumehood sash fully open, open the conditioning oven's door and, using two hands, carefully remove one of arrays of interest and set it onto of the oven rack. Close the door immediately following the array's removal.

3. Record the time the array was removed.

4. Visually inspect the array; not any dramatic changes to fluid level (i.e. if it is low or absent from any of the wells) if there are any changes to how the thermocouples are secured, evident cross-contamination of fluids or any other discrepancies from the array's original appearance. Take pictures if applicable.

5. Remove any Kapton tape used to seal the cells or hold thermocouples in place

6. Using clean transfer pipettes, transfer the fluids from the sample wells into their designated beakers; ocean water will be transferred directly into the organic waster bottle. In order to remove the last bit of fluid, tip the array forward slightly and removing and remaining liquid which pools

7. Using individual paper towels, remove the bulk of any remaining fluid by inserting the paper towel into the cell and performing a single wipe of the sample's surface

\section{Sample Removal from Array}

1. Transfer the Pyrex dish, oven rack and array to the bench adjacent the microbalance

2. Flip the array over; using the torque screwdriver, loosen each of the stainless steel hex nuts

3. Remove the hex nuts, washers and cap-head screws by hand Note: Due to limited clearance, some of the cap-head screws may be difficult to remove from the array. Apply moderate force with thumbs. If excessive force is required, unscrew the screws from the array using the hex key 
4. Remove the top plate from the array. Visually inspect and record any discrepancies to the interior surfaces of the top and bottom plates and gasket material.

5. Remove the samples from the array. Place samples exposed to the same fluid together in sample bags and seal the bags until sample temperatures are below 30C.

6. Clean off the components of the sample array using DI water and paper towel.

\section{Sample Metrology}

1. Place a weighing paper on the balance, close the balance's enclosure and TARE balance

Note: If significant fluctuations are noted in the balances readout after the balance has been tared, this maybe due to static attraction between the balance's metal surface and the waxy weigh paper. If this occurs, crease the weigh paper and place it concave side up on the balance. Re-tare and inspect the readout to ensure minimal fluctuations

2. Focusing on one sample set at a time, remove a sample of interest from the sample bag

3. Wipe the specimen five times lengthwise using a lint-free paper towel making sure to use a new dry section of paper towel for each repetition; gently dab following five wipes

4. Repeat step three on the top and bottom faces of the specimen

5. Wipe the through-thickness surfaces a single time

6. Place the sample on the weigh paper, close the balance's enclosure and allow the reading to stabilize. Record the reading as soon as the balance stabilizes.

7. Remove the sample from the balance; measure and record length, width and thickness values using the average of three measurements

8. Place it back in its sample bag. Repeat steps 1 through 7 for each sample making sure to seal each bag after completion of a sample set, using a new weighing paper after each sample set and re-taring after each sample

\section{Reassembling Sample Arrays}

1. Remove the samples from their bags and place them back into the seats in the bottom portion of the sample array. Samples must be oriented bag side up, 
with the ID on the tool side face in the direction which faces the front of the oven

2. Place the gasket atop the samples, aligning sample holes and screw holes. This may require manipulating the gasket locally around the sample holes

3. Place the top plate on top the gasket making sure all holes are still in good alignment

4. Ensure both the plates and gasket are in proper alignment, as indicated by the numerical indicator on the front right corn of the array

5. Re-insert each of the cap-head screws; flip the array over, place a washer on a screw and screw the nut on the screw until it each is hand-tight

6. Using the torque screwdriver and the hex key, staring at one of the arrays corners, torque the screws to 10 inlbs make sure to follow a raster pattern. Apply this torque in the same pattern to ensure an even loading distribution has been achieved.

\section{Returning Array to Oven}

1. Place the array back on top of the oven rack, well side up, in front of the oven

2. Using the same transfer pipettes, transfer the fluid removed from the array back into their respective wells. After fluid reintroduction, each well should be approximately half full of fluid. If there is not enough fluid (or in the case of the ocean water sample), supplement with fresh fluids.

Note: If at any point during this process there is concern that a sample well may have been cross-contaminated, disposed of the well's fluids in the organic waste container, wipe out any remaining fluid with a paper towel and reintroduce fresh fluid into the well

3. Seal all cells which contain volatile fluids (DI water, substitute ocean water and ethylene glycol) with Kapton tape

4. Re-adhere the thermocouples to the array in the same orientation they were in prior to the arrays disassembly

5. Inspect the arrays for any visual signs of leaking

6. Open the oven and place the completed array back on its respective shelf

7. Record the time the array was reintroduced to the oven 
8. Repeat this process for each of the arrays of interest

9. Once all required sample mass have been taken, shuffle the arrays such that each array is one shelf above its previous location and the array on the top shelf is moved to the bottom shelf. Record the new array orientation and close the oven door. 


\section{B.2 Short Beam Shear Fluid Uptake Metrology: Standard Operating Procedure}

\section{Location}

Thermo-Lab, ME 2250

Carleton University

1125 Colonel By Drive

Ottawa, ON K1S 5B6

\section{Equipment}

$$
\begin{array}{cc}
\text { Quincy Labs Laboratory Oven } & \text { Heat Resistant Gloves } \\
\text { Sample Test Tubes }(18 \mathrm{x}) & \text { Nitrile Gloves } \\
\text { SBS Samples }(18 \mathrm{x}) & \text { Paper Towel } \\
\text { \#0 Test Tube Stoppers (x18) } & \text { Weigh Paper } \\
\text { Test Tube Rack } & \text { Safety Glasses } \\
\text { Transfer Pipettes } & \text { Digital Calipers }
\end{array}
$$

Sartorius Microbalance Equipment

\section{B.2.1 Preface}

This document details the standard operating procedure (SOP) for measuring the mass change and changes to dimensions, resulting from fluid uptake, of carbon-fibre reinforced epoxy SBS samples subjected to fluid immersion at elevated temperatures.

\section{B.2.2 Procedure}

Note: Always wear safety glasses and nitrile gloves when removing or adding test tubes to the conditioning oven, transferring fluid to or from the test tubes and when handling carbon-fibre samples. Wear heat resistant gloves when removing test tubes from the conditioning oven.

Note: Ideally, this process should be completed as fast as possible to reduce the time for which samples are not being held at conditioning temperature. However, safety, accuracy and consistency should always take precedence over performance speed. 


\section{Rack Removal from Oven}

1. Remove the test tube rack of interest from the conditioning oven and place it in the fumehood

2. Allow test tubes to cool at room temperature for 20 minutes; this allows them to cool to approximately $30 \mathrm{C}$

3. Record the time/date the test tubes were removed from the conditioning oven

4. Transfer the test tube rack to the bench next to the microbalance

\section{Sample Metrology}

1. Make sure the microbalance is level by inspecting the spirit level to ensure the bubble is in the center of the circle. If not centered, adjust the height of the balance's front legs using their respective adjustment knobs

2. Turn on the microbalance

3. Place a weighing paper on the balance, close the balance's enclosure and TARE balance

Note: If significant fluctuations are noted in the balances readout after the balance has been tared, this may be due to static attraction between the balance's metal surface and the waxy weigh paper. If this occurs, crease the weigh paper and place it concave side up on the balance. Re-tare and inspect the readout to ensure minimal fluctuations

4. Focusing on one sample set at a time, remove a sample of interest from its test tube by first pouring its conditioning fluid into a test tube containing the same conditioning fluid (Refer to Figure 1) and then pouring the sample (and any residual fluid) on to a paper towel

5. Wipe each of the specimen six surfaces, five times lengthwise using a lint-free paper towel making sure to use a new dry section of paper towel for each repetition

6. Place the sample on the weigh paper, close the balance's enclosure and allow the reading to stabilize. Record the reading as soon as the balance stabilizes.

7. Remove the sample from the balance; measure and record length, width and thickness values using the average of three measurements

8. Place the sample back in its respective test tube; re-immerse the sample in the previously transferred conditioning fluid. 
9. Repeat steps 4 through 8 for each sample making sure to use a new paper towel for each sample and re-taring the balance as required

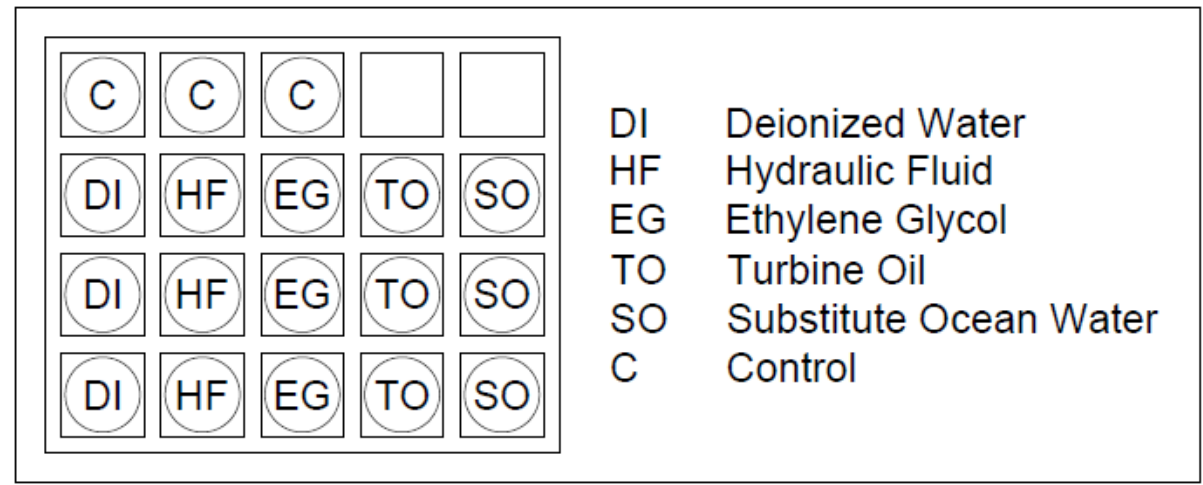

Figure B.1: Test tube rack conditioning fluid layout.

\section{Returning Rack to Oven}

1. Ensure all test tube stoppers are set gently in the top of the test tubes. Stoppers which have been pushed into the test tubes may prevent volatiles from escaping causing a build up of pressure and the stopper to pop-out of the test tube after it has been returned to the conditioning oven.

2. Inspect each of the test tubes to make sure all samples are immersed in their respective fluids. If a sample is not immersed add more fluid.

3. Open the oven door and place the test tube centrally on the oven rack; firmly close the oven door

4. Record the time the test tubes were returned to the conditioning oven 


\title{
B.3 $25 \mathrm{kN}$ MTS 810 Test Frame Flexural Testing: Standard Operating Procedure
}

Location

Structures Laboratory, ME 2260

Carleton University

1125 Colonel By Drive

Ottawa, ON K1S 5B6

\section{Equipment}

\author{
MTS 810 Material Testing System Flextest SE Controller \\ 3 or 4-Point Flexural Test Fixture 634.12 Axial Extensometer \\ Personal Computer and Monitor Flexural Samples
}

\section{B.3.1 Preface}

This document details the SOP for operating the MTS 810 Material Testing System, with specific focus on flexural testing. While many of procedural steps will be the same for all forms of material testing using the MTS 810 Material Testing System, the operator MUST have had preliminary training on the system, during which the material testing procedure of interest (ie. tension, compression, fatigue, etc.) was followed and have been given permission from the lab technologist before operating the material testing system independently. For information on testing requirement, procedures, or preliminary training, contact the lab technologist.

\section{B.3.2 Procedure}

Note: Always wear safety glasses and closed-toe shoes while working in the vicinity of the material testing systems

\section{Hydraulics Activation}

1. Log in to the first computer terminal (the farthest on to the right) using the account name and password provided below the monitor 
2. Open the material testing system's GUI by double clicking on the "Station Manager" icon located on the Desktop

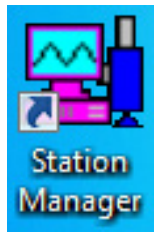

3. In the "Open Station" window, select the "MTS.cfg" configuration file and select the "Basic Settings" parameter set from the provided list; click "Open"

4. In the "Station Manager" window, under the "Station Controls" header, check the "Exclusive Control" check box; this will allow the computer to take control of the adjacent Flextest SE controller

5. Click the icon with two lines in line with the HPU label. Wait a few seconds for the audible clicking noises to stop and then click the adjacent icon with three lines; this initiates the hydraulic system by stepping-up the hydraulic power unit (HPU)

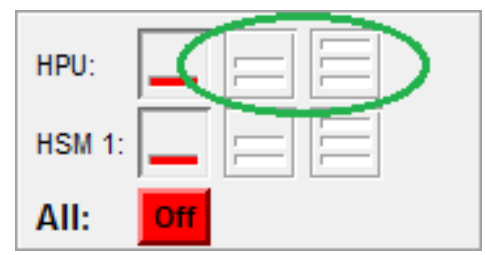

\section{Test Fixture Installation}

6. Standing in front of the material testing machine, unlock the crosshead by turning the right-most switch to face the symbol which looks like an opening collet 


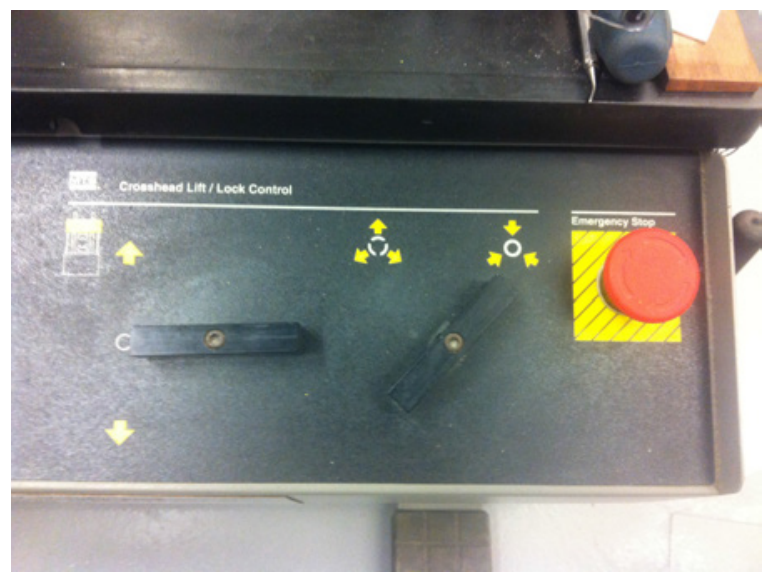

7. Raise or lower the crosshead, using the adjacent crosshead lift switch such that once the test fixtures has been put in place there is at least six inches of separation between the upper and lower test fixtures

8. Lock the crosshead by turning the right-most switch to face the symbol which looks like a closed collet

9. Open the top and bottom grips using the hydraulic grip control; arrows out to release, arrows in to grip

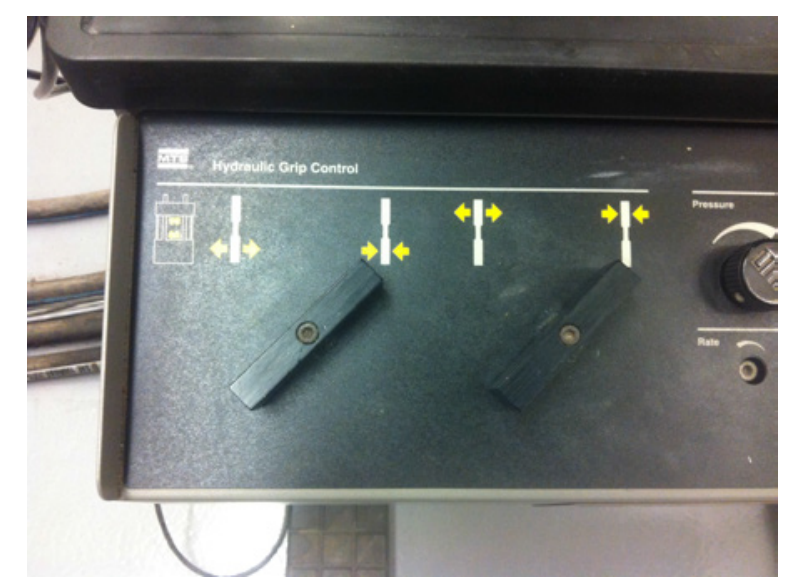

Note: If there is already a test fixture in place, grasp the test fixtures with one hand while releasing the grip with the other such that the fixtures will not fall out once they are released from the grip

10. Increase the hydraulic grip pressure to maximum

11. Place the bottom fixture in the hydraulic grip such the grip mount sits flush with the bottom, internal face of the grip 
12. Close the bottom grip; make sure that it firmly grasps the test fixture grip mount

13. Repeat this process for the top fixture

\section{System Setup}

14. Log in to the third terminal (the farthest one to the left) with the "admin" login using "mts\#admin1" as the password

15. Open the material testing system's GUI by double clicking on the "Station Manager" icon located on the Desktop

16. In the "Open Station" window, select the "ftse.cfg" configuration file and select the parameter set labelled with your name from the provided list; click "Open" Note: This SOP assumes the user will have generated a parameter set previously during preliminary system training

17. If the "Function Generator" sub-window opens up in the "Station Manager" window, click "Basic TestWare" under the "Applications" drop-down menu

18. Close the "Test Setup" window which pops up

19. Under the "Station Controls" header, Open the "Detectors" window by selecting the icon which depicts the upper and lower limits of a sinusoidal wave

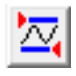

20. In the "Detectors" window which opens, set the test specific upper and lower limits for stroke and load. Then, select the "Station Power Off" action for each limit which the system will perform if any of the specified limits are reached; 
this is a very important step as employing system limits will (if done properly) prevent the system from moving out off, or applying excessive loads in the testing volume, thus preventing damage to system components or external equip. Make sure that you have a good understanding of your testing limits

Note: Whenever entering values into the material testing system software, ALWAYS press "Enter" to ensure the value is accepted by, and saved to the system

Note: The material testing system software operates under the convention that loads and displacements operating in tension are positive $(+)$ and that loads and displacements operating in compression are negative (-)

21. Close the "Detectors" window

22. Check to see if there is a line illuminated red under the "Station Limits" heading; if there is, this is and indication that either there is an interlock error or the system is currently outside of the previously defined detector limits

23. Below the "Station Limits" indicator, click the Interlock 1 "Reset" button. If a line is still illuminated red, this indicates that the system is outside of the detector limits. Click the Program 1 "Reset/Override" button such that timer which appears below the button indicates 60 , signifying the system will be in override for 60 seconds

Note: While the system is in override it will not adhere to the predefined detector limits. Make sure that any operation performed while in override will not result in damage to the system or pose a safety risk for those in the surrounding area

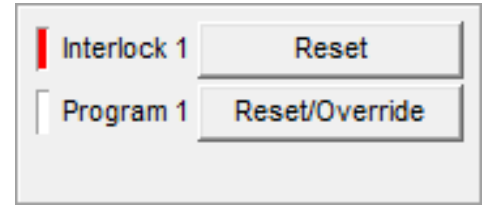

24. As was done during the initial hydraulic activation, step up the HPU

25. In a similar fashion, step up the hydraulic servo motor (HSM) by selecting the icon with two lines next the HSM 1 label, waiting a few seconds and then selecting the adjacent icon with three lines 
26. Under the "Station Controls" header, Open the "Manual Controls" window by selecting the icon which depicts arrows above and below a line with a slider

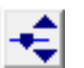

27. In the "Manual Controls" window, click the "Enable Manual Command" check box

28. Ensure the Channel is set to "Ch 1" and the Control Mode is set to "Stroke"

29. Using the manual control window, move the lower piston such that the lower fixture is located within the predefined detector limits. The manual control window provides four means of manually controlling the lower piston; manually entering a position in the "Manual Cmd" field relative to the piston's tared "zero" position; clicking and dragging the slider below the "Manual Cmd" line; clicking on the scale region to the right and left of the slider (which increments/decrements the piston $0.1 \mathrm{~mm}$ per click); and clicking the arrows on the end of the sliders scale (which increments/decrements the piston $0.01 \mathrm{~mm}$ per click).

Note: Manual control of the piston by clicking and dragging the slider is illadvised as this can result in rapid and uncontrolled movement of the piston. Pay close attention to sign convention while performing manual operations and never hold down the mouse button on the slider scale

30. Close the "Manual Controls" window

Note: If during this operation, time runs out on the "Reset/Override" timer before getting the lower fixture within the detector limits, repeat steps 1522 , making sure to put more time on the "Reset/Override" timer during step 15 Note: Make sure that once the piston is within the detector limits it is extended at least three inches from the base of the machine 


\section{Aligning the Test Fixtures}

31. Under the "Station Controls" header, Open the "Signal Auto Offset" window by selecting the icon which depicts a line running tangent to a circle

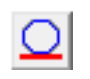

32. Tare the stroke, load and strain "Current Values" by click the adjacent icons which depicts a line running tangent to a circle

33. Close the "Signal Auto Offset" window

34. Re-open the "Manual Controls" window

35. Standing in front of the material testing machine, Unlock the crosshead by turning the right-most switch to face the symbol which looks like an opening collet

36. Lower the crosshead such that extensometer mount on the upper and lower test fixtures is separated by approximately $1 \mathrm{~cm}$; the downward motion of the crosshead is gravity fed so control the speed of the crosshead by switch the crosshead lift switch between neutral and down

37. Lock the crosshead by turning the right-most switch to face the symbol which looks like an closed collet

38. Rotate the bottom test fixture to align the male and female connectors on the extensometer mount

39. Place a sample on the lower test fixture 
40. In small increments, raise the piston using "Manual Controls" window until the components of the extensometer mount are interlocking; for each increment, inspect the test fixtures to make sure the male and female connectors on the extensometer mount are properly aligned

41. Continue to raise the lower test fixture until the rollers on the upper test fixture are immediately above the sample

42. Close the "Manual Controls" window

43. Re-tare the stroke, load and strain in the "Signal Auto Offset" window

44. Close the "Signal Auto Offset" window

\section{Test Setup}

45. Step down the HSM by selecting the icon with two lines next the HSM 1 label, waiting a few seconds and then selecting the adjacent icon with one line

46. Click the open parameter set icon in the outer tool ribbon of the "Station Manager" window

47. Find and select the parameter set labelled with your name; click "Open"

48. In the "Test Setup" window which pops up, click on the "Command" tab

49. Select "Monotonic" as the type, "Ch 1" as the channel, "Stroke" as the control mode, "Absolute" as end level type

50. Input the test specific absolute end level and rate (ie. stroke rate)

51. Under the "Data Acquisition" tab, select "Timed" as the type; select "Strain" from the signals available and add it signals included by clicking the arrow pointing to the right between the signal lists; and select the test specific time between points (the time interval between recorded data points)

52. Under the "Data File" tab, select "User Specified" as the destination file; browse and select a (or enter in a new) file name (this is where the test setup file will be saved); entering in a data header with pertinent information to your test (ie. 
test type, sample geometry, etc.); select "Append" as the data file mode (this will prevent data from being overwritten by simply adding new data to the end of a file if the file name selected for a test which has already been used); and select "Excel as the data file format

53. Under the "Home" tab, select "Ch 1" as the channel, "Stroke" as the control mode, "0.0000" as the absolute end level and "5.0000" as the time; this specifies where the piston will return to and how quickly it will travel there when told to return home

54. Close the "Test Setup" window NOTE: While test parameters can be altered using the "Basic TestWare" window, only adjust test parameters in the "Test Setup" window to ensure an accurate test procedure is saved with the test file

\section{GUI Setup}

55. Looking at the GUI, make sure the "Station Manager" window, "Scope 1" window and "Meters 1" window are oriented relative to each other as shown in the image below; this will aid in troubleshooting any issues with the lab technologist

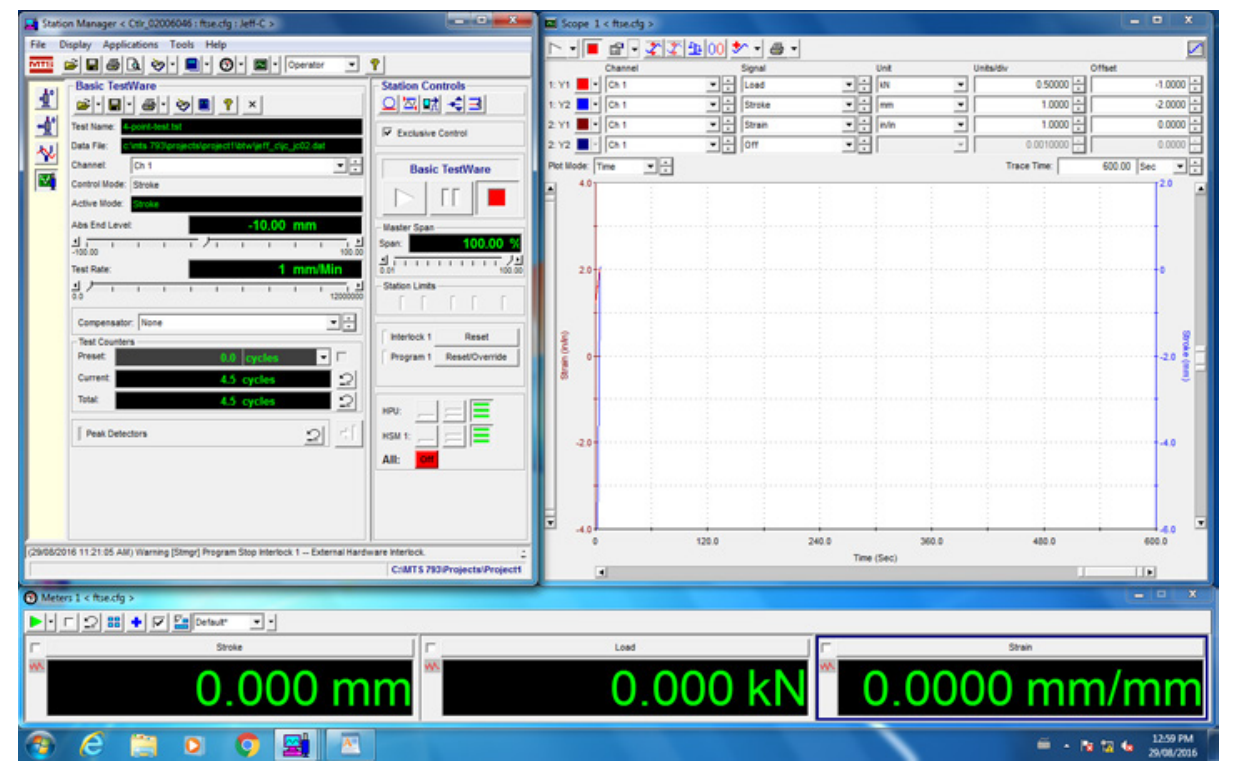

56. In the "Meters 1" window, click the plus icon to add an additional real time parameter; select Strain. The relative position of the parameter meters in the "Meters 1" window can be adjusted by clicking the array icon and adjusting the windows dimensions

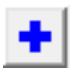


57. Click the small drop down menu next to the play button in the "Meters 1" window and select "Run for Real Time"; this allows all meters to display real time values

58. In the "Scope 1" window, click the small drop down menu next to the play button and select "Program Control"; this allows the graph to display real time values only when a test program is running

59. Click both the "Save Test" and "Save Parameter" icons in the upper left corner of the "Station Manager" window

\section{Running Tests}

60. Clip the axial extensometer to the axial extensometer mount on the top and bottom test fixtures; the extensometer will be connected to power by the lab technologist

61. Make sure the testing area is clear of any other unnecessary equipment which may impede the system's motion

62. Place the plastics shield around the test setup; ALWAYS ensure the shielding is up when actively testing

63. Alert anyone in the surrounding area that you are going to run a test; if someone is working at the adjacent testing setup, position the alternate plastic shield on the other testing system to protect them from debris which may escape from the open side of the primary shield

64. In the "Station Manager" window, under the "Station Controls" heading, click the large play button 
65. If, during testing, something must be inspected or adjusted, press the adjacent pause button to pause the test; press play again to continue the current test

66. Press the adjacent stop button to stop the test; if the stop button is not pressed the test will be stopped when the system reaches one of the detector limits

67. Monitor the test in the "Scope 1" and "Meter 1" windows

68. After the test is complete, remove the axial extensometer from the test fixture; remember to re-insert the extensometer's pin

69. In the "Basic TestWare" window, click the "Return Home" icon which depicts an arrow pointing to a line

70. Remove the sample from the test fixture

71. Repeat steps 3849 to perform multiple, identical tests; remember that because "Append" was selected during the test setup, all data will be saved in the same file unless the data file name is changed

\section{Retrieving Data}

72. Insert a USB drive into one of the slots on the front of the PC

73. Navigate to the file directory where the test files where saved (refer back to the test setup)

74. COPY the files of interest from the file directory to the inserted USB drive

\section{Shutdown}

75. Make sure that a test is not actively running

76. Click both the "Save Test" and "Save Parameter" icons in the upper left corner of the "Station Manager" window 
77. Step down the HSM by selecting the icon with two lines next the HSM 1 label, waiting a few seconds, and then selecting the adjacent icon with one line

78. Step down the HPU by selecting the icon with two lines next the HPU label, waiting a few seconds, and then selecting the adjacent icon with one line

79. Close the "Station Manager" window

80. At the first computer terminal, make sure that a test is not actively running

81. Step down the HPU by selecting the icon with two lines next the HPU label, waiting a few seconds, and then selecting the adjacent icon with one line

82. Close the "Station Manager" window

83. Log off both terminals 


\section{Appendix C}

\section{Orthographic Drawings}

Presented in this appendix are orthographic projection drawings illustrating machined components of the flexural test fixture and conditioning arrays. Components were drafted and modeled in AutoCAD design software. Subsequently, tool-paths were devised in Mastercam CAD/CAM software, prior to milling. 


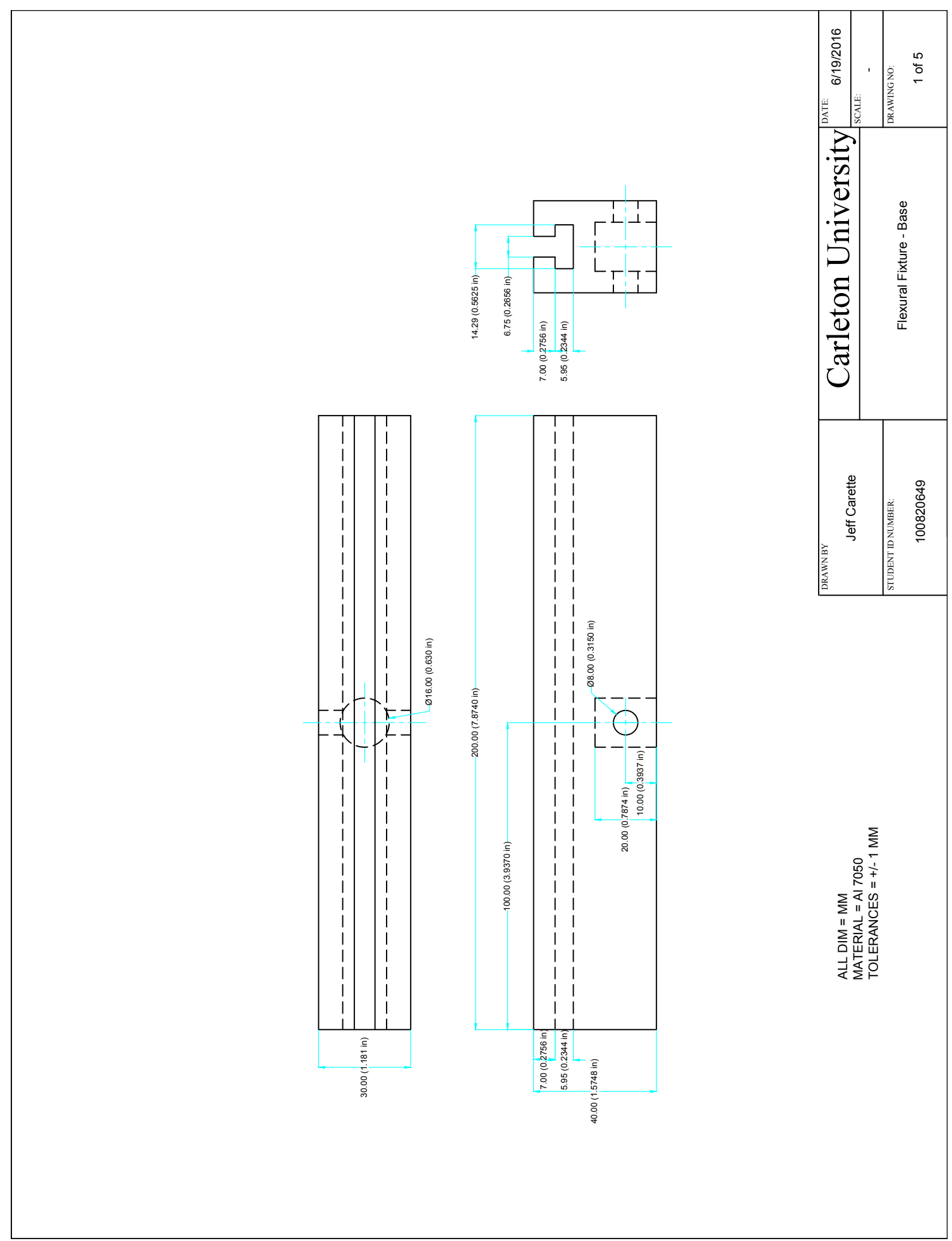




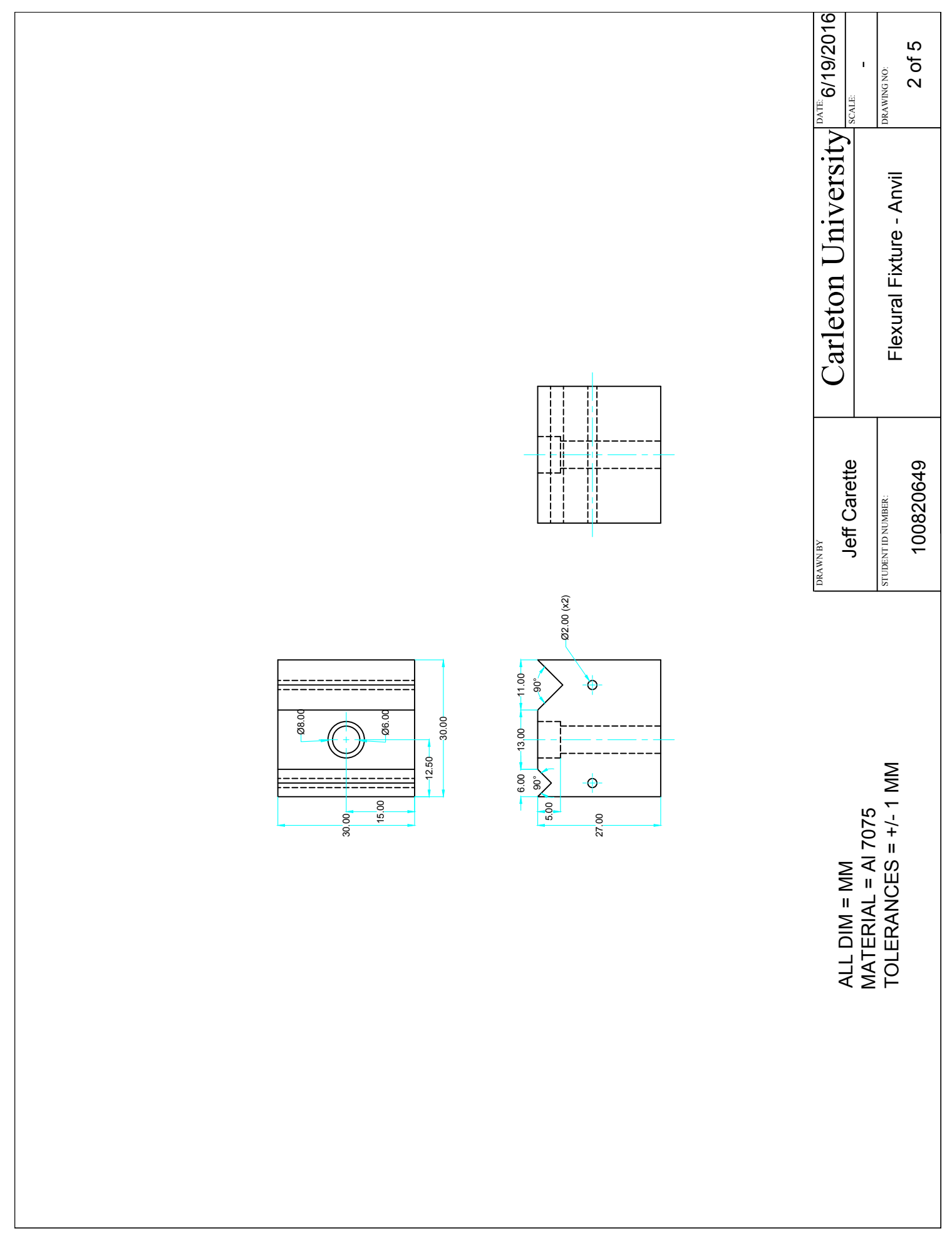




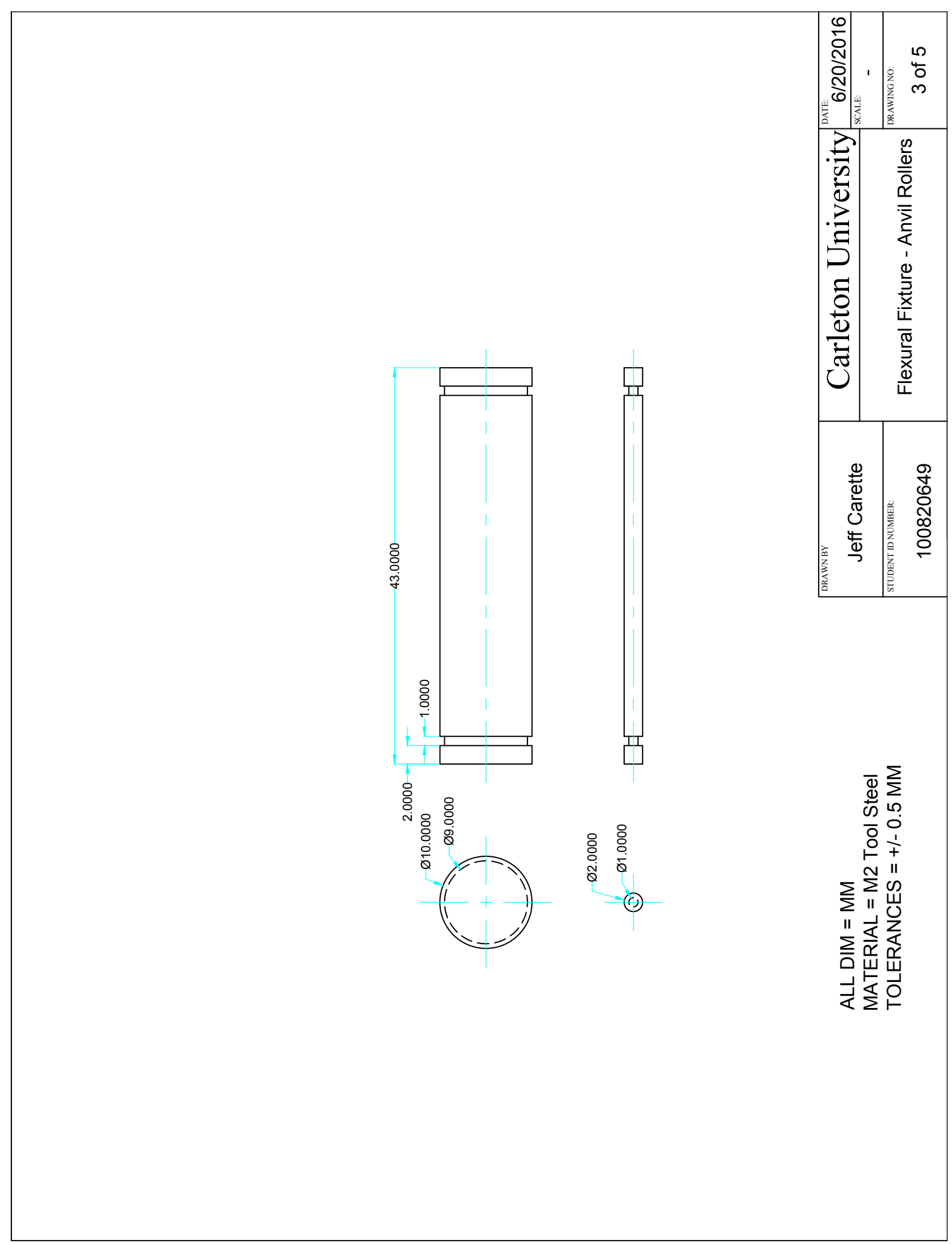




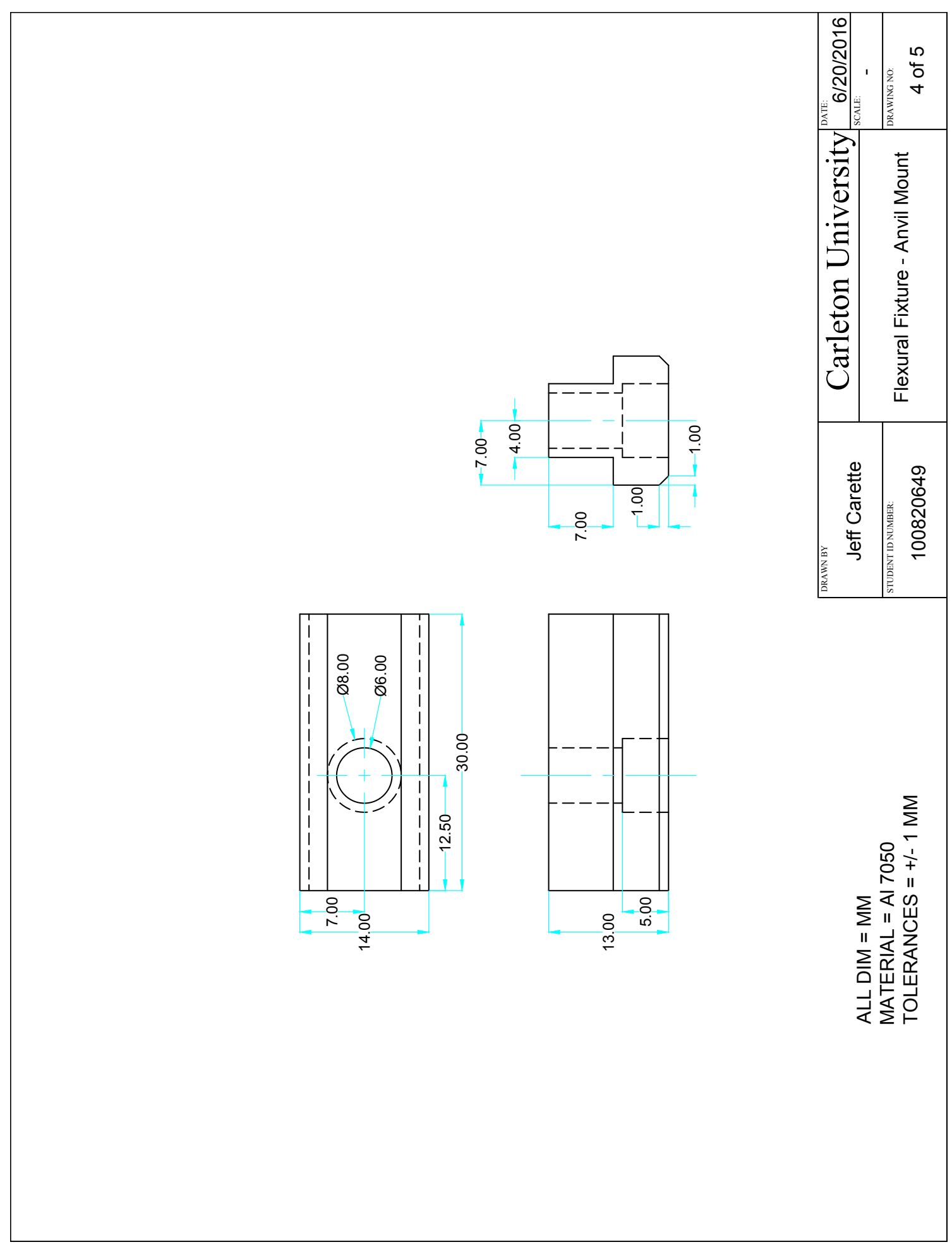




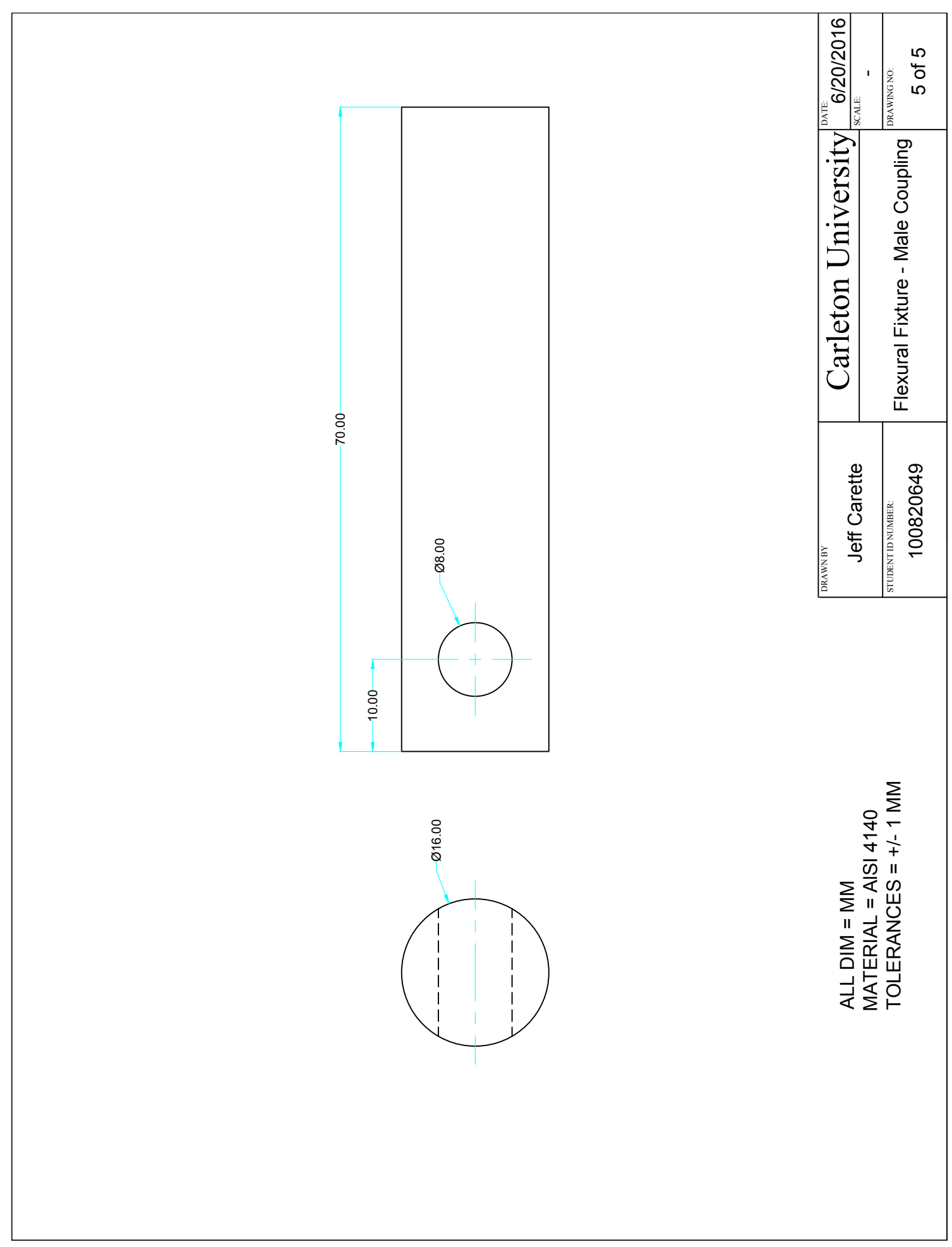




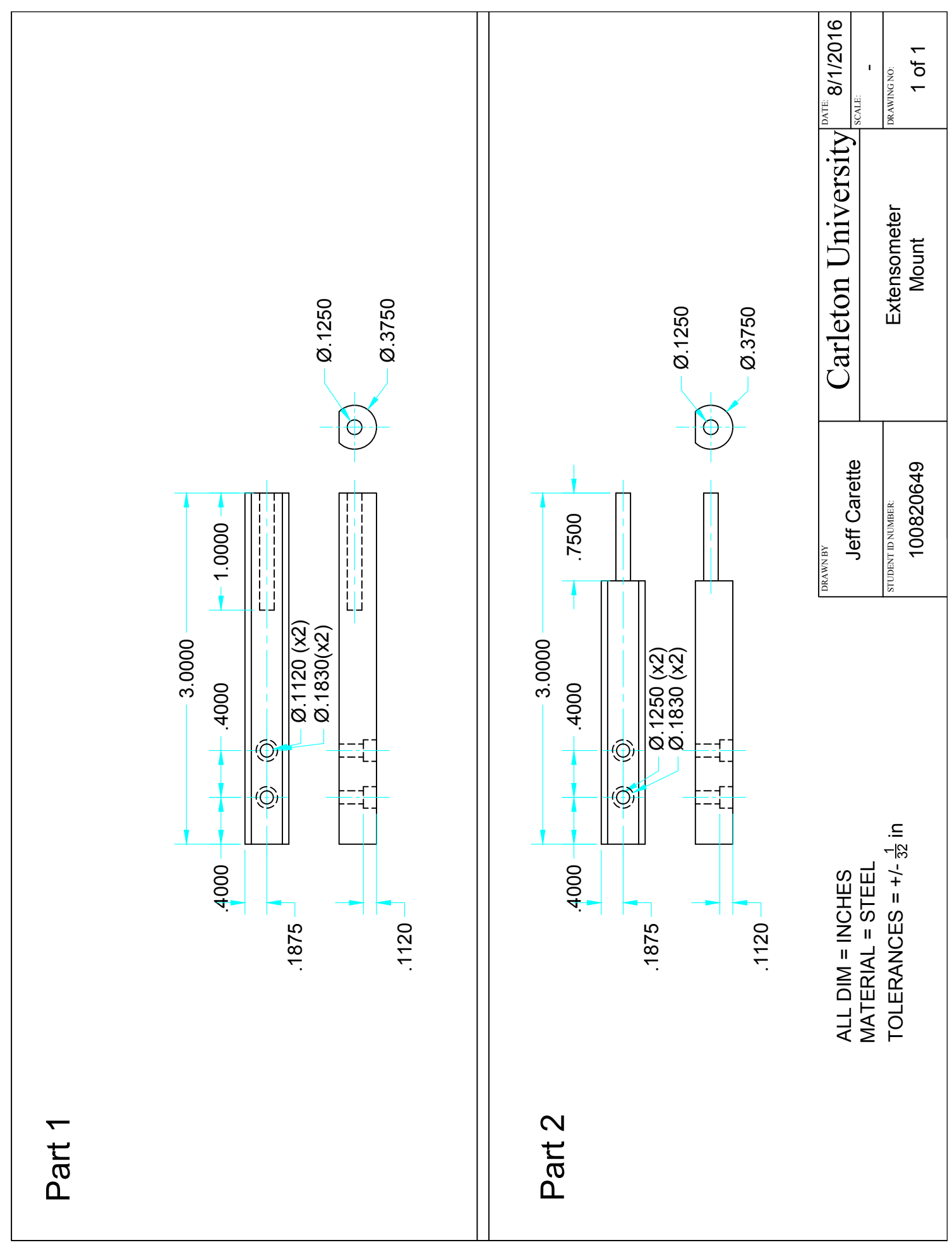




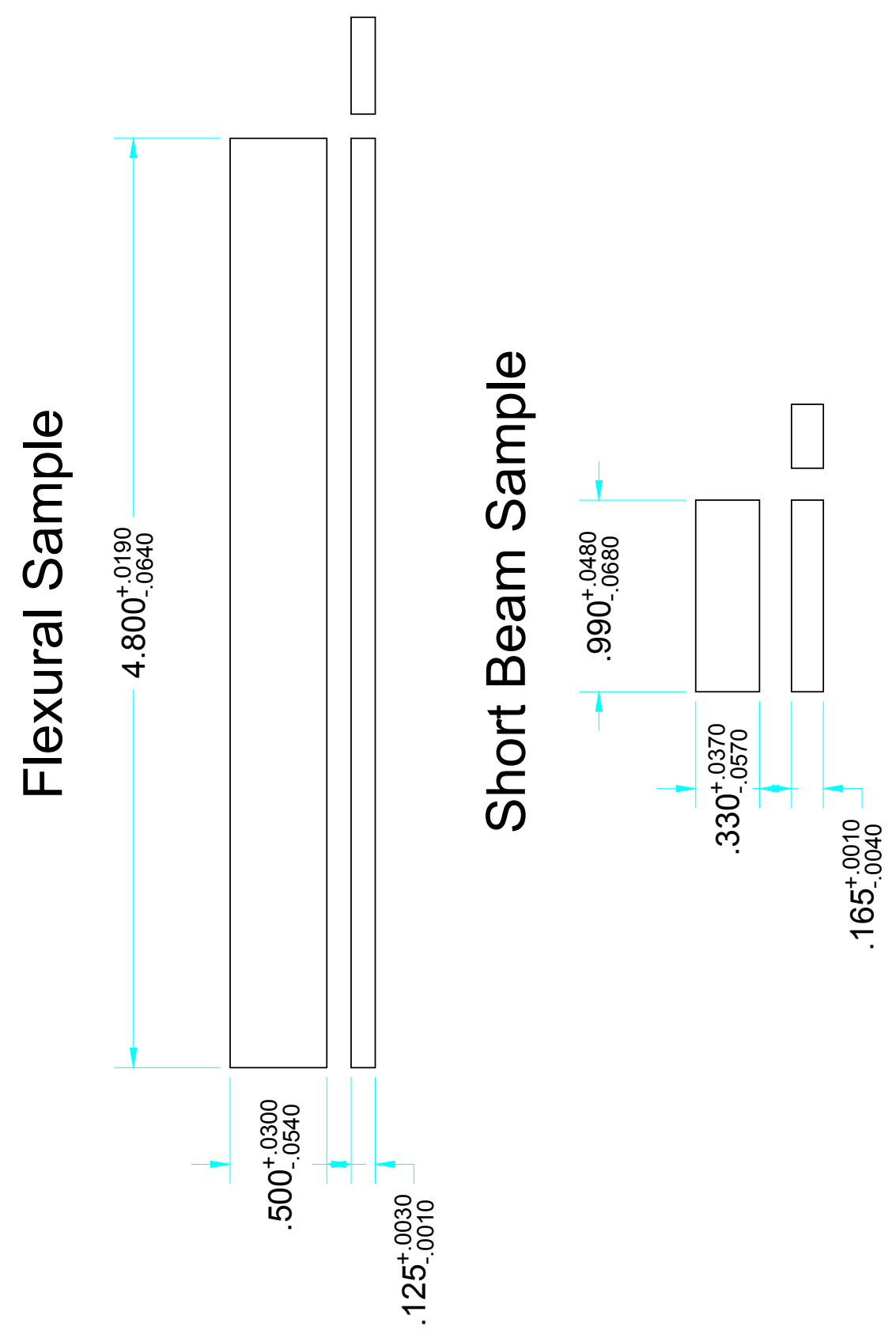




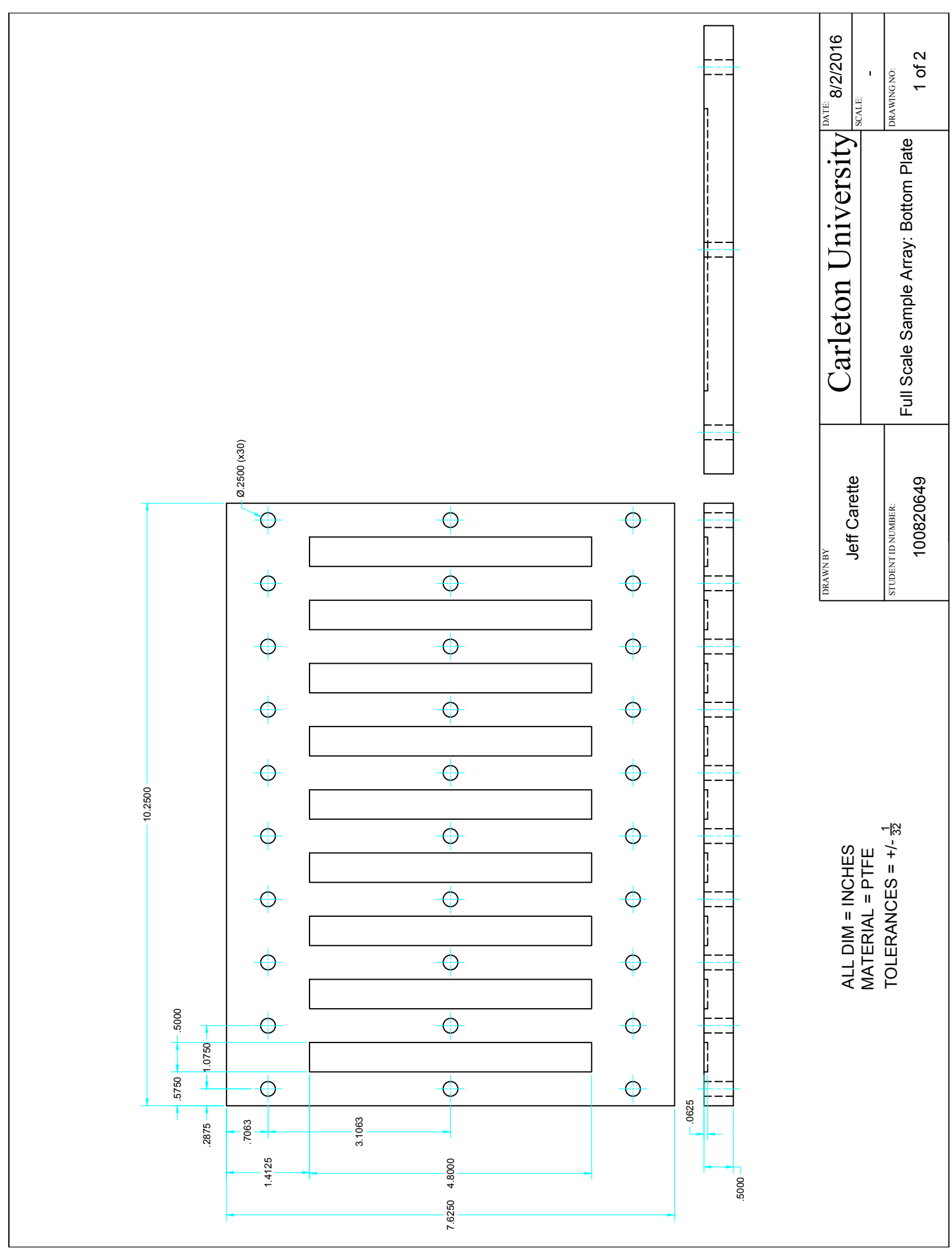



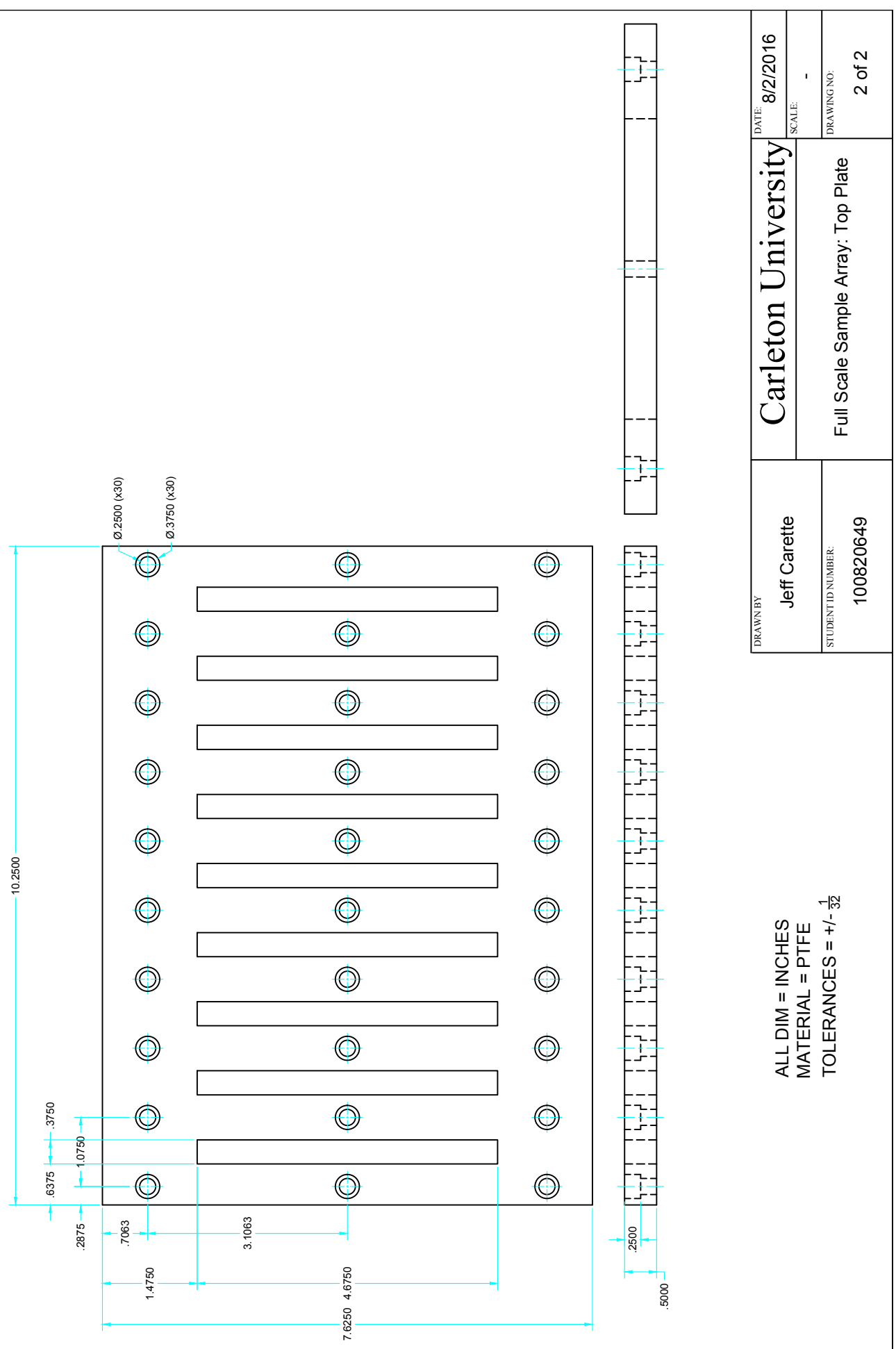

-

=-1-

=-

- -

का

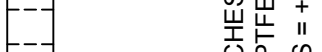

年

政

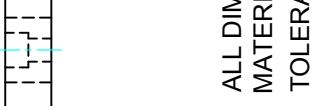

$=-1$

$=-1$

促-

总- 


\section{Appendix D}

\section{Supplementary Results}

Presented in this appendix are experimental results, supplementary to those presented in the Sections 2 and 3 of the thesis body.

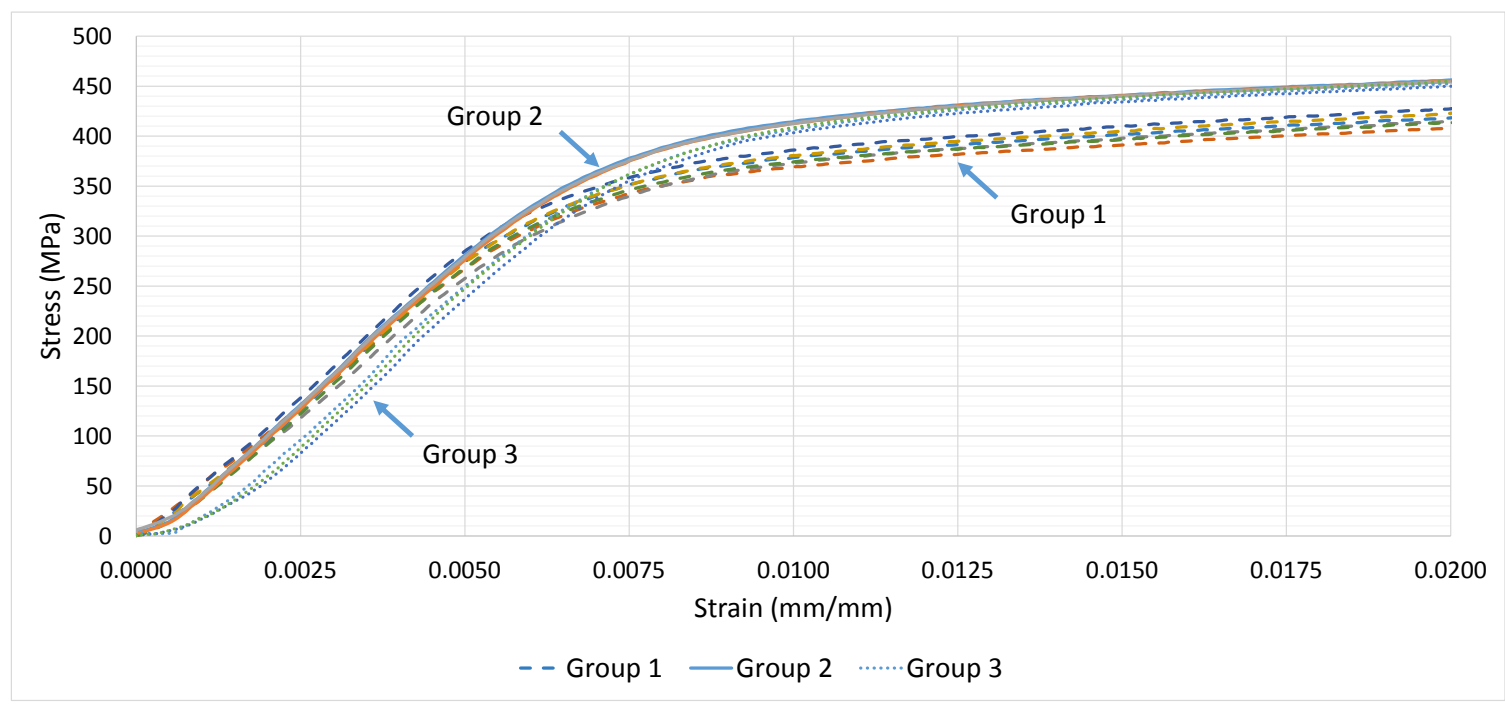

Figure D.1: Stress-strain relationship for 6061-T6, T651 aluminum flexural test; No toe compensation. 

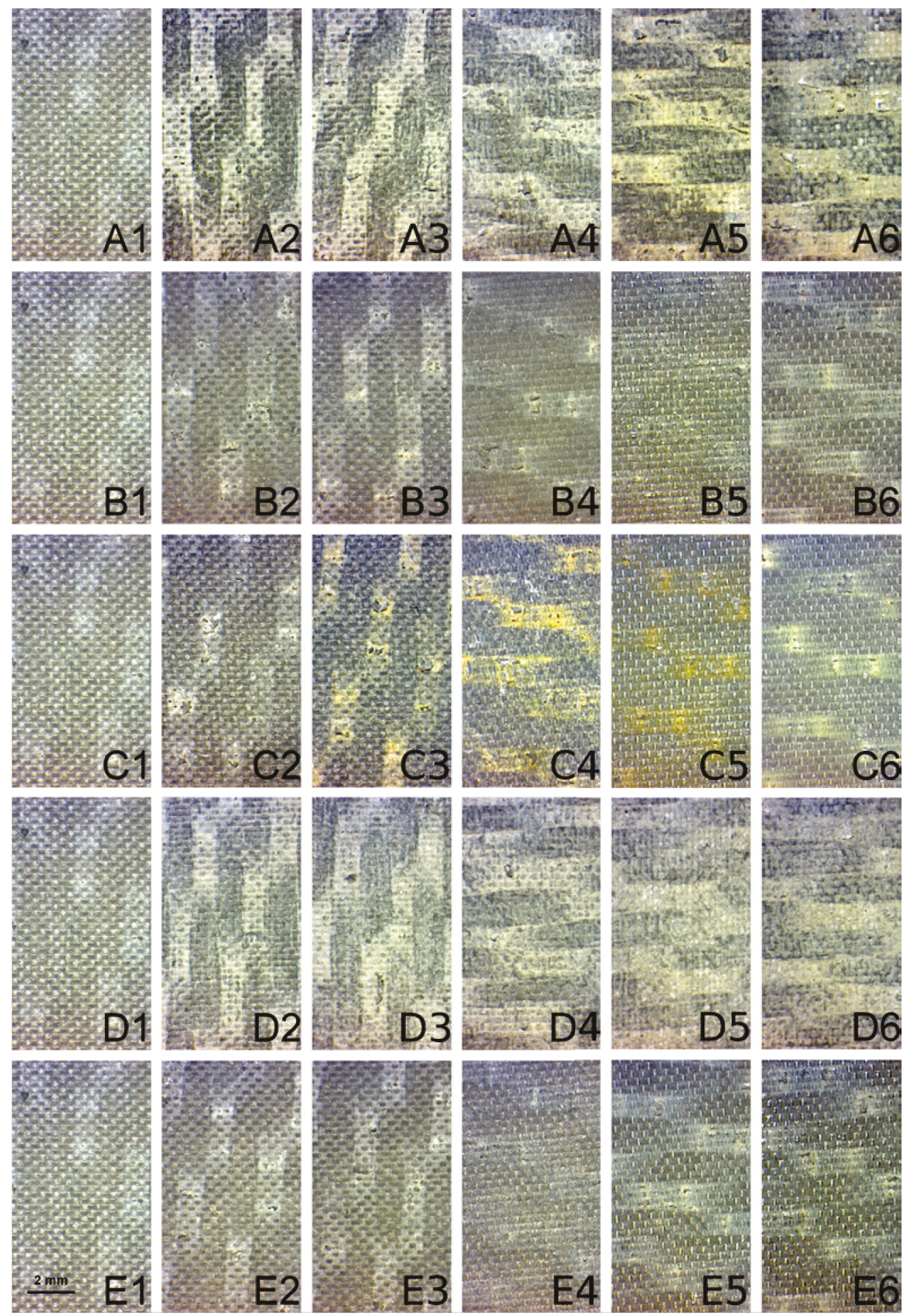

Figure D.2: Cross comparison of 3.5x magnification, bag-side flexural sample microscopy performed after 1) 0, 2) 24, 3) 168, 4) 720, 5) 1440 and 6) 2160 hours of exposure to A) deionized water, B) hydraulic fluid, C) ethylene glycol, D) ocean water and E) turbine oil. 

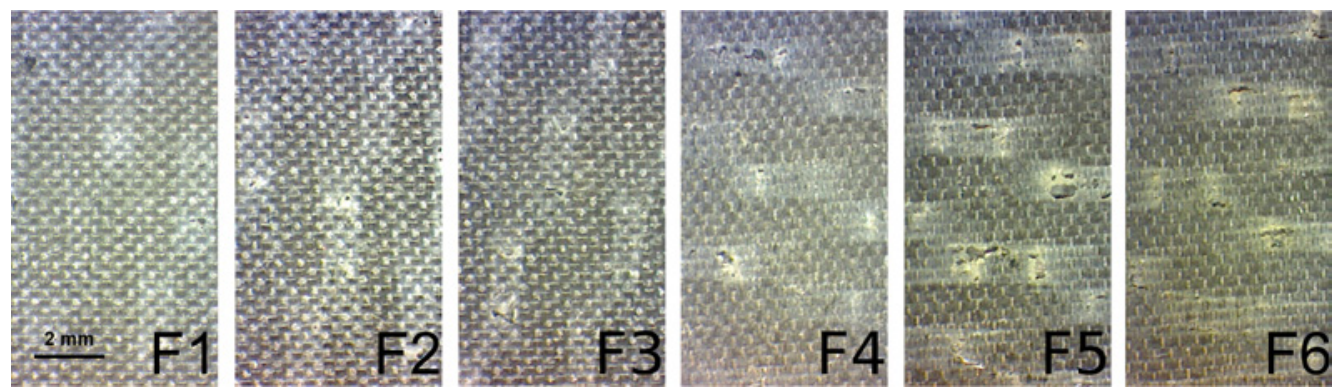

Figure D.3: Comparison of 3.5x magnification, bag-side flexural sample microscopy performed after 1) 0, 2) 24, 3) 168, 4) 720, 5) 1440 and 6) 2160 hours of exposure to air (control). 
Table D.1: SBS sample groups exposed to water conditioning, comparing dry-time, mass change and residual strength.

\begin{tabular}{|c|c|c|c|c|c|c|c|}
\hline $\begin{array}{l}\text { Sample } \\
\text { ID }\end{array}$ & $\begin{array}{c}\text { Conditioning } \\
\text { Fluid }\end{array}$ & $\begin{array}{c}\text { Intended Duration } \\
\text { (Days) }\end{array}$ & $\begin{array}{c}\text { Duration Dry } \\
\text { (Days) }\end{array}$ & $\begin{array}{c}\text { Mass } \\
\text { Change }\end{array}$ & $\begin{array}{c}\text { Mass Standard } \\
\text { Deviation }\end{array}$ & $\begin{array}{l}\text { Residual } \\
\text { Strength }\end{array}$ & $\begin{array}{c}\text { Strength Standard } \\
\text { Deviation }\end{array}$ \\
\hline SBS_D02 & & & & $0.762 \%$ & & $98.236 \%$ & \\
\hline SBS_D01 & DI Water & 1 & - & $1.050 \%$ & $0.173 \%$ & $95.210 \%$ & $1.556 \%$ \\
\hline SBS_B12 & & & & $0.739 \%$ & & $97.351 \%$ & \\
\hline SBS_B17 & & & & $1.531 \%$ & & $73.637 \%$ & \\
\hline SBS_E11 & DI Water & 7 & - & - & $0.043 \%$ & $75.078 \%$ & $2.734 \%$ \\
\hline SBS_D04 & & & & $1.591 \%$ & & $69.790 \%$ & \\
\hline SBS_D06 & & & & $1.737 \%$ & & $44.415 \%$ & \\
\hline SBS_B07 & DI Water & 14 & - & $1.556 \%$ & $0.161 \%$ & $46.255 \%$ & $0.949 \%$ \\
\hline SBS_C03 & & & & $1.877 \%$ & & $44.934 \%$ & \\
\hline SBS_E12 & & & & $0.667 \%$ & & $48.560 \%$ & \\
\hline SBS_E26 & DI Water & 35 & - & $0.683 \%$ & $0.041 \%$ & $49.692 \%$ & $0.629 \%$ \\
\hline SBS_E03 & & & & $0.606 \%$ & & $48.650 \%$ & \\
\hline SBS_H13 & & & 6 & $0.494 \%$ & & $47.585 \%$ & \\
\hline SBS_G09 & DI Water & 63 & 6 & $0.236 \%$ & $0.131 \%$ & $47.716 \%$ & $0.245 \%$ \\
\hline SBS_G16 & & & 6 & $0.406 \%$ & & $47.242 \%$ & \\
\hline SBS_H19 & & & & $0.242 \%$ & & & \\
\hline SBS_F14 & DI Water & 63 & - & - & $0.139 \%$ & - & - \\
\hline SBS_F25 & & & & $0.438 \%$ & & & \\
\hline SBS_A08 & & & & $1.115 \%$ & & $96.301 \%$ & \\
\hline SBS_D07 & SO Water & 1 & - & $1.405 \%$ & $0.188 \%$ & $88.297 \%$ & $4.962 \%$ \\
\hline SBS_B03 & & & & $1.053 \%$ & & $97.379 \%$ & \\
\hline SBS_C01 & & & & $1.829 \%$ & & $75.607 \%$ & \\
\hline SBS_D05 & SO Water & 7 & - & $2.969 \%$ & $0.658 \%$ & $70.790 \%$ & $2.923 \%$ \\
\hline SBS_A18 & & & & $2.969 \%$ & & $76.067 \%$ & \\
\hline SBS_B09 & & & & $2.842 \%$ & & $41.145 \%$ & \\
\hline SBS_E27 & SO Water & 14 & - & $2.833 \%$ & $0.011 \%$ & $38.965 \%$ & $1.322 \%$ \\
\hline SBS_E23 & & & & $2.854 \%$ & & $38.758 \%$ & \\
\hline SBS_E22 & & & & $2.349 \%$ & & $46.615 \%$ & \\
\hline SBS_E02 & SO Water & 35 & - & $2.547 \%$ & $0.203 \%$ & $46.525 \%$ & $0.388 \%$ \\
\hline SBS_E20 & & & & $2.140 \%$ & & $47.237 \%$ & \\
\hline SBS_F16 & & & 6 & $2.329 \%$ & & $44.707 \%$ & \\
\hline SBS_F01 & SO Water & 63 & 6 & $2.101 \%$ & $0.189 \%$ & $45.955 \%$ & $0.830 \%$ \\
\hline SBS_F13 & & & 6 & $1.953 \%$ & & $46.280 \%$ & \\
\hline SBS_G25 & & & & $1.332 \%$ & & & \\
\hline SBS_F19 & SO Water & 63 & - & $1.952 \%$ & $0.351 \%$ & - & - \\
\hline SBS_H16 & & & & $1.926 \%$ & & & \\
\hline
\end{tabular}




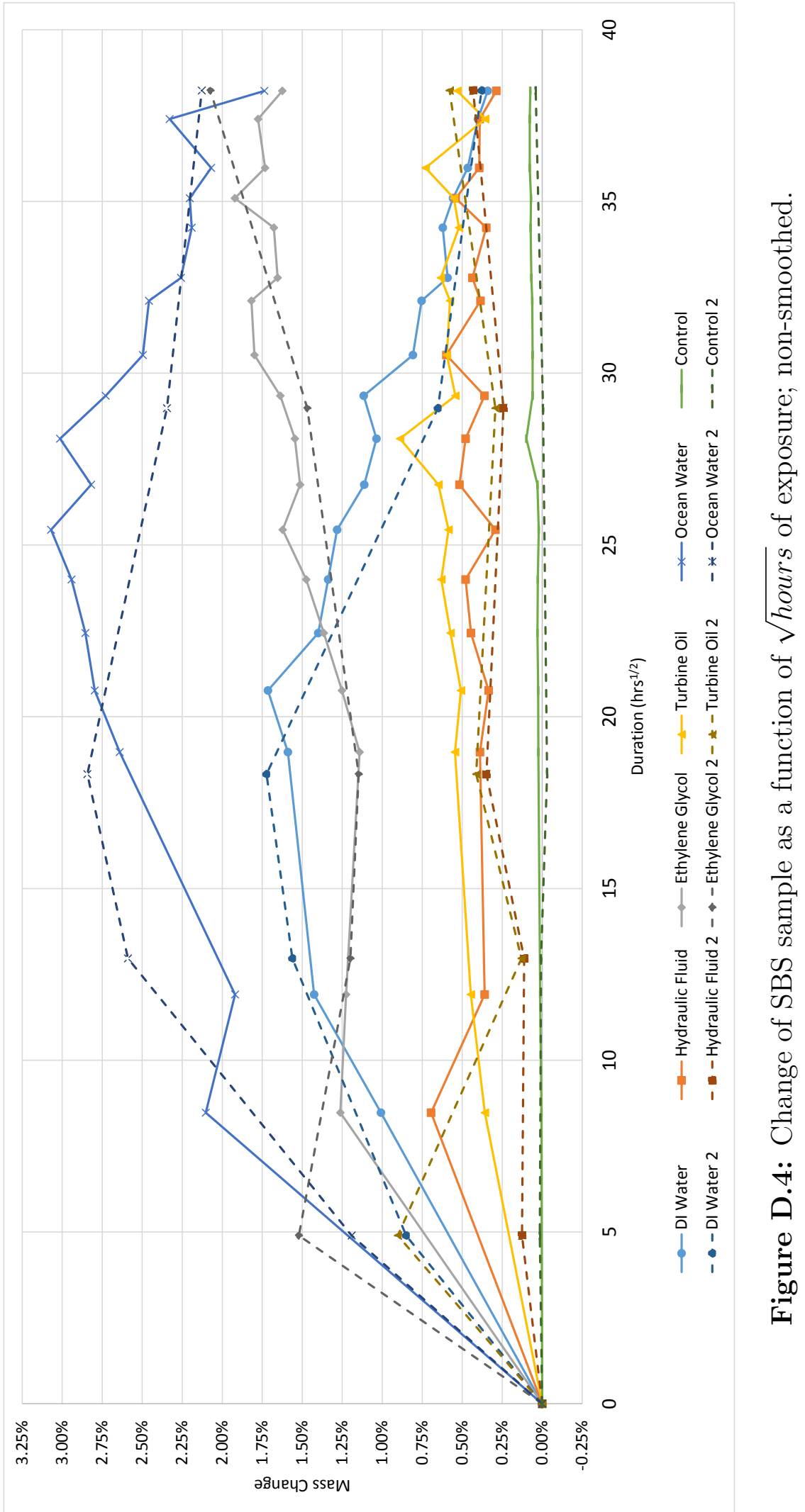


Table D.2: Two-factor ANOVA summary for SBS testing.

\begin{tabular}{cccccc}
\hline $\begin{array}{c}\text { Source of } \\
\text { Variation }\end{array}$ & $\begin{array}{c}\text { Sum of } \\
\text { Squares }\end{array}$ & $\begin{array}{c}\text { Degrees of } \\
\text { Freedom }\end{array}$ & $\begin{array}{c}\text { Mean } \\
\text { Square }\end{array}$ & $\boldsymbol{F}_{\mathbf{0}}$ & P-value \\
\hline Chemical & 7280.6678 & 5 & 1456.1336 & 173.64138 & $2.464 \mathrm{E}-34$ \\
Time & 958.14768 & 4 & 239.53692 & 28.56436 & $2.656 \mathrm{E}-13$ \\
Interaction & 2394.4144 & 20 & 119.72072 & 14.276487 & $6.277 \mathrm{E}-16$ \\
Error & 503.15202 & 60 & 8.385867 & & \\
Total & 11136.382 & 89 & & & \\
\hline
\end{tabular}

Table D.3: Summary of Dunnett and Tukey post hoc statistical analysis of SBS data; significant differences in means highlighted in bold.

\begin{tabular}{cccccc}
\hline Duration (Days) & $\mathbf{1}$ & $\mathbf{7}$ & $\mathbf{1 4}$ & $\mathbf{3 5}$ & $\mathbf{6 3}$ \\
\hline \multicolumn{5}{c}{ Dunnett's Test } \\
\hline$D_{\alpha}$ & 6.573 & 6.928 & 7.334 & 7.544 & 7.799 \\
\hline DI Water & 1.7126 & $\mathbf{1 5 . 1 6 4 5}$ & $\mathbf{2 5 . 2 3 2 9}$ & $\mathbf{2 8 . 4 8 8 1}$ & $\mathbf{2 9 . 2 9 9 2}$ \\
Hydraulic Fluid & 3.9119 & 0.2955 & 1.6165 & 0.2504 & 0.5680 \\
Ethylene Glycol & 0.9330 & 2.4852 & 0.7005 & 3.6410 & 1.2484 \\
Turbine Oil & 0.4019 & 1.3284 & 1.9934 & 2.7911 & 1.8274 \\
Ocean Water & 3.3536 & $\mathbf{1 4 . 4 2 7 7}$ & $\mathbf{2 2 . 1 1 8 5}$ & $\mathbf{2 9 . 7 0 2 1}$ & $\mathbf{3 0 . 3 4 1 6}$ \\
Control & 2.4783 & 1.3928 & 0.1348 & 1.5352 & 3.3769 \\
\hline$q_{\alpha}$ & 7.5770 & 7.9862 & 8.4542 & 8.6961 & 8.9906 \\
$\left|y_{D I}-y_{S O}\right|$ & 1.6411 & 0.7367 & 3.1145 & 1.2140 & 1.0424 \\
\hline
\end{tabular}




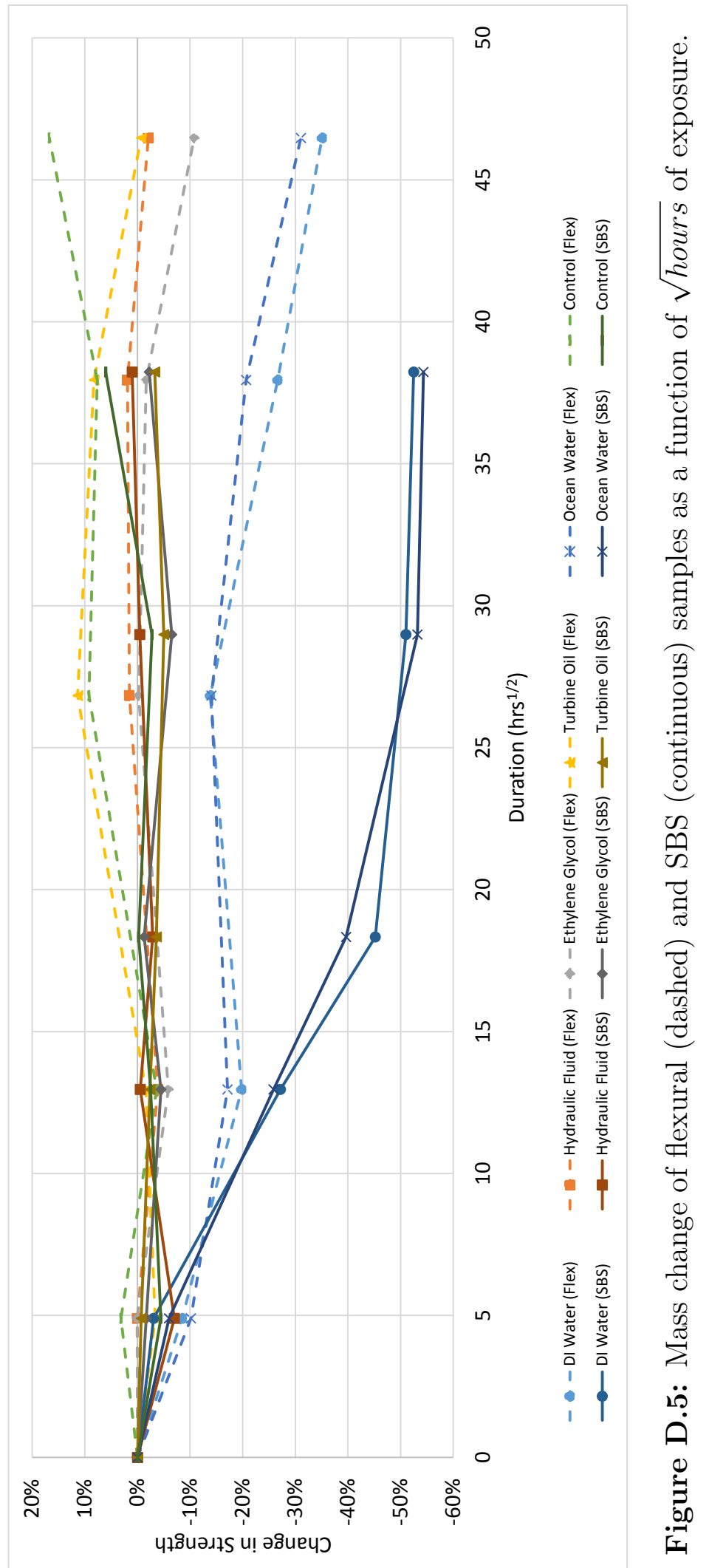




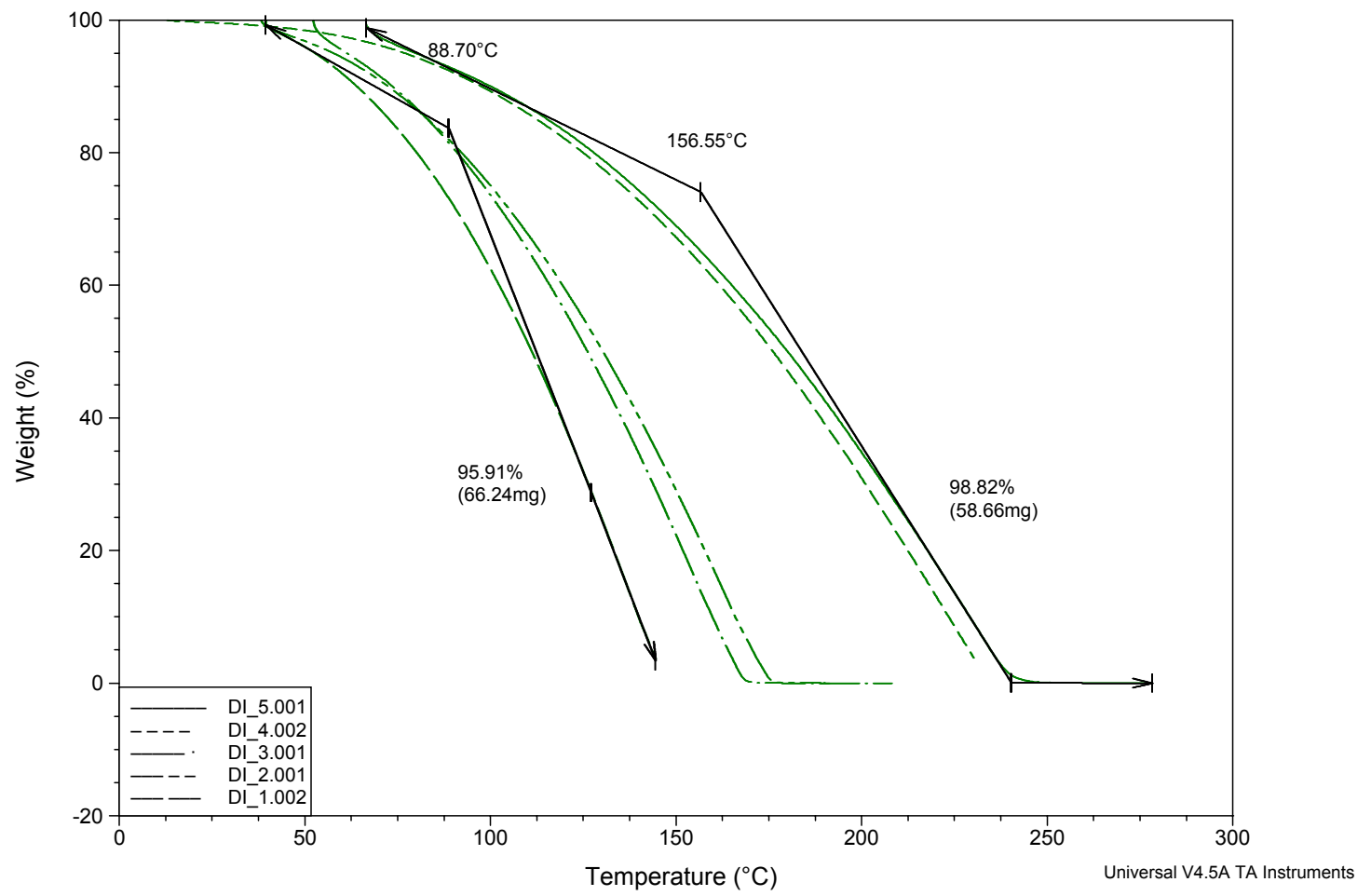

Figure D.6: Baseline TGA of DI water evaporation, heated at rates of $10^{\circ} \mathrm{C} / \mathrm{min}$, $20^{\circ} \mathrm{C} / \mathrm{min}, 30^{\circ} \mathrm{C} / \mathrm{min}, 40^{\circ} \mathrm{C} / \mathrm{min}$ and $50^{\circ} \mathrm{C} / \mathrm{min}$. 


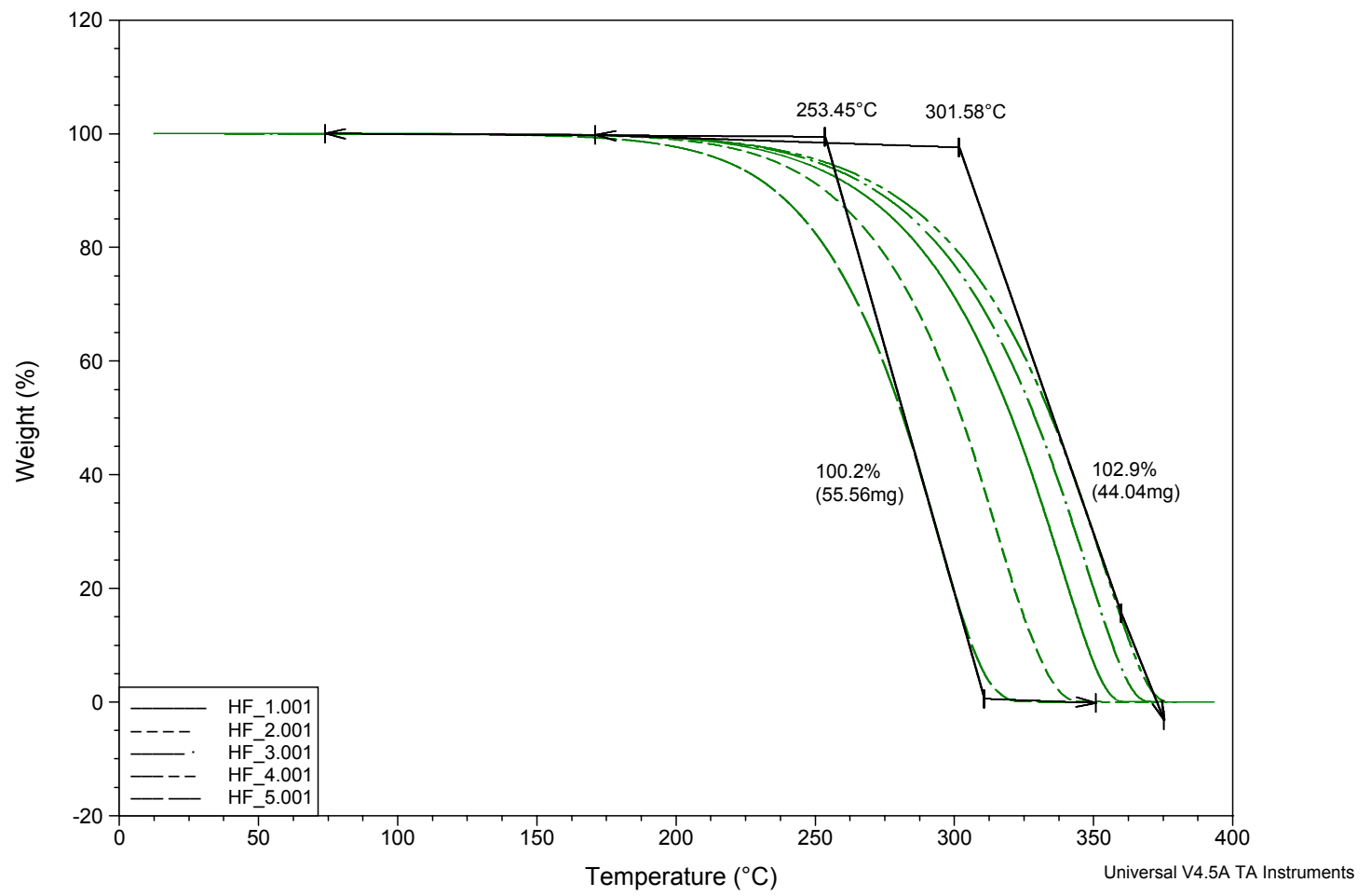

Figure D.7: Baseline TGA of hydraulic fluid evaporation, heated at rates of $10^{\circ} \mathrm{C} / \mathrm{min}, 20^{\circ} \mathrm{C} / \mathrm{min}, 30^{\circ} \mathrm{C} / \mathrm{min}, 40^{\circ} \mathrm{C} / \mathrm{min}$ and $50^{\circ} \mathrm{C} / \mathrm{min}$. 


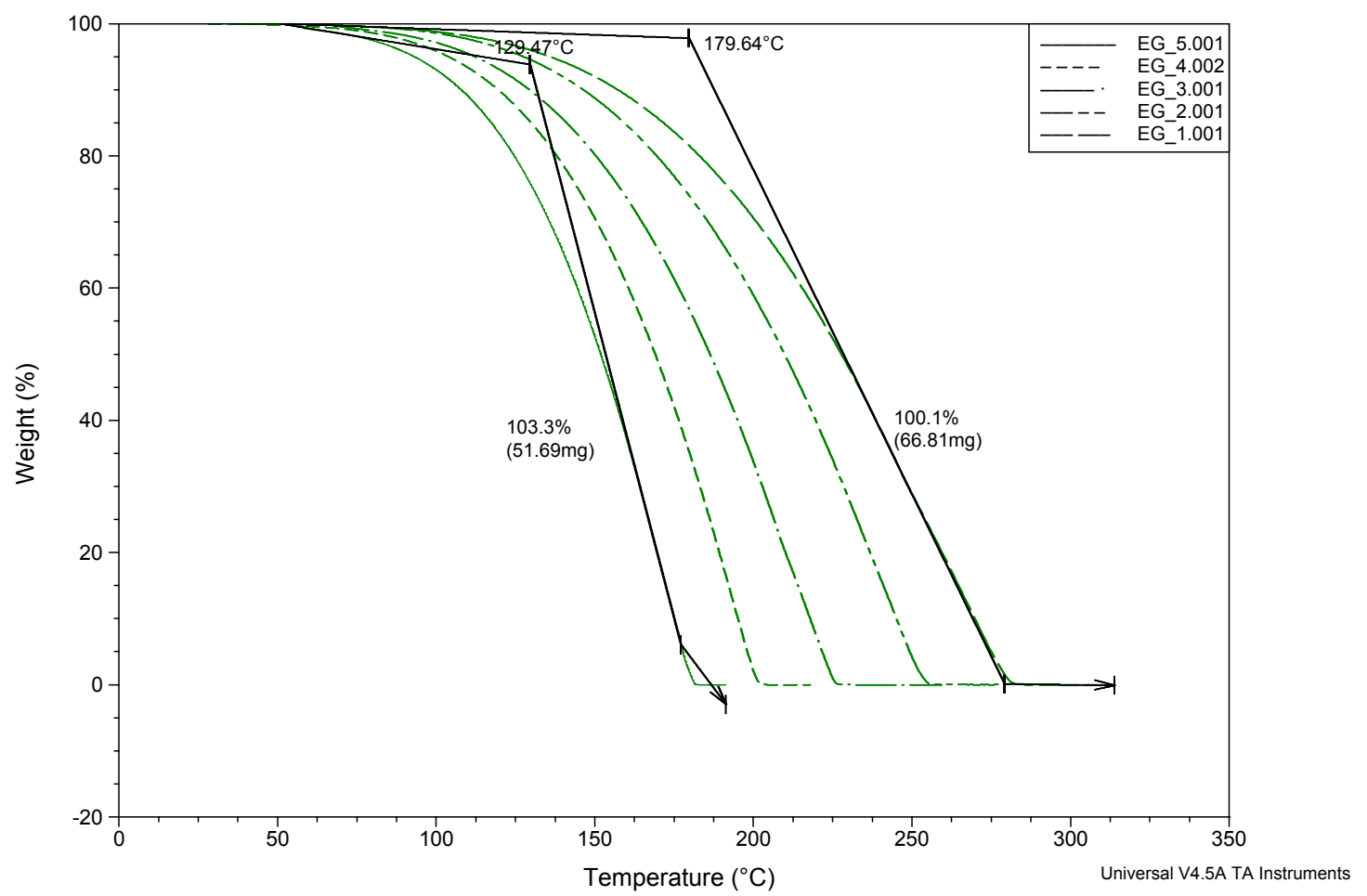

Figure D.8: Baseline TGA of ethylene glycol evaporation, heated at rates of $10^{\circ} \mathrm{C} / \mathrm{min}, 20^{\circ} \mathrm{C} / \mathrm{min}, 30^{\circ} \mathrm{C} / \mathrm{min}, 40^{\circ} \mathrm{C} / \mathrm{min}$ and $50^{\circ} \mathrm{C} / \mathrm{min}$. 


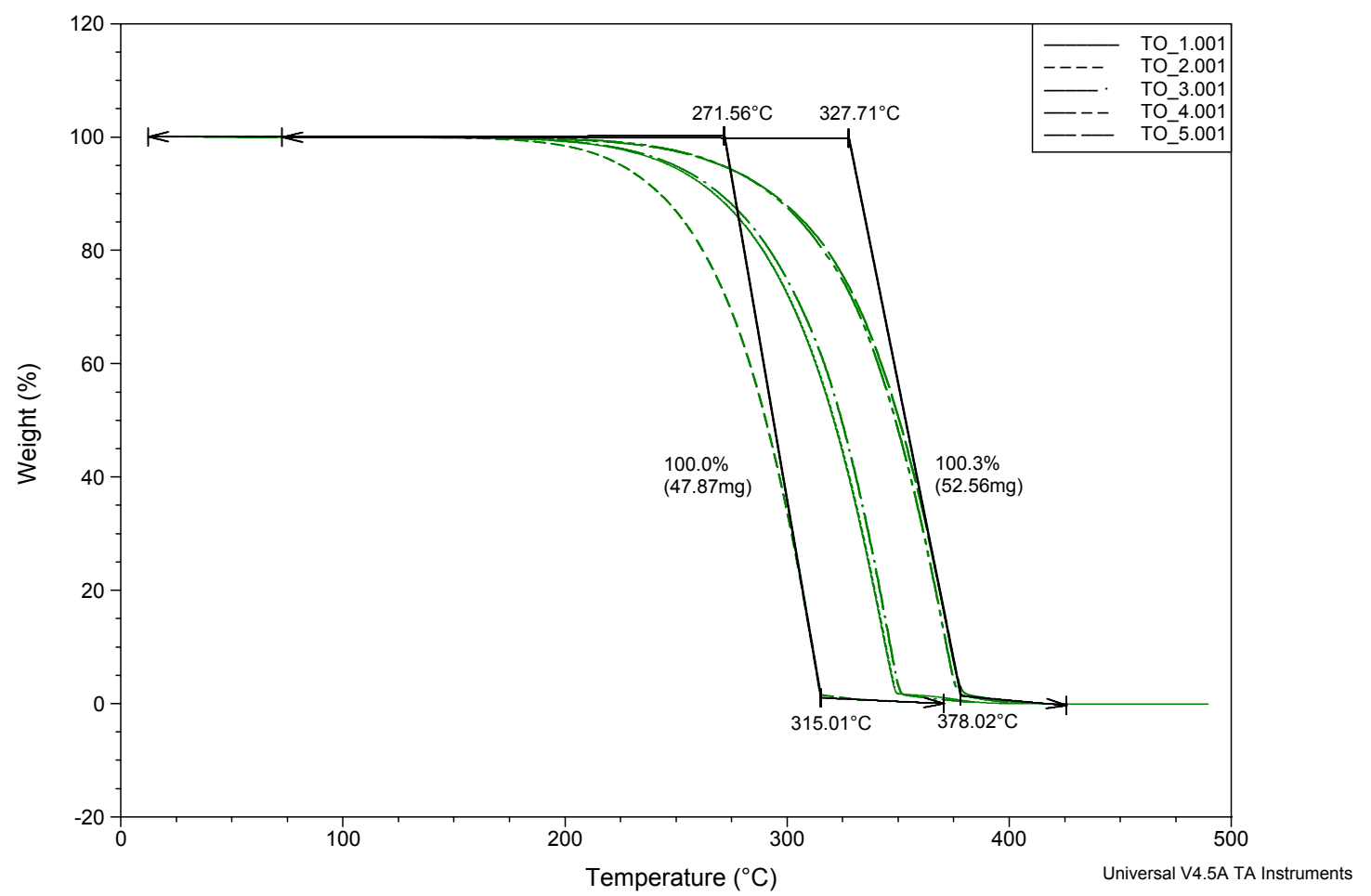

Figure D.9: Baseline TGA of turbine oil evaporation, heated at rates of $10^{\circ} \mathrm{C} / \mathrm{min}$, $20^{\circ} \mathrm{C} / \mathrm{min}, 30^{\circ} \mathrm{C} / \mathrm{min}, 40^{\circ} \mathrm{C} / \mathrm{min}$ and $50^{\circ} \mathrm{C} / \mathrm{min}$. 


\section{Appendix E}

\section{Micro-computed Tomography Code}

Presented in this appendix is the code developed and implemented in order to analyze the obtained micro-computed tomography image sets for percentage void content and void distribution. Code was written in Python programming language using the Microsoft Visual Studio 2017 integrated development environment.

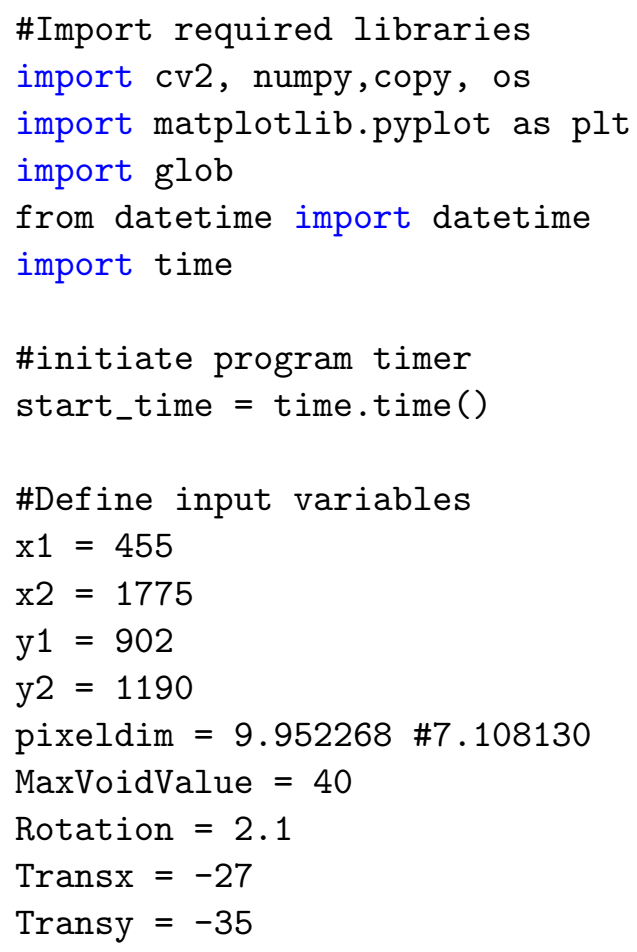




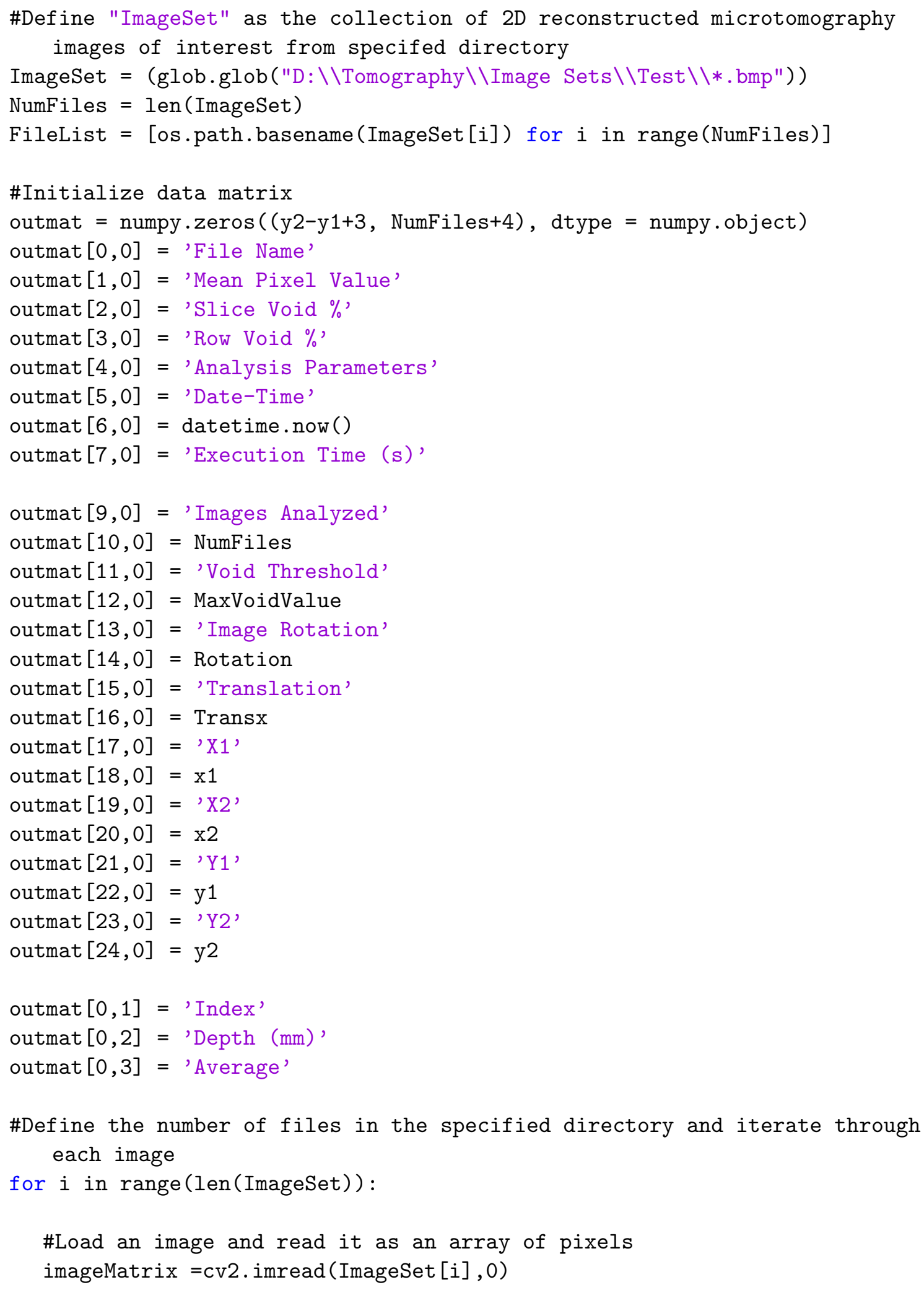




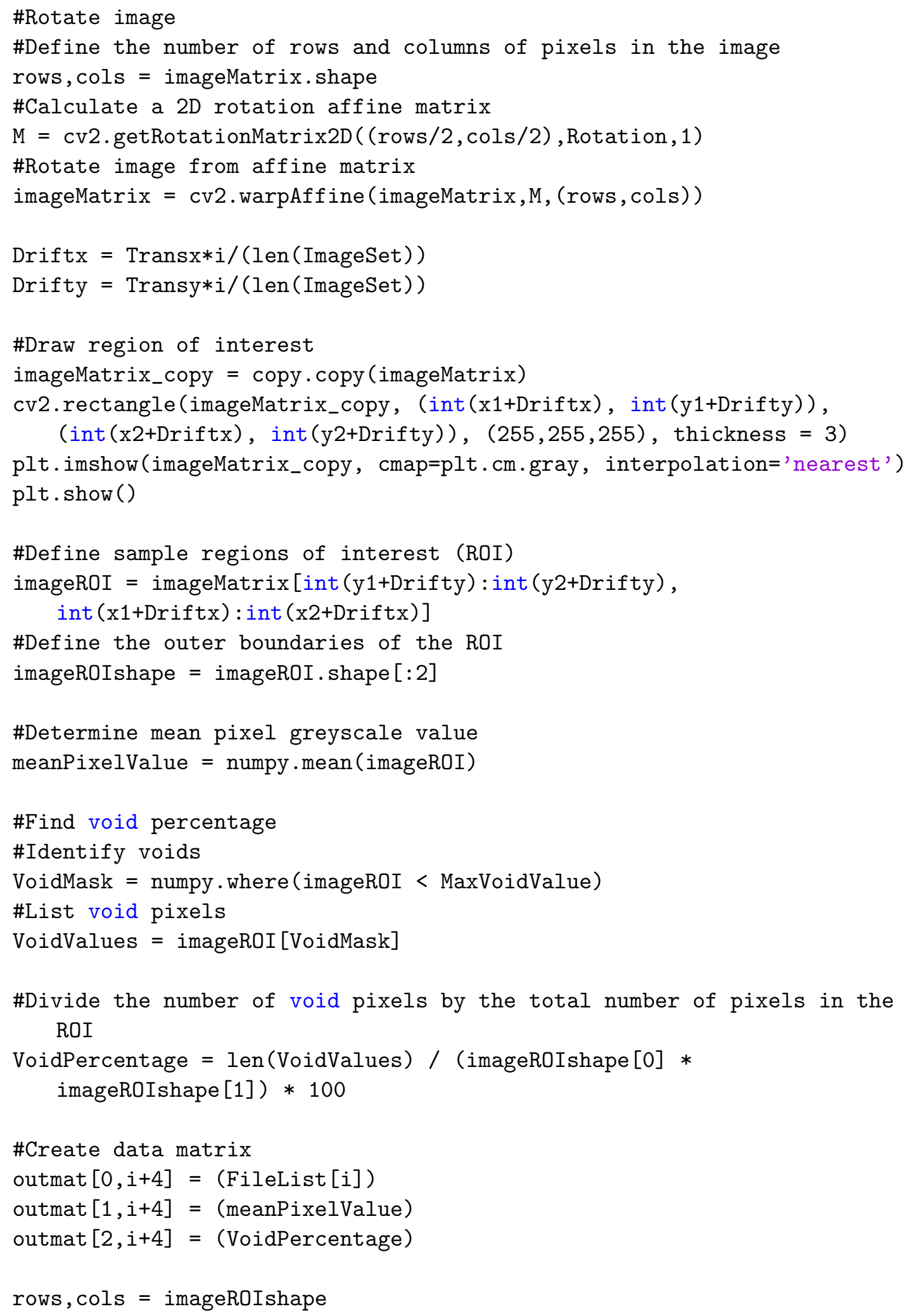




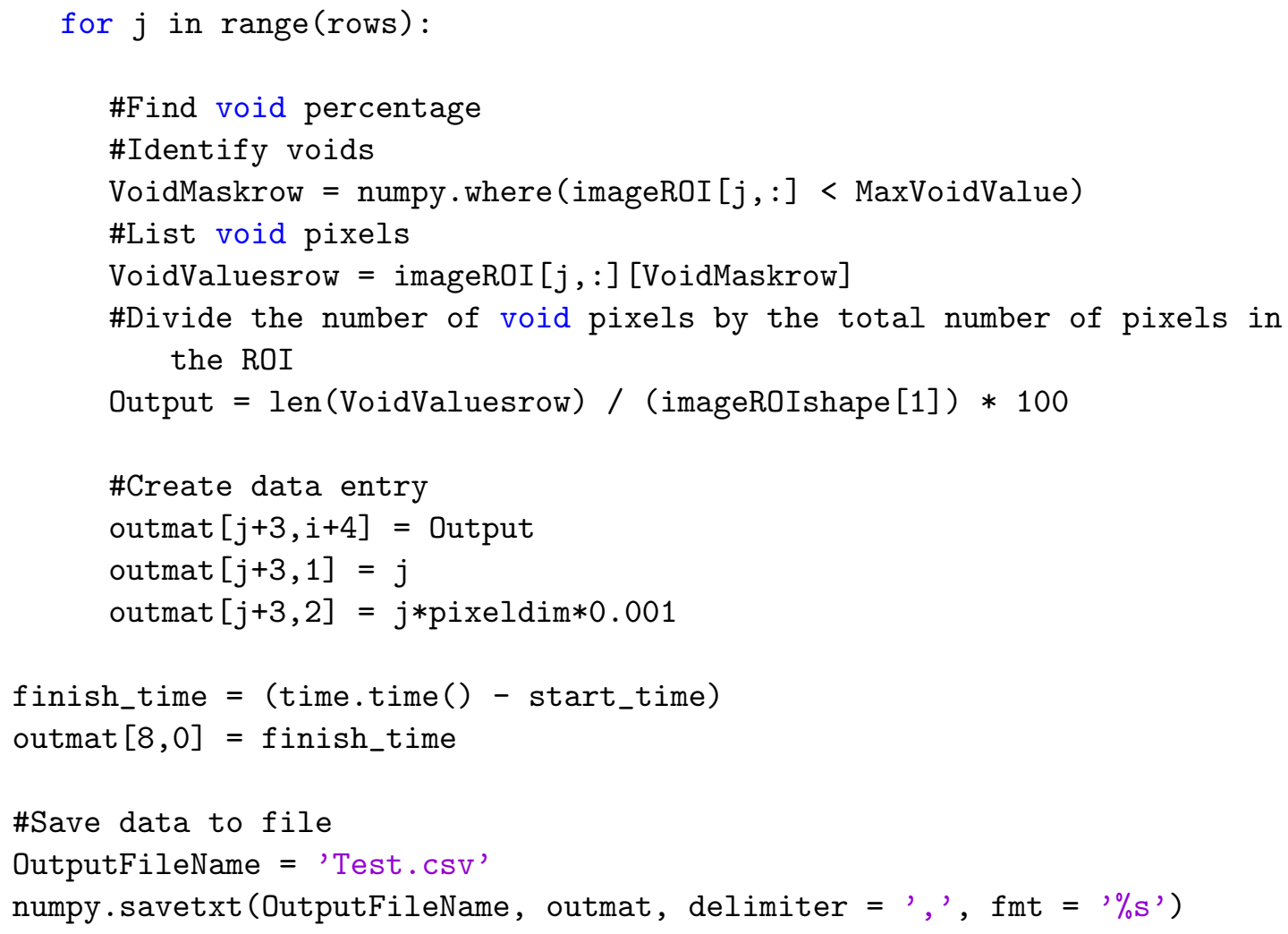

